\title{
Seismic anisotropy and velocity structure in the North Island, New Zealand
}

\author{
by \\ Hubert Jerzy Zal
}

\author{
A thesis \\ submitted to Victoria University of Wellington \\ in fulfillment of the \\ requirements for the degree of \\ Doctor of Philosophy \\ in Geophysics
}

School of Geography, Environment and Earth Science

Victoria University of Wellington

Wellington, New Zealand 



\section{Abstract}

This thesis investigates crustal and upper mantle seismic anisotropy, via shear wave splitting (SWS) analysis, across the Hikurangi subduction zone of the North Island, New Zealand. Seismic anisotropy is defined as a directionally dependent elastic response of seismic waves to an anisotropic material. Seismic anisotropy in the Earth can arise from a number of causes, including stress-induced alignment of cracks, alignment of anisotropic crystals, structures (e.g., fault fabric), and lithology. SWS is a powerful technique used to examine anisotropy beneath the surface of the Earth and is one of the few methods available to measure stress in the crust and lithosphere as well as strain and flow in the mantle. The North Island of New Zealand provides an excellent laboratory to study plate boundary deformation processes. The abundance of seismic data for North Island makes it possible to perform SWS analyses across the Hikurangi subduction zone. Several SWS studies have been conducted in North Island, but few have covered the region in high detail, leaving large swathes of North Island unstudied. Here, high-resolution SWS analysis is performed in three distinct areas covered by seismic array experiments. Of these three areas, one is offshore and two are on-land, and each presents a distinct geodynamic context and associated challenges.

The first study investigated an area offshore the east coast of North Island, which is a region of frequent slow slip. This study builds on the success of the Hikurangi Ocean Bottom Investigation of Tremor and Slow Slip (HOBITSS) experiment, whose array of ocean bottom instruments captured, for the first time, a large shallow slow slip event (SSE). Understanding the physical processes occurring during SSEs is particularly important from an earthquake hazard perspective, as SSEs may influence the timing of nearby large earthquakes, or alternatively be triggered by earthquakes. The data collected by the HOBITSS instruments are analyzed to extend crustal splitting results offshore and to examine temporal and spatial varia- 
tions in crustal anisotropy during an SSE. The complementary sensitivity of $V_{P} / V_{S}$ and SWS measurements to cracks in the upper crust allowed us to evaluate the anisotropy before, during and after the September-October 2014 SSE. Temporal variations in $V_{P} / V_{S}$ and delay time are observed during the SSE and are consistent with fluid pressurization below a permeability barrier and movement of fluids during the build-up to and rupture of a slow-slip patch. This study demonstrates that SWS and $V_{P} / V_{S}$ are effective tools for investigating stress changes and fluid migration during SSEs.

The second study focused on the central North Island, which is covered by an array of permanent GeoNet stations. I produced one of the largest datasets of crustal anisotropy measurements for this region. Using this dataset, we investigated the relationship between seismic anisotropy and the stress state by examining 42,423 high-quality SWS measurements across 24 GeoNet land-based seismic stations. The initial aim was to search for temporal changes in the state of the crust during one of the longest and deepest SSEs recorded in New Zealand; however, we did not find any significant temporal variations in our SWS results. We compared our SWS fast polarization azimuths to stress orientations derived from continuous campaign GPS (Global Positioning System) and gravitational stress calculations, as well as orientations of active faults. The spatial averaging of SWS fast polarizations azimuths showed significant spatial variations across central North Island. Comparisons with other measurements helped to reveal dominant influences. The fast azimuths at many stations across the North Island Dextral Fault Belt (NIDFB) were consistent with the regional NE-SW fault orientations, suggesting a strong structural control on anisotropy. Two regions showed clear deviations from the structural trend and were more similar to the regional maximum compressional stress $S_{H} \max$. These included fast azimuths within the Wanganui Basin, as well as in a small area along the NIDFB. Additionally, fast azimuths around Mt. Ruapehu showed complex variations and resemblance to both $S_{H} \max$ and fault orientations, suggesting a combination of both stress-induced and structural control. With knowledge of the local stress field, as well as structural elements in this region we were able to determine whether crustal anisotropy is caused by stress, structure, or a combination of both mechanisms.

The third study investigated the relationship between seismic anisotropy and mantle deformation across a deep boundary in the mantle, marked by the Taranaki- 
Ruapehu Line (TRL), in the back-arc region of western North Island. The TRL marks the boundary between a deeper crust ( $\sim 32 \mathrm{~km}$ thick) to the south and a shallower crust ( $\sim 25 \mathrm{~km}$ thick) in the north, interpreted as an abrupt step in the Moho. Numerous geophysical studies have examined changes across the TRL, such as in electrical conductivity, gravity, seismic attenuation, and crustal thickness, but few studies have provided constraints on the upper mantle structure from seismic anisotropy. We investigated upper mantle anisotropic properties across the TRL by analyzing SWS measurements from teleseismic earthquakes recorded on a temporary seismic array, the Ruapehu And Taranaki Teleseismic Imaging Line (RATTIL) network between 2012-2014. SWS measurements revealed a strong NESW $\left(42^{\circ}\right)$ oriented anisotropy across the TRL. The similarity of our fast azimuths to previous studies in eastern North Island where there is no wedge under the stations suggests that similar fast azimuths are found in both the mantle wedge and subslab mantle. The dominant trench-parallel orientation of our fast azimuth measurements are likely due to the NE-SW lattice-preferred orientation of olivine in the mantle wedge due to shear deformation associated with oblique convergence and trench-parallel mantle flow. Previous studies have observed apparent isotropy, dominantly west of the central volcanic region, and have suggested that isotropy may extend as far south as the TRL. Our results show that this region is anisotropic and we suggest that the boundary between apparent isotropy to the north and anisotropy in the south is located north of the RATTIL network $\left(-38.75^{\circ}\right)$.

Overall, this work represents an extensive analysis of seismic anisotropy across North Island. The results provide a detailed view of the lateral variations of seismic anisotropy across North Island and they inform us about the underlying anisotropic sources. Moreover, these results shed new light on the temporal and spatial dynamics of a slow slip event and reveal its mechanics. The gained insights will be useful for further development of SWS and future anisotropy analyses. 


\section{Acknowledgments}

There are many people who helped me along my PhD journey. Firstly, I would like to thank my primary supervisor Dr. Martha Savage, for her unwavering support and guidance. Thank you for always leaving your door open and for your scientific advice on seismic anisotropy - and beyond. Your meritorious feedback and encouragement helped me overcome many challenges. I would also like to thank my secondary supervisor Dr. Katrina Jacobs, for all the time and invaluable feedback that you gave me during my research. I am proud to be your first PhD student.

I would like to acknowledge everyone involved in the HOBITSS project. Thank you to Dr. Anne Sheehan, Jefferson Yarce, Dr. Laura Wallace, and Dr. Kimi Mochizuki for providing data and feedback. I am grateful to Dr. Kimi Mochizuki for the opportunity to intern at the Earthquake Research Institute at the University of Tokyo, Japan and for his warm hospitality and fun trips. Thank you, Stefan Mroczek, Kenny Graham, Dr. Erin Todd, Dr. Jenny Nakai, Yuriko Iwasaki, Dr. Susan Schwartz, Dr. Spahr Webb, Dr. Stuart Henrys, Dr. Emily Warren-Smith and Dr. Carolyn Boulton for your thoughtful discussions and suggestions and your invaluable input to my first publication.

Thank you, Dr. Simon Lamb and Hamish Hirschberg, for your expert advice with Chapter 4 and Dr. Tim Stern and Dr. Jesse-Lee Dimech who provided me with the data and feedback for Chapter 5. Thank you for always making yourselves available to answer burning geophysics related questions.

I experienced some of the most memorable and happiest events during my $\mathrm{PhD}$. The happiest of which was when I met my future wife, to whom I dedicate this thesis. Olya, you are not only my love and muse, but also my closest friend and biking partner. There is nothing like coming home to you after long hours in the 
office.

Thank you to Dr. John Townend and Dr. Rupert Sutherland for your guidance and advice. I would also like to thank my past and present office mates, Katie Woods, Lucie Yancoo, Weiwei Wang, Konrad Weaver, Kenny Graham, Lukas Eling, Pegah Faegh Lashgary, Bryant Chow, Konstantinos Michailos, Dominic Evanzia, Finn Illsley-Kemp, Calum Chamberlain, Emily Warren-Smith, Steven Sewell, Chet Hopp, Stefan Mroczek, Hamish Hirschberg, Thomas Wilson, Laura-May Baratin, Francesco Civilini, Rachel Heckels, Lucek Sajkowski, Cecile Massiot, Jesse-Lee Dimech and Holly Godfrey for the fun times and a great work environment. Thank you, Aleksandr Beliaev and Adrian Benson for your expertise in the field and technical support. Thank you, Dr. Cynthia Ebinger for your continual guidance and for getting me in touch with Martha Savage, which ultimately set me on this path.

Finally, a special and heartfelt thanks go to my parents, Anna and Tomasz, and my sister Paulina, for always believing in me and helping me to pursue science. 


\section{Contents}

$\begin{array}{lll}\text { Abstract } & \text { iii }\end{array}$

Acknowledgments vii

$\begin{array}{ll}\text { Contents } & \text { ix }\end{array}$

List of Figures $\ldots \ldots \ldots \ldots \ldots \ldots \ldots \ldots \ldots$

List of Tables . . . . . . . . . . . . . . . . . . xix

1 Introduction and background 1

1.1 Introduction . . . . . . . . . . . . . . . . . . 1

1.2 Thesis content ...................... 2

1.2.1 Statement of contributions . . . . . . . . . . . 5

1.3 North Island, New Zealand . . . . . . . . . . . . . . . . . . 5

1.3.1 Tectonic setting of North Island, New Zealand . . . . . . . . 5

1.3.2 The Hikurangi subduction zone . . . . . . . . . . . . . 9

1.3.3 Seismicity of North Island, New Zealand . . . . . . . . . . . 14

1.4 Seismic anisotropy $\ldots \ldots \ldots \ldots$. . . . . . . . . . . . . 14

1.4.1 Theoretical background . . . . . . . . . . . 16

1.4 .2 Origins of anisotropy . . . . . . . . . . . 22

1.4.3 Previous shear wave splitting studies . . . . . . . . . 27 
2 Data and methodology 33

2.1 Data acquisition . . . . . . . . . . . . . . . 33

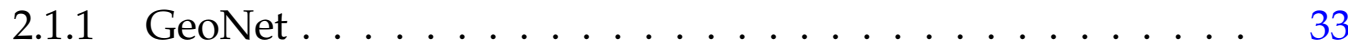

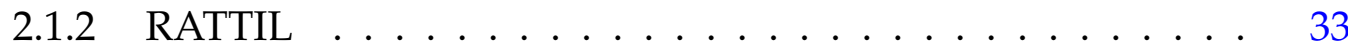

2.1 .3 HOBITSS ........................ 34

2.2 HOBITSS ocean bottom seismometer orientations . . . . . . . . 35

2.2.1 P-wave orientation . . . . . . . . . . . . 35

2.2.2 Surface wave orientation $\ldots \ldots . \ldots 37$

2.2.3 Removal of poor seismic data . . . . . . . . . . . . . . 39

2.2.4 Horizontal orientation results . . . . . . . . . . . . . . 39

2.3 Shear wave splitting techniques . . . . . . . . . . . . . . . . 41

2.3.1 MFAST: Automatic Shear Wave Splitting . . . . . . . . . . . 41

2.3.2 SplitRacer: Shear Wave Splitting . . . . . . . . . . . . . . . 45

3 Temporal and spatial variations in seismic anisotropy and $V_{P} / V_{S}$ ratios in a region of slow slip 49

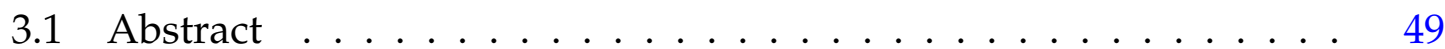

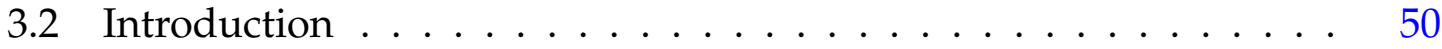

3.2.1 Hikurangi Subduction Zone and the September-October 2014

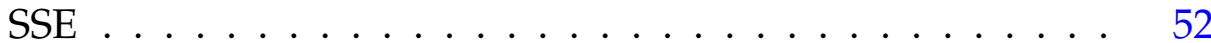

3.2 .2 Previous results . . . . . . . . . . . . . 54

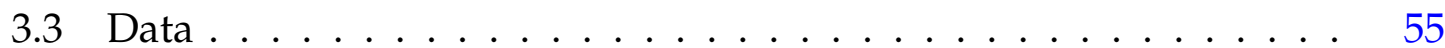

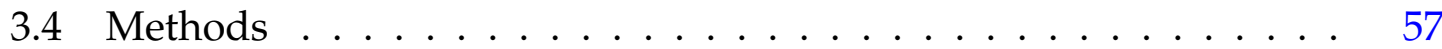

3.4.1 Automatic Shear Wave Splitting (MFAST) . . . . . . . . . . . 57

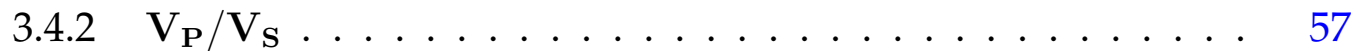

3.4.3 Moving averages and 95\% confidence intervals . . . . . . 58 
3.4 .4 Spatial averaging $\ldots \ldots \ldots \ldots \ldots$

3.5 Results . . . . . . . . . . . . . . . . . 59

3.5.1 Fast azimuths and spatial averaging . . . . . . . . 59

3.5.2 Temporal changes in fast azimuths, delay time and $V_{P} / V_{S}$ measurements . . . . . . . . . . . . . . . 60

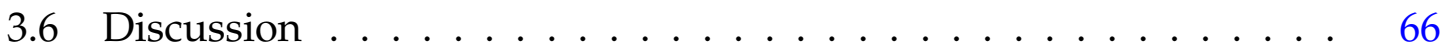

3.7 Conclusions . . . . . . . . . . . . . . . . 72

3.8 Acknowledgments . . . . . . . . . . . . . . . 74

4 Spatial variations in seismic anisotropy in central North Island, New Zealand 75

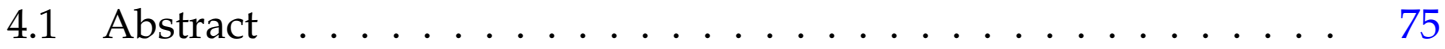

4.2 Introduction . . . . . . . . . . . . . . . 76

4.2 .1 Tectonic setting . . . . . . . . . . . 76

4.2 .2 Slow slip events . . . . . . . . . . . . . 78

4.2 .3 Crustal anisotropy $\ldots \ldots \ldots \ldots 78$

4.2.4 Identifying principal stress orientations in the crust . . . . 81

4.3 Data and methods . . . . . . . . . . . . . . . . 85

4.3.1 Local phase arrival picking . . . . . . . . . . 85

4.3.2 Automatic Shear Wave Splitting (MFAST) . . . . . . . . . . . 86

$4.3 .3 \mathrm{~V}_{\mathbf{P}} / \mathrm{V}_{\mathbf{S}} \ldots \ldots \ldots \ldots \ldots$

4.3.4 Spatial averaging of fast azimuths . . . . . . . . . 87

4.3.5 Quantitative comparison of fast azimuth measurements . . 88

4.3.6 Spatial averaging of delay times . . . . . . . . . . . 99 90

4.3.7 Temporal analysis . . . . . . . . . . . . . . . 9 90

4.4 Results . . . . . . . . . . . . . . . . . . . . . 91 
4.4.1 Temporal analysis . . . . . . . . . . . . . . 91

4.4.2 Individual station averaged fast polarization azimuths . . . 93

4.4.3 Spatial averaging of fast polarization azimuths . . . . . . . 95

4.4.4 Depth extent of anisotropy . . . . . . . . . . . 95

4.4.5 Comparison of fast azimuths to stress models and fault orientations . . . . . . . . . . . . . . . . 98

4.4.6 Spatial averaging of delay times . . . . . . . . . . 103

4.5 Discussion ............................ 103

4.5.1 Fast polarization azimuths . . . . . . . . . . . 103

4.5 .2 Delay times . . . . . . . . . . . . . . 110

4.6 Conclusions . . . . . . . . . . . . . . . . . . 111

5 Upper mantle anisotropy across the Taranaki-Ruapehu Line in North Island, New Zealand 113

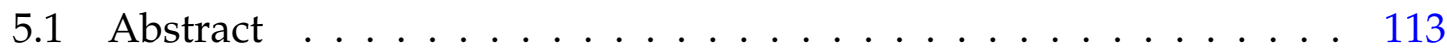

5.2 Introduction . . . . . . . . . . . . . . . 114

5.2 .1 Tectonic Setting . . . . . . . . . . . . . . . . . . . . . 114

5.2.2 Shear Wave Splitting . . . . . . . . . . . . . . . 117

5.2.3 Upper mantle anisotropy . . . . . . . . . . . . . . . . . 118

5.2.4 Previous upper mantle anisotropy studies . . . . . . . . . 119

5.3 Data and Methods . . . . . . . . . . . . . . . . . . 122

5.3.1 SplitRacer ...................... 122

5.4 Results .............................. 125

5.5 Discussion . . . . . . . . . . . . . . . . . 132

5.6 Conclusions . . . . . . . . . . . . . . . . . . . 136 
6 Conclusions

6.1 Implications for future research . . . . . . . . . . . . . . . . . . 144

Appendices

A Appendix A: Chapter 2 . . . . . . . . . . . . . . . 145

B Appendix B: Chapter $3 \ldots \ldots$. . . . . . . . . . . . 155

C Appendix C: Chapter 4................... 165

D Appendix D: Chapter 5 . . . . . . . . . . . . . 183

$\begin{array}{ll}\text { Bibliography } & 191\end{array}$ 


\section{List of Figures}

1.1 New Zealand tectonics . . . . . . . . . . . . . . . . . 6

1.2 Regions of study in North Island . . . . . . . . . . . . . . . 8

1.3 Locations of slow slip events and interface coupling on the Hikurangi subduction zone . . . . . . . . . . . . . . 10

1.4 Schematic cross-sections of Hikurangi margin . . . . . . . . . 12

1.5 GeoNet earthquake locations in North Island . . . . . . . . . . . . . 15

1.6 Seismic wave propagation through anisotropic material . . . . . . . 16

1.7 Stress-induced vs structural anisotropy . . . . . . . . . . . . 23

1.8 Stress-induced alignment of microcracks . . . . . . . . . . . . . . 24

1.9 Previous SKS teleseismic shear wave splitting results in North Island 30

2.1 GeoNet, RATTIL, and HOBITSS station locations . . . . . . . . . . . 34

2.2 Example of waveform using P-wave orientation technique . . . . 36

2.3 Example of waveform using S-wave orientation technique . . . . 38

2.4 Example of an A grade shear wave splitting result using MFAST . . 42

2.5 Example of a B grade shear wave splitting result using MFAST . . . 43

2.6 MFAST workflow . . . . . . . . . . . . . . . . . . 45

2.7 Travel paths of teleseismic XKS phases . . . . . . . . . . . . . 46

2.8 SplitRacer workflow . . . . . . . . . . . . . . . 47 
3.1 HOBITSS and GeoNet station locations and previous SWS results . 51

3.2 Gisborne regional earthquake locations . . . . . . . . . . . . . 56

3.3 Rose diagrams of fast azimuths in HOBITSS region . . . . . . . . . 59

3.4 Spatial averaging of SWS fast azimuths . . . . . . . . . . . . 61

$3.5 V_{P} / V_{S}$ and SWS time variations at HOBITSS stations . . . . . . . 62

3.6 Temporal variations in $V_{P} / V_{S}$ and delay time on stack of offshore stations .......................... 65

3.7 Fluid flow schematic model . . . . . . . . . . . . . . . . . . . 68

4.1 Locations of GeoNet seismic stations in central North Island . . . . 77

4.2 Total slip for the 2010 Manawatu slow slip event . . . . . . . . . . . 79

4.3 Gravity and GPS strain rate field . . . . . . . . . . . . . . . 82

4.4 Faulting kinematics relative to principal stress directions in the Earth's crust ........................ 83

$4.5 S_{H} \max$ directions and subduction coupling $\ldots \ldots \ldots$. . . . . . 84

4.6 GeoNet earthquake locations in central North Island . . . . . . . . . 86

4.7 Raypath coverage using quadtree gridding . . . . . . . . . . . . 88

4.8 Example of temporal analysis at station BFZ . . . . . . . . . . . 92

4.9 Rose diagrams of fast polarization azimuths in central North Island 93

4.10 Spatial averaging of SWS fast azimuths from TESSA in central North Island . . . . . . . . . . . . . . . . . . . . . 96

4.11 Shear wave splitting parameters versus depth at station ANWZ . . 97

4.12 Quantitative comparison of fast azimuths to other measurements . 99

4.13 Histogram and scatter plots for fast azimuth comparative analyses 100

4.14 Spatial averaging of shear wave splitting delay times . . . . . . . . 104

4.15 Fault-derived $S_{H \max }$ and $S_{H} \max$ from focal mechanisms compared to SWS fast azimuths . . . . . . . . . . . . . . . . . . 106 
5.1 RATTIL network station locations _ . . . . . . . . . . . . . 115

5.2 Crustal structure profile across the Taranaki Ruapehu Line . . . . 116

5.3 Olivine fabric diagram . . . . . . . . . . . . . . . . . . 119

5.4 Previous SKS splitting measurements in North Island . . . . . . . . 121

5.5 Deployment timeline for RATTIL stations . . . . . . . . . . . . . . . 122

5.6 Distribution statistics of teleseismic earthquake results . . . . . 123

5.7 Example of a "good" shear wave splitting measurement using SplitRacer . . . . . . . . . . . . . . . . . 125

5.8 Histogram of earthquake magnitudes by result category . . . . . . 127

5.9 Rose diagrams of mean SWS fast azimuths at each RATTIL station 129

5.10 Average delay times at RATTIL stations . . . . . . . . . . . . 130

5.11 SKS record section for a single earthquake on RATTIL stations . . . 131

5.12 Station delay times plotted with TRL structural cross section . . . . 132

5.13 Mantle flow models . . . . . . . . . . . . . . . . . . . . . 135

A.1 Rayleigh wave orientation results . . . . . . . . . . . . . . . . . . . 149

B.1 $V_{P} / V_{S}$ time series for stations with timing issues $\ldots \ldots \ldots$

B.2 Earthquake raypaths for HOBITSS region . . . . . . . . . . . . 159

B.3 Comparison of $V_{P} / V_{S}$ and delay time for different station stacks . . 160

B.4 Examination of earthquake cluster affecting $V_{P} / V_{S} \ldots \ldots \ldots$

B.5 Spatial sub-catalogs of earthquakes . . . . . . . . . . . . . . 162

B.6 $V_{P} / V_{S}$ and delay time results for earthquake sub-catalogs $\ldots \ldots 163$

B.7 Angle of incidence versus $V_{P} / V_{S}$ ratio $\ldots \ldots \ldots \ldots \ldots$

C.1 Temporal variations in SWS parameters for GeoNet stations in central North Island . . . . . . . . . . . . . . . . . . . . . 167 
C.2 cGPS time series for GeoNet stations in central North Island . . . . 170

C.3 Spatial averaging depth comparison for GeoNet stations in central North Island . . . . . . . . . . . . . . . . . . . . . 173

C.4 Fast azimuth and delay time versus depth for GeoNet stations in central North Island . . . . . . . . . . . . . . . . . . . . . . . . 174 


\section{List of Tables}

1.1 Anisotropic symmetry systems of Earth's minerals . . . . . . . . . . 19

2.1 Ocean-bottom orientation results . . . . . . . . . . . . . . 40

3.1 Average $\Phi, V_{P} / V_{S}$, and delay time for HOBITSS and GeoNet stations 63

4.1 Average $\Phi, V_{P} / V_{S}$, and delay time for GeoNet stations in central North Island . . . . . . . . . . . . . . . . . . . . . . . . . . . 94

5.1 Average $\Phi$ and delay time for RATTIL stations . . . . . . . . . . . 126

A.1 Information for earthquakes used in P and Rayleigh wave analyses 146

B.1 Velocity model used for MFAST in HOBITSS region . . . . . . . . . 156

C.1 Velocity model used for MFAST in central North Island region . . . 166

D.1 Individual SWS results on RATTIL stations . . . . . . . . . . . . . 184

D.2 SWS results for measurements from earthquakes repeating on more than one RATTIL station . . . . . . . . . . . . . . . . . . 188 


\section{Chapter 1}

\section{Introduction and background}

\subsection{Introduction}

The study of seismic anisotropy is dependent on the fact that deformation (due to stress or strain) creates a preferred orientation in materials, causing waves to travel faster in one direction than another (Crampin and Chastin, 2003; Savage, 1999). Seismic anisotropy in the Earth can arise from a number of causes, such as stress-induced alignment of cracks, alignment of anisotropic crystals, structures (e.g., fault fabric), and lithology. Shear wave splitting (SWS) provides us with a tool to examine anisotropy beneath the surface and better reflects the dynamic processes at depth. SWS allows us to determine the magnitude and direction of seismic anisotropy and is a widely used technique to understand the processes affecting the crust and upper mantle. The increased number of anisotropy studies at subduction zones draws attention to a large variety of results and interpretations. This suggests that every subduction zone is unique and has to be evaluated individually. Due to various factors influencing seismic anisotropy at subduction zones, more research is required to better understand the complexity of these environments. (Webb et al., 2020)

Covered by permanent and transient seismic networks, the North Island of New Zealand provides us with an excellent laboratory to study plate boundary deformation processes. Several SWS studies have been conducted in Hikurangi subduction zone in North Island, but few cover the region in high detail, leaving large areas of North Island unstudied. In this thesis, we investigated seismic anisotropy 
measured by SWS across the subduction zone in North Island. This was carried out as three studies in both on-shore and offshore experiments. In the first study, we focused on temporal and spatial variations in crustal anisotropy during slow slip events (SSEs), offshore eastern North Island. In the second study, we analyzed spatial variations in crustal anisotropy across central North Island. And finally, in the third study we investigated upper mantle anisotropy along the TaranakiRuapehu Line (TRL) in the back-arc region of western North Island. Together, these three studies provide an extensive SWS analysis of North Island that helps to better understand the relationships between anisotropy and crustal and upper mantle deformation. In particular, this thesis addresses the following questions:

1. A) What SWS parameters are observed at the shallowest portion of the subducting thrust, offshore the East Coast of the northern Hikurangi subduction zone? B) How does crustal anisotropy vary on-land across North Island? C) How do SWS parameters vary offshore, as opposed to on-land? D) How can we differentiate between areas of stress-induced and structurally-controlled anisotropy in the crust?

2. Does anisotropy vary temporally during slow slip? What are the mechanics causing these variations?

3. Do SWS parameters from teleseismic phases change across the TaranakiRuapehu Line and do they vary with depth? If so, what are the sources controlling the observed changes?

\subsection{Thesis content}

This thesis consists of six chapters. The first two chapters present background information and methodology. Chapters 3,4 and 5 describe three self-contained studies that represent the main body of the thesis. The first person plural writing style ("we") was used in Chapters 3, 4 and 5, as is common practice for publications involving several authors. The three studies are at various stages of preparation for publication in academic journals and contain individual abstracts; introductions; methods and conclusion sections. Chapter 3 has been published in "Earth and Planetary Science Letters" (Zal et al., 2020). This thesis is structured as follows: 


\section{Chapter 1: Introduction and background}

Chapter 1 gives an introduction to this thesis and contains a geological and geophysical review of the literature and an introduction to seismic anisotropy that is relevant to the interpretations of Chapters 3,4 and 5 .

\section{Chapter 2: Methods}

Chapter 2 introduces the data and methods used in Chapters 3, 4 and 5. In one section of this chapter, we describe the methods used to find the orientations of the ocean-bottom seismometers required for analyses in Chapter 3. This is followed by descriptions of the SWS techniques used to determine crustal and upper mantle anisotropy, the main tool for analyses in all three studies. SWS analysis on local earthquakes is performed in Chapters 3, 4 and 5 using the Multiple Filter Automatic Splitting Technique (MFAST) from Savage et al. (2010b). SWS analysis on teleseismic earthquakes is performed in Chapter 5 using the SplitRacer method (Reiss and Rümpker, 2017). Supplemental materials for this chapter can be found in Appendix A.

Chapter 3: Temporal and spatial variations in seismic anisotropy and $V_{P} / V_{S}$ ratios in a region of slow slip

Chapter 3 builds on the success of the Hikurangi Ocean Bottom Investigation of Tremor and Slow Slip (HOBITSS) experiment, whose array of ocean bottom instruments captured, for the first time, a large shallow slow slip event offshore the East Coast of North Island in October 2014. We used HOBITSS and GeoNet data to examine temporal and spatial variations in crustal anisotropy during this uniquely recorded SSE. Our objective of understanding the physical environment that hosts slow slip complements similar efforts in Cascadia and Japan. Our results demonstrate that SWS and $V_{P} / V_{S}$ are effective tools for investigating stress changes and fluid movements associated with slow slip.

The work presented in Chapter 3 was published in Earth and Planetary Science Letters as: "Temporal and spatial variations in seismic anisotropy and $V_{P} / V_{S}$ ratios in a region of slow slip", with myself (Hubert Zal) as the lead author and Katrina Jacobs, Martha Savage, Jefferson Yarce, Stefan Mroczek, Kenny Graham, Erin K. Todd, Jenny Nakai, Yuriko Iwasaki, Anne Sheehan, Kimihiro Mochizuki, Laura 
Wallace, Susan Schwartz, Spahr Webb, and Stuart Henrys as co-authors. Supplemental materials, as submitted to EPSL, for this chapter can be found in Appendix B.

\section{Chapter 4: Spatial variations in seismic anisotropy in central North Island, New} Zealand

The objective of Chapter 4 was to investigate the relationship between seismic anisotropy and the stress state across central North Island, New Zealand using one of the largest datasets of SWS measurements. We used GeoNet data to examine spatial variations in crustal anisotropy. We compared our SWS measurements with both stress models and other potential factors to elucidate contributing sources of influence controlling our measured anisotropy. Our results suggest a complex pattern of stress-induced and structural control on anisotropy across central North Island. We observe a strong structural control on anisotropy from the fault fabric along the North Island Dextral Fault Belt (NIDFB) and variations from NE-SW fault belt orientations suggest stress-induced anisotropy. Supplemental materials for this chapter can be found in Appendix C.

\section{Chapter 5: Upper mantle anisotropy across the Taranaki-Ruapehu Line in North Island, New Zealand}

In Chapter 5, we investigated the relationship between seismic anisotropy and mantle deformation across a deep boundary in the mantle, marked by the TaranakiRuapehu Line (TRL), in the back-arc region of western North Island, New Zealand. Numerous geophysical studies have examined changes across the TRL, such as in electrical conductivity, gravity, seismic attenuation, and crustal thickness, but few studies have provided constraints on the upper mantle structure from seismic anisotropy. We used data recorded on several broadband seismometers from the Ruapehu and Taranaki Teleseismic Imaging Line (RATTIL) to study upper mantle anisotropy. Our measurements reveal a strong NE-SW oriented anisotropy across the TRL, likely due to the NE-SW lattice-preferred orientation of olivine in the mantle wedge due to shear deformation associated with oblique convergence and trench-parallel mantle flow. Teleseismic SWS results suggest that the transition to the apparent isotropy observed in previous studies in this region is located north of the RATTIL array (north of approximately $-38.75^{\circ}$ ). Supplemental materials for 
this chapter can be found in Appendix D.

\section{Chapter 6: Synthesis and conclusions}

In the final chapter, the key results of the three studies are examined and discussed together. The discussion extends to the implications for future research.

\subsubsection{Statement of contributions}

The research described in this thesis has been conducted by me (Hubert Zal), unless stated otherwise. Jefferson Yarce and Anne Sheehan contributed the earthquake database used in Chapter 3. Seismic data from ocean-bottom instruments came from Laura Wallace and Kimihiro Mochizuki (Chapter 3). We thank Kimi Mochizuki and the Earthquake Research Institute at the University of Tokyo, Japan for providing Hubert Zal and Martha Savage with fellowships as visiting researchers and stimulating valuable discussion that significantly improved the manuscript. Katrina Jacobs, Martha Savage, Jefferson Yarce, Stefan Mroczek, Kenny Graham, Erin K. Todd, Jenny Nakai, Yuriko Iwasaki, Anne Sheehan, Kimihiro Mochizuki, Laura Wallace, Susan Schwartz, Spahr Webb, Stuart Henrys, Emily-Warren Smith and Carolyn Boulton provided feedback on the manuscript (Chapter 3). Simon Lamb and Hamish Hirschberg provided guidance on statistical analysis and stress models (Chapter 4). Jesse-Lee Dimech and Tim Stern provided data used for teleseismic SWS (Chapter 5). My supervisors, Martha Savage and Katrina Jacobs, provided guidance and advice throughout the thesis.

\subsection{North Island, New Zealand}

\subsubsection{Tectonic setting of North Island, New Zealand}

New Zealand lies along a complex and active boundary between the Australian and Pacific tectonic plates (Figure 1.1). East of North Island, the Pacific plate subducts at a low angle beneath the Australian plate along the westward dipping Hikurangi subduction zone (e.g., Walcott, 1984). The plates converge through the North Island region at $<40-45 \mathrm{~mm} / \mathrm{yr}$, at an oblique angle to the orientation of the plate boundary (DeMets et al., 1994; Beavan et al., 2002; Altamimi et al., 2012). Unlike most subduction margins around the world, the shallowest part of the 


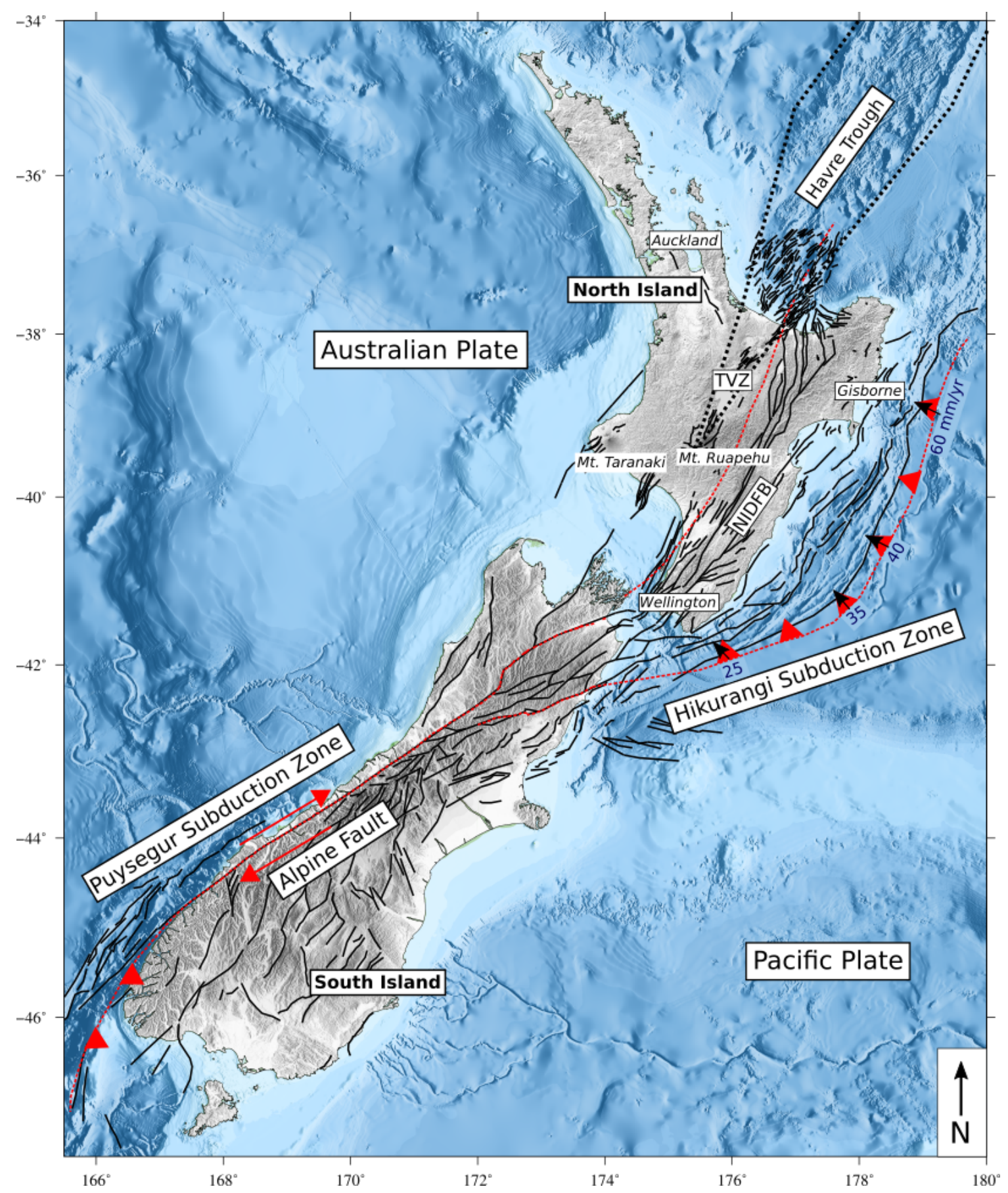

Figure 1.1: Overview of New Zealand tectonics. The New Zealand portion of the Australian and Pacific plate boundary (red) is marked by the Hikurangi Margin in the North Island and the Alpine Fault in the South Island (Coffin et al., 1998). Other major features of the North Island are the Taupo Volcanic Zone (TVZ), the extent of which is marked by the dashed-blacked lines and active onshore and offshore faults (black) throughout the North and South Island (https://data.gns.cri.nz/af/). Numbers along the Hikurangi subduction margin represent slowing subduction rates towards the south (Wallace et al., 2012a). 
subduction thrust is located within $\sim 40 \mathrm{~km}$ of the coastline. The Pacific plate subducts at a dip of just $12^{\circ}-25^{\circ}$ beneath the eastern North Island and is only $\sim 20$ $\mathrm{km}$ below the surface just north of Wellington and $\sim 15 \mathrm{~km}$ beneath the Gisborne area (Williams et al., 2013). Slip on the Hikurangi subduction interface is partitioned, with the margin parallel component largely accommodated by strike slip faulting in the upper-plate North Island Dextral Fault Belt (NIDFB; Beanland and Haines, 1998; Wallace et al., 2004).

The three areas covered in this thesis are shown in Figure 1.2 and their general geological features are described in the following sections. More detailed descriptions of the specific geological and geographic features of individual study areas are given in individual chapters.

\section{Raukumara Peninsula and Ranges}

The Raukumara Peninsula and Ranges lie along the northeastern tip of North Island (Box 1, Figure 1.2). While the the basement rock beneath the Raukumara Peninsula is formed of highly faulted and folded Torlesse Greywacke, of late Jurassic to early Cretaceous, the northeastern ranges are covered by the East Coast allochthons (ECA), Cretaceous to Oligocene rock (Mortimer, 2004). The ECA sequence of sedimentary and igneous rocks were thrust atop basement rock in early Miocene. Above the ECA and across the eastern half of the Peninsula outcrops shallow marine deposits of limestone, mudstone, and sandstone, called the Neogene sedimentary rock (Mortimer, 2004). Faulting in the Raukumara Peninsula comprises a mixture of north and northwest striking normal faults and east-west dextral-normal faults (Langridge et al., 2016) which accommodate the geodetic strain in the region. The clockwise rotation of the Raukumara Peninsula results in a three-fold increase in convergence rate at the northern Hikurangi Margin, $\sim 60 \mathrm{~mm} / \mathrm{yr}$, compared to $\sim 20 \mathrm{~mm} / \mathrm{yr}$ at the southern Hikurangi Margin (Wallace et al., 2012a).

\section{Taupo Volcanic Zone and central North Island}

The subducting Pacific plate beneath the Australian plate creates an extensional back-arc system known as the Taupo Volcanic Zone (TVZ; Figure 1.2). The TVZ is considered as the southern, onshore extension of the Havre Trough, an extending basin (Walcott, 1984). The faults in this region mainly strike NNE-SSW, with ex- 


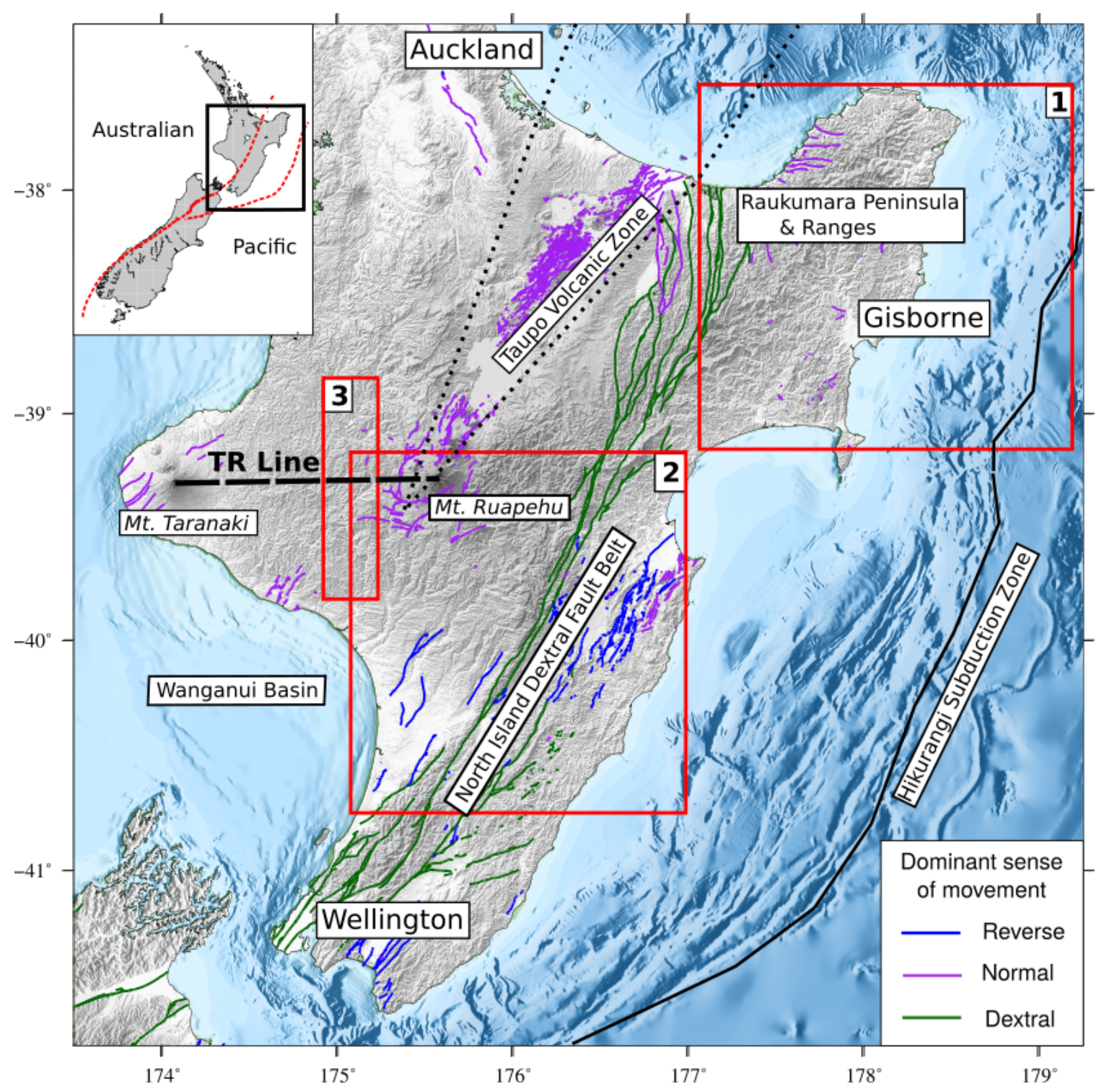

Figure 1.2: The three study areas in North Island, New Zealand. Active fault traces are from Langridge et al. (2016).

ception of the faults around Mt. Ruapehu. Northeast of Mt. Ruapehu, the average strike of the faults is $030^{\circ}$ (Rowland and Sibson, 2001), while faults just south of Mt. Ruapehu predominately strike closer to E-W.

The clockwise rotation of the Raukumara Peninsula produces contraction of the upper plate south of the TVZ and back-arc rifting in central North Island (Walcott, 1984). North Island's main geological features, such as the Raukumara mountain ranges, the North Island Dextral Fault Belt, and the TVZ, tend to follow a trench parallel, NNE-SSW, direction (Beanland and Haines, 1998). 


\section{Taranaki-Ruapehu Line}

Southwest of the TVZ the eastward trending geophysical boundary known as the Taranaki-Ruapehu Line (TRL), is oriented E-W, unlike the NE-SW trending structures of the Hikurangi margin (Hatherton, 1970; Mooney, 1970). The TRL was first recognized more than 40 years ago as a deep boundary in the mantle at which seismic attenuation (Q-1) changes abruptly (Hatherton, 1970; Mooney, 1970). Several studies have suggested the presence of a 7-10 $\mathrm{km}$ crustal step across the TRL (Stern et al., 1987; Seward et al., 2009; Salmon et al., 2011; Tozer et al., 2017; Dimech et al., 2017). It has been suggested that this step in the crust and Moho is a result of delamination of the lower crust and mantle lithosphere to the north (Stern et al., 2013; Dimech et al., 2017). An estimated crustal thickness of $\sim 32 \mathrm{~km}$ (Salmon et al., 2011) south of the TRL thins to just $25 \mathrm{~km}$ (Stratford and Stern, 2006; Salmon et al., 2011) north of the line. Extensional tectonics are dominant north of the TRL, while compressional tectonics dominate south of the line. At the western end of the TRL lies Taranaki, an andesitic volcano. The Taranaki basin, located north of Taranaki volcano, is characterized by NE-SW oriented normal faults induced by back-arc rifting.

\subsubsection{The Hikurangi subduction zone}

\section{Coupling across the Hikurangi subduction zone}

Wallace et al. (2004) used campaign GPS (Global Positioning System) data to measure interseismic slip rate deficits on the Hikurangi subduction interface and found slip on the interface to be non-uniform (Figure 1.3). They showed that the northern part of the Hikurangi margin is a weakly coupled zone with coupling occurring at $<10 \mathrm{~km}$ depth, while beneath southern North Island the plate boundary is interseismically coupled to depths of $25-35 \mathrm{~km}$ (Figure 1.3). A slip deficit of $\sim 20$ $30 \mathrm{~mm} / \mathrm{yr}$ on the slab interface below the southern North Island, indicates partial 'locking' or 'coupling' between the two plates. The contrast in locking behavior between the northern and southern Hikurangi is also supported by Lamb and Smith (2013) using a simple locking depth modeling approach. These zones of interseismic coupling are thought to represent areas of contemporary strain accumulation, with increased likelihood of future subduction thrust events (Wallace et al., 2004). 
While the accumulation of stress in the locked zone, to the south, could result in a mega-thrust subduction earthquake near the plate interface, the lack of coupling promotes stress distribution across the plate and encourages slow slip events to the north.

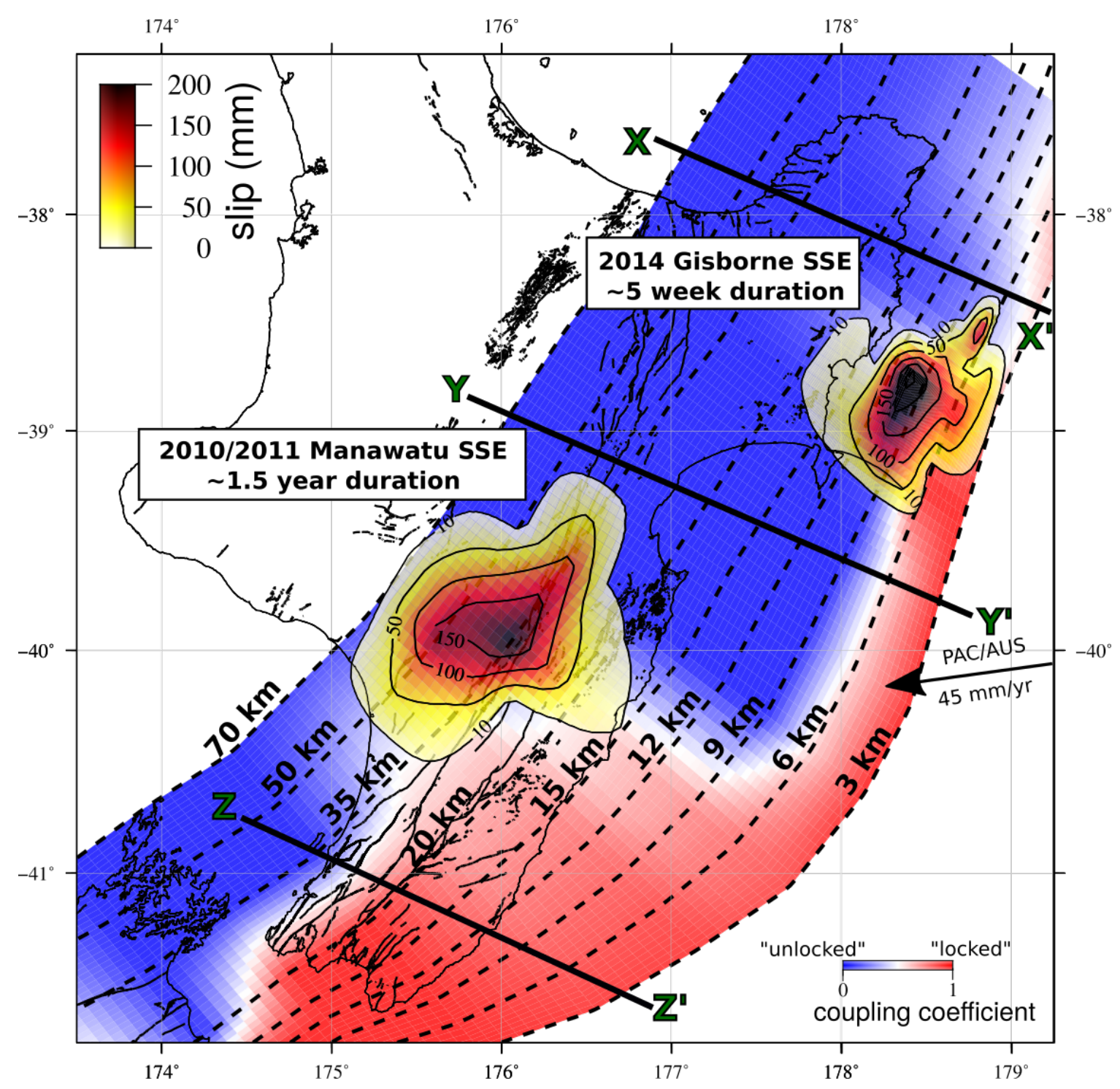

Figure 1.3: Subduction interface and interseismic coupling for the Hikurangi subduction zone [after Wallace et al. (2004)]. Locations of the 2010/2011 Manawatu SSE (slip contours from Wallace et al. (2012b)) and the 2014 Gisborne SSE (slip contours from Wallace et al. (2016)). Thick black lines show locations of schematic cross sections $X-X^{\prime}, Y-Y^{\prime}$, and Z-Z' in Figure 1.4. Dotted contours of plate interface (in $\mathrm{km}$ ) are from Williams et al. (2013). The black arrow shows the convergence rate between the Pacific and Central Hikurangi blocks [from Wallace et al. (2004)]. 


\section{Slow slip events}

Slow slip events (SSEs) are characterized as slow earthquake-like events that release energy over periods ranging from days to years. SSEs have been detected and researched in many subduction systems around the world, with most SSEs occurring in the circum-Pacific Rim including Cascadia (United States), southern Mexico, Costa Rica, Japan, and New Zealand's North Island (e.g., Schwartz and Rokosky, 2007; Peng and Gomberg, 2010). In many cases, SSEs have been associated with increased seismicity and large earthquakes, like the 2011 Tohoku megathrust event (Mw 9; e.g., Uchida et al., 2016). The understanding of physical processes occurring during SSEs is particularly important from an earthquake hazard perspective, given the possibility that a SSE could either set off a large earthquake within a nearby area of the plate (e.g., Obara and Kato, 2016) or alternatively be triggered by earthquakes (e.g., Wallace et al., 2018).

Over the last two decades, the North Island of New Zealand has been a major focus of research for investigating SSEs. Since 2002, more than 30 slow slip events have been documented and observed in New Zealand using continuous GPS (cGPS; e.g., Douglas et al., 2005; Wallace and Beavan, 2010; Wallace et al., 2012b; Wallace and Eberhart-Phillips, 2013; Wallace et al., 2016). The coupling of the plate interface plays an important role in the generation of SSEs in New Zealand (Figure 1.4). The driving mechanisms behind the occurrence and nucleation of SSEs are still poorly understood, but observational and modelling studies suggest that SSEs arise in the transition zone between velocity strengthening (i.e., aseismic creep) and velocity weakening (i.e., stick slip) behavior on the plate interface, possibly in the presence of high fluid pressures (e.g., Audet et al., 2010). The SSEs in North Island have revealed diverse characteristics including variations in duration, from six days to 1.5 years, equivalent moment release from $\mathrm{Mw}$ 6.3-7.2, depths from 2-60 km, and recurrence intervals spanning from two to five years (Wallace et al., 2012b). One of the differences between SSEs occurring in the Manawatu region and those further north, in the Raukumara region, lies in their depth along the subduction interface. The central North Island SSEs occur at deeper coupling depths, while coupling further north, in the Raukumara region occurs only at shallow depths (Wallace and Beavan, 2006). It is suggested that the main difference in the recurrence and duration characteristics between the southern and northern SSEs 
is due to the different depths at which they occur $(25-60 \mathrm{~km}$ vs. $<14 \mathrm{~km}$; Wallace and Beavan, 2006).
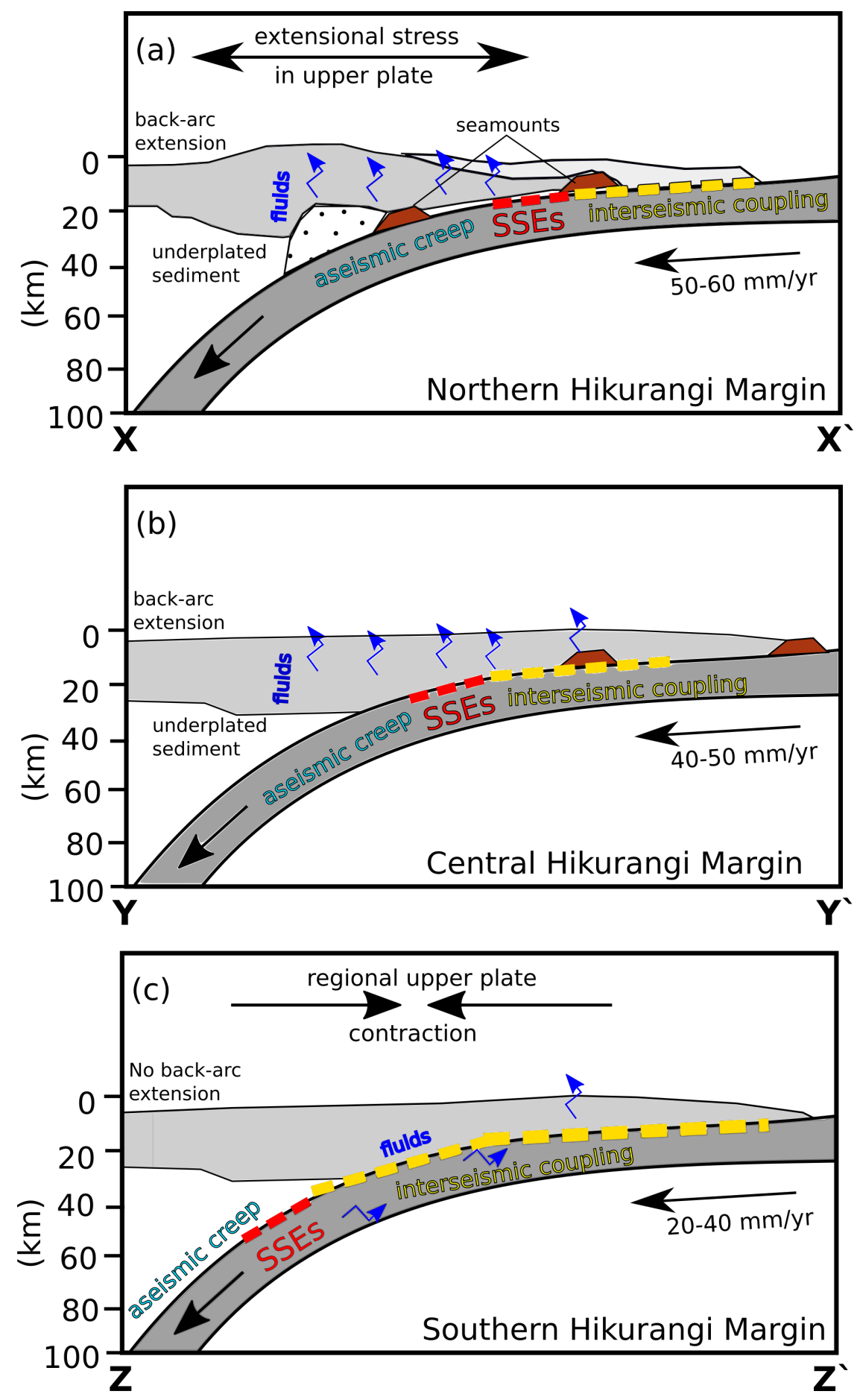

Figure 1.4: Schematic cross-sections, modified from Wallace et al. (2009), showing main processes that may occur in the (a) northern (b) central and (c) southern Hikurangi margin. Red dashed lines show approximate locations of slow slip events and yellow dashed lines mark approximate locations of interseismic coupling. Locations of cross sections are given in Figure 1.3. 
The northern part of North Island hosts some of the shallowest, best documented SSEs in the world. The Gisborne SSEs are of particular interest due to their shorter recurrence intervals (1.5-2 years), short duration (several weeks) and large horizontal deformation signals (1-3 cm onshore). The large, frequent nature of the northern Hikurangi SSEs make it an ideal location to deploy seafloor geodetic instruments and ocean bottom seismometers (OBSs) to capture seafloor deformation and seismicity related to offshore SSEs. The September-October 2014 Gisborne SSE is the first in New Zealand to be recorded by offshore instruments from the Hikurangi Ocean Bottom Investigation of Tremor and Slow Slip (HOBITSS) experiment (Wallace et al., 2016). The SSE lasted five weeks, from late-September to mid-October and occurred directly below the ocean bottom recorders of the HOBITSS experiment (Figure 1.3). We examine the September-October 2014 Gisborne SSE in closer detail in Chapter 3. The best fitting slip model shows large slip (10 to $20 \mathrm{~cm}$ ) between 4 and $7 \mathrm{~km}$ depth beneath the HOBITSS array (Wallace et al., 2016). The slip ( 10 cm) occurs as shallow as $3 \mathrm{~km}$ depth in some areas (Wallace et al., 2016). In the past our understanding of these shallow events has been hampered due to the lack of sea-based instruments, but with the data from the HOBITSS experiment we are able to better delineate the trenchward spatial extent of SSEs.

The largest SSEs typically occur downdip (down to $\sim 40 \mathrm{~km}$ ) of the deeper and strongly interseismically coupled regions of the southern Hikurangi margin. The longest Hikurangi SSE occurred in Manawatu in January 2004 and lasted 18 months to June 2005. Seven cGPS sites observed deformation, causing up to $36 \mathrm{~mm}$ of horizontal and $28 \mathrm{~mm}$ of vertical displacement at some sites (Wallace and Beavan 2006). Wallace and Beaven (2006) estimate that the Manawatu SSE occurred in the transition zone between creeping and stick slip behaviour. In 2010/2011, a similar Manawatu SSE occurred and was better constrained owing to an increase in landbased cGPS sites in the region (Figure 1.3; e.g., Wallace et al., 2012b). The moment release in the 2004/2005 and 2010/2011 events is similar, Mw 7.2 and 7.1, respectively. In early September 2010 a drastic increase recorded on cGPS sites, particularly in sites in the northeastern portion of the Manawatu region, coincided with the occurrence of the 4 September Mw 7.1 Darfield earthquake near Christchurch and a study by Wallace et al. (2012b) suggested that the passing seismic waves from the Darfield earthquake may have initiated this stage of slip. In 2013/2014 
an SSE occurred in the vicinity of Kapiti and may have been halted by a nearby January 2014 Mw 6.3 earthquake, and in 2014/2015 this SSE migrated to the area of the 2004/2005 and 2010/2011 Manawatu SSEs (Wallace et al., 2014). The five year gap in between these SSEs suggests a five-year recurrence interval for SSEs in the Manawatu region (Wallace et al., 2014). We examine the 2010/2011 Manawatu SSE in closer detail in Chapter 4.

\subsubsection{Seismicity of North Island, New Zealand}

The Pacific-Australian plate collision process is characterized by a strongly interlocking interface that causes large stress accumulation and strain deficit. The sudden failure, when stress exceeds the strength of faults, and the associated release of accumulated stress has generated great earthquakes that have devastated cities and caused tsunamis like the magnitude 8 earthquake that hit the Wairarapa area on 23 January 1855 (Downes and Grapes, 1999) and two major tsunamis on 26 March 1947 and 7 May 1947 (Doser and Webb, 2003). The North Island subduction zone is associated with numerous magnitude $\geq 3$ earthquakes $(\sim 10,000$ in the last decade; quakesearch.geo.org.nz; Figure 1.5). In North Island most earthquakes occur within the upper crust. The Wadati-Benioff zone shows a shallow dipping slab beneath eastern North Island, which steepens abruptly to a near-vertical plane at about 100-150 km depth beneath the middle of central North Island (Figure 1.5).

\subsection{Seismic anisotropy}

Seismic anisotropy is defined as a directionally dependent elastic response of seismic waves to an anisotropic material. Seismic anisotropy in the Earth can arise from a number of causes, making interpretation difficult, such as stress-induced alignment of cracks, alignment of anisotropic crystals, structures (e.g., fault fabric), and lithology (Crampin, 1981). Measuring seismic anisotropy makes it possible to derive information about the processes and materials influencing anisotropy.

Shear wave splitting (SWS) is a commonly used technique to explore crustal and upper mantle anisotropy. Splitting occurs when a shear wave travelling through an anisotropic medium splits into two orthogonally polarized quasi-shear waves with fast and slow polarizations (Figure 1.6). The term "quasi" indicates that the angle between the propagation and polarization directions is $<10^{\circ}$ (Savage, 1999). 


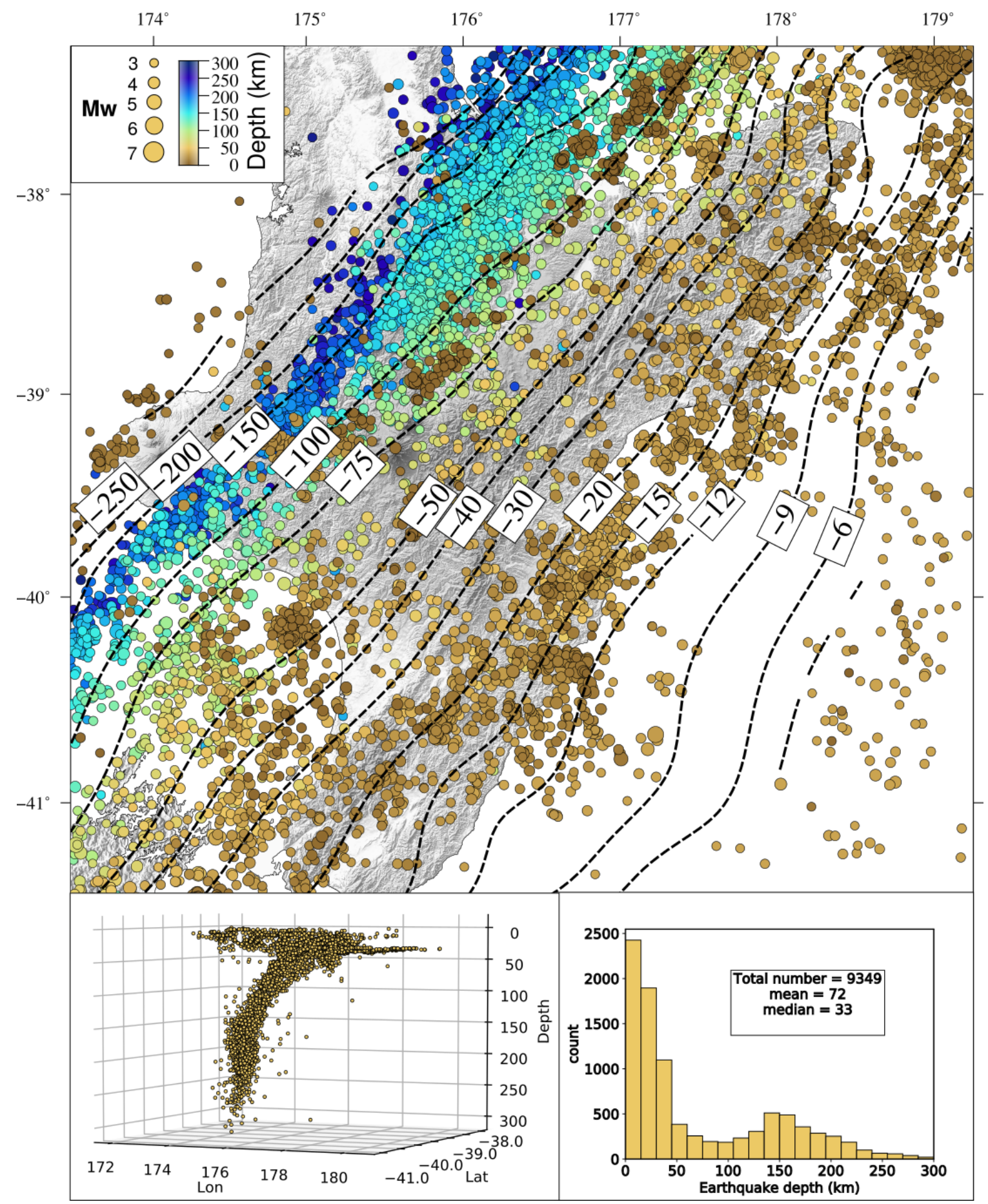

Figure 1.5: 10 years of GeoNet earthquake locations across North Island, New Zealand. Earthquake magnitudes $(\mathrm{Mw})$ are shown from 3-6.5 with depths ranging from 0-300 km (http://www.geonet.org.nz). The dotted contours of the plate interface are from Williams et al. (2013). Bottom right: histogram of earthquake depths shows that most earthquakes occur above 30 $\mathrm{km}$ depth. Bottom left: a depth cross section of earthquakes for all events between $-38^{\circ}$ and $-41^{\circ}$. 
The fast and slow waves propagate at different velocities in turn accruing a delay time, $\delta t$, the difference between the arrivals of the fast and slow waves. The magnitude of the delay time is directly dependent on the thickness of the anisotropic material and the difference between the velocity of the fast and slow polarized waves. The orientation of the fast component (also referred to as fast azimuth), $\phi$, depends on the orientation of the anisotropic medium and the travel path.

A) Vertical layering

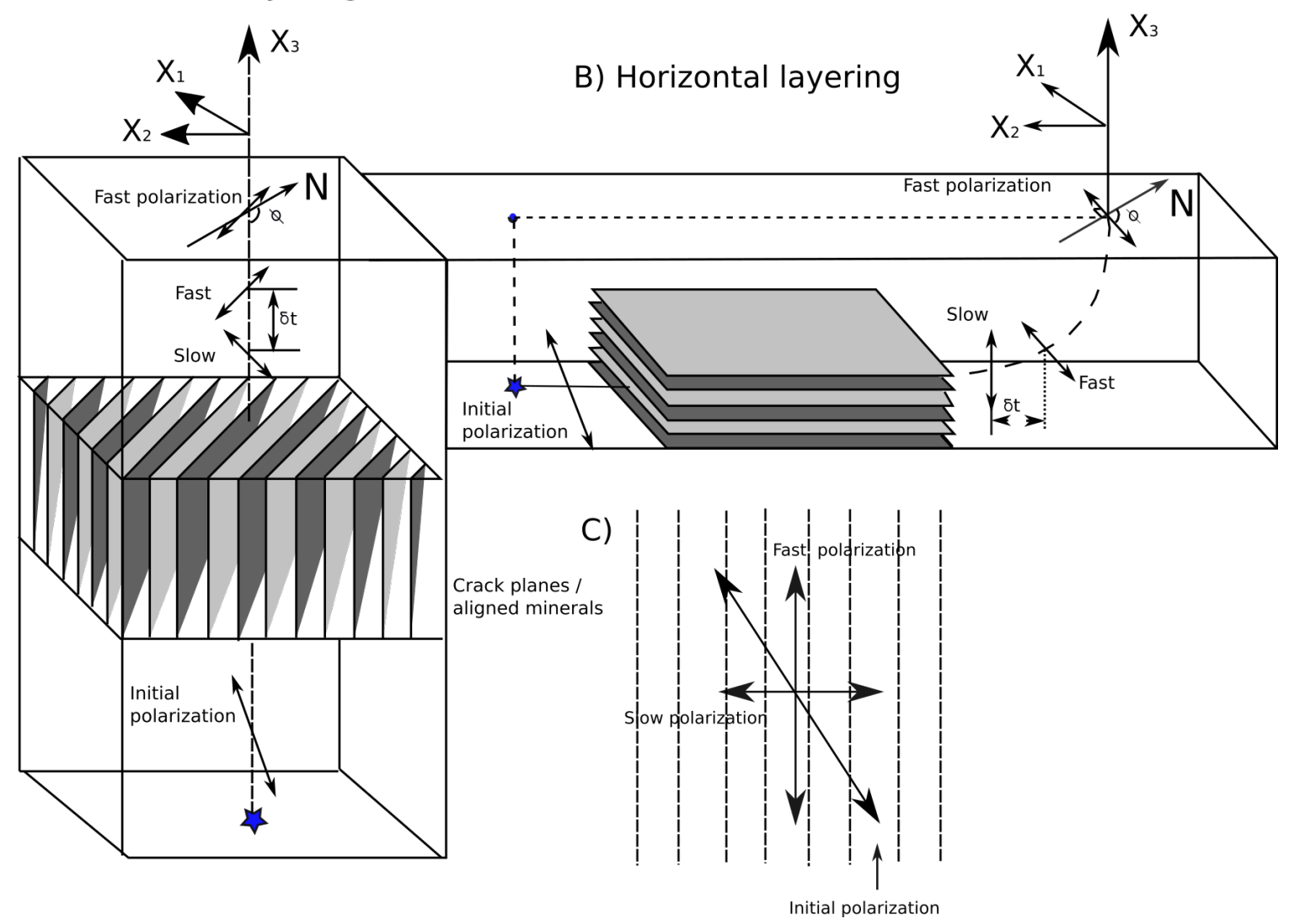

Figure 1.6: Cartoon of shear wave splitting as a seismic wave travels through an anisotropic medium [modified from Holt (2013)]. This case shows hexagonal anisotropy for (A) horizontally stacked vertical layering and for (B) vertically stacked horizontal layers. (C) Bottom right diagram shows the initial polarization before the shear wave travels through the anisotropic material and the fast and slow polarizations upon exiting the anisotropic medium.

\subsubsection{Theoretical background}

Here we summarise the theory behind shear wave anisotropy as presented in Chapter 2 of Babuska and Cara (1991). 


\section{Elastic tensors}

The basis for deriving the equations governing the propagation of a shear wave through an anisotropic medium comes from Hooke's law:

$$
\sigma_{i j}=c_{i j k l} e_{k l}
$$

where $i, j, k, l=1,2,3$. Hooke's law defines the relationship between the secondorder stress tensor $\sigma_{i j}$ and the second-order strain tensor $e_{k l}$ in an elastic medium via the forth-order stiffness tensor $c_{i j k l}$ (Means, 1976).

\section{Stress, strain and stiffness tensors}

A stress is required to deform a material. A symmetric 3D stress tensor $\sigma_{i j}$ is represented as:

$$
\sigma_{i j}=\left[\begin{array}{lll}
\sigma_{11} & \sigma_{12} & \sigma_{13} \\
\sigma_{12} & \sigma_{22} & \sigma_{23} \\
\sigma_{13} & \sigma_{23} & \sigma_{33}
\end{array}\right] .
$$

Using coordinate transformation, the above notation can be re-written so that only the diagonal elements are non-zero, representing a principal axes system where the diagonal elements are called principle stresses. It is the principal stresses that are commonly used to describe the state of stress.

Similarly, deformation is represented with the strain tensor $e_{k l}$ :

$$
e_{k l}=\left[\begin{array}{lll}
e_{11} & e_{12} & e_{13} \\
e_{12} & e_{22} & e_{23} \\
e_{13} & e_{23} & e_{33}
\end{array}\right] .
$$

The symmetry of the stress and strain tensors mean that both $i j$ and $k l$ are interchangeable. We can thus write:

$$
c_{i j k l}=c_{j i k l}=c_{i j l k}=c_{j i l k} .
$$

The symmetry between the stress and strain tensors allow for the reduction of the 
number of independent elastic coefficients from 81 to 36 , allowing for the stiffness tensor to be written as a $6 \times 6$ matrix:

$$
C_{i j k l}=\left[\begin{array}{llllll}
C_{1111} & C_{1122} & C_{1133} & C_{1123} & C_{1131} & C_{1112} \\
C_{2211} & C_{2222} & C_{2233} & C_{2223} & C_{2231} & C_{2212} \\
C_{3311} & C_{3322} & C_{3333} & C_{3323} & C_{3331} & C_{3312} \\
C_{2311} & C_{2322} & C_{2333} & C_{2323} & C_{2331} & C_{2312} \\
C_{3111} & C_{3122} & C_{3133} & C_{3123} & C_{3131} & C_{3112} \\
C_{1211} & C_{1222} & C_{1233} & C_{1223} & C_{1231} & C_{1212}
\end{array}\right] .
$$

Considering the strain energy density function $\left(c_{i j k l}=c_{k l i j}\right)$ allows us to reduce the number of independent elastic coefficients from 36 to 21 . The resulting matrix $\left(c_{m n}\right)$ can be written as:

$$
C_{m n}=\left[\begin{array}{cccccc}
C_{1111} & C_{1122} & C_{1133} & C_{1123} & C_{1131} & C_{1112} \\
& C_{2222} & C_{2233} & C_{2223} & C_{2231} & C_{2212} \\
& & C_{3333} & C_{3323} & C_{3331} & C_{3312} \\
& & & C_{2323} & C_{2331} & C_{2312} \\
& & & & C_{3131} & C_{3112} \\
& & & & & C_{1212}
\end{array}\right] .
$$

This matrix describes the most general anisotropic material with the lowest degree of symmetry (i.e., triclinic) and is the maximum number of elastic constants in any material (Table 1.1). The complexity reduces for materials with higher degrees of symmetry. In the Earth, the most commonly studied seismic anisotropy symmetries are orthorhombic and hexagonal, with 9 and 5 coefficients, respectively. For example, fluid filled cracks in the crust exhibit hexagonal symmetry, while olivine crystals in the upper mantle exhibit orthorhombic symmetry.

\section{Wave equation for anisotropic media}

Here, we derive the basic equations describing seismic body wave propagation in anisotropic media.

A plane wave propagating through a homogeneous anisotropic medium (e.g., Stein and Wysession, 2003) can be expressed as: 
Table 1.1: The number of elastic constants and examples of typical minerals for various symmetry systems [after Babuska and Cara (1991)].

\begin{tabular}{lcl}
\hline Symmetry type & Number of elastic constants & Typical mineral \\
\hline Triclinic & 21 & Plagioclase \\
Monoclinic & 13 & Hornblende \\
Orthorhombic & 9 & Olivine \\
Tetragonal & 6 & Stishovite \\
Hexagonal & 5 & Ice \\
Cubic & 3 & Garnet \\
Isotropic solid & 2 & Volcanic glass \\
\hline
\end{tabular}

$$
u(r, t)=a f(t-n, r / c)
$$

where $u(r, t)$ denotes the motion of a particle located at coordinates $r$ at time $t$, propagating in direction (unit vector $\vec{n}$ ) at phase velocity $c$ and $a$ is the vector giving the amplitude and polarity of the wave.

The equation of elastodynamics in a homogeneous anisotropic medium can be written as:

$$
\partial_{j} \sigma_{i j}=\rho \partial t^{2}\left(u_{j}\right)
$$

where $\rho$ is the density, $\partial_{j}$ represents the space derivative relative to coordinate $x_{j}$, $\sigma_{i j}$ are components of the second-order stress tensor, $\partial_{j}$ is the time derivative, and $u_{j}$ is the component of displacement in the direction $j$ of the displacement vector $u$.

By substituting Hooke's law (1.1) into (1.4) we get

$$
c_{i j k l} \partial_{j}\left(e_{k l}\right)=\rho \partial t^{2}\left(u_{j}\right)
$$

For small strain, a strain tensor can be expressed as: 


$$
e_{k l}=\frac{\frac{\partial u_{k}}{\partial x_{l}}+\frac{\partial u_{l}}{\partial x_{k}}}{2} .
$$

With the symmetry of both the strain tensor and the stiffness tensor $\left(\frac{\partial u_{k}}{\partial x_{l}}=\frac{\partial u_{l}}{\partial x_{k}}=\right.$ $\left.\partial k\left(u_{l}\right)\right)$, we can substitute equation (1.6) into (1.4):

$$
c_{i j k l} \partial_{j}\left(\frac{\frac{\partial u_{k}}{\partial x_{l}}+\frac{\partial u_{l}}{\partial x_{k}}}{2}\right)=\rho \partial t^{2}\left(u_{j}\right)
$$

which reduces to:

$$
c_{i j k l} \partial_{j} \partial_{k}\left(u_{l}\right)=\rho \partial t^{2}\left(u_{j}\right)
$$

By taking the space and time derivative of (1.3) we get

$$
\partial_{t}\left(u_{l}\right)=c^{2} a_{i}
$$

and

$$
\partial_{k} \partial_{k}\left(u_{l}\right)=a_{l} n_{j} n_{k}
$$

where $a$ are the eigenvectors and $c^{2}$ are the eigenvalues. By substituting (1.9) and (1.10) into (1.8) we get

$$
a_{l} \frac{c_{i j k l} n_{j} n_{k}}{\rho}=c^{2} a_{i}
$$

where the left side makes up the Christoffel Tensor which is given by:

$$
m_{i l}=\frac{c_{i j k l} n_{j} n_{k}}{\rho}
$$

for every propagation direction $\vec{n}$.

The eigenvalues of the Christoffel Tensor give the phase velocities of the seismic wave and the eigenvectors give the polarisation of that wave. There are three eigenvalues and three corresponding perpendicular eigenvectors which correspond to the three different wave types in a homogeneous anisotropic medium. 


\section{Plane wave in a hexagonal anisotropy system}

We provide a detailed explanation of a plane wave in a hexagonal anisotropic system, as it is the most commonly used system to understanding seismic wave propagation in different Earth media.

For the hexagonal system with the axis of symmetry parallel to the axis $x_{3}$ (in Cartesian coordinate system; e.g., Figure 1.6) the elastic tensor is given by:

$$
C_{i j k l}=\left[\begin{array}{cccccc}
C_{1111} & C_{1122} & C_{1133} & 0 & 0 & 0 \\
C_{2211} & C_{2222} & C_{2233} & 0 & 0 & 0 \\
C_{3311} & C_{3322} & C_{3333} & 0 & 0 & 0 \\
0 & 0 & 0 & C_{2323} & 0 & 0 \\
0 & 0 & 0 & 0 & C_{3131} & 0 \\
0 & 0 & 0 & 0 & 0 & C_{1212}
\end{array}\right]
$$

For the hexagonal system there are five independent coefficients $(A, C, F$, L, and $\mathrm{N}$; Love, 2013) and the corresponding elasticity matrix can be expressed as:

$$
C_{i j k l}=\left[\begin{array}{cccccc}
A & A-2 N & F & 0 & 0 & 0 \\
A-2 N & A & F & 0 & 0 & 0 \\
F & F & C & 0 & 0 & 0 \\
0 & 0 & 0 & L & 0 & 0 \\
0 & 0 & 0 & 0 & L & 0 \\
0 & 0 & 0 & 0 & 0 & N
\end{array}\right] .
$$

An isotropic medium has only two independent elements when $A=C=\lambda+$ $2 \mu, A-2 N=F=\lambda$ and $L=N=\mu$ where $\mu$ and $\lambda$ are referred to as the Lamé parameters.

An example of hexagonal anisotropy is a material with alternating fast and slow velocity layers (Figure 1.6). We provide examples for a plane wave travelling in two main directions through a layered material, in the direction perpendicular or parallel to the symmetry axis (Figure 1.6A and B). For a plane wave propagating perpendicular to the symmetry axis the Christoffel matrix yields 


$$
m_{i j}=\frac{1}{\rho}\left[\begin{array}{ccc}
A & 0 & 0 \\
0 & N & 0 \\
0 & 0 & L
\end{array}\right]
$$

This matrix has three eigenvalues $\lambda$ which correspond to three eigenvectors $a$. The eigenvectors indicate the polarization direction of the waves with varying velocities. Velocities are given by $\alpha$ or $\beta$ :

$$
\begin{aligned}
& \alpha_{1}=\sqrt{\frac{A}{\rho}}, \\
& \beta_{1}=\sqrt{\frac{N}{\rho}}, \\
& \beta_{2}=\sqrt{\frac{L}{\rho}}
\end{aligned}
$$

where $\alpha_{1}$ is the velocity of the fastest wave (P wave) which is polarized parallel to the propagation direction, $\beta_{1}$ and $\beta_{2}$ are two waves (S waves) with their polarisation's perpendicular to the propagation direction. In the case that $N>L$ it follows that $\beta_{1}>\beta_{2}$ and SWS has occurred. SWS occurs when a shear waves travelling through a given anisotropic medium splits into two orthogonally polarized quasishear waves with a fast $\left(\beta_{1}\right)$ and slow $\left(\beta_{2}\right)$ axis direction. Different polarisation's and travel paths will lead to different fast and slow axis directions.

\subsubsection{Origins of anisotropy}

Anisotropy in the Earth occurs at various depths, at different length scales, and can be controlled by different sources. Anisotropy in the crust is mainly of stress or structural origin (Babuska and Cara, 1991), while the lattice-preferred orientation (LPO) of intrinsically anisotropic minerals is the main contributor to anisotropy in the upper mantle (Kendall, 2013). Here we discuss the sources of anisotropy in the crust and upper mantle. 


\section{Crustal anisotropy}

In the crust, anisotropy is mainly caused by either the preferential closing of fluid filled (usually water or melt) microcracks at particular orientations as a response to horizontal differential stresses or by pre-existing structural features, such as faults and dikes (Babuska and Cara, 1991). These mechanisms can be referred to as stress-induced and structurally-controlled anisotropy (Figure 1.7).

Cracks in the crust can generally be approximated by a penny-shaped form with the crack normal plane perpendicular to the flat surface (Crampin and Chastin, 2003). They typically range in size from $10^{-6} \mathrm{~m}$ to a few meters, while microcracks are typically no more than a few $\mathrm{cm}$ in length (Crampin, 1981). Therefore, seismic waves, which have much larger wavelengths than the dimensions of microcracks, will sample the dominant crack orientation (Babuska and Cara, 1991).

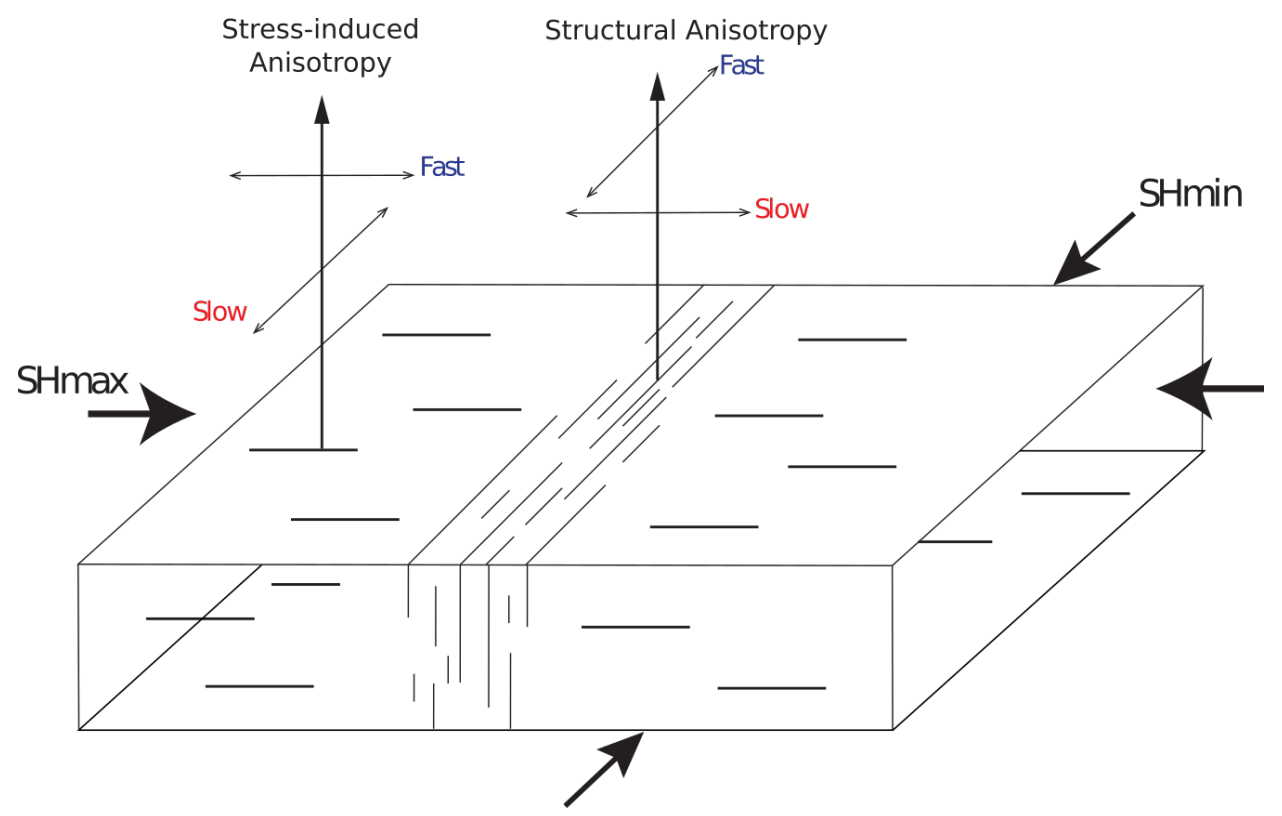

Figure 1.7: Cartoon illustrating stress-induced anisotropy adjacent to structurally controlled anisotropy (by faults) in the crust [after Boness and Zoback (2006)]. A shear-wave travelling through a stress-induced material will result in the fast azimuth parallel to the maximum compressive stress direction and a shear-wave travelling inside a fault zone will result in a fast direction parallel to the strike of the fault (fault fabric).

If these penny-shaped cracks are randomly distributed, no anisotropic signature will be present. However, if cracks are aligned, an anisotropic signature can be measured, even when the rock mass hosting the cracks is itself isotropic. Crack alignment does not mean that cracks rotate to a specific direction, instead cracks 


\section{Microcracks:}

Open

Closed

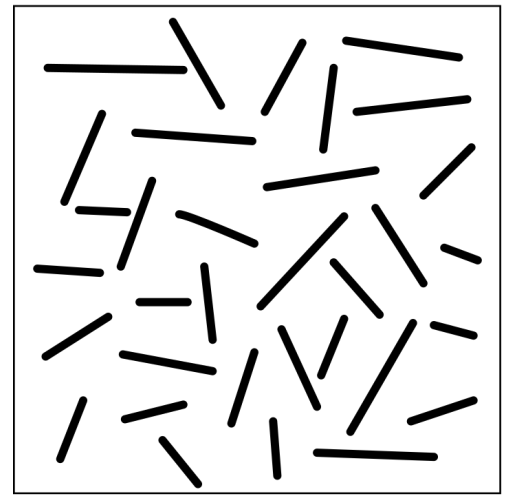

Unstressed

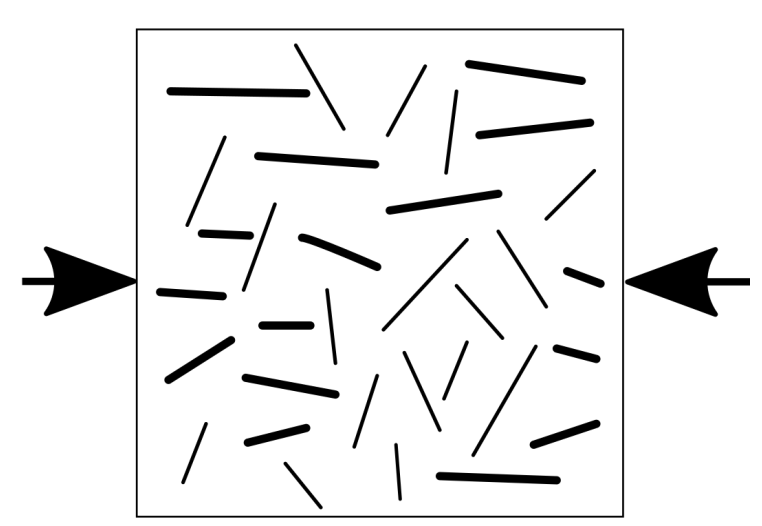

Stressed

Figure 1.8: Cartoon illustrating stress-induced alignment of microcracks. Randomly oriented open cracks are isotropic (left). In a stress regime (right) cracks which are perpendicular to the maximum compressive stress direction (represented as inward arrows) will close, leaving open cracks which are oriented sub-parallel to the maximum compressive stress.

of a certain orientation open (or remain open) and close (or remain closed) depending on the direction of applied horizontal stresses (Figure 1.8). Cracks will align perpendicular to the minimum compressive stress direction, while those perpendicular to the maximum compressive stress close (Nur and Simmons, 1969). In the upper crust, SWS fast azimuths tend to be parallel to fluid filled microcracks and to the maximum principal stress (Nur, 1971). If cracks are stress aligned then the SWS fast azimuths should show agreement with regional measurements of maximum horizontal stress. However, crustal stresses can occur on both a local scale, induced by earthquakes, and a regional scale, caused by tectonic loading. If local and regional stresses are on the same order as, or stronger than that of structural influences, and $S_{H} \max$ is not parallel to the strike of the structure, it is possible to observe changes in $S_{H} \max$ by monitoring temporal changes in SWS fast azimuth as it is sensitive to the orientation of microcracks. However, cracks tend to close completely with depth due to lithostatic pressure and so they are mainly characteristic of the mid to upper crust (Nur and Simmons, 1969). As a result, most studies of upper crustal anisotropy find little change in SWS with depth, suggesting that the top few kilometers are most important for SWS measurements (e.g., Gledhill, 1993). Although the contribution is generally small, crustal anisotropy has to be accounted for in mantle studies, as it is part of the travel path of the seismic wave. 
Stress-induced anisotropy is most common in the upper crust in areas of high fluid pressure and it has been shown that rocks under low effective stress are commonly controlled by the properties of microcracks (Wang et al., 2012). The effects of differential stress on anisotropy were observed by Nur (1971) in a study examining the preferential opening and closing of cracks in rocks in response to non-hydrostatic stresses. With increasing horizontal differential stresses cracks are compressed and become vertically aligned (Crampin, 1981). A ray traveling through vertically aligned cracks will result in maximum anisotropy and increased delay times (Nur, 1971). Cracks have also been suggested to coalesce (or link together) as stress accumulates and fractures approach criticality (Gao and Crampin, 2006). In a crackinduced anisotropic material, SWS can provide us with crucial information about crack-alignment and subsequently about stress directions.

Cracks are also important in controlling the ratio of the speed of seismic compressional and shear waves, $V_{P} / V_{S}$. With higher quantities of liquid-filled cracks, S-wave velocities are reduced more than $\mathrm{P}$ waves and hence the ratio increases (Nur, 1971). On the contrary, with higher quantities of gas-filled cracks, P-wave velocities are reduced more than $S$ waves, and the ratio decreases (Nur, 1971). Laboratory results from water saturated isotropic microcracks show that maximum $V_{P} / V_{S}$ values are achieved at low effective mean pressures (i.e., low differential stresses, high pore fluid pressures; Wang et al., 2012). Tomographic studies have shown that regions of slow-slip have elevated fluid pressures and tend to have high Poisson's ratios and high $V_{P} / V_{S}$ ratios (e.g., Audet et al., 2010).

In addition to stress-induced anisotropy, structural anisotropy is also a dominant factor in the crust. Macro-scale structures, such as fault fabrics, sedimentary bedding planes, and aligned minerals, are the main causes of structural anisotropy. In the case of structurally controlled anisotropy, the fast azimuth is usually oriented sub-parallel to the structural fabric (e.g., Savage, 1999; Zinke and Zoback, 2000; Cochran et al., 2006; Balfour et al., 2005). Large faults have been shown to influence anisotropy locally, such as the San Andreas Fault (Boness and Zoback, 2006; Li and Peng, 2017) or the North Anatolian Fault (Peng and Ben-Zion, 2004). Knowledge of the local stress field, as well as structural elements in a region makes it possible to determine whether anisotropy is controlled by stress, structure, or a combination of both mechanisms. 


\section{Upper mantle anisotropy}

Highly anisotropic crystals such as olivine constitute approximately seventy percent of Earth's upper mantle (Jeanloz et al., 1989). Within the upper mantle, strain, which may or may not be related directly to stress, creates a preferred orientation of mineral crystals, a property called lattice preferred orientation (LPO; Kendall, 2013). The strain causing the LPO of olivine crystals in the mantle is most likely induced by forces due to lithospheric plate movement over the asthenosphere or strain within the lithosphere (e.g., Savage and Silver, 1993; Park and Levin, 2002; Long and Silver, 2009). By measuring the fast azimuths using SWS the direction of maximum strain can be inferred (Savage, 1999).

Although the LPO of olivine is typically identified as the major mechanism for upper mantle anisotropy, factors such as oriented melt pockets (OMP), periodic thin layering (PTL), fluid filled cracks, dikes, and fossil anisotropy can directly influence SWS delay times and the fast azimuth polarization, obtained for the upper mantle, resulting in measurements that represent both the upper mantle and crustal anisotropy (Kendall, 2013). Although the contribution of crustal anisotropy is relatively small, with maximum delay times of 0.2-0.3 s measured in rock physics experiments (Ji and Salisbury, 1993), crustal anisotropy has to be accounted for in mantle studies, as it is part of the travel path of the seismic wave.

In certain conditions, of pressure and temperature, the $a, b$, and c axes of olivine are known to align with the extension, compression, and intermediate axes of the strain ellipse, respectively (Jung and Karato, 2001b). In type-A and type-C fabric of olivine the polarization of the faster $S$ wave will be subparallel to the flow direction (Karato et al., 2008). Type-A olivine typically occur at low stress and water-poor conditions and type- $\mathrm{C}$ fabric is observed in water-rich conditions and under low stress (Jung and Karato, 2001b). In contrast, in type-B fabric the fast azimuth will be perpendicular to the shear direction and forms under high stress (>300 $\mathrm{MPa}$ ) and high water content (Jung and Karato, 2001a), suggesting that B-type fabric of olivine can explain the presence of trench-parallel anisotropy in a region where shear direction is trench-perpendicular (e.g., Audoine et al., 2004).

Additionally, relatively new types of olivine fabric (type-D and E) have also been reported (Katayama et al., 2004). Similar to type-A and C fabrics, in type-D and type-E fabrics the fast azimuth will be subparallel to the flow direction, but type-D 
fabrics dominate at high stress and low water content while type-E fabrics occur at low stress and moderate water content (Katayama et al., 2004).

\subsubsection{Previous shear wave splitting studies}

Multiple studies of seismic anisotropy have been carried out across North Island in the last decade. These include SWS studies of the crust and upper mantle using both local and teleseismic earthquakes.

\section{Local shear wave splitting and crustal anisotropy}

Previous studies of local splitting across New Zealand have measured a dominant NE-SW fast azimuth around the northern region of South Island (e.g., Balfour et al., 2005; Karalliyadda and Savage, 2013) and across areas of North Island (e.g., Audoine et al., 2000; Evanzia et al., 2017) and have attributed this trend to pervasive shear fabrics associated with the plate-boundary deformation (Balfour et al., 2005). Several other SWS studies have also observed NE-SW trending $\left(30^{\circ}-\right.$ $60^{\circ}$ ) fast azimuths, mainly along the NIDFB in the North Island (Figure 1.9; e.g., Gledhill and Stuart, 1996; Audoine et al., 2004; Morley et al., 2006; Illsley-Kemp et al., 2019). Patterns of NE-SW trending anisotropy have been observed in Pwave anisotropy and are especially prevalent along the NIDFB (Eberhart-Phillips and Reyners, 2009). In the southern North Island, Evanzia et al. (2017) observe NESW trending anisotropy, except in the Wanganui Basin where anisotropy is trench perpendicular.

Northwest of the NIDFB, in the TVZ, a study by Morley et al. (2006) analyzed local splitting across the Central North Island Passive Seismic Experiment (CNIPSE) line (Reyners and Stuart, 2002). They were the first to observe a dramatic change in fast azimuths, observed as a strong change from trench-parallel directions in eastern North Island to trench perpendicular directions in the TVZ and northsouth directions west of the TVZ. They interpreted this as a change from crustal anisotropy in the east to uppermost mantle anisotropy in the mantle wedge under the TVZ, due to trench-normal mantle flow, to directions consistent with the apparent plate motion in the west (Morley et al., 2006). Illsley-Kemp et al. (2019) examined crustal SWS results north of Mt. Ruapehu, across the TVZ, finding dominant rift-aligned fast azimuths in the central TVZ, suggesting a strong control by 
rift-related extensional stresses, similar to directions observed by Audoine et al. (2004). Around Mt. Ruapehu, Johnson et al. (2011) observed areas where fast azimuths from SWS agree with stress estimations from focal mechanism inversions, as well as areas where fast azimuths align with fault orientations, suggesting both stress and structure-induced anisotropy.

Additionally, several studies around the world have reported temporal variations in seismic anisotropy in varying environments, such as around volcanoes, at Mt. Ruapehu (Gerst and Savage, 2004; Savage et al., 2010b) and Mt. Asama (Savage et al., 2010a), during large earthquakes, such as the 2009 L'Aquila earthquake in Italy (Lucente et al., 2010), at the Valhall oil reservoir, North Sea (Teanby et al., 2004a), and at the Rotokawa and Ngatamariki geothermal fields in New Zealand (Mroczek et al., 2019). Temporal variations in SWS fast azimuths have been previously observed at Mt. Ruapehu Volcano in North Island, New Zealand (e.g., Miller and Savage, 2001; Gerst and Savage, 2004), whereby changes in splitting were interpreted as being caused by reoriented cracks due to repeated filling or depressurizing of magmatic dike systems after the 1995-1996 eruptions (Miller and Savage, 2001; Gerst and Savage, 2004).

Unglert (2011) also searched for temporal variations in SWS during three SSEs in the northern Hikurangi between 2002 and 2010 and found a slight increase in $\delta t$ and $\sim 30^{\circ}$ of change in $\Phi$ during an SSE offshore of Gisborne in October 2002 at the closest seismic station. However, no variations were observed on more distant stations during this SSE and no changes were seen on any of the three stations during either of the other two SSEs (October 2004 and August 2009; Unglert, 2011). This suggests that SWS parameter changes during SSEs are measurable but possibly dependent on the size of SSEs and their proximity to the stations.

\section{Teleseismic shear wave splitting and upper mantle anisotropy}

Several teleseismic shear wave splitting studies have been conducted in North Island (e.g., Gledhill and Stuart, 1996; Marson-Pidgeon et al., 1999; Audoine et al., 2004; Marson-Pidgeon and Savage, 2004; Greve et al., 2008) and a compilation of some of these studies, produced by Greve et al. (2008), are shown in Figure 1.9.

Audoine et al. (2004) reported dominant NE-SW fast azimuths from SKS measurements across the northern parts of North Island. They present two possible scenar- 
ios for the TVZ and back-arc region of North Island: 1) a control by trench-parallel mantle flow if the "wet" zone ends at $130 \mathrm{~km}$ depth, related to the transpressive character of the plate boundary or 2) trench-parallel anisotropy controlled by the presence of type-B fabric of olivine LPO, if the wet zone is present from 90 to 250 $\mathrm{km}$ depth (Audoine et al., 2004). They suggest that the B fabric of olivine can explain their observed trench-parallel fast azimuths if the shear direction is trench perpendicular. However, they note that shear stresses estimated on the slab interface at $110 \mathrm{~km}$ depth are around $170 \mathrm{MPa}$ (Stern et al., 1992), which is significantly less than the value of $270 \mathrm{MPa}$ at which B fabric appears in the experiment by Jung and Karato (2001a) (Chapter 5, Figure 5.3). On the contrary, for olivine type-A fabric, the LPO of the fast olivine a-axis will align with the extension direction, and is suggested as the source of anisotropy for parts of southern North Island (Gledhill and Stuart, 1996), as well as along the eastern side of North Island (Greve et al., 2008). It has been suggested that trench-parallel mantle flow with type-A olivine beneath the slab can account for most of the observed anisotropy in fore-arc region of central North Island (Greve et al., 2008).

Greve et al. (2008) conducted splitting analyses across the entire Central North Island Passive Seismic Experiment (CNIPSE) line (Figure 1.9; Reyners and Stuart, 2002) by analyzing mantle anisotropy from teleseismic phases. They found that delay times ranged from 0.85 to $4.5 \mathrm{~s}$ across central North Island with the largest delay times in the central TVZ and the lowest delay times west of the TVZ. They observed that stations located east of the TVZ exhibited NE-SW fast azimuths with delay times around 2-3 s, with splitting parameters fairly consistent with those observed in southern North Island (Gledhill and Stuart, 1996; Marson-Pidgeon et al., 1999). The trench-parallel fast azimuths east of the TVZ were suggested to be due to the NE-SW lattice-preferred orientation of anisotropic crystals in the mantle due to trench-parallel sub-slab flow, possibly with a contribution from a trenchparallel fossil anisotropy within the slab, with a minor contribution from crustal anisotropy (0.3 s) caused by trench-parallel cracks in the overriding plate (Greve et al., 2008). Stations located in the TVZ also exhibited NE-SW fast azimuths but delay times were significantly larger (up to $4.5 \mathrm{~s}$ ), suggested to be due to the thicker mantle wedge under the TVZ (Greve et al., 2008). Greve et al. (2008) suggest that type-E olivine, in which fast azimuths will be subparallel to the flow direction, can explain the lower stresses and high delay times in the TVZ. However, the authors 


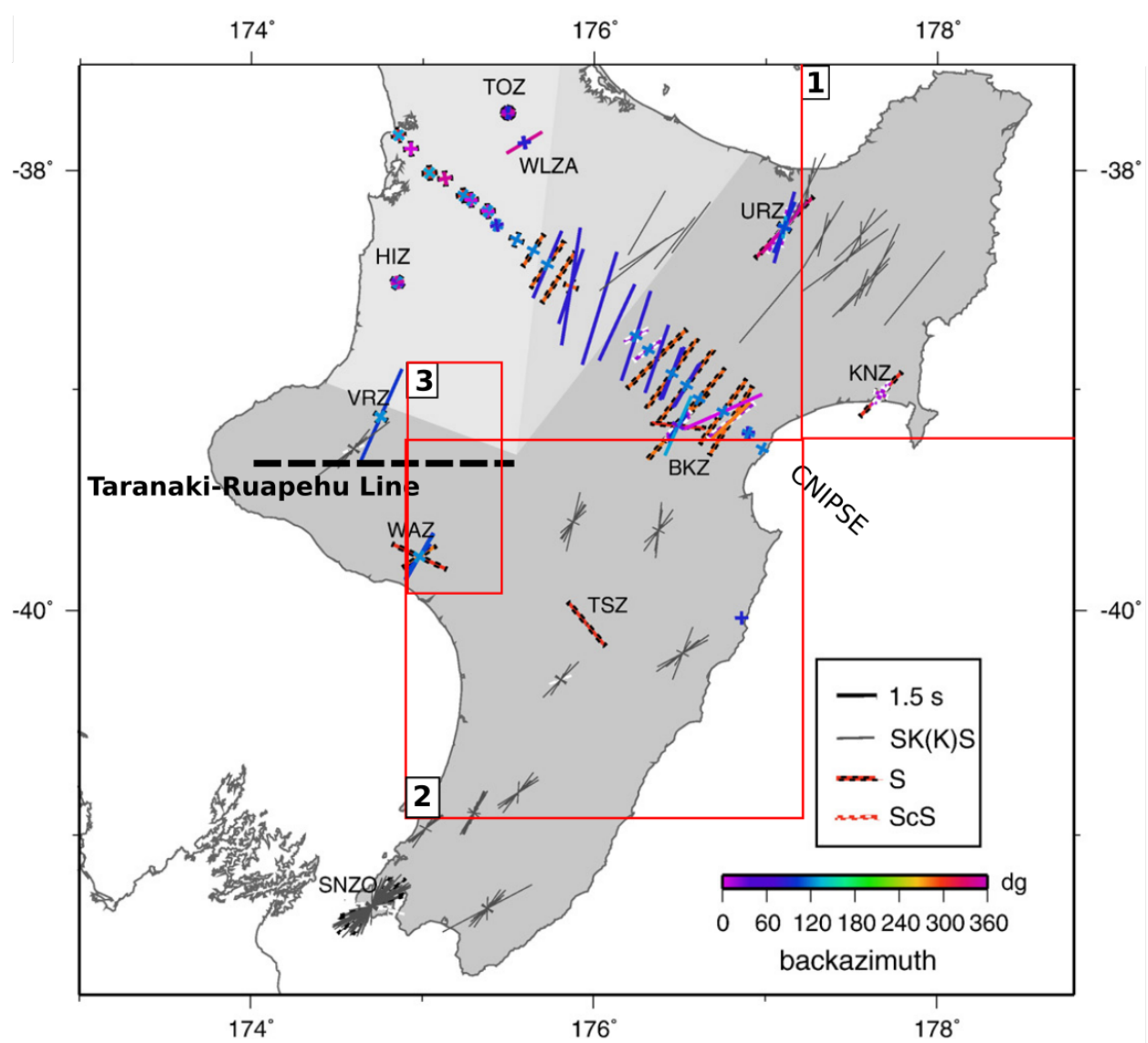

Figure 1.9: Previous splitting measurements (coloured) in North Island, New Zealand by Greve et al. (2008) and all earlier (grey) splitting measurements (Audoine et al., 2004; Cochran, 1999; Gledhill and Stuart, 1996; Marson-Pidgeon and Savage, 1997; Marson-Pidgeon et al., 1999; MarsonPidgeon and Savage, 2004) [after Greve et al. (2008)]. The lines represent each fast azimuth and the lengths are scaled according to the delay time. Each line represents a single measurement and is plotted at the corresponding station.

also propose that the high delay times can be more easily explained by a model of frequency dependent anisotropy in which the alignment of melt bands produces strong anisotropy in the extending TVZ.

Greve et al. (2008) observed that almost all stations located in western central North Island exhibited only null measurements (no apparent splitting; Figure 1.9). They suggest that the apparent isotropy observed in western North Island may be due to a vertical return flow under western North Island (Reyners et al., 2006), which can develop hexagonally symmetric anisotropy with a vertical fast axis (Savage, 1999), or from localized small-scale mantle convection. Along with a steeply dipping subducting slab under western North Island, which suggests an end of the mantle wedge dynamics, any anisotropy forming under such conditions 
would be complex and not resolvable by splitting measurements (i.e., resulting in apparent isotropy). These previous studies show a dramatic change from highly coherent NE-SW fast azimuths south of the TRL and incoherent anisotropy north of the TRL (Greve et al., 2008; Audoine et al., 2004), which is suggested to be caused by the sheared thick lithosphere to the south and an absence of the lithosphere to the north. Several teleseismic shear wave splitting studies have been conducted in North Island but few focus on the area across the TRL. We explore teleseismic SWS across the TRL in Chapter 5. 


\section{Chapter 2}

\section{Data and methodology}

\subsection{Data acquisition}

The data for this thesis were obtained from several sources, including two temporary deployments and from the permanent GeoNet network.

\subsubsection{GeoNet}

GeoNet (http://www.geonet.org.nz) is a project to build and operate a modern geological hazard monitoring system in New Zealand. It is funded by the Earthquake Commission (EQC) and designed and maintained by GNS Science and Land Information New Zealand (LINZ). For the purpose of this study, seismic data were obtained by selecting the earthquakes of interest using GeoNet's "quakesearch" (https://quakesearch.geonet.org.nz/). More information on individual GeoNet stations used for analyses can be found in corresponding Chapters 3, 4 and 5.

\subsubsection{RATTIL}

The Ruapehu And Taranaki Teleseismic Imaging Line (RATTIL) network consisted of fourteen temporary broadband seismic stations arranged north-south across the Taranaki-Ruapehu Line (TRL; Figure 2.1). These stations recorded seismic data between November 2012 and March 2014. More information about the RATTIL seismic stations can be found in Chapter 5 and Appendix D. 


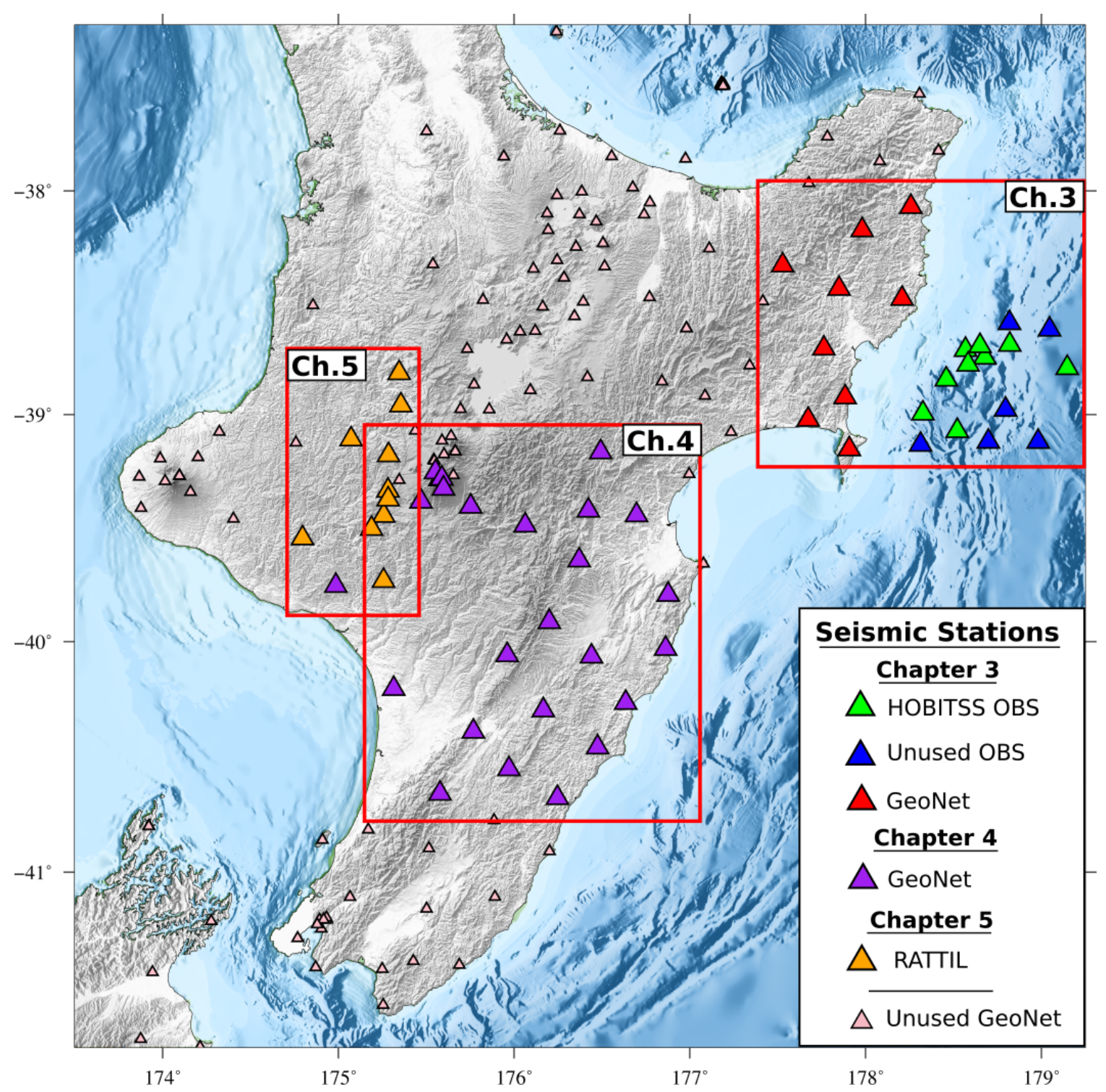

Figure 2.1: Locations of stations used in this study.

\subsubsection{HOBITSS}

The Hikurangi Ocean Bottom Investigation of Slow Slip (HOBITSS) project was part of a project funded by the National Science Foundation (NSF), the Earthquake Research Institute (ERI), University of Tokyo, and New Zealand's GeoNet project. The deployment consisted of 10 Lamont-Doherty Ocean Bottom Seismograph Instrument Pool (OBSIP) broadband ocean-bottom seismometers (LOBS) and five University of Tokyo/Earthquake Research Institute short period OBS (EOBS; Figure 2.1; Wallace et al., 2016).

The HOBITSS stations operated from May 2014 to June 2015 and during that time 
recorded 2,537 local earthquakes with magnitudes ranging from Mw 0-5. More information about the seismic stations used in this analysis can be found in Chapter 3 and Appendices A and B. Raw data from the HOBITSS experiment were downloaded from Incorporate Research Institutions for Seismology Data Management Center (IRIS-DMC) with experiment codes YH 2014-15 (seismic data). The database of earthquakes used in this study was put together by Jefferson Yarce (Yarce et al., 2019). The database is publicly available and can be found at https:/ / zenodo.org/record/2022405\#.XIbszRNKgWo and subsequently updated and published in Yarce et al. (2019).

\subsection{HOBITSS ocean bottom seismometer orientations}

The Hikurangi Ocean Bottom Investigation of Tremor and Slow Slip (HOBITSS) project recorded data from an ocean bottom seismometer array deployed from May 2014 to May 2015 at the Northern Hikurangi margin. The following section describes the steps taken to orient the ocean-bottom seismometers (OBS) required for analyses in Chapter 3 of this thesis. Unlike for terrestrial seismometers, which are easily oriented to true north during installation, the horizontal component orientations of OBSs are random and unknown upon deployment. It is therefore necessary to determine the horizontal orientation of each OBS before the full directional information of the data can be utilized. The horizontal orientations of the HOBITSS OBS receivers are determined by analyzing $\mathrm{P}$ and Rayleigh waves in a subset of raw data. Our results provide the basis for further analysis in Chapter 3 and full utilization of the data for future studies.

\subsubsection{P-wave orientation}

We identified 29 teleseismic events with magnitudes greater or equal to Mw 6.0 and distances ranging from $30^{\circ}-90^{\circ}$ from each station and from various back azimuths (Appendix A, Table A.1).

We downloaded LOBS and EOBS seismograms from IRIS in SAC format. For LOBS stations, each waveform was first band-pass filtered using a frequency of 0.04-0.1 Hz after removing the mean and trend. For EOBS stations, each waveform was high-pass filtered using a corner frequency of $0.3 \mathrm{~Hz}$ after removing the mean and trend. Next, the $\mathrm{P}$ wave arrival was picked (starting from a rough es- 

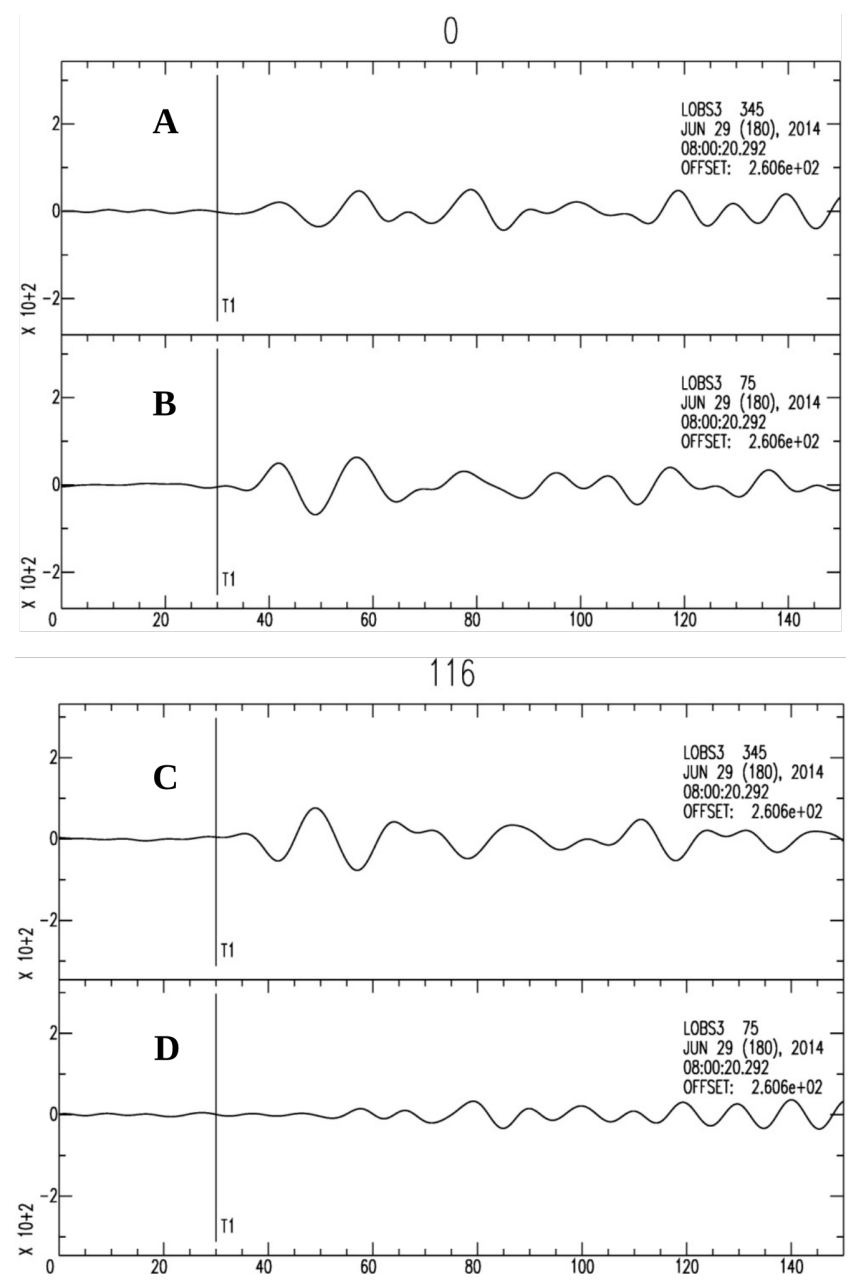

Figure 2.2: An example of an event from station LOBS3 showing the horizontal components before and after finding the orientation $\left(116^{\circ}\right)$. A) Horizontal component rotated to north. B) Orthogonal horizontal component rotated to east. C) Horizontal component rotated to $116^{\circ}$. D) Orthogonal horizontal component rotated to $116^{\circ}$.

timate based on the automatic calculated phase arrival by Taup (Crotwell et al., 1999), using the IASP91 velocity model). The time window was manually chosen to include the first appearance of the $\mathrm{P}$ wave to 2 seconds after to encompass the full phase.

We followed the transverse energy minimization method as implemented by $\mathrm{Zi}-$ etlow (2016). The horizontal seismograms were rotated and the energy on the rotated seismograms was calculated. For a high signal-to-noise ratio $\mathrm{P}$ wave, a near-zero amplitude (minimum energy) on the transverse component indicates a correctly aligned sensor if we assume isotropic material and no scattering along the ray path (Zietlow, 2016). A grid search was executed, with a $1^{\circ}$ increment from $0^{\circ}$ to $180^{\circ}$, to rotate the $\mathrm{HH} 1$ and $\mathrm{HH} 2$ seismograms to $\mathrm{HH}^{\prime}{ }^{\prime}$ and $\mathrm{HH} 2^{\prime}$. These 
values were used to identify the maximum amplitude ratio of the HH1'/HH2' components. The orientation that yielded the maximum ratio should be the radial component, and should be equal to the back azimuth of the event (with a possible ambiguity of $180^{\circ}$ ). Figure 2.2 shows an example of the horizontal components before and after finding the orientation at an event recorded by LOBS3.

\subsubsection{Surface wave orientation}

To determine orientations using surface waves, we adapted an algorithm developed for horizontal orientation estimation using the polarization of surface waves from teleseismic earthquakes in a previous OBS deployment in Cascadia (Stachnik et al., 2012). This algorithm was modified and an interactive routine was created to run in MATLAB with SAC formatted data (Lodewyk and Evers, 2014). We created a catalog composed of 31 teleseismic events with magnitudes greater or equal to Mw 6.0, distances ranging from $30^{\circ}-180^{\circ}$ from each station from various back azimuths and depths less than $100 \mathrm{~km}$ (Appendix A, Table A.1). For each station, the data were band-pass filtered from 0.02 to $0.04 \mathrm{~Hz}$ (50 to $25 \mathrm{~s}$ ), 10\% cosine tapered, rotated at 2 degree increments from $0^{\circ}$ to $360^{\circ}$ using an 800 second time envelope encompassing the predicted $4.0-\mathrm{km} / \mathrm{s}$ Rayleigh wave based on nominal group velocity, and the radial component $(\mathrm{HH} 1)$ was cross-correlated with the Hilbert transformed vertical component (HHZ). Rayleigh waves exhibit an elliptical particle motion observed on the radial and vertical components (e.g., Stein and Wysession, 2003). This retrograde ellipticity allows to resolve the $180^{\circ}$ ambiguity in orientation that is present in body wave methods (Stachnik et al., 2012). The Hilbert transform $90^{\circ}$ phase shift yields a linear relationship between the two signals. The vertical component and the Hilbert transformed radial component have a linear relationship (Stachnik et al., 2012) and a cross-correlation can be applied.

All events with a correlation value below 0.5 were automatically ranked as "bad". Each event with a correlation value above 0.5 was manually examined and ranked as "good", "bad", or "questionable" based on the visual similarity of the rotated radial and vertical seismograms. The horizontal component orientations were determined for each seismic event that was ranked as "good" and the final results were averaged and the uncertainty determined by taking the polar mean of the standard deviation for all "good" results at each station. An example of a waveform from an event with a high correlation and good signal-to-noise ratio, ranked 


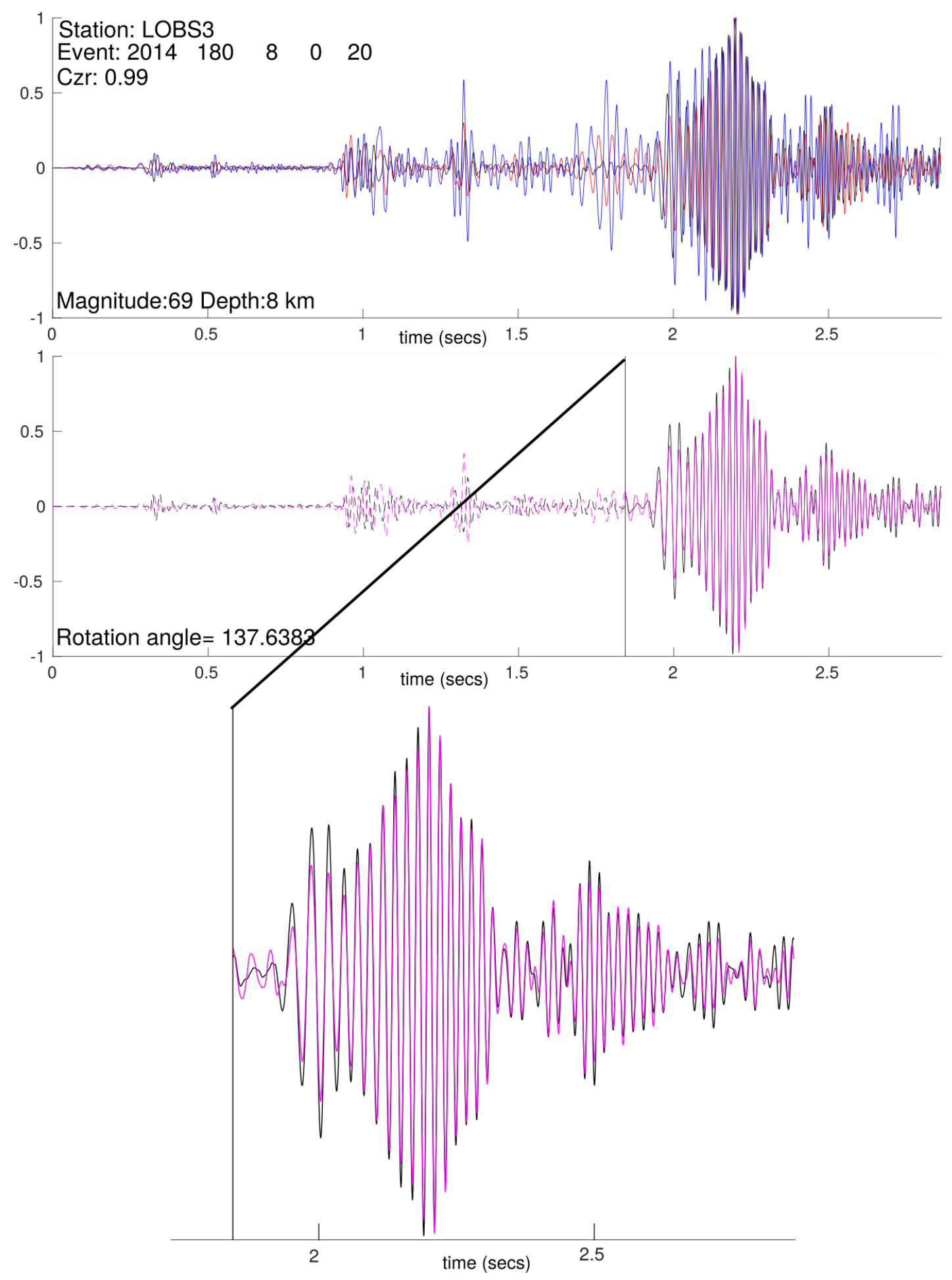

Figure 2.3: An example of an event from station LOBS3 characterized by high correlation and close to model waveform appearance, ranked as "good". The top waveform shows the filtered time series for HH1 (blue), HH2 (red), and HHZ (black). The bottom image displays the rotated radial (red) and vertical (black) components for the orientation angle that provides the highest correlation. The section of the waveform used for the orientation analysis is the surface wave to the right of the black vertical line. 
as "good" is shown Figure 2.3. Sub-plots have been generated for each station presenting orientation estimates versus back azimuths and correlation coefficients with mean value and uncertainty range (Appendix A, Figure A.1).

\subsubsection{Removal of poor seismic data}

Most recorded events were characterized by obscured $\mathrm{P}$ and surface wave arrivals due to long period noise (Stachnik et al., 2012). For the P wave method, we used only events with clear $\mathrm{P}$ wave arrivals that were unobscured by noise. For the Rayleigh wave study, the majority of low correlation (less than 0.5 ) values at each station were caused by high noise levels. The events with low correlation values were not used for horizontal orientation assessment. However, 'false estimates' can arise when the data are poor but channel correlations are nonetheless high. The code included procedures to recognize and reject these infrequent occurrences, which may occur for several reasons such as flat lined data or high noise levels. If the data was flat lined (has zeros as values) then the code assigned a non-number value and the seismograms were removed from further analysis. Events that yielded a high correlation value but were affected by high noise were manually examined and flagged as "bad" during the grading process and rejected.

\subsubsection{Horizontal orientation results}

Results from the determined sensor orientations of 6 LOBS and 5 EOBS stations are presented in Table 2.2. The resulting P-wave orientations compared well to Rayleigh-wave orientations determined using the Rayleigh wave method (Stachnik et al., 2012). To estimate an error in the orientation from the $\mathrm{P}$ wave analysis we took the standard deviation of all results for each station. Several events provided high correlations and good signal-to-noise ratios. Stations LOBS4 and LOBS5 provided no usable data. Although stations LOBS1, LOBS2 and LOBS10 ceased logging usable data in late 2014, we were able to orient stations LOBS1 and 10. LOBS6 did not provide consistent results between the two methods. All EOBS stations provided results using both methods. 
Table 2.1: Ocean-bottom orientation results.

\begin{tabular}{|c|c|c|c|c|c|c|c|c|c|c|c|c|c|}
\hline & & LOBS1 & LOBS3 & LOBS6 & LOBS7 & LOBS8 & LOBS9 & LOBS10 & EOBS1 & EOBS2 & EOBS3 & EOBS4 & EOBS5 \\
\hline & Lat $\left(^{\circ}\right)$ & -38.59 & -38.79 & -38.98 & -38.71 & -38.84 & -39.07 & -39.13 & -38.75 & -38.78 & -38.69 & -38.69 & -38.99 \\
\hline & Lon $\left(^{\circ}\right)$ & 178.82 & 179.15 & 178.8 & 178.57 & 178.46 & 178.52 & 178.31 & 178.68 & 178.58 & 178.65 & 178.82 & 178.33 \\
\hline & Depth (m) & -994 & -3540 & -1874 & -784 & -651 & -1457 & -1444 & -995 & -930 & -1023 & -1712 & -1348 \\
\hline \multirow{3}{*}{ P-wave } & orientation & 294.3 & 118.7 & 285.5 & 83.8 & 276.6 & 356.6 & 78 & 233.9 & 338.4 & 47.3 & 127.7 & 139.5 \\
\hline & \# meas. & 3 & 9 & 7 & 8 & 5 & 7 & 1 & 10 & 17 & 6 & 9 & 12 \\
\hline & STDEV & 19.9 & 2.7 & 12 & 6.6 & 13.6 & 5.5 & - & 7.2 & 15.5 & 25.2 & 16.4 & 12.1 \\
\hline \multirow{3}{*}{ R-wave } & orientation & 290 & 123.1 & 210.4 & 88.1 & 286.9 & 5 & 86.2 & 251.2 & 317.4 & 51.5 & 128.8 & 91.3 \\
\hline & \# meas. & 3 & 20 & 9 & 9 & 5 & 17 & 6 & 4 & 6 & 2 & 3 & 3 \\
\hline & STDEV & 8.4 & 8.7 & 33.4 & 9.4 & 10.8 & 6.3 & 7.9 & 4.2 & 7.4 & 5.6 & 2 & 7.4 \\
\hline P-R wave & Average & 292.2 & 120.9 & 248 & 85.9 & 281.7 & 0.8 & 82.1 & 242.5 & 327.9 & 49.4 & 128.2 & 115.4 \\
\hline
\end{tabular}

Note: "Orientation" refers to the Orientation of Channel 1 in degrees (relative to North). Depth of stations are in meters below sea level. 


\subsection{Shear wave splitting techniques}

The eigenvalue and transverse component minimization methods are two of the most commonly used methods to calculate shear wave splitting (SWS). The eigenvalue minimization method is commonly used in local earthquake splitting analysis, while the transverse component minimization method is most often used for teleseismic earthquake SKS splitting. We used the Multiple Filter Automatic Splitting Technique (MFAST; Savage et al., 2010b) to calculate SWS in Chapters 3 and 4 and the Splitracer software (Reiss and Rümpker, 2017) to calculate teleseismic SWS in Chapter 5. These two techniques are described in the following sections.

\subsubsection{MFAST: Automatic Shear Wave Splitting}

The local splitting in Chapter 3, 4 and 5 was analysed using the Multiple Filter Automatic Splitting Technique (MFAST; Savage et al., 2010b), a fully-automated shear wave splitting software, available at http:/ / mfast-package.geo.vuw.ac.nz/. This software was developed based on the eigenvalue minimization method (Silver and Chan, 1991) and the cluster analysis method (Teanby et al., 2004b). This method is especially suitable for the analysis of splitting measurements on large datasets of local earthquakes. Here we describe MFAST version 2.2.

MFAST requires the $S$ wave arrival time to first be determined. S-arrivals are handpicked in Chapter 3 and automatically picked in Chapter 4 and more information on these picking techniques can be found in the corresponding chapters.

Before calculating the SWS, MFAST first applies multiple band-pass filters on the $S$ arrival waveform and calculates the best signal-to-noise ratio (SNR). SWS parameters are determined for the best three filters out of a set of 14 predefined Butterworth filters with frequency band-widths within the range of $0.4 \mathrm{~Hz}$ to $10 \mathrm{~Hz}$.

Next, MFAST implements the SC91 SWS algorithm of Silver and Chan (1991), which performs a grid search over the fast azimuth and delay time parameter space and determines the best parameters that correct for splitting, measured by the minimum of the smallest eigenvalue of the corrected particle motion. This method iterates over all possible values of the fast azimuth, $\phi$, (in $1^{\circ}$ increments) and delay time, $\delta t$, (in $0.01 \mathrm{~s}$ increments, up to $1 \mathrm{~s}$ ) to calculate all possible splitting parameter solutions. Figure $2.4 \mathrm{~F}$ shows the contours of the smallest eigenvalue for 

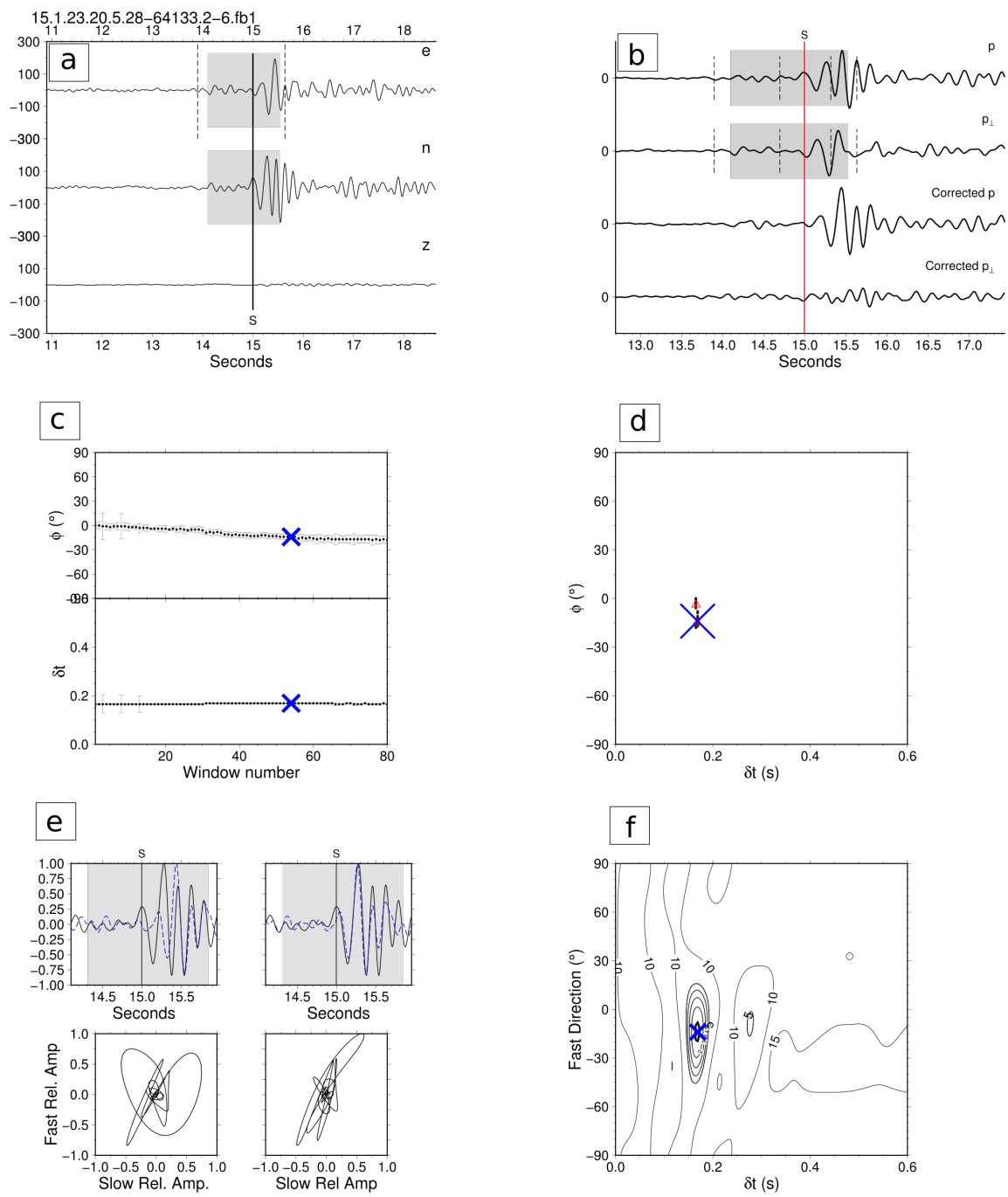

$$
\begin{aligned}
& \text { event 15.1.23.20.5.28-64133.2-6.fb } \\
& \text { depth: } 21.2005 \mathrm{~km} \\
& \text { distance: } 77.9805 \mathrm{~km} \\
& \text { magnitude: } 1.71 \\
& \text { - - - - - - - - - - - - - - - - - - - }
\end{aligned}
$$

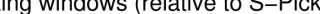

$$
\begin{aligned}
& \text { wbeg: }-1.10--0.30(5) \\
& \text { selected: } 14.096 \text { - 15.528, length: }
\end{aligned}
$$

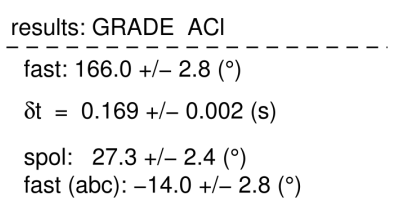

Figure 2.4: Example of an A grade shear wave splitting result for station LOBS8. (a) 3-10 Hz bandpass filtered components. Black line shows S-pick. Dashed lines show minimum start and maximum end time and grey shaded area is the window of the final measurement. (b) Normalized radial and transverse waveforms and radial and transverse corrected for splitting (c) $\Phi$ and $\delta t$ result for each window. The cross shows the final result. (d) Clustering of best results in $(\Phi, \delta t)$ space. The cross shows the final result. (e) Top graphs show the fast and slow waveform match. The bottom plots show the corresponding particle motions. (f) Clustering of best measurement with contour of the smallest eigenvalue of the covariance matrix for final result. The cross shows the final result. The bottom right label shows the final grade, $\Phi$, and $\delta t$ with their associated uncertainty. 

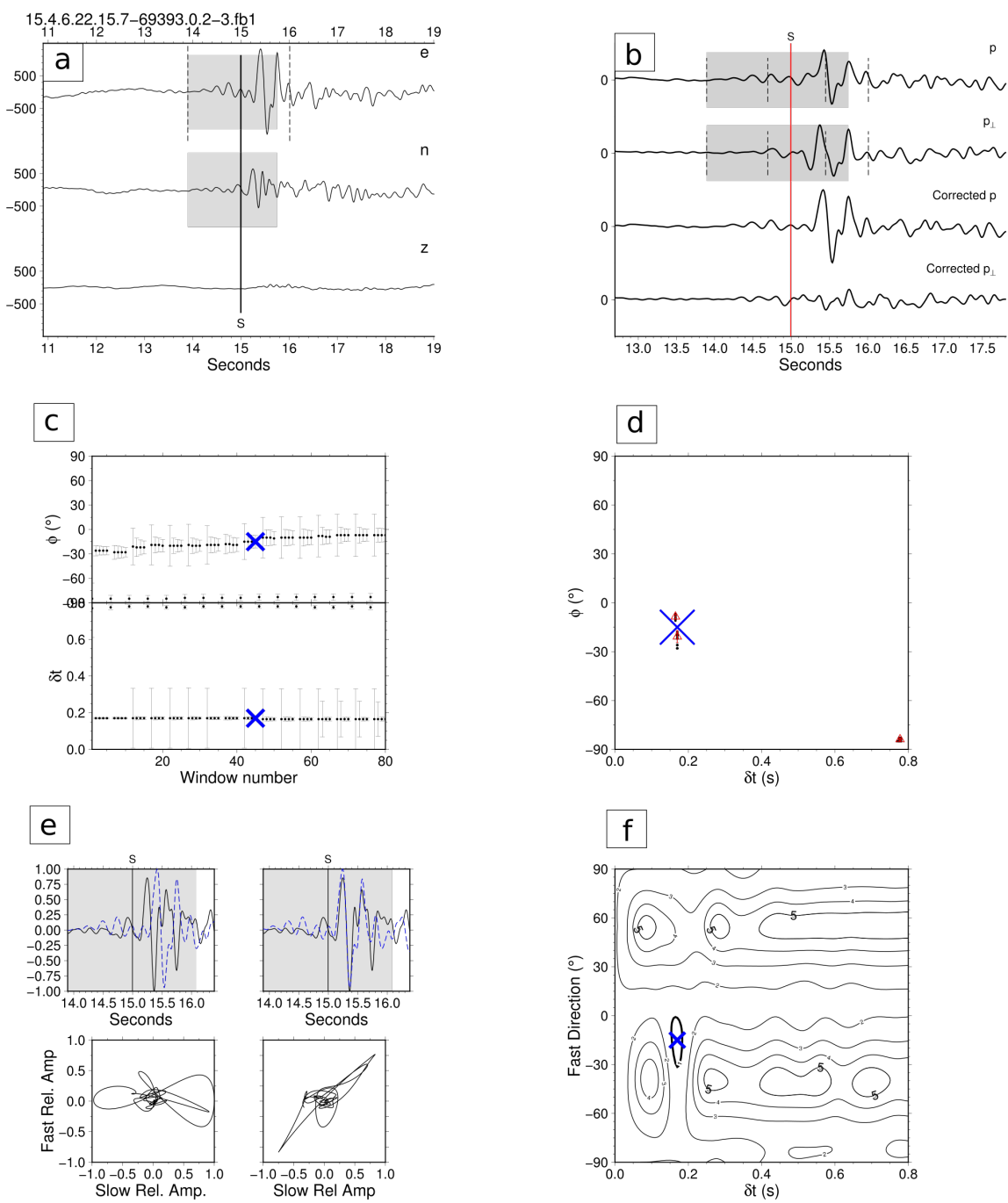

$$
\begin{aligned}
& \text { event 15.4.6.22.15.7-69393.0.2-3.ft } \\
& - \text { depth: } 17.4198 \mathrm{~km} \\
& \text { distance: } 67.9471 \mathrm{~km} \\
& \text { magnitude: } 2.5 \\
& -----------------1 \\
& \text { splitting windows (relative to S-Pick } \\
& \text { wbeg: }-1.10--0.30(5) \\
& \text { wend: } 0.45-1.01(16) \\
& \text { selected: } 13.896-15.749 \text {, length: } 1
\end{aligned}
$$

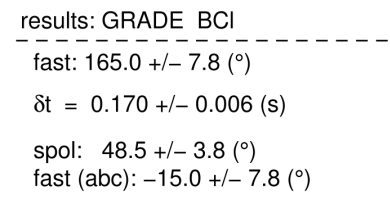

Figure 2.5: Example of a B grade shear wave splitting result for station LOBS8. (a) 3-10Hz bandpass filtered components. Black line shows S-pick. Dashed lines show minimum start and maximum end time and grey shaded area is the window of the final measurement. (b) Normalized radial and transverse waveforms and radial and transverse corrected for splitting (c) $\Phi$ and $\delta t$ result for each window. The cross shows the final result. (d) Clustering of best results in $(\Phi, \delta t)$ space. The cross shows the final result. (e) Top graphs show the fast and slow waveform match. The bottom plots show the corresponding particle motions. (f) Clustering of best measurement with contour of the smallest eigenvalue of the covariance matrix for final result. The cross shows the final result. The bottom right label shows the final grade, $\Phi$, and $\delta t$ with their associated uncertainty. 
a high grade measurement for the splitting parameters, $\phi$ and $\delta t$. This is equivalent to maximising the linearity of the particle motion (Figure 2.4E).

MFAST automatically analyses multiple measurement windows, whose length is based on the period of the wave, using the Silver and Chan (1991) method, and determines the best window using the cluster analysis technique of Teanby et al. (2004b). This technique searches for the most stable splitting parameters with the smallest errors, which are measured over several measurement windows. The parameters are scaled so that $\phi$ and $\delta t$ have the same weight. Each window represents a point on the scaled $\phi$ and $\delta t$ plane (Figure 2.4C). The hierarchical clustering is next applied based on the distance between the points. The resulting cluster with the lowest variance is chosen as the best cluster. The best $\phi$ and $\delta t$ measurement is the measurement from within that cluster with the smallest variance.

Finally MFAST assigns a grade from A to D, based on the SNR, uncertainty, distinctiveness of the cluster, and whether the measurement is a null (Savage et al., 2010b). Measurements with A and B grades are considered good quality splitting measurements and are analysed in Chapters 3,4 and 5. An example of an A and $B$ grade measurement calculated in Chapter 3 at station LOBS8 is shown in Figure 2.4 and 2.5, respectively. AB grade measurements are defined by the following criteria: the delay time is less than 0.8 times the maximum delay time for grid search, the SNR is greater than 3, the maximum error (one standard deviation) of the polarisation is less than $25^{\circ}$, and it is not a null measurement (Savage et al., 2010b).

The incidence angle for each event-station pair is determined using the Taup Toolkit (Crotwell et al., 1999) with a different 1-D velocity model used for each individual study area. Any rays with incidence angles greater than $35^{\circ}$ from vertical are excluded from the analyses as they are outside the shear wave window (Nuttli, 1961). Results with fast polarization $(\phi)$ within $20^{\circ}$ of the polarization of the incoming wave or its perpendicular are considered nulls (Peng and Ben-Zion, 2004) and are excluded from interpretation. Null results indicate that either the incoming wave was parallel or perpendicular to the fast azimuth, or there was no difference in the speeds of the split waves (Silver and Chan, 1991). The main steps associated with the MFAST are shown in Figure 2.6. 


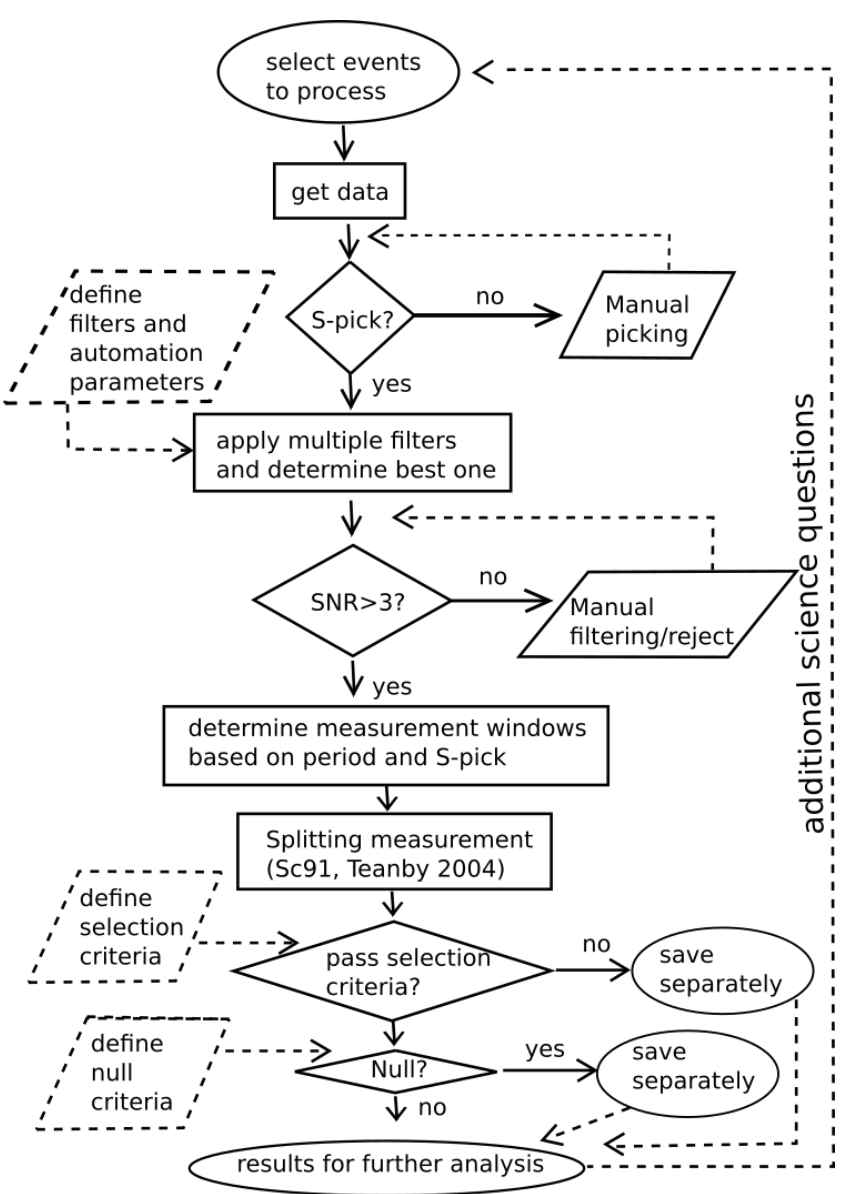

Figure 2.6: The processing steps of the Multiple Filter Automatic Splitting Technique (MFAST) [after Savage et al. (2010b)].

\section{Cycle Skipping}

Cycle skipping occurs when the fast and slow components are mismatched by an integer number of half-cycles, resulting in a shift in $\delta t$ which can provide either higher or lower $\delta t$ values and $90^{\circ}$ phase flips in $\phi$ compared to the correctly fit waveform. Most often, cycle skipping is a result of $S$ waves with narrow frequency ranges or by applying narrow band-pass filters. In order to avoid cycle skipping, the time windows chosen in MFAST include some of the signal before the $\mathrm{S}$ wave arrival so that the waves can be accurately matched.

\subsubsection{SplitRacer: Shear Wave Splitting}

Seismic waves from earthquakes at radial distances $>85^{\circ}$ travel through the core where, as a result of the P-to-S conversion at the core-mantle boundary (CMB), any record of down-going ray anisotropy is reset. The SKS and SKKS phase (or XKS 
phases) are the most commonly used phases to study upper mantle anisotropy, providing the best lateral resolution due to the steep angle of incidence of the phase arrivals. XKS phases of a shear wave are radially polarized from the coremantle boundary to the anisotropic source. If these up-going XKS phases encounter an anisotropic layer in the upper mantle, the two horizontal seismometer components will show lags in the arrival times of these phases. Thus, shear wave splitting of the upper mantle provides us with important information about the orientation and thickness of anisotropic material between the core-mantle boundary and the station.

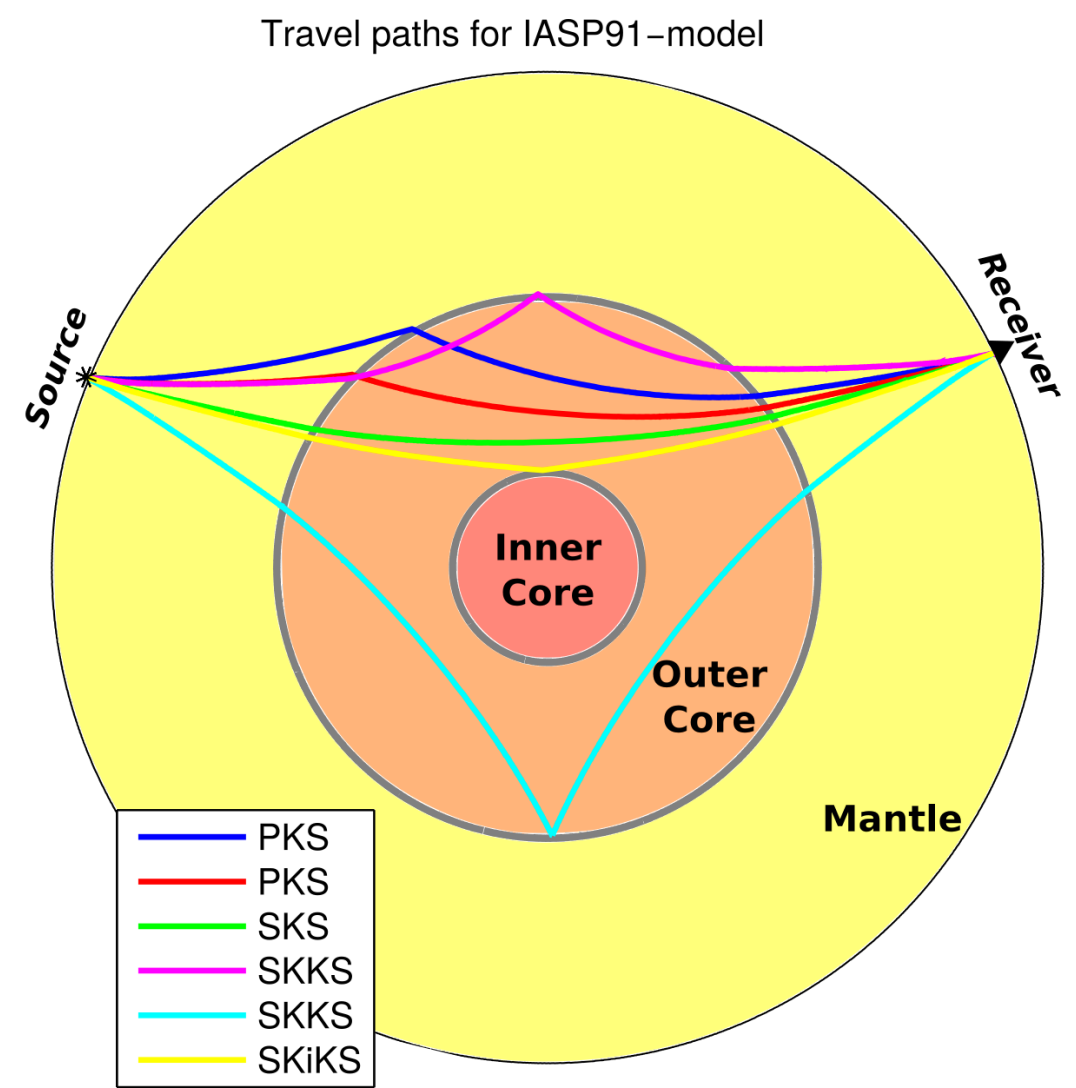

Figure 2.7: Travel paths for IASP91 model created using Taup (Crotwell et al., 1999) from a source at $0 \mathrm{~km}$ depth (star) to the receiver (triangle). Note path of XKS phases through the core-mantle boundary and the sharp incidence angle of these phases.

We use the SplitRacer software package by (Reiss and Rümpker, 2017), a semiautomatic processing approach which uses the minimum transverse energy technique of Silver and Chan (1991). The transverse component minimization method is the most widely used method for splitting measurements for broadband data (Silver and Chan, 1991). Here we describe the SplitRacer technique used to analyze tele- 
seismic SWS in Chapter 5.

Teleseismic earthquakes occurring between $85^{\circ}-180^{\circ}$ distance from each station are chosen for analysis. The chosen distance allows us to include all core phases, such as SKS, SKKS, SKIKS, PKIKS and PKS (called XKS phases; Figure 2.7).

The data are first bandpass filtered and a SNR of 1.5 is chosen to automatically select suitable phases of an event. XKS phases are found using the IASP91 velocity model in Taup (Crotwell et al., 1999). Selected phases are manually inspected during pre-processing and time windows are adjusted, if necessary.

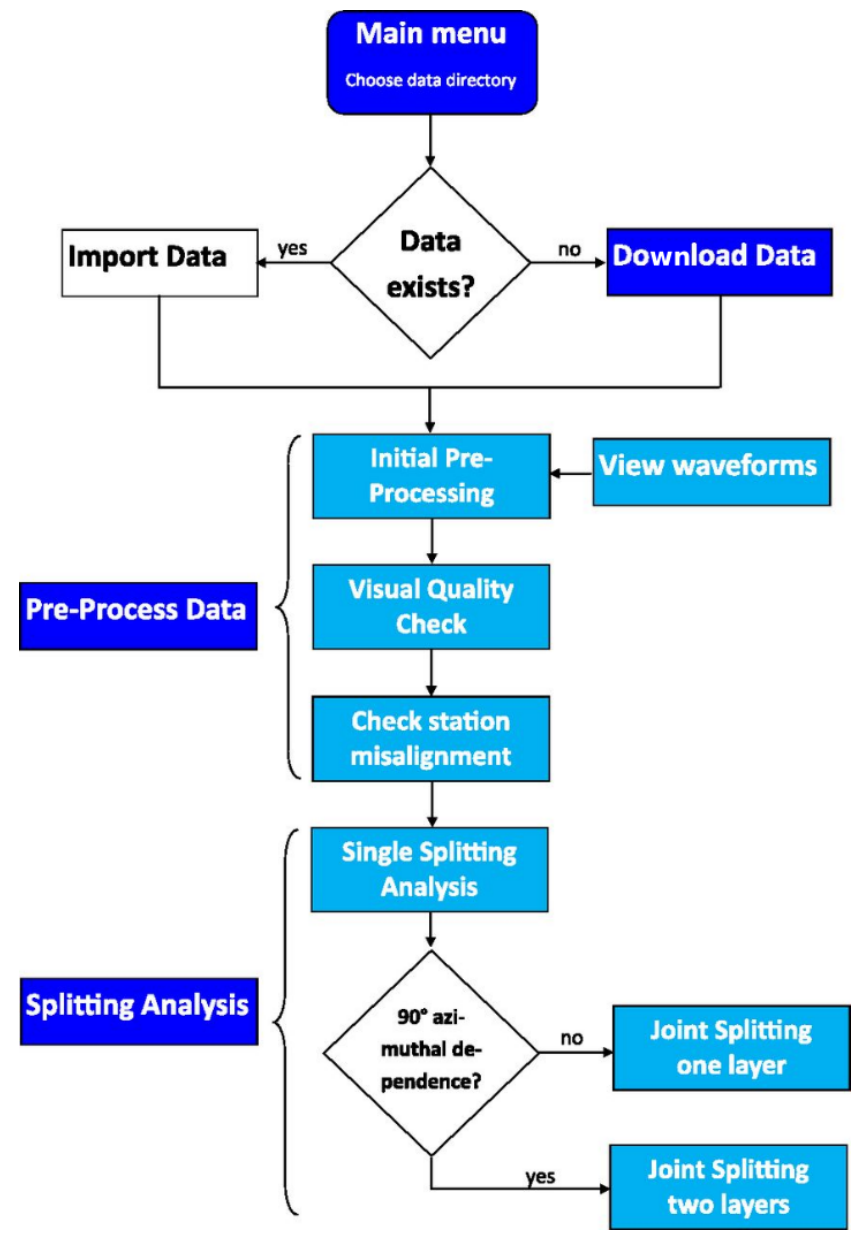

Figure 2.8: The processing steps of SplitRacer [after Reiss and Rümpker (2017)].

Once identified, the waveforms are rotated into a coordinate frame of radial and transverse polarization. The minimum transverse energy technique uses a grid search approach over all $\phi$ and $\delta t$ values that best minimize the energy on the transverse component (Silver and Chan, 1991). This method iterates over all possible values of the fast azimuth, $\phi$, (in $1^{\circ}$ increments) and delay time, $\delta t$, (in 0.1 
s increments) to calculate all possible splitting parameter solutions. If energy is present on the transverse component and we observe an elliptical particle motion it indicates the possible presence of anisotropy from the CMB to the receiver (Silver and Chan, 1991). The absence of energy on the transverse component indicates either a lack of anisotropic material along the ray path, termed null, or less likely that the backazimuth of the ray is parallel to the fast or slow direction (Silver and Chan, 1991). We use the predefined 50 randomly-chosen different time windows for analysis, allowing for a thorough statistical analysis of the results. The correction of the particle motion is manually checked for each measurement. Each measurement is marked as either "good", "average", "bad", or "null" and all but "bad" measurements are used for further analyses in Chapter 5.

In addition to the single phase splitting measurements, a single layer inversion is applied by using all waveforms characterized as either "good", "average" or "null". The inversion for a single layer stacks the single phase splitting measurements, which yields a single $\Phi$ and dt value that best reduces the transverse energy on all phases simultaneously for each station, similar to the approach by Wolfe and Silver (1998). This inversion allows for the reduction of noise and increases the robustness of the measurements (Reiss and Rümpker, 2017). The main steps associated with SplitRacer are shown in Figure 2.8. 


\section{Chapter 3}

\section{Temporal and spatial variations in seismic anisotropy and $V_{P} / V_{S}$ ratios in a region of slow slip}

Zal, H. J., Jacobs, K., Savage, M. K., Yarce, J., Mroczek, S., Graham, K., Todd,E. K., Nakai, J., Iwasaki, Y., Sheehan, A., Mochizuki, K., Wallace, L., Schwartz, S., Webb, S., and Henrys, S. (2020). Temporal and spatial variations in seismic anisotropy and $V_{P} / V_{S}$ ratios in a region of slow slip. Earth and Planetary Science Letters, 532:115970

\subsection{Abstract}

In September 2014, a five week long slow slip event (SSE) occurred near Gisborne at the northern Hikurangi subduction zone, New Zealand, and was recorded by offshore instruments deployed by the Hikurangi Ocean Bottom Investigation of Tremor and Slow Slip (HOBITSS) project. Up to $25 \mathrm{~cm}$ of slip occurred directly below the HOBITSS array. We calculate shear wave splitting (SWS) and $V_{P} / V_{S}$ ratios for event-station pairs on HOBITSS ocean bottom seismometers and onshore GeoNet seismic stations to determine the relationship in time and space between slow slip and these seismic properties. Spatial averaging of SWS fast azimuths yields trench-perpendicular fast azimuths in some areas, suggesting that compressive stress from plate convergence closes microcracks and controls anisotropy in the upper-plate. Variations from the trench perpendicular directions are observed near a subducting seamount, with directions closely resembling fracture and fault 
patterns created by subducting seamounts previously observed in both laboratory and field experiments. Temporal variations in fast azimuths are observed at three stations, two of which are located above the seamount, suggesting measurable variations in stress orientations. During the SSE, median $V_{P} / V_{S}$ measurements across all offshore stations increase from 1.817 to 1.894 and SWS delay times decrease from $0.178 \mathrm{~s}$ to $0.139 \mathrm{~s}$ (both changes are significant within $95 \%$ confidence intervals). Temporal variations in $V_{P} / V_{S}$ and delay time are consistent with fluid pressurization below a permeability barrier and movement of fluids during the rupture of a slow-slip patch.

\subsection{Introduction}

Slow slip events (SSEs) are characterized as slow, earthquake-like motions that release energy over periods ranging from days to years. Understanding the physical processes occurring during SSEs is particularly important from an earthquake hazard perspective, as SSEs may influence the timing of nearby large earthquakes (e.g., Obara and Kato, 2016), or alternatively be triggered by earthquakes (e.g., Wallace et al., 2018). The driving mechanisms behind the occurrence and nucleation of SSEs are still poorly understood, but observational and modelling studies suggest that SSEs arise in the transition zone between velocity strengthening (i.e., aseismic creep) and velocity weakening (i.e., stick slip) behavior on the plate interface, possibly in the presence of high fluid pressures (e.g., Audet et al., 2010).

From May 2014 to June 2015, the Hikurangi Ocean Bottom Investigation of Tremor and Slow Slip (HOBITSS) project deployed 15 Ocean Bottom Seismometers (OBS) and 24 Absolute Pressure Gauges (APG) with the goal of recording offshore seismological and geodetic data during an SSE (Wallace et al., 2016). From late September to late October 2014 an SSE occurred offshore Gisborne, directly below the HOBITSS array (Figure 3.1; Wallace et al., 2016). The September-October 2014 Gisborne SSE is the first in New Zealand to be recorded by offshore instruments.

Here, we use local earthquake data from nine HOBITSS OBSs and nine GeoNet permanent land-based seismic stations to determine crustal anisotropy, where most S-phases travel directly from the source to receiver. As a shear wave enters a seismically anisotropic medium it is split into two orthogonally polarized quasi-shear waves with a fast and slow polarization (Crampin, 1981). The anisotropy is 


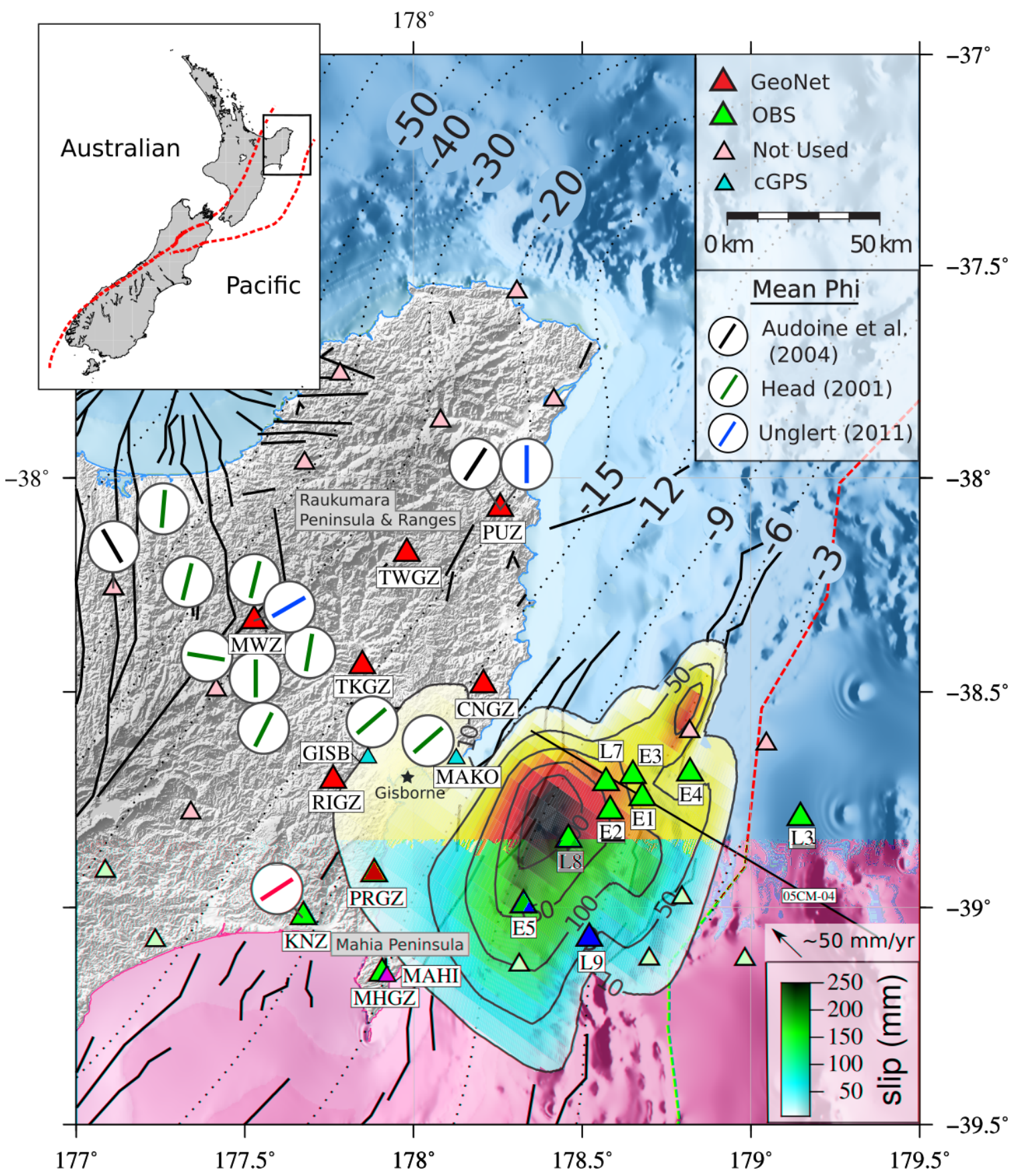

Figure 3.1: Locations of onshore Geonet (http://www.geonet.org.nz) seismic stations (red triangles) and HOBITSS ocean bottom seismometers (green triangles) off the coast of Gisborne (EOBS, short period ocean bottom seismometers; LOBS, broadband ocean bottom seismometers). Station names have been shortened from LOBS and EOBS to L and E. Pink triangles represent seismic stations not used in this study. Blue triangles are GeoNet cGPS stations. Mean fast azimuths from previous studies have been plotted inside white circles. Slip contours (in $\mathrm{mm}$ ) for the SeptemberOctober 2014 SSE (SSE2) are from Wallace et al. (2016); dotted contours of plate interface (in $\mathrm{km}$ ) are from Williams et al. (2013); dashed red line of plate boundary interface is from Coffin et al. (1998); black line marked 05CM-04 is a 2-D seismic profile (Barker et al., 2009); solid black lines of active faults are from Litchfield et al. (2014). 
represented by two splitting parameters; $\Phi$, the polarization of the fast azimuth (or "fast azimuth") of the anisotropic material and $\delta t$, the delay time accrued during the travel through the anisotropic material. The delay time is dependent on the path length within the anisotropic material and the difference between the velocity of the fast and slow polarized waves. If anisotropy changes along the path, the splitting will be most sensitive to the last layer traversed (e.g., Rumpker and Silver, 1998).

In the crust, anisotropy is mainly caused by either pre-existing structural features, such as faults and dikes, or by the preferential closing of microcracks at particular orientations as a response to horizontal differential stresses (Babuska and Cara, 1991). In the upper crust, fast azimuths tend to be parallel to fluid filled microcracks and to the maximum principal stress (Crampin, 1981). Differential horizontal stress will preferentially close the cracks that are oriented orthogonal to the maximum horizontal compressive stress $\left(S_{H} \max\right.$; Nur, 1971). Cracks tend to close completely with depth due to lithostatic pressure. Therefore, most studies of upper crustal anisotropy find little change in splitting with depth, suggesting that the top few kilometers are most important for splitting measurements (e.g., Savage et al., 2010b). Cracks are also important in controlling the ratio of the speeds of seismic compressional and shear waves, $V_{P} / V_{S}$. With higher quantities of liquidfilled cracks, $\mathrm{S}$-wave velocities are reduced more than $\mathrm{P}$ waves and hence the ratio increases (Nur, 1971). In this paper we use the complementary sensitivity of $V_{P} / V_{S}$ and SWS measurements to cracks in the upper crust to evaluate the stress field before, during and after the September-October 2014 SSE.

\subsubsection{Hikurangi Subduction Zone and the September-October 2014 SSE}

The Pacific and Australian plates converge through the North Island region at $~ 50$ $\mathrm{mm} / \mathrm{yr}$, at an oblique angle to the orientation of the plate boundary (Figure 3.1; e.g., Wallace et al., 2004). Slip is partitioned with the trench perpendicular component accommodated on the subduction thrust, and a trench parallel component largely accommodated by upper-plate strike-slip faulting and clockwise rotation of the North Island forearc (Beanland and Haines, 1998; Wallace et al., 2004). North Island's main geological features, such as the Raukumara mountain ranges and 
the North Island Dextral Fault Belt, tend to follow a trench parallel, NNE-SSW, direction (Beanland and Haines, 1998). Focal mechanisms from the Raukumara Peninsula region show a maximum compressive stress that is trench parallel in the overriding plate (Townend et al., 2012).

Since 2002, more than 30 SSEs have been documented and observed in New Zealand using continuous Global Positioning System data (cGPS; e.g., Wallace and EberhartPhillips, 2013; Wallace et al., 2016). These events vary in duration, from six days to 1.5 years, with equivalent moment release from $\mathrm{Mw}$ 6.3-7.2, depths from 2-60 km, and recurrence intervals of 2 to 5 years (e.g., Wallace and Beavan, 2010). The SSEs occurring offshore the East Coast of the northern Hikurangi trench are of particular interest due to their shallow depth (less than $15 \mathrm{~km}$ ), shorter recurrence intervals ( $<2$ years), short duration (a few weeks or less) and large horizontal deformation signals (1-3 cm) on onshore stations (e.g., Wallace and Beavan, 2010; Wallace et al., 2016).

A total of four SSEs (SSE1-SSE4) occurred within close proximity to the HOBITSS array during the deployment; the timing of SSE1-4 were calculated from timedependent geodetic inversions by Warren-Smith et al. (2019). SSE1, the earliest and longest, occurred south of the array, between September and November 2014. The September-October 2014 SSE (SSE2) started 20 days after the start of SSE1, directly beneath the array and was the largest and best recorded SSE; it was recorded on APGs as well as onshore permanent cGPS stations (Figure 3.1; Wallace et al., 2016). We emphasize the main pulse of SSE2, between Julian days 265-285. Tremor and repeating earthquakes began a few days before day 285 (Todd et al., 2018; Shaddox and Schwartz, 2019). SSE2 tails off between days 286-300 (Warren-Smith et al., 2019), so that the extended period of SSE2 is five weeks long; the tremor and repeating earthquakes continue throughout the tail-off period. The vertical deformation resolved from the HOBITSS APGs during SSE2 enabled better delineation of the trench-ward extent of SSEs and suggests that slip occurred to within $2 \mathrm{~km}$ of the seafloor, and perhaps all the way to the trench (Wallace et al., 2016). SSE3 occurred in December 2014 just southwest of the array and SSE4 occurred in February 2015, also southwest of the array. 


\subsubsection{Previous results}

\section{Shear Wave Splitting results}

Previous SWS analyses across the Raukumara Peninsula using local earthquakes have shown mainly trench-parallel fast azimuths, ranging from $\sim 0^{\circ}$ to $30^{\circ}$, on the eastern side of the Raukumara Peninsula, and larger variations in azimuths further west (Figure 3.1; Head, 2001; Audoine et al., 2004; Unglert, 2011). Our dataset complements that of previous studies and extends crustal splitting results offshore for the first time.

\section{$\mathbf{V}_{\mathbf{P}} / \mathbf{V}_{\mathbf{S}}$}

Typically, $V_{P} / V_{S}$ ratios are thought to increase when fluid pressures approach lithostatic, or in areas with high clay content (e.g., Eberhart-Phillips et al., 2017). Tomographic studies have shown that regions of slow-slip have elevated fluid pressures and tend to have high Poisson's ratios and high $V_{P} / V_{S}$ ratios (e.g., Audet et al., 2010). Seismic attenuation (Eberhart-Phillips et al., 2017) and residual traveltimes (Bassett et al., 2014) are both higher in the Northern Hikurangi trench than they are further south for similar depths. This is consistent with a more fluid-rich system in the north. The source area of a deep SSE occurring in Central Hikurangi, $\sim 100 \mathrm{~km}$ southwest of the September-October 2014 SSE, coincides with a high $V_{P} / V_{S}$ area, ranging from $\sim 1.7-1.8$, and is interpreted as a fluid-rich and highly over-pressurized region (Wallace and Eberhart-Phillips, 2013). The subduction interface is located at $\sim 15 \mathrm{~km}$ beneath the Gisborne area, with a westerly $10^{\circ}$ dip (Williams et al., 2013). Subducting seamounts are imaged between the East Coast of the Raukumara Peninsula and the Hikurangi trench (Figure 3.1; Bell et al., 2010), and many more seamounts protrude above the sedimentary cover on the not-yet subducted Pacific Plate. Zones of high-amplitude interface reflectivity are associated with these subducting seamounts and have been interpreted as entrained fluid-rich sediments that may promote slow slip (Bell et al., 2010). These high-amplitude reflectivity regions of the plate interface are associated with high $V_{P} / V_{S}(\sim 1.8)$ and low seismic attenuation ratios (Qs/Qp $<1$; Eberhart-Phillips et al., 2017).

Temporal and spatial variations in $V_{P} / V_{S}$ have been observed during large earth- 
quakes and SSEs. Husen and Kissling (2001) found an increase in $V_{P} / V_{S}$ above a suggested permeability barrier following the 1995, 8.0 Mw, Antofagasta Earthquake on the plate interface in Chile. They proposed that a permeability barrier allows lithostatic pore pressures to build along the seismogenic zone while hydrostatic pore pressures remain in the overlying crust. Furthermore, they suggest that only large earthquakes, such as the Mw 8 Antofagasta earthquake, can break these permeability barriers on a scale large enough to induce enough fluid flow to change $V_{P} / V_{S}$ observations. However, observations by Nakajima and Uchida (2018) in Kanto Japan show cyclic variations in seismic attenuation and seismicity rates with the occurrence of SSEs and conclude that such permeability barriers may also break during SSEs. Recently, Warren-Smith et al. (2019) helped support this model; they used focal mechanisms from the HOBITSS OBS dataset to show that both crustal stress and fluid pressure temporally evolve through SSE cycles, suggesting that recurring breaches of permeability barriers also occur in the Hikurangi subduction zone in regions of shallow slow slip.

\subsection{Data}

The HOBITSS OBSs consisted of 10 Lamont-Doherty Ocean Bottom Seismograph Instrument Pool (OBSIP) broadband OBS (LOBS) and five University of Tokyo (Earthquake Research Institute) short period OBS (EOBS; Wallace et al., 2016). The array was deployed offshore Gisborne from May 2014 to June 2015 and complemented the onshore GeoNet network (Figure 3.1). We have calculated the horizontal orientations of the HOBITSS OBS receivers using both a P-wave (Zietlow, 2016) and Rayleigh wave (Stachnik et al., 2012) method (Chapter 2). The earthquakes used for orientation analyses are presented in Appendix A (Table A.1) and our orientation results are presented in Chapter 2 (Table 2.2) and provide the basis for further analyses using all three components in the HOBITSS project. LOBS1, 2, 4, 5,6 , and 10 had instrument problems and/or did not acquire enough data for instrument orientation and are omitted from further analysis in this study. Using the HOBITSS earthquake catalog of Yarce et al. (2019), with our depth constraint $(<50$ $\mathrm{km}$ depth), yields 2,140 earthquakes with magnitudes ranging from ML 0-4.73 (Figure 3.2). The median depth of the earthquakes is $23 \mathrm{~km}$, with most earthquakes occurring in the subducting plate. We manually picked an additional 14,311 $\mathrm{P}$ and S arrivals at offshore stations EOBS1-5 and LOBS3, 7, 8, and 9. We combined these 
picks with 32,394 P and S arrivals from Yarce et al. (2019) on the same nine offshore stations and nine onshore GeoNet stations (CNGZ, KNZ, MHGZ, MWZ, PRGZ, PUZ, RIGZ, TKGZ, TWGZ), resulting in 15,072 P-S phase pairs, where both $\mathrm{P}$ and $\mathrm{S}$ arrivals were present on the same seismogram.

Stations LOBS3, 7 and 9 experienced timing issues. These timing issues do not affect SWS results but could alter $V_{P} / V_{S}$ results. To test for systematic affects from these stations we compared the $V_{P} / V_{S}$ results for stations with and without timing errors (Appendix B, Section B.1, Figure B.1). We find only minor differences in these two subsets, so these stations are included in the analyses.

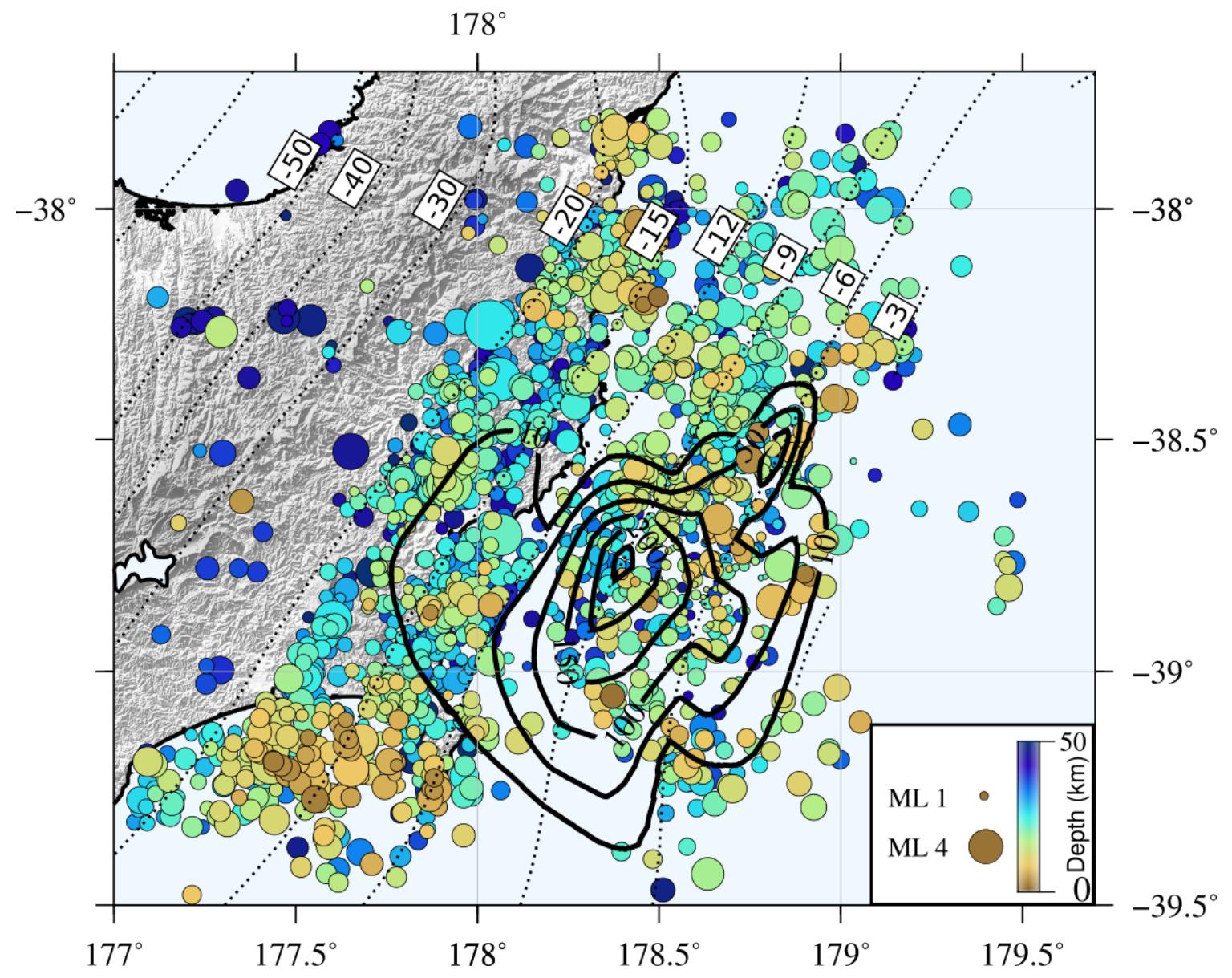

Figure 3.2: Earthquake locations in our study area, and above $50 \mathrm{~km}$ depth, selected from the HOBITSS catalog (Yarce et al., 2019). The catalog runs between May 2014 to June 2015 and earthquake magnitudes (ML) range from 0 to 4.73. Slip contours for SSE2 (in mm) are from Wallace et al. (2016); dotted contours of plate interface (in km) are from Williams et al. (2013). 


\subsection{Methods}

\subsubsection{Automatic Shear Wave Splitting (MFAST)}

The splitting was analysed using MFAST (Multiple Filter Automatic Splitting Technique; Savage et al., 2010b). MFASTv2.2 executes the following steps: (1) apply multiple band-pass filters on the $S$ arrival waveform and calculate the best product of signal-to-noise ratio (SNR) and bandwidth; (2) apply the SWS algorithm of Silver and Chan (1991) to perform a grid search over the fast azimuth and delay time parameter space and determine the best parameters that correct for splitting, measured by the minimum of the smallest eigenvalue of the corrected particle motion; (3) automatically analyse multiple measurement windows, whose length is based on the period of the wave and whose starting values depend on the $S$ arrival, using the Silver and Chan (1991) methods, and determine the best window using the cluster analysis technique of Teanby et al. (2004b); (4) assign a grade from A to $\mathrm{D}$, based on the SNR, uncertainty, distinctiveness of the cluster, and whether the measurement is a null (Savage et al., 2010b).

The incidence angle for each event-station pair was determined using the Taup Toolkit (Crotwell et al., 1999) with a 1-D velocity model (Appendix B, Table B.1) created for the study area from the 3-D model by Eberhart-Phillips et al. (2010). Because rays with incidence angles greater than 35 degrees from vertical are outside the shear wave window, they were excluded from the analyses (Nuttli, 1961). Delay times typically show more scatter compared to fast azimuths (e.g., Gao and Crampin, 2006). We used both A and B grade measurements for our analyses (examples are shown in Chapter 2, Figures 2.4 and 2.5).

\subsection{2 $\quad \mathrm{V}_{\mathrm{P}} / \mathrm{V}_{\mathrm{S}}$}

We calculated the ratio of P- to S-velocities at each station for all 15,072 P-S phase pairs (10,035 offshore, 5,037 onshore). Not limited to A and B grade measurement criteria, the quantity of phase pairs was larger than that for SWS analysis. Following the approach of Wadati and Oki (1933) we calculated an average ratio of P- to S-velocity $\left(V_{P} / V_{S}\right)$ along the ray path from the arrival times at each station:

$$
V_{P} / V_{S}=t_{S} / t_{P}
$$


where $t_{S}=T_{S}-T_{O}$ and $t_{P}=T_{P}-T_{O}$, with $T_{S}$ and $T_{P}$ being the arrival times of the $S$ - and $\mathrm{P}$ - wave, respectively, and $T_{O}$ is the origin time of the earthquake.

\subsubsection{Moving averages and $95 \%$ confidence intervals}

To look for temporal changes at each station, we determined the moving median values of all $V_{P} / V_{S}$ and SWS delay time measurements. We calculated the $95 \%$ confidence intervals (CIs) of the median using the method of Altman et al. (2000) (Appendix B, Section B.2). Generally, the median is more robust than the mean, and less sensitive to outliers, but is difficult to apply to azimuthal values. Therefore, temporal analysis of fast azimuths uses circular means with confidence intervals determined by bootstrapping (Appendix B, Section B.2).

We chose a 20-day window, with a window step of 1 day, after examining several window lengths. The 20-day time window was narrow enough to reveal changes within the 35 day-long SSE2 without excessive smoothing and wide enough to provide acceptable confidence intervals. The 20-day moving windows are plotted at the center of the window, so any observed changes could start 10 days earlier or later.

\subsubsection{Spatial averaging}

To calculate spatial averages of fast azimuths, $(\Phi)$, we used the Tomography Estimation and Shear-wave-splitting Spatial Average (TESSA) package by Johnson et al. (2011) over a spatial area focused on the SSE region and using a subset of 1,893 earthquakes. TESSA works by gridding the area and then assigning the fast azimuths for each earthquake to every spatial grid-block through which the ray passed. To account for potential overprinting of $\Phi$ later in the path, the individual $\Phi$ values within each grid-block are weighted inversely proportional to the square of the distance from the station. We assigned a minimum block size of $3 \mathrm{~km}$ which resulted in 10-65 rays passing through each grid-block (Appendix B, Figure B.2). Fast azimuths were not plotted for blocks with standard deviation of the average greater than $30^{\circ}$ and standard error larger than $10^{\circ}$ and for areas with sparse raypath coverage (mainly in the eastern study area; Appendix B, Figure B.2). 


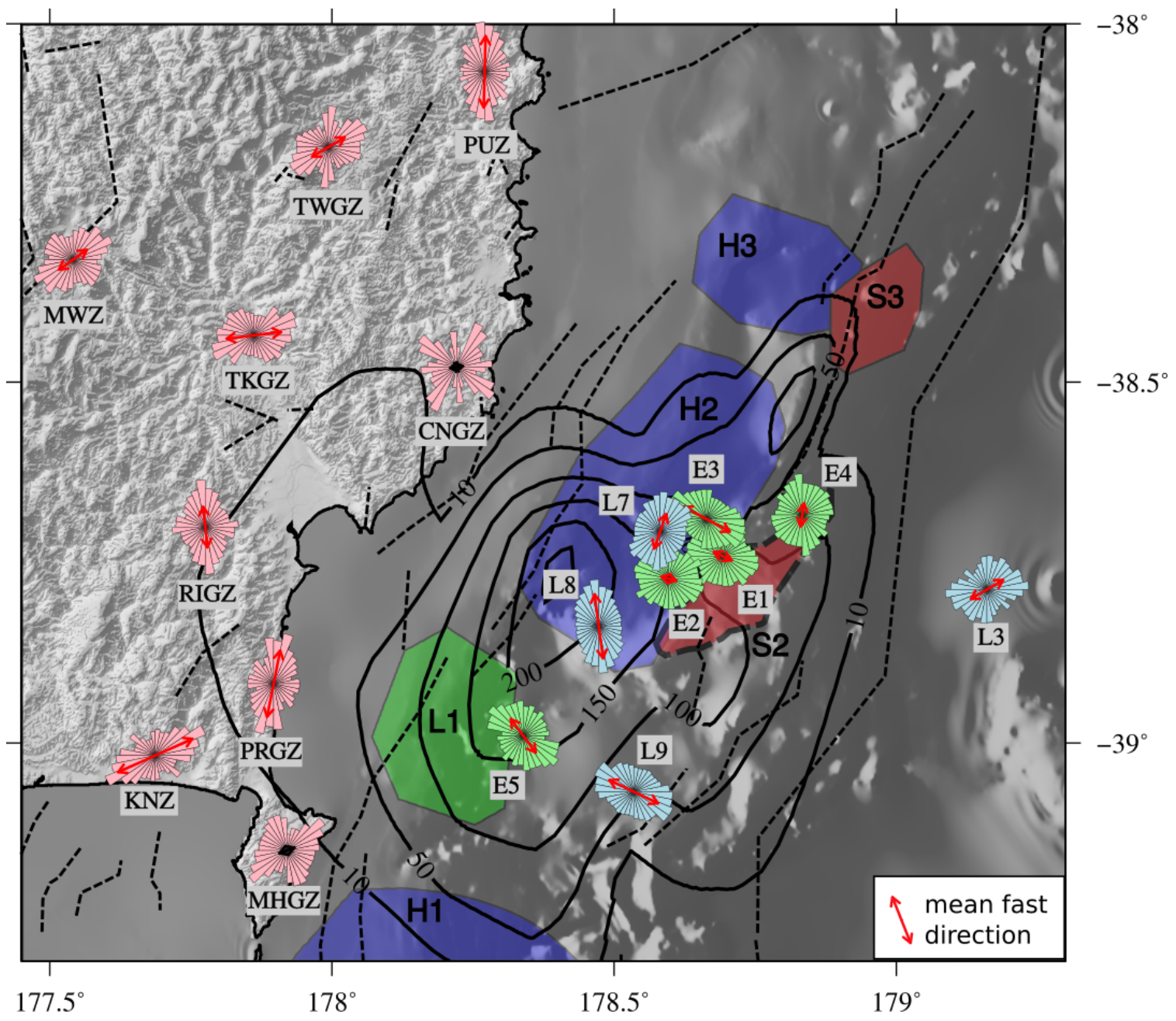

Figure 3.3: Rose diagrams of fast azimuths calculated using MFAST (Savage et al., 2010b). Rose diagrams are plotted on the stations at which measurements were made. The lengths of the sectors are proportional to the square root of the number of measurements in each sector. Red arrows are the means and are scaled by their resultant length. S2 and S3 indicate areas of seamounts. H1, $H 2$, and $H 3$ indicate high-amplitude reflectivity zones and $L 1$ is a lens reflectivity zone (Bell et al., 2010), with updated S2 boundary from Barker et al. (2018). Slip contours (in mm) for SSE2 are from Wallace et al. (2016). Dashed black lines mark active faults from Litchfield et al. (2014).

\subsection{Results}

\subsubsection{Fast azimuths and spatial averaging}

A total of 10,676 splitting measurements (8,333 offshore and 2,343 onshore) yielded A and B grades. Figure 3.3 shows rose diagrams of the fast azimuths plotted at the station at which measurements were made. Mean fast azimuths vary across both onshore and offshore stations. To distinguish stations with significant mean 
fast azimuths from those with uniformly distributed fast azimuths, we used a Rayleigh test for circular uniformity (Rayleigh test p-value $<0.05$ shows a significant mean fast azimuth). All stations, except CNGZ and MHGZ, have significant mean azimuths with more than 100 measurements (Table 3.1). Therefore, CNGZ and MHGZ were excluded from the station average interpretation.

We observe complexity in the rose diagrams and in the spatially averaged fast azimuths (Figures 3.3 and 3.4). A trench-perpendicular NW-SE mean $\Phi$ azimuth is observed in the southern offshore region, across stations LOBS9 and EOBS5. The NW-SE trend is disrupted near the western, downdip, portion of seamount $S 2$ and across the related high-amplitude reflectivity zone $H 2$, similar to changes observed in mean fast azimuths at stations EOBS1, 2, 3 and LOBS7 in Figure 3.3. Onshore stations PRGZ, RIGZ, and PUZ show a N-S trending mean azimuth. Stations MWZ and TWGZ have a NE-SW azimuth, station KNZ has a ENE-WSW azimuth and TKGZ has an azimuth closer to E-W.

\subsubsection{Temporal changes in fast azimuths, delay time and $V_{P} / V_{S}$ measurements}

Figure 3.5 shows $V_{P} / V_{S}$, $\delta t$, and $\Phi$ for individual offshore stations as a function of time. Stations LOBS9 and EOBS1 and 4 experience large changes in $\Phi$ but their calculated $95 \%$ confidence intervals are large and span $\sim 180^{\circ}$ at certain times. Station LOBS9 exhibits a $\sim 180^{\circ}$ change in $\Phi$ before SSE2, with $\Phi$ rotating from $\sim-70^{\circ}$ (NWSE) to $\sim 90^{\circ}(\mathrm{E}-\mathrm{W})$ between Julian days 240 and 270 . LOBS9 is located south of seamount $S 2$, on a bend in the slip contours. Stations EOBS1 and EOBS4 exhibit a $\sim 90^{\circ}$ rotation in $\Phi$ during SSE2, between Julian days 255-310. EOBS1 rotates from $\sim 135^{\circ}$ (NW-SE) to $\sim 30^{\circ}$ (NE-SW). EOBS4 rotates from $\sim 0^{\circ}(\mathrm{N})$ to $\sim-70^{\circ}$ (NW-SE). Both stations EOBS1 and EOBS4 are located between seamount $S 2$ and associated high-amplitude reflectivity zone $H 2$. $\Phi$ at stations LOBS3, 7, 8 and EOBS2, 3 and 5 are generally more stable with time.

An increase in $V_{P} / V_{S}$ occurs at all offshore stations during the main slip pulse of SSE2 (Figure 3.5). A decrease in delay times occurs during SSE2 at stations EOBS2, 3, 4, and 5, and LOBS3. Station EOBS1 and LOBS7, 8 and 9 exhibit relatively stable delay times during this period. Thus, at five stations, we observe both a decrease in delay time and increase in $V_{P} / V_{S}$ during SSE2. 


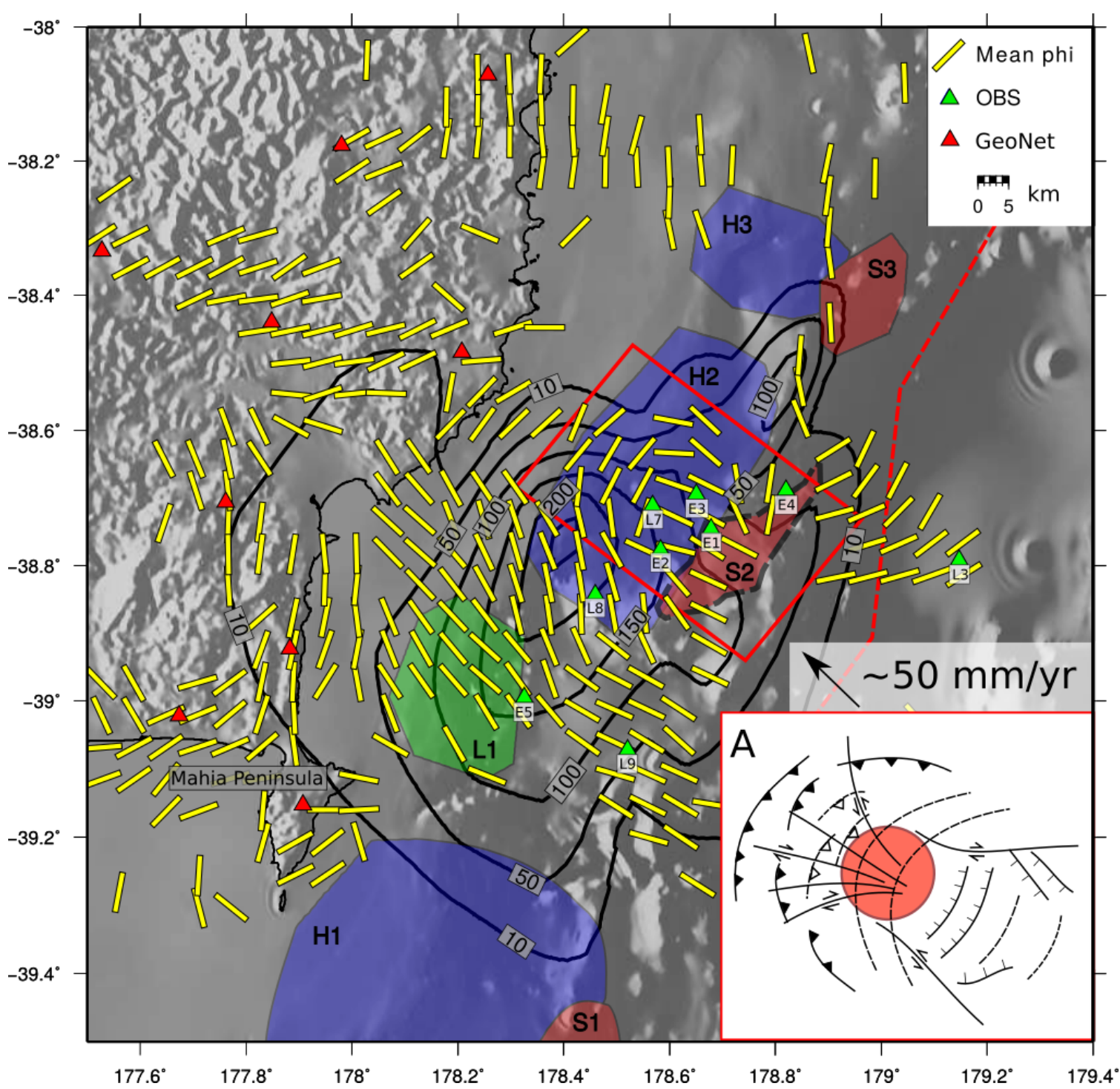

Figure 3.4: Spatial averages of SWS fast azimuths from TESSA (Johnson et al., 2011) using the 1,893 earthquakes that are within the boundaries shown here. Yellow bars show average fast azimuths for each block plotted in the center of the grid block. Fast azimuths are not plotted for blocks with average values that have a standard deviation greater than $30^{\circ}$ and standard error larger than $10^{\circ}$. Coloured polygons represent features from Bell et al. (2010) and updated S2 from Barker et al. (2018); seamounts (S-red), high-amplitude reflectivity zones (H-blue) and low-amplitude reflectivity zone (L-green). Black contours show total slip during SSE2 (Wallace et al., 2016). Triangles are locations of offshore and onshore seismic stations. Red dashed line marks the Hikurangi Trench (Litchfield et al., 2014). Inset A shows sketch of the top view of a fracture network created by a subducting seamount, red circle, based on Dominguez et al. (1998). The red box highlights the area where fast azimuths resemble fracture patterns created by subducting seamounts. The black arrow shows the convergence rate between the Pacific and Central Hikurangi blocks [from Wallace et al. (2004)]. 


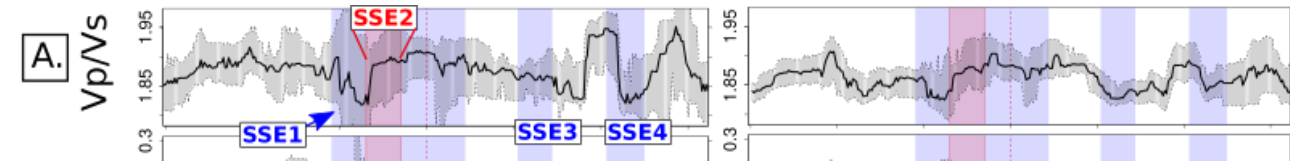

B. 5 。 SSE1 $\quad$ SSE3 $=$ SSE4

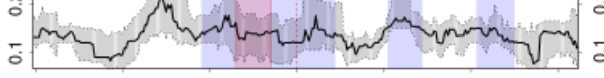

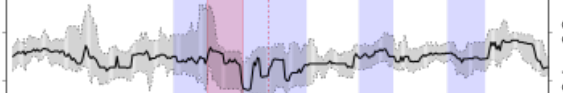

(250

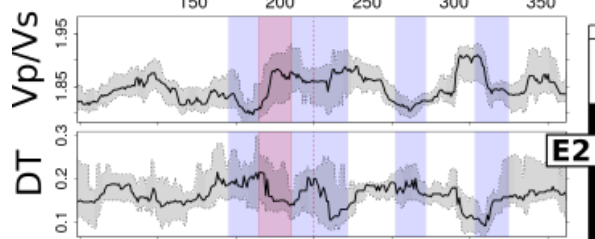

\&
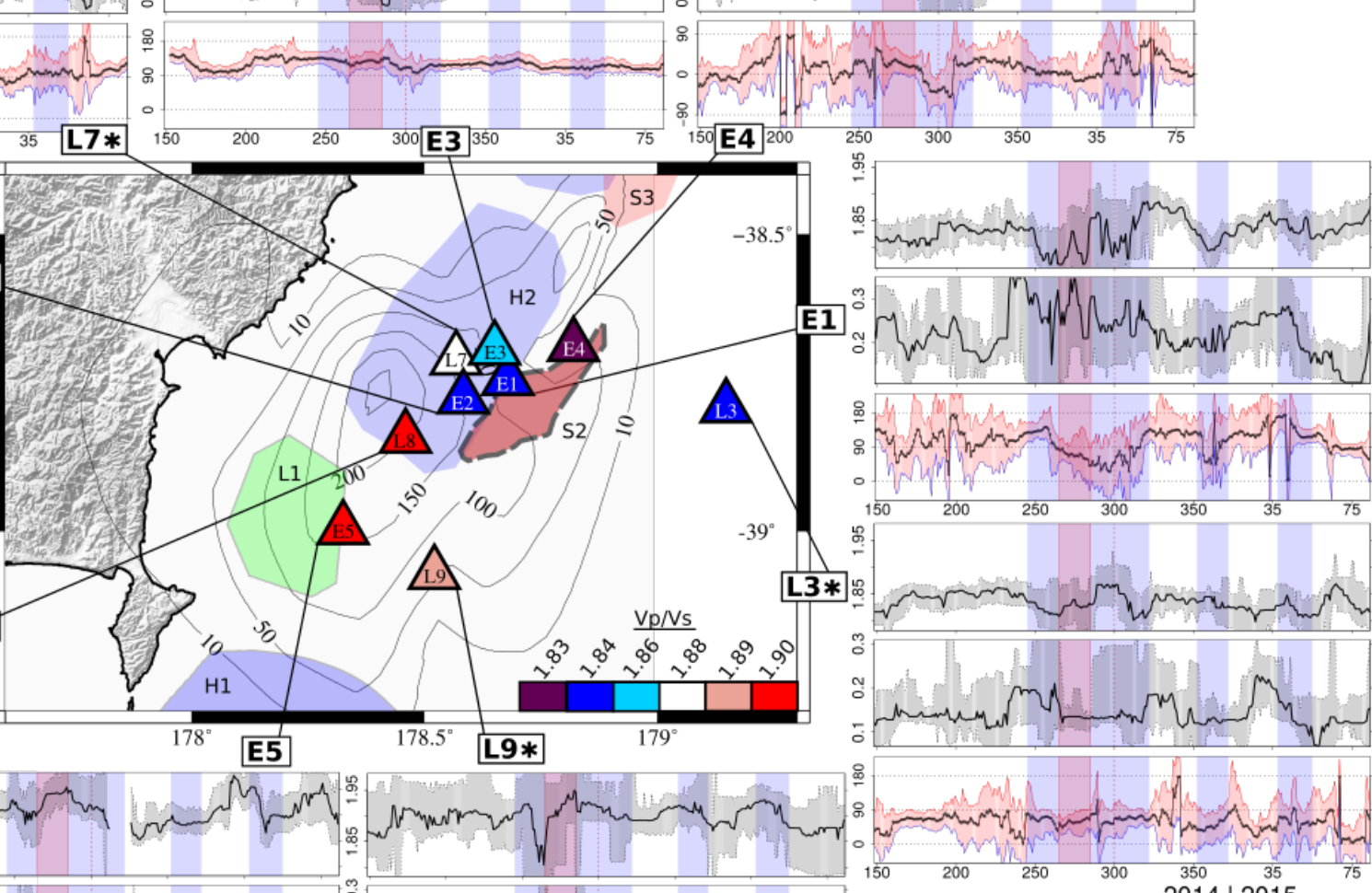

Julian Days

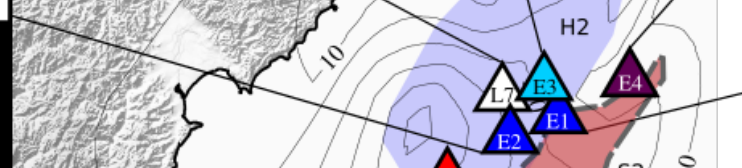

$-38.5$

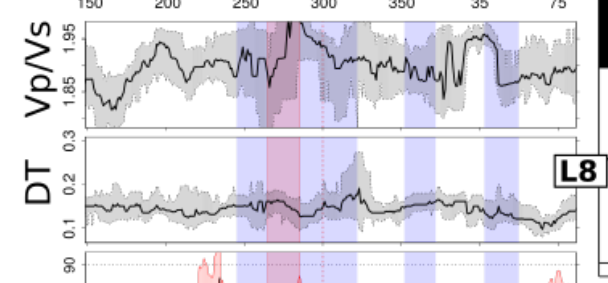

$$
\theta
$$

$150 \quad 200 \quad 250 \quad 300 \quad 350$

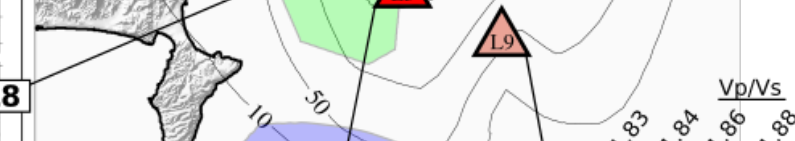

Julian Days

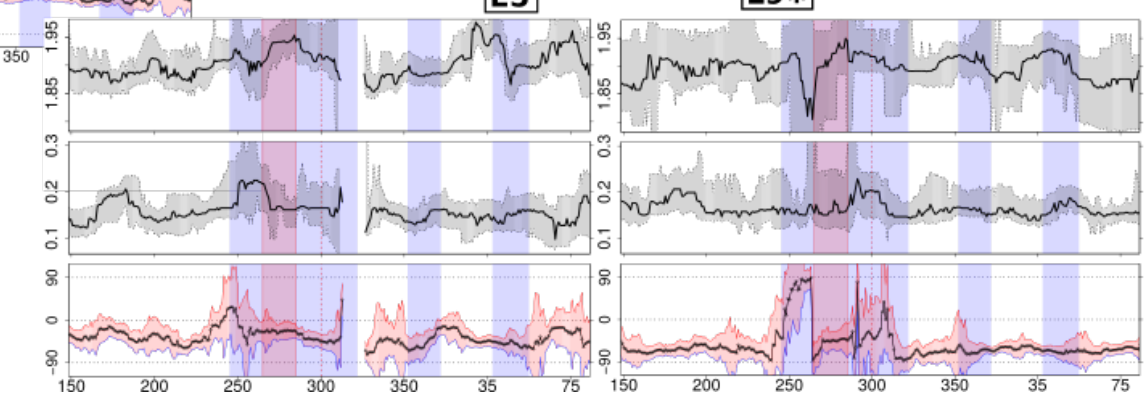

L3

L3*

\section{$\sqrt{8}$}

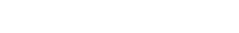

Figure 3.5: $V_{P} / V_{S}$ and SWS results at HOBITSS stations. On map- $S 2$ and $S 3$ are areas of seamounts, $H 1$ and $H 2$ are high-amplitude reflectivity zones and L1 is a lens reflectivity zone, mapped by Bell et al. (2010) and Barker et al. (2018). Triangles indicate LOBS and EOBS stations and are coloured by the average $V_{P} / V_{S}$. Stations marked with an asterisk had timing issues, although SWS results will not be impacted (refer to Appendix B, Figure B.1). (A) $V_{P} / V_{S}$. Black line is a median of 20-day moving windows with 95\% confidence intervals. The main pulse of the SSE2 is shaded in red and the red dashed vertical line marks the extended duration of the low-level slip associated with this SSE. Blue rectangles indicate times of other SSEs close to our study area. (B) Delay time. Black line is a median of 20-day moving windows with $95 \%$ confidence intervals. (C) Fast azimuths. Black line is a mean of 20-day moving windows with $95 \%$ confidence intervals. 
Table 3.1: Average $\Phi, V_{P} / V_{S}$, and delay time for individual stations

\begin{tabular}{|c|c|c|c|c|c|c|c|c|}
\hline Station & $\mathrm{N}$ & $\mu(\phi)$ & $95 \% \mathrm{CIs}$ & Rayleigh Test p-Value & Median $V_{P} / V_{S}$ & $95 \%$ CIs & Median $\delta t$ & $95 \%$ CIs \\
\hline LOBS3 & 490 & $59.7^{\circ}$ & {$\left[49.6^{\circ}, 69.1^{\circ}\right]$} & $1.839 \mathrm{e}-8$ & 1.835 & {$[1.829,1.843]$} & $0.135 \mathrm{~s}$ & {$[0.128 \mathrm{~s}, 0.150 \mathrm{~s}]$} \\
\hline LOBS7 & 1072 & $19.3^{\circ}$ & {$\left[12.8^{\circ}, 26.1^{\circ}\right]$} & $9.262 \mathrm{e}-17$ & 1.876 & {$[1.869,1.885]$} & $0.135 \mathrm{~s}$ & {$[0.124 \mathrm{~s}, 0.143 \mathrm{~s}]$} \\
\hline LOBS8 & 1083 & $-7.6^{\circ}$ & {$\left[169.0^{\circ}, 175.8^{\circ}\right]$} & $1.077 \mathrm{e}-51$ & 1.897 & {$[1.885,1.913]$} & $0.143 \mathrm{~s}$ & {$[0.139 \mathrm{~s}, 0.150 \mathrm{~s}]$} \\
\hline LOBS9 & 851 & $-64.6^{\circ}$ & {$\left[110.6^{\circ}, 120.1^{\circ}\right]$} & $1.340 \mathrm{e}-28$ & 1.891 & {$[1.884,1.906]$} & $0.161 \mathrm{~s}$ & {$[0.154 \mathrm{~s}, 0.170 \mathrm{~s}]$} \\
\hline EOBS1 & 695 & $-64.9^{\circ}$ & {$\left[99.1^{\circ}, 131.1^{\circ}\right]$} & $1.068 \mathrm{e}-3$ & 1.837 & {$[1.829,1.844]$} & $0.210 \mathrm{~s}$ & {$[0.195 \mathrm{~s}, 0.240 \mathrm{~s}]$} \\
\hline EOBS2 & 1144 & $-75.1^{\circ}$ & {$\left[-91.5^{\circ},-58.9^{\circ}\right]$} & $1.245 \mathrm{e}-3$ & 1.842 & {$[1.836,1.847]$} & $0.161 \mathrm{~s}$ & {$[0.154 \mathrm{~s}, 0.169 \mathrm{~s}]$} \\
\hline EOBS3 & 1146 & $-61.8^{\circ}$ & {$\left[114.0^{\circ}, 122.6^{\circ}\right]$} & $2.993 e-36$ & 1.861 & {$[1.855,1.868]$} & $0.148 \mathrm{~s}$ & {$[0.139 \mathrm{~s}, 0.154 \mathrm{~s}]$} \\
\hline EOBS4 & 889 & $8.2^{\circ}$ & {$\left[-3.2^{\circ}, 20.2^{\circ}\right]$} & $5.402 \mathrm{e}-6$ & 1.834 & {$[1.830,1.839]$} & $0.165 \mathrm{~s}$ & {$[0.154 \mathrm{~s}, 0.169 \mathrm{~s}]$} \\
\hline EOBS5 & 963 & $-36.7^{\circ}$ & {$\left[137.6^{\circ}, 149.2^{\circ}\right]$} & $1.305 \mathrm{e}-19$ & 1.899 & {$[1.891,1.907]$} & $0.158 \mathrm{~s}$ & {$[0.150 \mathrm{~s}, 0.165 \mathrm{~s}]$} \\
\hline CNGZ & 76 & $80.0^{\circ}$ & {$\left[17.8^{\circ}, 150.2^{\circ}\right]$} & 0.714 & 1.920 & {$[1.882,1.947]$} & $0.178 \mathrm{~s}$ & {$[0.150 \mathrm{~s}, 0.238 \mathrm{~s}]$} \\
\hline KNZ & 169 & $65.8^{\circ}$ & {$\left[59.1^{\circ}, 72.7^{\circ}\right]$} & $2.240 \mathrm{e}-13$ & 1.876 & {$[1.857,1.895]$} & $0.180 \mathrm{~s}$ & {$[0.155 \mathrm{~s}, 0.199 \mathrm{~s}]$} \\
\hline MHGZ & 61 & $69.9^{\circ}$ & {$\left[-161.5^{\circ},-11.1^{\circ}\right]$} & 0.664 & 1.873 & {$[1.850,1.900]$} & $0.240 \mathrm{~s}$ & {$[0.195 \mathrm{~s}, 0.270 \mathrm{~s}]$} \\
\hline MWZ & 385 & $54.6^{\circ}$ & {$\left[43.8^{\circ}, 66.5^{\circ}\right]$} & $9.374 \mathrm{e}-6$ & 1.810 & {$[1.803,1.818]$} & $0.124 \mathrm{~s}$ & {$[0.116 \mathrm{~s}, 0.135 \mathrm{~s}]$} \\
\hline PRGZ & 191 & $11.2^{\circ}$ & {$\left[3.6^{\circ}, 18.8^{\circ}\right]$} & $1.623 \mathrm{e}-10$ & 1.892 & {$[1.869,1.924]$} & $0.255 \mathrm{~s}$ & {$[0.219 \mathrm{~s}, 0.285 \mathrm{~s}]$} \\
\hline PUZ & 309 & $1.5^{\circ}$ & {$\left[-4.2^{\circ}, 7.1^{\circ}\right]$} & $1.664 \mathrm{e}-18$ & 1.817 & {$[1.799,1.836]$} & $0.176 \mathrm{~s}$ & {$[0.158 \mathrm{~s}, 0.200 \mathrm{~s}]$} \\
\hline RIGZ & 427 & $-6.3^{\circ}$ & {$\left[-15.2^{\circ}, 2.3^{\circ}\right]$} & $9.541 \mathrm{e}-9$ & 1.891 & {$[1.874,1.906]$} & $0.194 \mathrm{~s}$ & {$[0.176 \mathrm{~s}, 0.205 \mathrm{~s}]$} \\
\hline TKGZ & 437 & $83.0^{\circ}$ & {$\left[76.5^{\circ}, 90.0^{\circ}\right]$} & $1.439 \mathrm{e}-15$ & 1.875 & {$[1.855,1.891]$} & $0.146 \mathrm{~s}$ & {$[0.135 \mathrm{~s}, 0.154 \mathrm{~s}]$} \\
\hline TWGZ & 288 & $59.1^{\circ}$ & {$\left[46.6^{\circ}, 71.3^{\circ}\right]$} & $2.213 e-5$ & 1.851 & {$[1.829,1.870]$} & $0.180 \mathrm{~s}$ & {$[0.165 \mathrm{~s}, 0.195 \mathrm{~s}]$} \\
\hline
\end{tabular}

Note: N: Number of A and B grade measurements and $\mu(\phi)$ : circular mean of the fast azimuth (relative to North). $95 \%$ CIs: $95 \%$ confidence intervals given as [lower limit, upper limit]. 
Because the majority of raypaths travel through or directly above the SSE patch, we stack the temporal $V_{P} / V_{S}$ and SWS delay time measurements to help reinforce variations seen at individual stations (Figure 3.6). The stacking highlights a regional decrease in $V_{P} / V_{S}$ starting at Julian day 248, from 1.860; CIs [1.844, 1.872] to 1.817; CIs [1.806, 1.830], over the 15 days leading up to SSE2. During SSE2 $V_{P} / V_{S}$ increases from 1.817 to 1.894 ; CIs [1.882,1.907]. Following the main slip pulse (Julian day 285), $V_{P} / V_{S}$ decreases back to 1.833; CIs [1.826, 1.842] around Julian day 11, 2015.

The delay times show an inverse trend to $V_{P} / V_{S}$ and less variation overall (Figure 3.6). Prior to SSE2, the delay time increases from 0.143s; CIs [0.131s, 0.154s] to 0.178s; CIs [0.206s, 0.158s], over 50 days. During the main SSE slip pulse, the delay time decreases from 0.178 s to $0.139 \mathrm{~s}$; CIs [0.128s, $0.150 \mathrm{~s}]$. The delay time then returns to the initial value of $\sim 0.156$ over $\sim 50$ days. Similarities in the moving medians of $V_{P} / V_{S}$ and delay time observations suggest that variations associated with the occurrence of SSEs are a regional process that can be widely observed.

To test the effects on $V_{P} / V_{S}$ and delay time by stacking stations located on the outskirts of SSE2, we compare our results from stacking all the stations to only the stations (LOBS7, LOBS8, EOBS2, EOBS5) located in the center of the SSE and stations (LOBS3, LOBS9, EOBS1, EOBS3, EOBS4) located outside of the SSE (Appendix B, Figure B.3). We observe a similar trend in the different station distributions, although the trend for the stack of stations located closest to the center of the SSE shows a stronger signal in both $V_{P} / V_{S}$ and delay time, suggesting that the area of highest slip has the greatest effect on our observed measurements.

The second largest change in $V_{P} / V_{S}$ is observed starting on day 26 (2015; Figure 3.6), with values increasing from 1.833; CIs [1.826, 1.842] to 1.892; CIs [1.879, 1.906] and has no matching signal in the delay time measurements. To investigate the isolated $V_{P} / V_{S}$ change we examined earthquakes during this time and found a cluster of 31 onshore earthquakes between Julian days 36-39, 2015 (Appendix B, Figure B.4). Earthquakes located in the same area as this cluster but at different times produce high $V_{P} / V_{S}$ values, close to 1.9. The 20-day length of the moving window, plotted at the center of the window, causes a 10-day shift in the start of the associated $V_{P} / V_{S}$ increase. To test for the influence of the cluster on $V_{P} / V_{S}$, we removed it and the resulting $V_{P} / V_{S}$ no longer shows an increase (Appendix B, 


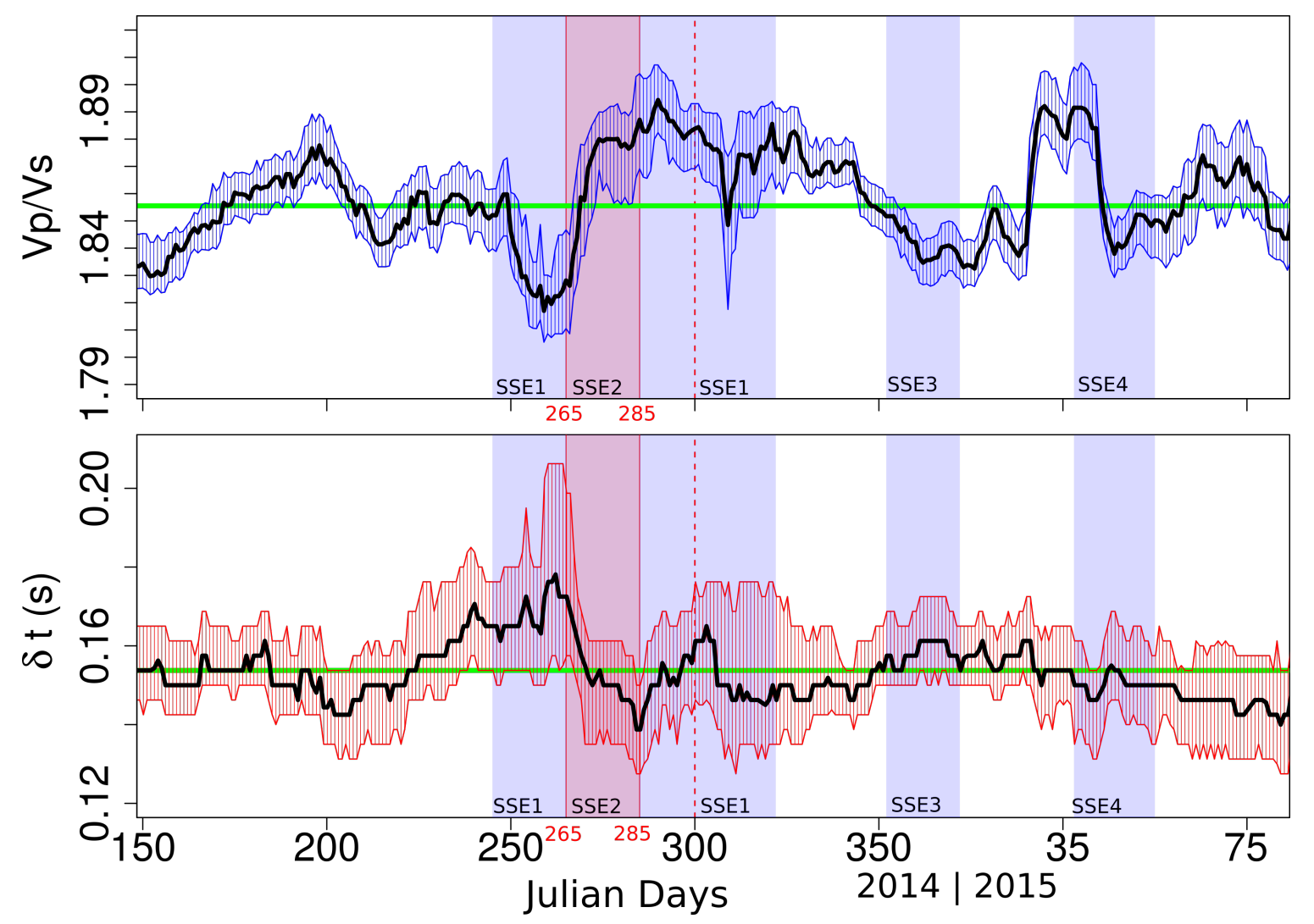

Figure 3.6: Top: $V_{P} / V_{S}$ change on stack of all offshore stations for entire catalog (9,056 measurements). Blue lines indicate upper and lower 95\% confidence intervals. Horizontal green line shows median $V_{P} / V_{S}$ for all measurements. SSEs are shaded and labeled 1-4. The main pulse of SSE2 is shaded red. The red dashed vertical line marks the extended duration of the low-level slip associated with SSE2. Bottom: Delay time change on stacked offshore stations (8,333 measurements). Red lines indicate upper and lower 95\% confidence intervals. Green line shows median delay time for all measurements.

Figure B.4). The lack of change of delay time with $V_{P} / V_{S}$ indicates that the inverse variation in the delay time signal is essential to distinguishing physical changes from spurious variations in the data.

To test for temporal variations due to variations in earthquake locations we examine sub-catalogs of 159 earthquakes that have near-identical locations at different times (Appendix B, Section B.3, Figure B.5). Figure B.6 shows the stack of earthquakes using our spatial sub-catalogs from Figure B.5 for offshore (E-F) and onshore $(\mathrm{G}-\mathrm{H})$ stations. We observe similar temporal variations in $V_{P} / V_{S}$ and $\delta t$ from earthquakes occurring in our spatial sub-catalogs compared to using earthquakes from the entire catalog for offshore stations, although the confidence intervals of the former are larger (Figure B.6). This result suggests that spatial variations in 
earthquake locations are not a major contributor to the observed $V_{P} / V_{S}$ and $\delta t$ temporal variations.

In laboratory studies, apparent $V_{P} / V_{S}$ ratios vary considerably by changing the raypath angle, between perpendicular and parallel, relative to the crack fabric (Wang et al., 2012). However, we do not observe significant changes in $V_{P} / V_{S}$ related to varying incidence angle (Appendix B, Figure B.7) and thus do not consider incidence angle as a contributor to the observed temporal changes.

\subsection{Discussion}

Most stations have a significant mean fast azimuth (Figure 3.3; Table 3.1). Mean fast azimuths from previous results by Unglert (2011), at onshore stations PUZ, KNZ, and MWZ (Figure 3.1), closely match the mean fast azimuths obtained in this study for the same stations (Figure 3.3). Onshore stations tend to exhibit NS or NE-SW azimuths and are oriented parallel or sub-parallel to nearby normal faults (Figure 3.3; Litchfield et al., 2014).

Spatial averaging (Figure 3.4) helps to reveal trench perpendicular fast azimuths, NW-SE, in the southeastern portion of the array, east of Mahia peninsula, into the slip region, and across stations EOBS5 $\left(323^{\circ}\right.$; CIs $\left.\left[317^{\circ}, 329^{\circ}\right]\right)$ and LOBS9 $\left(296^{\circ}\right.$; CIs $\left[291^{\circ}, 301^{\circ}\right]$ ) and the lens reflectivity zone (Bell et al., 2010), L1 (Figure 3.3). The NW-SE fast azimuths are sub-parallel to the convergence direction near the trench, $\sim 294^{\circ}$ (Pacific and Central Hikurangi blocks; Wallace et al., 2004), suggesting that regional stresses, induced by plate convergence, are dominant in this region.

The fast azimuths around the seamount $S 2$ resemble fracture and fault patterns previously identified around subducting seamounts in both field and laboratory studies (Figure 3.4; Inset A; Dominguez et al., 1998). Seamount S2 spans an area of $\sim 30 \mathrm{~km}$, and the observed variable fast azimuths extend around the downdip edge of the seamount, where stresses are expected to be highest (Baba et al., 2001). The location of the high-amplitude reflectivity zone, $H 2$, is consistent with shear zone deformation in front of subducting seamounts (Baba et al., 2001; Bell et al., 2010). The resemblance of the fast azimuths to seamount fracture patterns indicates that these directions may be structurally controlled through faulting. The complex fracture pattern and stress distribution created by subducted seamounts creates numerous connected fluid pathways that could promote slow slip around 
the seamount (Shaddox and Schwartz, 2019). Todd et al. (2018) found that tremor during SSE2 is localized in the vicinity of seamount S2 and S3 and suggest that tremor may be triggered by stress changes induced by slow slip (Barker et al., 2018). There are several smaller subducted seamounts off the coast of Gisborne located in our study area (Bell et al., 2010), although the raypath coverage across these seamounts is limited. Seamount S2 is the largest in the SSE rupture area and the HOBITSS array.

Although most stations have significant mean fast azimuths, temporal variations in these azimuths are observed on stations EOBS1, 4 and LOBS9 (Figure 3.5). Stations EOBS1 and EOBS4 exhibit a gradual anti-clockwise rotation in $\Phi$ during SSE2, starting at $\sim 160^{\circ}$ at Julian day 260 , decreasing to $\sim 40^{\circ}$ by day 290 and completing a full $180^{\circ}$ circuit by returning to $\sim 160^{\circ}$ on day 300 (Figure 3.5). Stations EOBS1 and 4 are in close proximity to the intersection point between seamount $S 2$ and high-amplitude reflectivity zone $\mathrm{H} 2$ and we suggest that their temporal changes are related to SSE2 and the position of these sites relative to the seamount.

The southernmost offshore station, LOBS9, is located on a bend within the slip contours and shows a clear change in $\Phi$ starting at Julian day 245, 20 days before SSE2 (Figure 3.5). Calculations for the Coulomb failure stress change on the plate interface show a large change in stress on the bend in the slip contours near station LOBS9 (Todd et al., 2018). This change in the fast azimuth at LOBS9 might be a precursory signal, however, the change also coincides with the onset of SSE1, just south of our study area and before SSE2 (Warren-Smith et al., 2019). We cannot distinguish between a precursory signal or a stress change associated with SSE1, to the south. Additionally, the 20-day moving windows used to plot the $V_{P} / V_{S}$, delay time, and $\Phi$ are plotted at the center of the window, so any observed changes could have started 10 days earlier or later. Although the 20-day moving windows obscure the timing of changes by \pm 10 days, inspection of 1-day moving windows indicates that the change in parameters does begin on day 245, 20 days before SSE2.

Crustal stress changes can occur on both a local scale, induced by earthquakes, and a regional scale, caused by tectonic loading. If local and regional stresses are on the same order as, or stronger than that of structural influences, and $S_{H} \max$ is not parallel to the strike of the structure, it is possible to observe changes in $S_{H} \max$ by 
monitoring temporal changes in $\Phi$ as it is sensitive to the shape, orientation, and density of microcracks. Changing fast azimuths on stations EOBS1, 4 and LOBS9 are interpreted as localized rotations in microcracks due to the stress changes associated with SSEs. The lack of changes in $\Phi$ at stations that are in close proximity to stations that do exhibit a rotation suggest that stress changes in the crust may be highly localized and channelled by existing fault structures. Local and regional changes in stress or fluid content can also cause fluctuations in delay times and $V_{P} / V_{S}$.

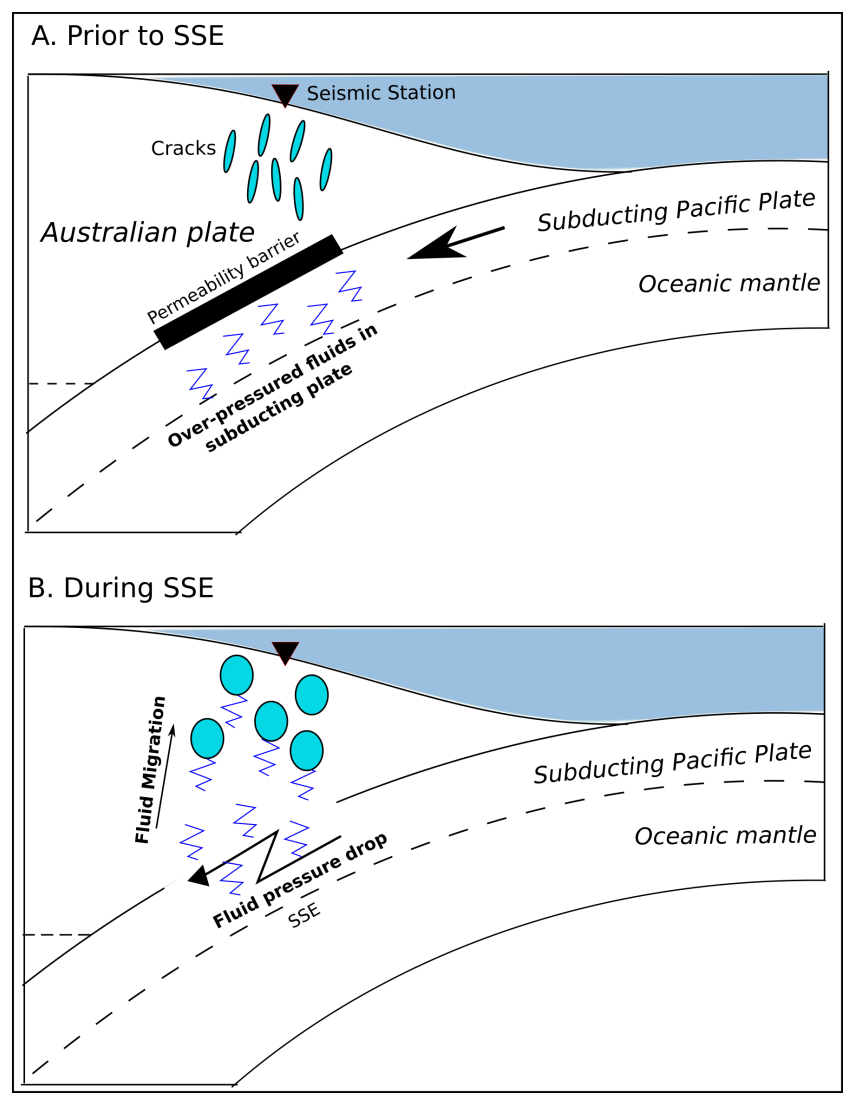

Figure 3.7: Model adapted after Husen and Kissling (2001) explaining fluid flow after the September-October 2014 SSE. Black arrow shows direction of stress along the seismogenic zone. A. Before the SSE rupture, increased stress due to convergence between plates. Near-lithostatic pore pressure pressures exist below the permeability boundary. B. After the SSE, permeability seal ruptures and fluids migrate upwards.

Our $V_{P} / V_{S}$ value of 1.73 for the catalog, derived from the slope of the Wadati plot (i.e., $T_{S}-T_{P}$ vs $T_{P}$ for all the combined data), is similar to the $V_{P} / V_{S}$ of 1.76 reported by Yarce et al. (2019) and the values from the 3D velocity of Eberhart-Phillips et al. (2010) which range from 1.75 to 1.80 in our study area. However, our Wadati plot has a $T_{P}$ intercept of $\sim 1$ second (at $T_{P}=0, T_{S} / T_{P}=1.0 \mathrm{~s}$ ). The $\sim 1$ second offset in- 
dicates that earthquakes travel through material with different values of Poisson's ratio (Kisslinger and Engdahl, 1973). This is likely caused by slow shear velocity sediments beneath the seafloor as recently modelled by Kaneko et al. (2019) to explain observations of ultra-long duration ground motion observed offshore Gisborne during the 2016 Kaikoura earthquake. The material properties and intercept offset impact event-station $V_{P} / V_{S}$ measurements as the calculation of individual $T_{S} / T_{P}$ values assumes an intercept at the origin. We therefore obtain increased $V_{P} / V_{S}$ from individual measurements, with the median (1.857) larger than the overall catalog $V_{P} / V_{S}$ (1.73), from the slope of the Wadati plot.

Most offshore stations exhibit a decreasing delay time and increasing $V_{P} / V_{S}$ during SSE2 (Figure 3.5). Because the majority of raypaths travel through the SSE patch, we stack the temporal delay times and $V_{P} / V_{S}$ measurements to help reinforce variations seen at individual stations (Figure 3.6). Prior to SSE2, the delay time increases from 0.143 s to 0.178 s, over $\sim 50$ days, while the $V_{P} / V_{S}$ decreases from 1.860 to 1.817 , over $\sim 15$ days leading up to SSE2. The delay time and $V_{P} / V_{S}$ change before SSE2 suggests there may be a recognizable precursory signal to SSEs. However, SSE1, occurring just south of our study area coincides with the change in $V_{P} / V_{S}$ and delay time and could potentially influence these measurements. We interpret this trend in $\delta t$ and $V_{P} / V_{S}$ as changes in differential horizontal stress that act to change crack aspect ratios and orientations. Differential horizontal stress will preferentially close the cracks that are oriented orthogonal to the maximum horizontal compressive stress $\left(S_{H} \max\right.$; Nur, 1971). With increasing differential horizontal stresses, cracks are compressed and become vertically aligned (Crampin, 1981). A ray traveling through vertically aligned cracks will result in maximum anisotropy and increased delay times (Nur, 1971). This is consistent with our observation of increasing $\delta t$ and decreasing $V_{P} / V_{S}$ values before the initiation of SSE2. Gao and Crampin (2006) previously observed increasing delay times before large earthquakes and also suggest stress accumulation as a source of the increase. However, they report that following that increase, some stations exhibit a decrease in delay time, before the earthquakes, suggesting crack coalescence as an interpretation of this phenomenon. We do not observe a decreasing delay time before SSE2 and suggest stress accumulation as the main contributor to our increasing delay times before the SSE. Crack coalescence may explain the decreasing delay time during SSE2. 
During SSE2, we observe a decrease in delay time, from 0.178 s to 0.139 s (Figure 3.6). The delay time then returns to a value of $\sim 0.156$ s over $\sim 50$ days. While delay time decreases during SSE2, $V_{P} / V_{S}$ increases, from 1.817 to 1.894 , followed by a decrease after the SSE back to 1.833, around Julian day 11, 2015. Previous studies showing similar $V_{P} / V_{S}$ changes have suggested that the rupture of a lowpermeability seal along the plate interface driven by large earthquakes (Husen and Kissling, 2001) or SSEs (Nakajima and Uchida, 2018) could explain the variations. Lucente et al. (2010) reported changes in $V_{P} / V_{S}$ linked to fluid flow across a faulted barrier, and also observed an inverse variation between $V_{P} / V_{S}$ and delay times. A thermomechanical fluid model along 05CM-04 line in Figure 3.1 predicts that significant over-pressures may develop in the subduction interface if a lowpermeability $\left(10^{-20} \mathrm{~m}^{2}\right)$ seal is present (Ellis et al., 2015). Recent work by WarrenSmith et al. (2019) using the HOBITSS dataset suggests that observed fluctuations in retrieved stress ratios inferred from earthquakes may indicate changes in pore fluid pressure within the subducting crust (below a possible low-permeability seal) that is related to, or perhaps controls, SSE timing. Shaddox and Schwartz (2019) observe repeating earthquakes occurring during SSE2, on multiple upperplate faults within the fracture network of subducted seamount $S 2$. They suggest that these earthquakes were triggered by the migration of fluid during the SSE from over-pressured sediments downdip of the seamount into the upper-plate.

Similar to Warren-Smith et al. (2019), we suggest that when SSE2 initiated, a low permeability seal was ruptured, allowing for fluid migration and the interconnection of fluids (i.e., crack coalescence) that were previously isolated (Figure 3.7). After the rupture of the permeability barrier, the pressure gradient between possible near-lithostatic pore pressure in the subducting plate/plate interface and relatively lower pore pressure in the overriding plate induces fluid transfer. The increase in $V_{P} / V_{S}$ and decrease in delay time during SSE2 suggests that an interconnection of cracks allows for fluid transfer upwards from below the previously sealed, possibly over-pressured subducting plate. This is consistent with previous analysis of cyclic stress tensors and inferred fluid pressure changes observed from focal mechanisms analysis (Warren-Smith et al., 2019), which suggests a drop in fluid pressure occurring within the subducting crust during multiple SSEs recorded during HOBITSS. With horizontal differential stresses decreasing after the onset of SSE2 and with fluids migrating upwards, cracks become rounder and more randomly 
oriented, consistent with a decreasing delay time and increasing $V_{P} / V_{S}$. After SSE2, we interpret that the drop in $V_{P} / V_{S}$ is due to drainage of fluids from the previously fluid-rich zone.

A similar trend to our delay time and $V_{P} / V_{S}$ was observed during the 2009 L'Aquila earthquake sequence in Italy. Lucente et al. (2010) observed a sudden change in $V_{P} / V_{S}$ on several stations after the largest-magnitude foreshock. They infer that the seal between the footwall and hanging wall was broken after the foreshock, promoting fluid migration across the fault zone and filling the fractures and cracks in the hanging wall. A station located above the hanging wall of the fault exhibits a $V_{P} / V_{S}$ rising from $\sim 1.8$ to 1.85 and decreasing back to 1.83 and a normalized delay time increasing $~ 15$ days before the ML 4 foreshock and sharply decreasing after the foreshock.

As discussed in the introduction, Husen and Kissling (2001) interpret the increase in $V_{P} / V_{S}$ ratios (over 50 days) following the main shock of the Mw 8.0 earthquake in Antofagasta, Chile as evidence of post-seismic fluid flow after the rupture of a permeability seal during the main shock of the earthquake, with near-lithostatic fluid pressures below the sealed boundary (prior to the seal breaching earthquake) causing fluids to migrate above the rupture zone, into the upper-plate. Nakajima and Uchida (2018) observe variations in seismic attenuation and seismicity rates with the cyclic occurrence of SSEs in Kanto, Japan. They proposed that a lowpermeability seal surrounding the megathrust fails during SSEs and drainage saturates the rock above, resulting in increased supraslab seismicity and attenuation in the area (Nakajima and Uchida, 2018). The break is then resealed by cementation and pore-fluid pressures in the megathrust begin to increase, eventually leading to the next phase of failure. Our $V_{P} / V_{S}$ and delay time changes support suggestions by Warren-Smith et al. (2019) that a similar process may be at work in Hikurangi.

Husen and Kissling (2001) report an estimated permeability value of $10^{-16}-10^{-17} \mathrm{~m}^{2}$, given a time period of 50 days between the main shock and the appearance of the high $V_{P} / V_{S}$ ratios and a fluid migration distance of $20 \mathrm{~km}$. Nakajima and Uchida (2018) estimate a permeability of $2-4 \times 10^{-14} \mathrm{~m}^{2}$ for a time range of $0.2-0.4$ years and a fluid migration distance of $5 \mathrm{~km}, 2-3$ orders of magnitude larger compared to that estimated by Husen and Kissling (2001). To estimate the permeability in the 
September-October 2014 SSE (SSE2) region, we use the relation from Townend and Zoback (2000) and modified by Nakajima and Uchida (2018), $k=\beta \eta l^{2} / \tau$. Permeability $(k)$ is expressed as a function of time $(\tau)$, fluid migration distance $(l)$, specific storage coefficient $(\beta)$, and dynamic viscosity $(\eta)$. Given a time of 20 days, in which the $V_{P} / V_{S}$ increased during SSE2, a fluid migration distance between 0.5-4 $\mathrm{km}$, based on the range of depths where the majority of the repeating earthquakes occurred (e.g., 3-7 km depth; Shaddox and Schwartz, 2019), ( $\beta$ ) of 10-10 $\mathrm{Pa}^{-1}$ for low porosity rock, and dynamic viscosity $(\eta)$ of $10^{-4} \mathrm{~Pa} \mathrm{~s}$, we obtain a permeability between $0.1 \times 10^{-14}$ and $9 \times 10^{-14} \mathrm{~m}^{2}$. Such high permeability values suggest a highly fractured network of cracks in the overriding plate. Despite the slow rupture velocity, we propose that the September-October 2014 SSE was capable of rupturing enough of the permeability seal to induce fluid flow, consistent with our observed increase in $V_{P} / V_{S}$.

\subsection{Conclusions}

We calculated SWS and $V_{P} / V_{S}$ ratios for event-station pairs using local earthquakes recorded, for the first time, on OBSs located above a shallow, 5-week long, offshore SSE on the Hikurangi subduction zone. Our findings suggest that spatial variations in fast azimuths are affected by stress and structures in the region and that stress changes and fluid migration during SSEs are detectable with SWS and $V_{P} / V_{S}$ analyses.

Spatial averaging shows regional stress directions in much of the offshore area, parallel to the relative plate convergence directions, but with a variation around subducted seamount, 52 . This variation is consistent with fault and fracture patterns created by subducting seamounts observed in laboratory and field experiments. The resemblance of the fast azimuths to seamount fracture patterns suggests some structural control through faulting. The upper-plate fracture network above the subducted seamount is the host of migrating fluids, from over-pressured sediments downdip of the seamount, resulting in a complicated environment of stress distribution (Shaddox and Schwartz, 2019).

Temporal changes in fast azimuths at individual stations are observed around the time of the main SSE. Of these stations, EOBS1 and 4, located on the border between seamount, $S 2$, and a high-amplitude reflectivity zone, $H 2$, show a 
change occurring during the September-October 2014 SSE (SSE2). We interpret the changes in $\Phi$ at these stations as a rotation in microcracks corresponding to stress changes associated with SSEs. The temporal variations in $\Phi$ at individual stations, along with the similarity of spatial averaging fast azimuths to structural patterns around the subducting seamount, suggest a combination of stress and structural effects due to the interactions of subducting seamounts with the over-riding plate. The lack of observed changes at other nearby stations indicate that areas of stress change can be highly localized and complex.

Temporal variations in $V_{P} / V_{S}$ and delay time measurements are observed on individual OBS stations during the September-October 2014 SSE. Stacking measurements across all offshore stations enhanced our signal and revealed clear opposing trends in delay time and $V_{P} / V_{S}$. The stacked signal also indicates that the changes may occur over a broad region. Similar to Warren-Smith et al. (2019), we suggest that during the SSE a low permeability seal on the plate interface is ruptured, allowing for the interconnection of fluids. Shaddox and Schwartz (2019) propose that repeating earthquakes observed above seamount $S 2$ were triggered by fluid migration from over-pressured sediments downdip of the seamount into the upper-plate during the SSE. The increase in $V_{P} / V_{S}$ and decrease in delay time during the SSE suggests fluid transfer from the subducting plate, below the sealed fault zone. These observations are consistent with fluctuations in retrieved stress ratios, which indicate changes in pore fluid pressure in the subducting crust, during multiple SSEs recorded by the HOBITSS experiment (Warren-Smith et al., 2019). Following the end of SSE2, a decreasing $V_{P} / V_{S}$ may indicate the drainage of fluids from the over-pressurized fluid-rich crust. Our observations support the suggestion that the September-October 2014 SSE may have ruptured a permeability seal, and induced fluid flow.

Our findings show that temporal changes in $V_{P} / V_{S}$, delay time, and fast azimuths are detectable during SSEs and provide further evidence that fluids and stresses play a significant role in SSE nucleation and propagation. The inverse variation of $V_{P} / V_{S}$ and delay time measurements allows distinction between physical variations and variations in data. We recommend that future SSE monitoring studies should consider similar network configurations to study events of similar size. Larger regional networks could help define the spatial extent of observed changes. These results also demonstrate that SWS and $V_{P} / V_{S}$ are effective tools for investi- 
gating stress changes associated with slow slip.

\subsection{Acknowledgments}

We acknowledge support from an MBIE Endeavour Fund grant (funding contract C05X1605). Additional funding support came from National Science Foundation (NSF) grants: NSF-1551683, OCE-1333025, OCE-1334654, and OCE-1551922. K.M. was funded by ERI JURP 2013-B-09. The ocean bottom seismic data were provided by instruments from the Ocean Bottom Seismograph Instrument Pool (www.obsip.org) funded by the NSF, the Earthquake Research Institute (ERI), University of Tokyo, and New Zealand's GeoNet project. Raw data from the experiment are archived at Incorporate Research Institutions for Seismology Data Management Center (IRIS-DMC) with experiment codes YH 2014-15 (seismic data) and 8F 2014-15 (bottom pressure record data).

We would like to acknowledge the earthquake database used in this study and put together by Jefferson Yarce. The database is publicly available and can be found at https://zenodo.org/record/2022405\#.XIbszRNKgWo. A table of our shear wave splitting and $V_{P} / V_{S}$ results can be found at https://zenodo.org/record/3383417\#.XWyko3Uzbys. We thank ERI for providing Hubert Zal and Martha Savage with fellowships as visiting researchers and stimulating valuable discussion that significantly improved the manuscript. We would also like to thank Emily-Warren Smith and Carolyn Boulton for their helpful feedback and discussion. 


\section{Chapter 4}

\section{Spatial variations in seismic anisotropy in central North Island,}

New Zealand

\subsection{Abstract}

We measured shear wave splitting (SWS) parameters using 20,835 local earthquakes recorded by 24 stations of the GeoNet Network (2009-2012). The resulting 42,423 high-quality SWS measurements provided us with one of the largest datasets of crustal anisotropy measurements in central North Island, New Zealand. Our goal was to improve our understanding of the relationship between the stress state and crustal seismic anisotropy across central North Island. To accomplish this, we used SWS to examine spatial variations in fast polarization azimuths and delay times. We compared our SWS fast polarization azimuths to stress orientations derived from continuous campaign GPS and gravitational stress calculations, as well as orientations of active faults. The spatial averaging of SWS fast polarization azimuths showed significant spatial variations across central North Island. Comparisons with other measurements helped reveal contributing sources of influence. The fast azimuths at many stations across the North Island Dextral Fault Belt (NIDFB) were consistent with the regional NE-SW fault orientations suggesting an overall strong structural control on anisotropy.

However, we found significant deviations from this structural trend in two re- 
gions, namely in the Wanganui Basin and a small area along the NIDFB. There, the fast azimuths tended to correlate with the regional maximum compressional stress $S_{H} \max$. Additionally, the fast azimuths around Mt. Ruapehu showed complex variations and resemblance to both $S_{H} \max$ and fault orientations, suggesting a combination of both stress-induced and structurally-controlled anisotropy in the Mt. Ruapehu region. Delay time spatial averaging revealed an increase in delay times east of the NIDFB, which was consistent with increased $V_{P} / V_{S}$ values closer to the subduction interface, suggesting increasing horizontal stresses and/or crack densities towards the Hikurangi trench.

\subsection{Introduction}

The Hikurangi subduction zone in North Island, New Zealand, provides a useful laboratory to study the stress field in the crust. Several studies have systematically analyzed the stress field across North Island (e.g., Townend et al., 2012; Lamb, 2015; Hirschberg et al., 2019) and it has long been thought that the stress field may be an important factor in seismic anisotropy (e.g., Nur and Simmons, 1969; Zinke and Zoback, 2000; Boness and Zoback, 2006; Johnson et al., 2011).

In this chapter, we investigate the spatial relationship between seismic anisotropy and the stress state across central North Island from shear wave splitting (SWS) measurements for 20,835 local earthquakes from June 2009 to October 2012. Using local earthquake data recorded on 24 of GeoNet's permanent seismic stations, we created one of the largest catalogues of crustal anisotropy measurements across central North Island (Figure 4.1). Using these data, we compared our SWS measurements with both stress models and to other measurements to elucidate the potential contributing sources of influence controlling our measured anisotropy. The initial aim of this study was to search for temporal changes in the state of the crust during the 18-month long 2010-2011 Manawatu slow slip event (SSE). However, we did not find any significant temporal variations in our SWS results.

\subsubsection{Tectonic setting}

In North Island, New Zealand, the Pacific plate subducts westward beneath the Australian plate at $\sim 40-45 \mathrm{~mm} / \mathrm{yr}$, oblique to the plate boundary (DeMets et al., 1994; Beavan et al., 2002; Altamimi et al., 2012). The tectonics of the central North 


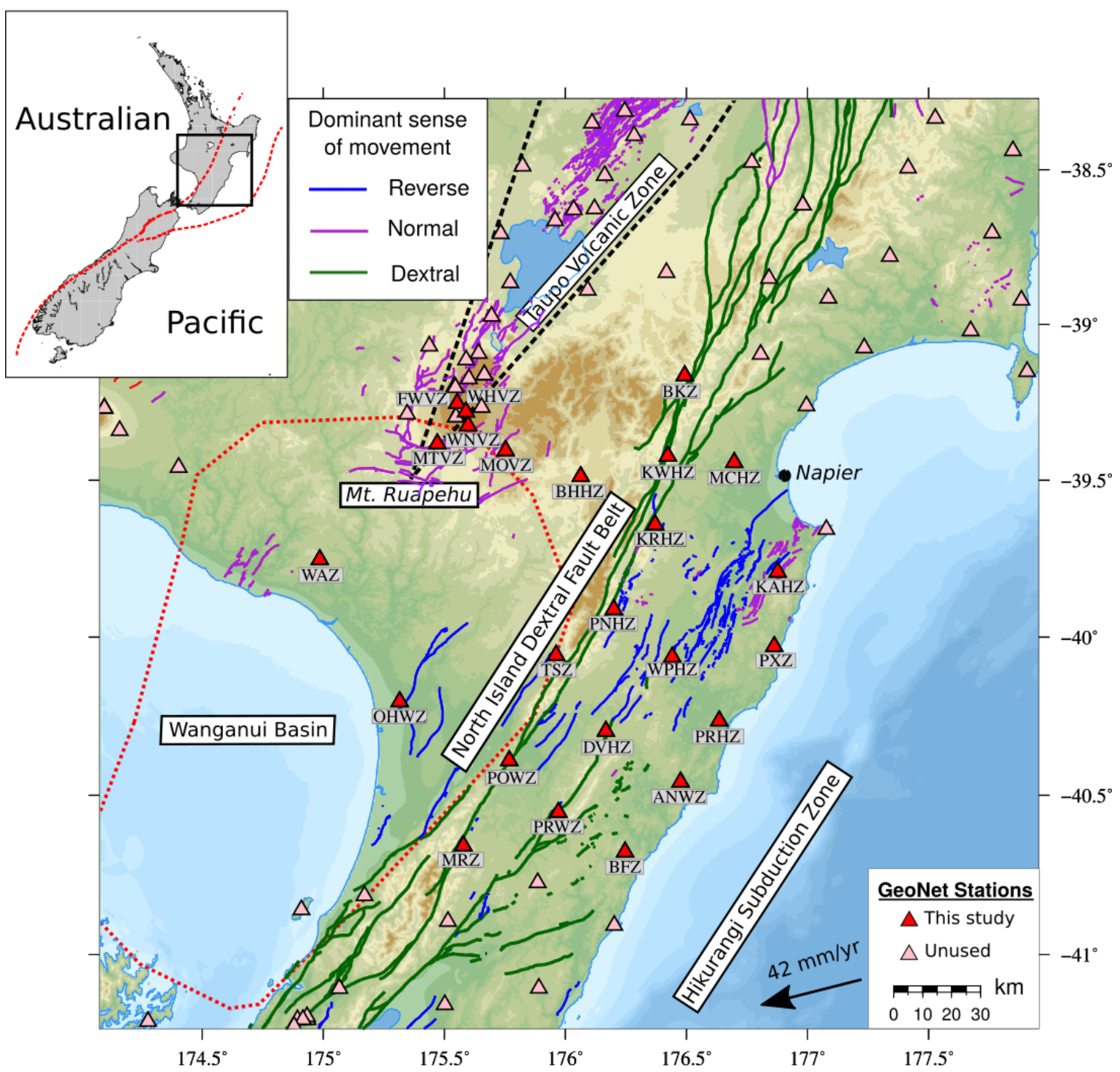

Figure 4.1: Locations of GeoNet seismic stations in central North Island, New Zealand. Red triangles mark locations of stations used in this study. Pink triangles mark unused stations. Active fault traces are from Langridge et al. (2016). The extent of Wanganui Basin is marked by a dotted red line (Tozer et al., 2017). The Taupo Volcanic Zone is marked by a dashed black line (Wilson et al., 1995). The black arrow shows the velocity of the Pacific plate relative to the Australian plate (Altamimi et al., 2012).

Island are dominated by subduction along the Hikurangi Margin, back-arc rifting in the Taupo Volcanic Zone (TVZ), strike-slip faulting in the North Island Dextral Fault Belt (NIDFB), and subsidence in the Wanganui Basin (Figure 4.1; Walcott, 1984; Beanland and Haines, 1998; Stratford and Stern, 2006). The TVZ forms the southern end of the Havre Trough, where backarc extension in the last 5 Ma has occurred between the Kermadec-Tonga subduction zone (Walcott, 1984). Mt. Ruapehu, at the southern end of the TVZ, marks the transition point where the hor- 
izontal stress regime changes from extensional to predominantly compressional to the south (e.g., Rowland and Sibson, 2001; Lamb, 2015; Hirschberg et al., 2019). The NIDFB takes up a significant portion of margin parallel plate motion by strikeslip faulting south of the TVZ, subparallel to the Hikurangi trench (Beanland and Haines, 1998). The backarc sedimentary basin, known as the Wanganui Basin, lies behind the southern part of the Hikurangi subduction zone, consisting of a thick sequence of sediments created by rapid subsidence (up to $5 \mathrm{~km}$ thick; Harmsen, 1985). Several mechanisms have been suggested for the crustal down-warp causing this subsidence, including plate interface frictional stresses (Stern et al., 1992), lithospheric thickening (Stern et al., 2006), and mantle flow forces (Kudo and Yamaoka, 2003).

\subsubsection{Slow slip events}

The Manawatu region is known to host the largest, longest and deepest SSEs in New Zealand. In 2004/2005 an SSE initiated at a depth of 35-60 km and propagated updip to 25-32 km depth over 18 months (Wallace and Beavan, 2006). This SSE migrated over a distance of $\sim 40 \mathrm{~km}$ along the strike of the margin. Seven continuous Global Positioning System (cGPS) sites recorded deformation of up to $36 \mathrm{~mm}$ of horizontal and $28 \mathrm{~mm}$ of vertical displacement (Wallace and Beavan, 2006). The 2010 Manawatu SSE evolved over 16 months and ruptured much of the plate interface between 20 and $70 \mathrm{~km}$ depth (Wallace et al., 2012b). Compared to the 2004/2005 SSE, the 2010/2011 SSE has been better constrained due to the use of a significantly larger array of cGPS sites during the time ( $\sim 25$ cGPS sites were displaced by more than $10 \mathrm{~mm}$ ).

\subsubsection{Crustal anisotropy}

The seismic velocity of the crust is often dependent on the direction seismic rays are travelling. Shear wave splitting is one of the few means by which this directional dependence can be measured beneath the surface of the earth. When a shear wave enters a seismically anisotropic medium it is split into two orthogonally polarized quasi-shear waves with a fast and slow polarization (Crampin, 1981). The amount of anisotropy and the orientation of the fast polarization in the anisotropic layer(s) are represented by two splitting parameters; $\Phi$, the polariza- 


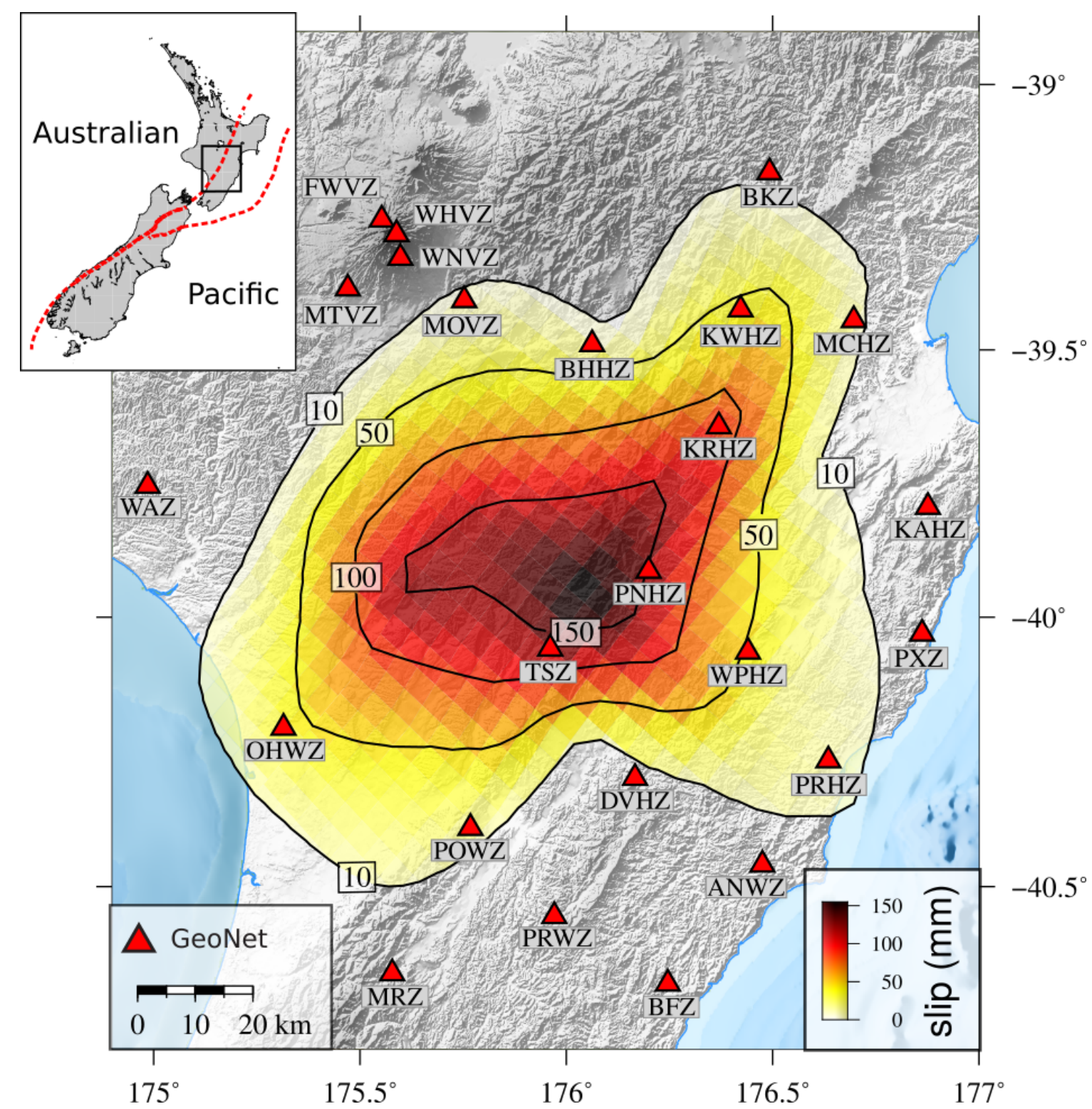

Figure 4.2: Slip contours for the 2010 Manawatu slow slip event [from Wallace et al. (2012b)]. Top: Locations of GeoNet stations (http:/ / www.geonet.org.nz) used in this study in central North Island, New Zealand. Total slip for the 2010 Manawatu slow slip event is overlain.

tion of the fast azimuth (or "fast azimuth") of the anisotropic material and $\delta t$, the delay time accrued during the travel through the anisotropic material (Silver and Chan, 1988). The delay time represents the difference between the arrival times of the fast and slow polarized waves and is dependent on the direction of travel and the path length within the anisotropic material (Crampin, 1981). If anisotropy changes along the path, the splitting will be most sensitive to the last layer traversed (e.g., Rumpker and Silver, 1998). 


\section{Distinguishing between stress and structurally-induced anisotropy}

Shear wave splitting is a powerful tool that can be used to help distinguish between stress and structure-induced anisotropy. In the crust, anisotropy is mainly caused by either pre-existing structural features, such as faults, dikes and mineral alignment (e.g., schists), or by the preferential closing of microcracks at particular orientations as a response to horizontal differential stresses (Babuska and Cara, 1991).

Several studies have previously investigated these two sources of anisotropy in the crust (e.g., Zinke and Zoback, 2000; Boness and Zoback, 2006; Johnson et al., 2011; Balfour et al., 2005). In the case of structurally-controlled anisotropy, the fast azimuth is usually oriented sub-parallel to the structural fabric (e.g., Savage, 1999; Zinke and Zoback, 2000; Cochran et al., 2006; Balfour et al., 2005). Large fault-parallel oriented anisotropy due to structural fabric have been observed at several fault zones, including the San Andreas Fault (i.e., Boness and Zoback, 2006; Cochran et al., 2006) and the North Anatolian Fault (Peng and Ben-Zion, 2004). Previous studies of seismic anisotropy across New Zealand have measured a dominant NE-SW fast azimuth around the northern region of South Island (e.g., Balfour et al., 2005; Karalliyadda and Savage, 2013) and across areas of North Island (e.g., Audoine et al., 2000; Evanzia et al., 2017; Illsley-Kemp et al., 2019) with some studies attributing this trend to pervasive shear fabrics associated with the plate-boundary deformation (i.e., Balfour et al., 2005), indicating a strong structural control.

Fluid-filled microcracks have been shown to be a dominant control of anisotropy in the upper crust (Crampin, 1981). microcracks may be inherently randomly oriented; however, differential horizontal stress will preferentially close the vertical cracks that are oriented perpendicular to the maximum horizontal compressive stress $\left(S_{H} \max ;\right.$ Nur, 1971). The aligned cracks will induce seismic anisotropy, such that the fast azimuth is parallel to $S_{H} \max$ (Crampin, 1981). The wave length of passing seismic waves is typically much longer than the size of the individual microcracks and so SWS typically reflects the dominant crack orientation. We consider these factors in the interpretation of the diverse distribution of anisotropy that we observe in the crust across central North Island. Cracks are also important in controlling the ratio of the speed of seismic compressional and shear waves, 
$V_{P} / V_{S}$. With higher volumes of liquid-filled cracks, S-wave velocities are reduced more than $P$ waves and hence the ratio increases (Nur, 1971).

Temporal variations in SWS fast azimuths have been previously observed at Mt. Ruapehu volcano in North Island (e.g., Miller and Savage, 2001; Gerst and Savage, 2004). There the observed SWS variations were explained by stress changing as a result of pore pressure changes in the magmatic system. Johnson et al. (2011) compared SWS results to focal mechanism inversions and fault orientations to identify areas around Mt. Ruapehu where SWS fast azimuths agree with stress estimations, as well as areas where fast azimuths align with fault orientations, and suggest both stress and structure-induced anisotropy around Mt. Ruapehu. We use a similar approach to reveal contributing sources of influence, by comparing our SWS measurements with both stress models and fault orientations. In the following section, we summarise previous studies of the stress field in New Zealand.

\subsubsection{Identifying principal stress orientations in the crust}

\section{Topography}

In a tectonic environment, the stress field may have a variety of sources. Gravitational stresses are a result of gravity acting on the crust and in the absence of other stresses acts to smooth any variations in topography, while tectonic stresses are driven by the motions of the plates relative to each other. There have been several approaches to calculating the stress field in North Island.

The weight of topography on the lithosphere in New Zealand significantly contributes to the stress field. This results in variations in gravitational potential energy (GPE) and differential stresses in the crust, also referred to as topographically induced stress (Flesch et al., 2007). Lamb (2015) and Hirschberg et al. (2019) used a thin sheet approximation of the lithosphere to solve stress balance equations for the vertically averaged deviatoric stress field. Using the method of Flesch et al. (2007), they assumed that the orientation of the total stress field is parallel to the strain rate field (i.e., same 'style'). Given that the lithospheric thickness ranges from $\sim 30 \mathrm{~km}$ in the Taupo region to around $150 \mathrm{~km}$ beneath the South Alps in South Island (Stern and Benson, 2011), Hirschberg et al. (2019) dealt with this problem by considering a range of models of the GPE field in New Zealand and 

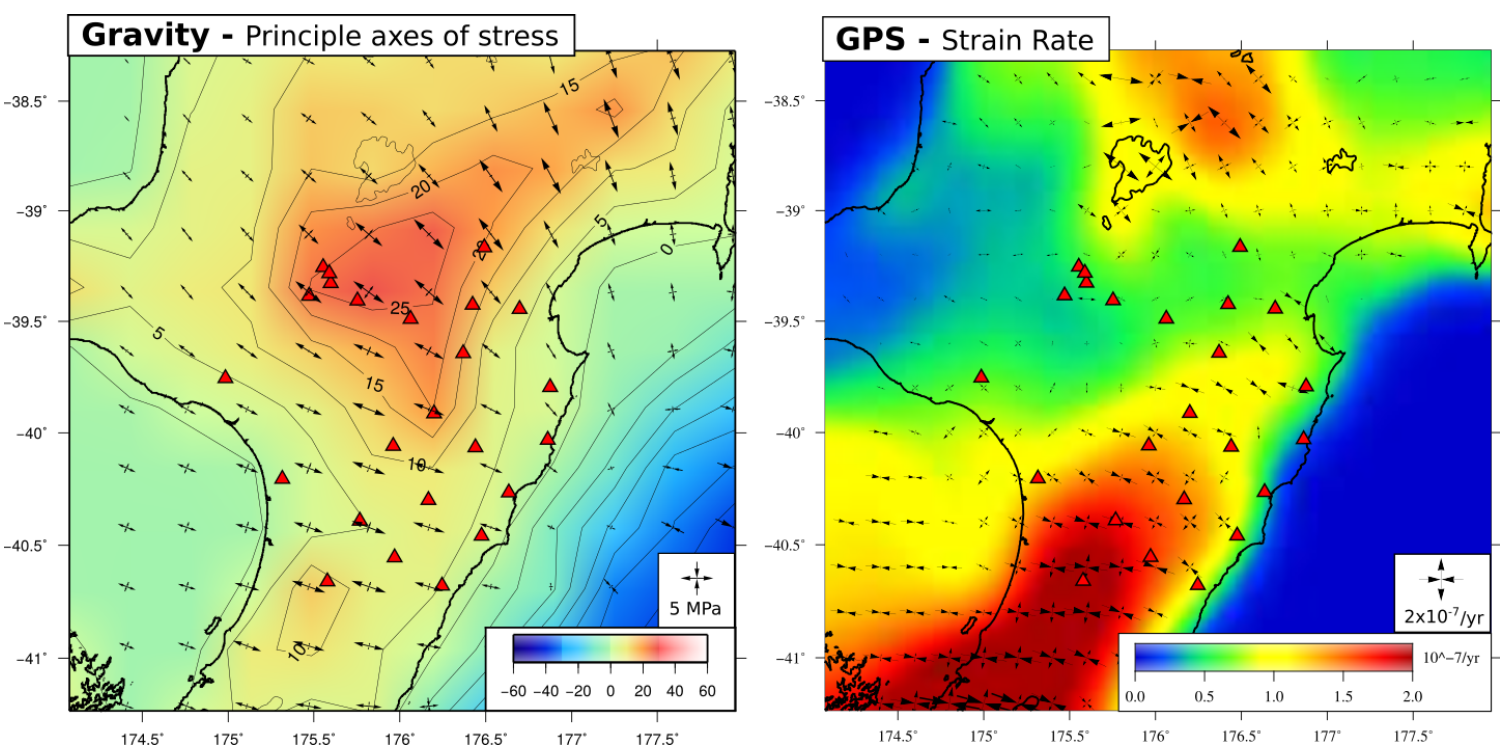

Figure 4.3: Extensional stresses are shown as outward pointing arrows and compressional stresses as inward pointing arrows. Left: Principal axes of deviatoric stress due to Gravitational Potential Energy (GPE) from Lamb (2015), colours and contours in MPa. Right: Principal strain rates, after Lamb (2015) and based on the observed velocity field from GPS data after Beavan et al. (2016). Colours indicate the strain rate magnitudes in $x 10^{-7} / y r$.

approximated the thin sheet model for layer thicknesses of 35 and $100 \mathrm{~km}$ using both topography and bathymetry. Using variations in the GPE per unit volume they calibrated the magnitudes of deviatoric stresses, assuming an isotropic viscosity. GPE varies from -10 to $30 \mathrm{MPa}$ in the onshore region of North Island, and decreases down to $-40 \mathrm{MPa}$ offshore (Figure 4.3). The most extensional gravitational stresses generally corresponded with areas of high topography and high GPE, such as Mount Ruapehu. The most compressional gravitational stresses generally corresponded with low topography, such as the Hikurangi trench, east of North Island.

\section{Strain rates from Quaternary and GPS-derived velocity fields}

Lamb (2015) calculated a 'long-term' Quaternary strain rate field based on the pattern and rate of active faulting in the New Zealand plate-boundary zone in the context of a network of triangles. Hirschberg et al. (2019) used the results of Lamb (2015) and also calculated the strain rate field using a high-resolution decadal GPSderived velocity model of Beavan et al. (2016). This was gridded by constructing the most equilateral triangular network (i.e., Delauney network) of GPS sites and calculating velocities at internal grid points by assuming a constant velocity 
gradient within each triangle. Both Lamb (2015) and Hirschberg et al. (2019) assumed a bulk viscous rheology for the thin sheet and so the principal stress axes are parallel to the principal strain rate axes such that the minimum strain rate (i.e., most negative) will correspond to the maximum horizontal compressive stress axis (Figure 4.3). We considered that the GPS-derived and Quaternary stress orientations are very similar (Hirschberg et al., 2019), and in this study we focused on the GPS-derived orientations because they provided a much higher resolution. GPS-derived velocities were well constrained for onshore North Island due to a continuous dense network of GeoNet GPS sites. In southern North Island, GPSderived maximum compressive stresses were oriented mainly E-W, likely due to elastic strain accumulation as a result of locking between the plates in this region (e.g., Hirschberg et al., 2019).

\section{Tectonic stress and relationship to faulting}

It is commonly assumed that faulting follows the Andersonian model of stress (Anderson, 1905). In this model, it is assumed that the three principal stresses typically consist of one vertical $\left(S_{V}\right)$ and two horizontal components $\left(S_{H} \max\right.$ and $\left.S_{h} \min \right)$. There are three commonly defined regimes of stress for the active faults in North Island, each associated with different fault orientations, (a) normal fault regime, with $S_{H} \max$ sub-parallel/parallel to the strike of the fault, (b) reverse fault regime, with $S_{H} \max$ essentially perpendicular to the strike of the fault, and

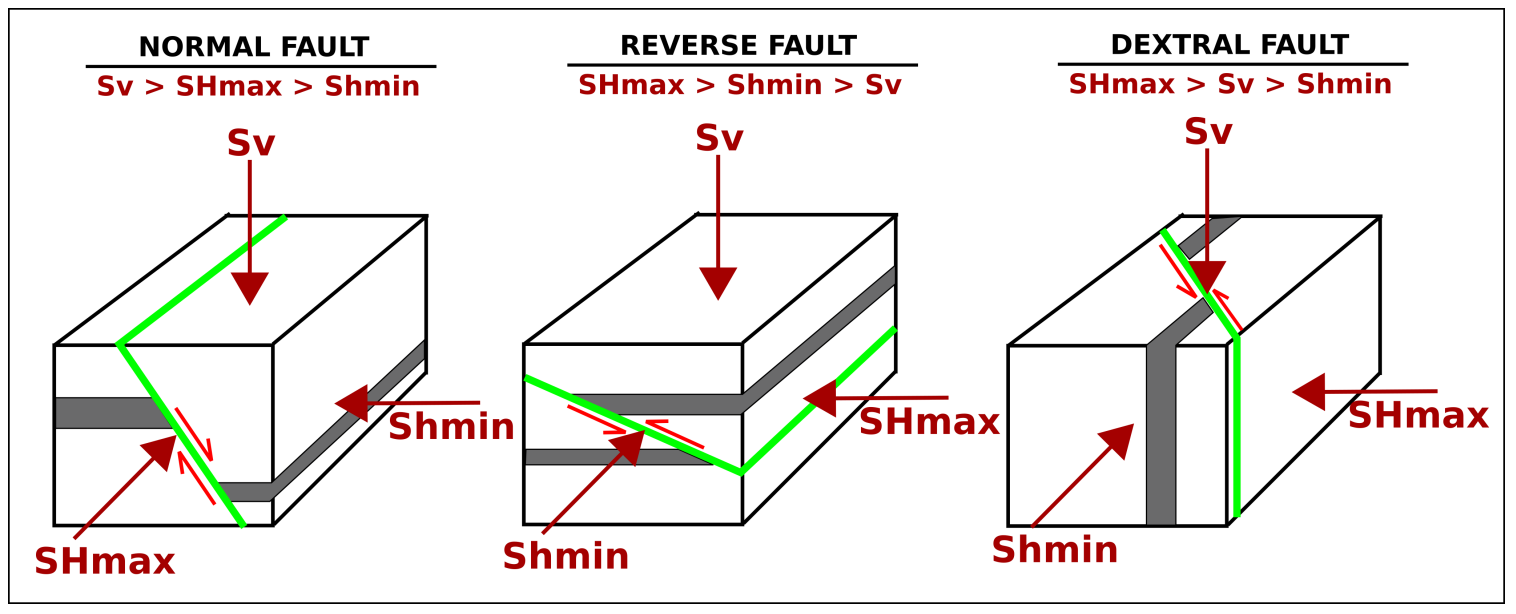

Figure 4.4: Common faulting kinematics relative to principal stress directions in the Earth's crust. The type of faulting is controlled by the relative magnitude of the stresses. $S_{V}$, vertical stress; $S_{H} \max$, maximum horizontal stress; $S_{h} \min$, minimum horizontal stress (Anderson, 1905). 


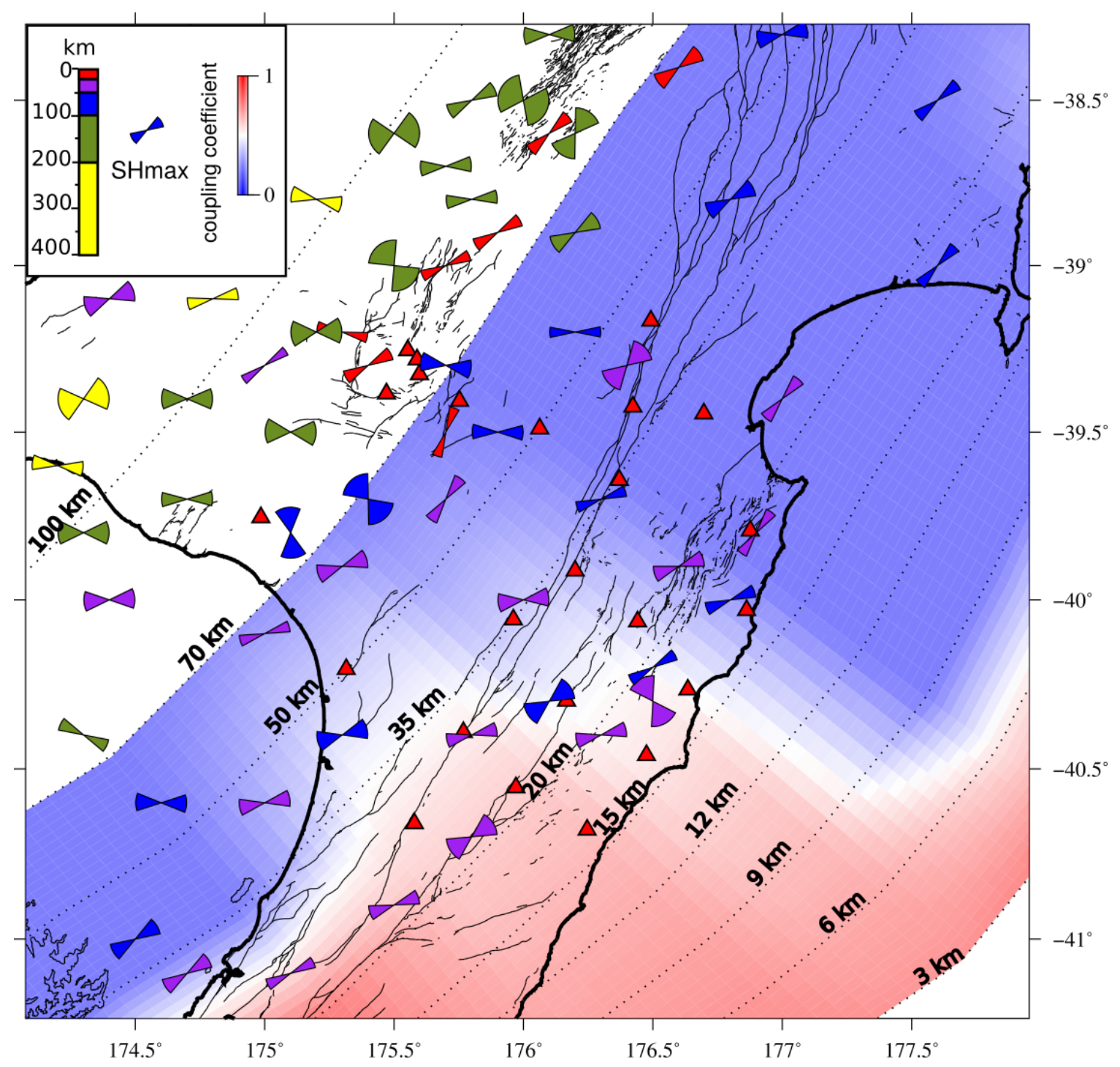

Figure 4.5: $S_{H} \max$ directions derived from focal mechanisms are overlain and coloured by their depth (Townend et al., 2012). Subduction interface and interseismic coupling on the plate interface for the Hikurangi subduction zone (Wallace et al., 2012b). The coupling coefficient ranges from 0-1 (freely slipping to fully locked). Red triangles mark locations of the GeoNet stations used in this study. Black lines mark active fault traces from Langridge et al. (2016) and dotted lines showing the depth contours of the plate interface are from Williams et al. (2013).

(c) dextral fault regime, with $S_{H} \max$ between $30^{\circ}-60^{\circ}$ to the strike of the fault (Figure 4.4; Anderson, 1905). Many of the major active faults in the North Island are oriented in a NE-SW direction, sub-parallel to the NIDFB (Figure 4.1; Langridge et al., 2016). While the NIDFB is comprised of faults with a dominantly dextral slip regime, large areas of reverse faulting occur across the eastern parts of the Wanganui Basin and southwest of Napier (Figure 4.1). Intense normal faulting accommodates extension in the TVZ. 
The orientation of the stress field across New Zealand has been determined from the inversion of earthquake focal mechanisms by Townend et al. (2012) (shown in Figure 4.5). Using a clustering algorithm, Townend et al. (2012) defined clusters of focal mechanisms that are used to calculate $S_{H} \max$ directions across North Island. The orientation of $S_{H} \max$ along the NIDFB is NE-SW (parallel to the Hikurangi Margin) in northern North Island, with more ENE-WSW directions across southern North Island. $S_{H} \max$ directions in the Wanganui Basin tend to be oriented closer to E-W, with greater variations in directions near Mt. Ruapehu.

\subsection{Data and methods}

The GeoNet permanent land-based seismic stations used in this study consisted of 24 instruments of which 8 were broadband and 16 were short-period seismometers. Using GeoNet's earthquake locations we analyzed 20,832 local earthquakes compiled from June 2009 to October 2012, within our spatial limits (174.5 $5^{\circ}$ to $177.5^{\circ}$ and $-38.5^{\circ}$ to $-41^{\circ}$ ) for depths $<100 \mathrm{~km}$; magnitudes ranging from Mw 0-5.1 (Figure 4.6).

83,013 shear wave arrivals were automatically picked across 24 stations using the technique described in Section 4.3.1. We then applied the Multiple Filter Automatic Splitting Technique (MFAST; Savage et al., 2010b) on the associated catalog of waveforms. By applying this technique to our data we produced a total of 42,423 A and B grade measurements. A description of the MFAST technique and the chosen parameters can be found in section 4.3.2.

\subsubsection{Local phase arrival picking}

We used an automatic picking technique (SPICKERC) to pick S-wave arrival times on 20,832 local earthquakes across 24 stations. S-phase arrivals were automatically picked using the SPICKERC method by Diehl et al. (2009) and adapted by Castellazzi et al. (2015) on seismograms from GeoNet containing an origin time and P pick. SPICKERC combines three different detection and picking methods: STA/LTA, or short term average versus long term average, as described by Allen (1978), polarization detection based on the approach by Cichowicz (1993), and autoregressive picking using the Akaike Information Criterion (AR-AIC) as described by Leonard and Kennett (1999). The final pick is a weighted mean of all 


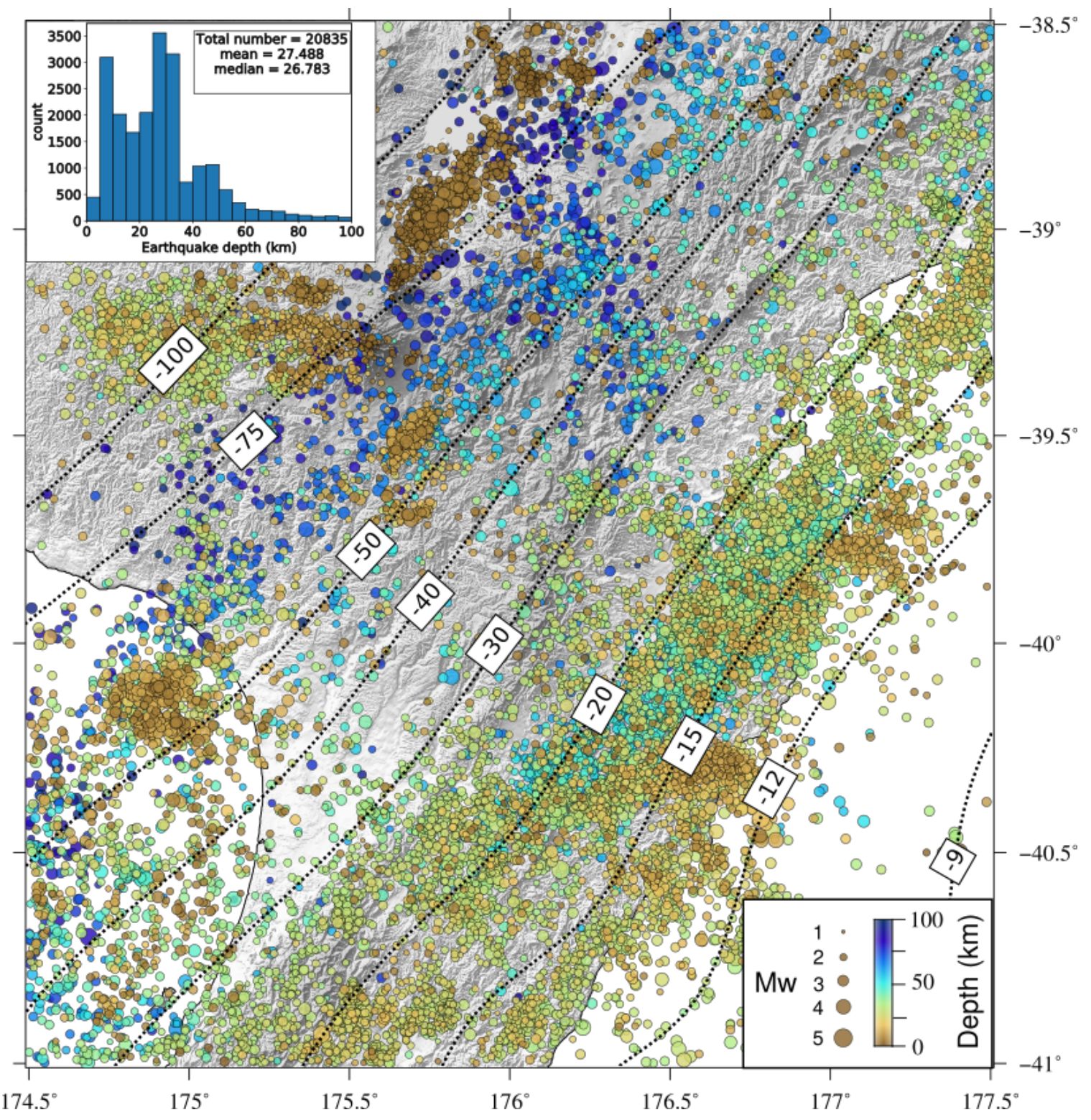

Figure 4.6: GeoNet earthquake locations in our study area, and above $100 \mathrm{~km}$ depth between June 2009 to October 2012 and earthquake magnitudes (Mw) range from 0 to 5.1 (http://www.geonet.org.nz). The dotted contours of the plate interface are from Williams et al. (2013).

three methods with a grade based on the error estimate and the signal to noise ratio around the pick (Castellazzi et al., 2015).

\subsubsection{Automatic Shear Wave Splitting (MFAST)}

The splitting was analysed using Multiple Filter Automatic Splitting Technique (MFAST; Savage et al., 2010b). MFASTv2.2 executes the following steps: (1) apply multiple band-pass filters on the $S$ arrival waveform and calculate the best prod- 
uct of signal-to-noise ratio and bandwidth (SNR); (2) apply the SWS algorithm of Silver and Chan (1991) to perform a grid search over the fast azimuth and delay time parameter space and determine the best parameters that correct for splitting, measured by the minimum of the smallest eigenvalue of the corrected particle motion; (3) automatically analyse multiple measurement windows, whose length is based on the period of the wave and whose starting values depend on the S arrival, using the Silver and Chan (1991) analysis technique and determine the best window using the cluster analysis technique of Teanby et al. (2004b); (4) assign a grade from A to D, based on the SNR, uncertainty, distinctiveness of the cluster, and whether the measurement is a null (Savage et al., 2010b).

The incidence angle for each event-station pair was determined using the Taup Toolkit (Crotwell et al., 1999) with a 1-D velocity model created for the study area (Appendix C, Table C.1) from the 3-D model by Eberhart-Phillips et al. (2010). The 1-D model was created by averaging all velocities across the spatial extent of our study region. Because rays with incidence angles greater than 35 degrees from vertical are outside the shear wave window, they were excluded from the analyses (Nuttli, 1961). We used both A and B grade measurements for our analyses.

\subsection{3 $\quad \mathrm{V}_{\mathrm{P}} / \mathrm{V}_{\mathrm{S}}$}

We calculated the ratio of $\mathrm{P}$ - to S-velocities at each station for all earthquakes in the catalog that contained a $\mathrm{P}$ and $\mathrm{S}$ phase pick. Following the approach by Wadati and Oki (1933) we calculated an average ratio of P- to S-velocity $\left(V_{P} / V_{S}\right)$ along the ray path from the arrival times at each station:

$$
V_{P} / V_{S}=t_{S} / t_{P}
$$

where $t_{S}=T_{S}-T_{O}$ and $t_{P}=T_{P}-T_{O}$, with $T_{S}$ and $T_{P}$ being the arrival times of the $\mathrm{S}$ - and $\mathrm{P}$ - wave, respectively, and $T_{O}$ is the origin time of the earthquake.

\subsubsection{Spatial averaging of fast azimuths}

To calculate spatial averages of fast polarization azimuths, $(\Phi)$, we used the Tomography Estimation and Shear-wave-splitting Spatial Average (TESSA) package by Johnson et al. (2011). TESSA works by gridding the study area and then assigning the fast polarization azimuths for each earthquake to every spatial grid-block 
through which the ray passed. To account for potential overprinting of $\Phi$ azimuths later in the path, the individual $\Phi$ values within each grid-block were weighted inversely proportional to the square of the distance from the station. We assigned a minimum block size of $6 \mathrm{~km}$, which resulted in 20-120 rays passing through each grid-block (Figure 4.7). Fast polarization azimuths were not plotted for blocks with average values that had a standard deviation greater than $30^{\circ}$ and standard error larger than $10^{\circ}$ and for the areas with sparse raypath coverage (less than 20 rays), which is controlled by station and earthquake distribution (Figure 4.7).

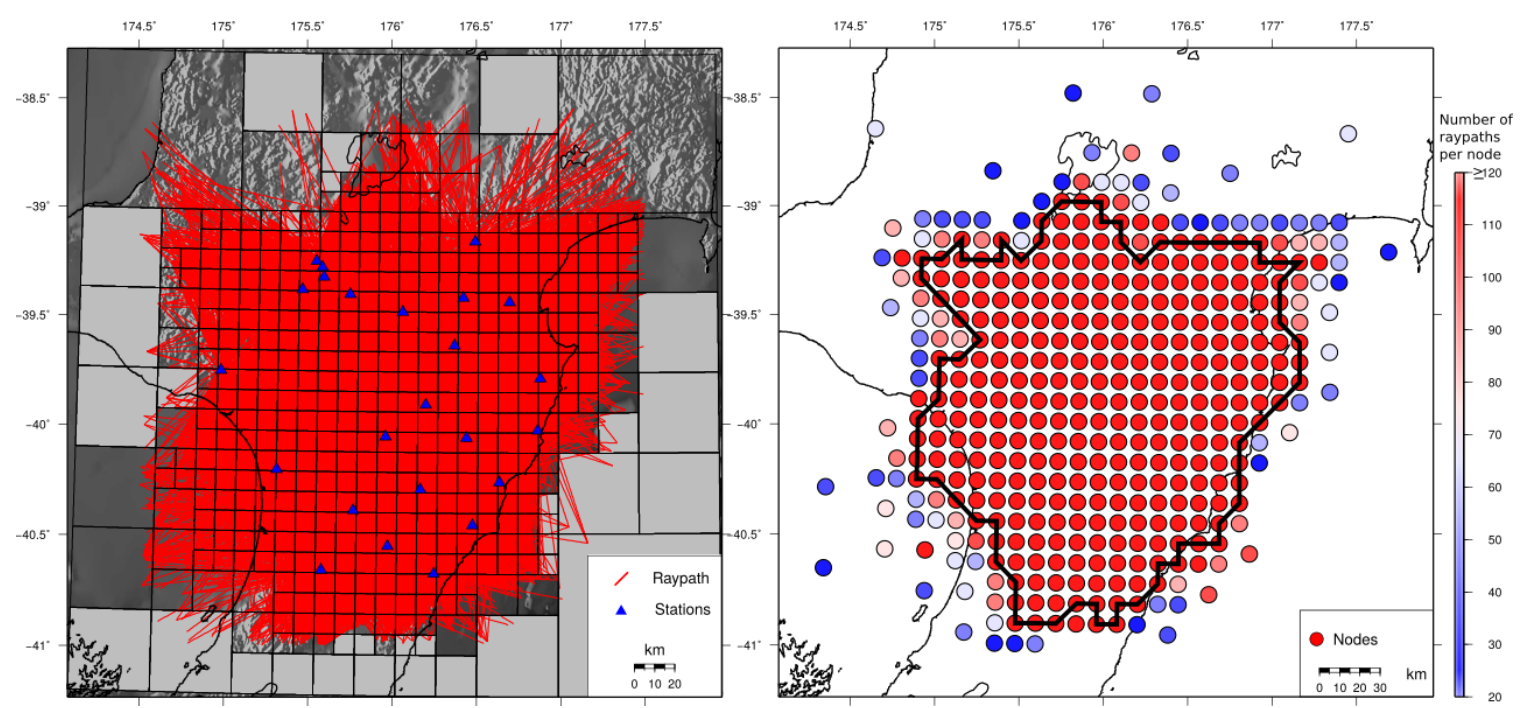

Figure 4.7: Left: Raypath plot using quadtree gridding following the method described by Johnson et al. (2011). Blue triangles mark locations of seismic stations and red lines show raypaths. A minimum box size of $6 \mathrm{~km}$ is chosen, containing between 20 and 120 raypaths passing through each box. Gray shaded boxes did not contain sufficient raypath coverage and were not used. Right: Plot of nodes coloured by the number of earthquake raypaths. The black contour marks nodes with 120 or more rays and indicates the area of greatest confidence.

\subsubsection{Quantitative comparison of fast azimuth measurements}

We used the Circular Statistics Toolbox in Matlab ${ }^{T M}$ to quantitatively compare the spatially averaged fast polarization azimuths to GPS-derived maximum compression directions, $S_{H \max }^{\text {grav }}$ from our gravitational stress analyses, and to fault orientations. To accomplish this task, we averaged the fast azimuths within each gridblock of the GPS and gravitational stress analyses and compared the two angles. We compared our spatially averaged fast azimuths to nearby active faults from Langridge et al. (2016) by averaging all fault orientations within a $6 \mathrm{~km}$ radius of each fast azimuth. The two angles were compared by taking the absolute value of 
the cosine of the difference between the angles. The resulting values range from 0 to 1 , where 0 represents perpendicular and 1 represents parallel azimuths between the two angles. We contoured these using the GMT function 'surface' (Wessel et al., 2013). The grid-blocks which did not contain a comparative direction were masked.

\section{GPS}

We used strain rates derived by Hirschberg et al. (2019) from the onshore GPSderived velocity field of Beavan et al. (2016) to compare to our SWS fast azimuths. The GPS-derived velocities were gridded at a $20 \mathrm{~km}$ scale by constructing the most equilateral triangular network of GPS sites (Hirschberg et al., 2019). The comparison analysis searched for, and averaged all spatially averaged fast azimuths within the grid-blocks of each GPS-derived $S_{H \max }$ measurement.

\section{Gravity}

We compared our SWS fast azimuths to the topographic stress model of Hirschberg et al. (2019). That model was based on the method by Flesch et al. (2001), given the density of the crustal rocks, crustal thickness and topography and bathymetry. In general, the Flesch et al. (2001) method involves solving force balance equations for an isotropic viscous medium, subject to a mean vertical stress and a horizontal boundary condition of zero deviatoric stress. We implemented this method in our study using finite difference methods (Hirschberg et al., 2019) in Matlab ${ }^{T M}$. We used the topography and bathymetry of North Island to calculate the $S_{H \max }^{\text {grav }}$ directions and GPE, averaged over the top $\sim 30 \mathrm{~km}$ of the crust, with an average density of $2.67 \mathrm{~g} / \mathrm{cm}^{3}$. We focused on the topographically induced $S_{H \max }$ orientations, referred to as $S_{H \max }^{\text {grav }}$, to compare to our SWS fast azimuths. $S_{H \max }^{\text {grav }}$ directions were gridded at a $30 \mathrm{~km}$ scale. The stress field reflects variations in gravitational forces at a length scale of tens of kilometers and thus is characteristic of these stresses over a comparable thickness (Hirschberg et al., 2019). The $S_{\text {Hmax }}^{\text {grav }}$ at each grid point was compared to the average fast azimuth within each grid-block.

\section{Fault orientations}

We compared our spatially averaged fast azimuths to the orientations of nearby active faults from Langridge et al. (2016). Fault geometries were available in tabu- 
lated form in terms of segments with beginning and end points. Fault orientations were determined by calculating the angle (with respect to North) of each fault segment and plotting the angle at the mid-point of each segment. We averaged all the fault orientations within a chosen search radius of $6 \mathrm{~km}$ of each fast azimuth measurement.

Additionally, we determined the fault-derived $S_{H \max }$ by adding $90^{\circ}$ to all reverse faults, $45^{\circ}$ to all dextral faults, and left normal faults unchanged, as the expected $S_{H \max }$ for normal faults is parallel to the strike of the faults based on the Andersonian model for stress on faults (Figure 4.4; Anderson, 1905). We then compared our spatially averaged fast azimuths to the fault-derived $S_{H \max }$.

\subsubsection{Spatial averaging of delay times}

We calculated spatial averages of delay times using a similar method to the spatial averaging of fast azimuths. Delay times are path-averaged properties and, unlike SWS fast azimuths, they are not necessarily most strongly influenced by properties in close proximity to the station where they are recorded. Because the delay time is often a cumulative property, we spatially normalized the delay times for each event-station pair: the station-event distance for each raypath was divided by a node spacing of $6 \mathrm{~km}$, and the delay time value was then divided equally by the number of nodes for each raypath. We gridded the study area and assigned the spatially normalized delay time values for each earthquake to every spatial grid-block through which the ray passed. We assigned a minimum block size of $6 \mathrm{~km}$ which resulted in 20-120 rays passing through each grid-block (Figure 4.7). Delay times were not plotted for blocks with sparse raypath coverage (less than 20 rays), which is controlled by station and earthquake distribution. The delay times within each grid-block were averaged and then contoured using the GMT function 'surface' (Wessel et al., 2013). The areas of confidence were defined by plotting only the grid-nodes which contained more than 20 rays and by masking areas for which data coverage was poor (see bold contour in Figure 4.7).

\subsubsection{Temporal analysis}

We searched for changes in time during the 18-month long 2010-2011 Manawatu slow slip event in our SWS measurements as well as $V_{P} / V_{S}$. To look for temporal 
changes at each station, the moving median values of all $V_{P} / V_{S}$ and SWS delay time measurements were determined. To calculate the moving median of $n$ values $\left(d_{i}\right)$, where $i$ is between zero and $n$, the values were ranked and the median was represented by the middle value $\left(d_{\frac{n}{2}}\right)$. The $95 \%$ confidence limits of the median were calculated using the method of Altman (Altman et al., 2000). In this method, the lower $95 \%$ confidence limit is the ranked value $d_{l}$, where $l$ is given by:

$$
l=\left\lfloor\frac{n}{2}-\frac{1.96 \sqrt{n}}{2}\right\rceil
$$

and the upper $95 \%$ confidence limit is the ranked value $d_{u}$, where $u$ given by:

$$
u=\left\lfloor 1+\frac{n}{2}+\frac{1.96 \sqrt{n}}{2}\right\rceil .
$$

Generally, the median is more robust than the mean and is less sensitive to outliers, but it is more difficult to apply to azimuthal values (Altman et al., 2000). Therefore, values of fast polarization azimuths for the moving windows were calculated using a circular mean. These circular moving means were calculated using the 'circular' function (https://CRAN.R-project.org/package=circular) in R. 95\% confidence intervals of the mean were calculated by bootstrapping using the 'mle.vonmises.bootstrap.ci' function (https:/ /CRAN.R-project.org/package=circular) in $\mathrm{R}$ and re-sampling the dataset 9999 times.

\subsection{Results}

\subsubsection{Temporal analysis}

The three year time frame of our earthquake dataset allowed us to search for temporal variations during the 2010 Manawatu slow slip event. We searched for temporal variations in $V_{P} / V_{S}$, delay time, and fast azimuths at individual stations using the technique described in section 4.3.7. After examining several window lengths for temporal averaging, we chose a 40-day window, with a window step of 1 day. The 40-day time window was narrow enough to search for changes within the year-long SSE without excessive smoothing and wide enough to provide acceptable confidence intervals. The 40-day moving windows were plotted at the center of the window, so any observed changes could start 20 days earlier or later. Our results did not show significant variations at individual stations which 
could be interpreted within the calculated 95\% confidence intervals. An example is shown in Figure 4.8. Individual station plots can be found in Appendix C, Figure C.1.

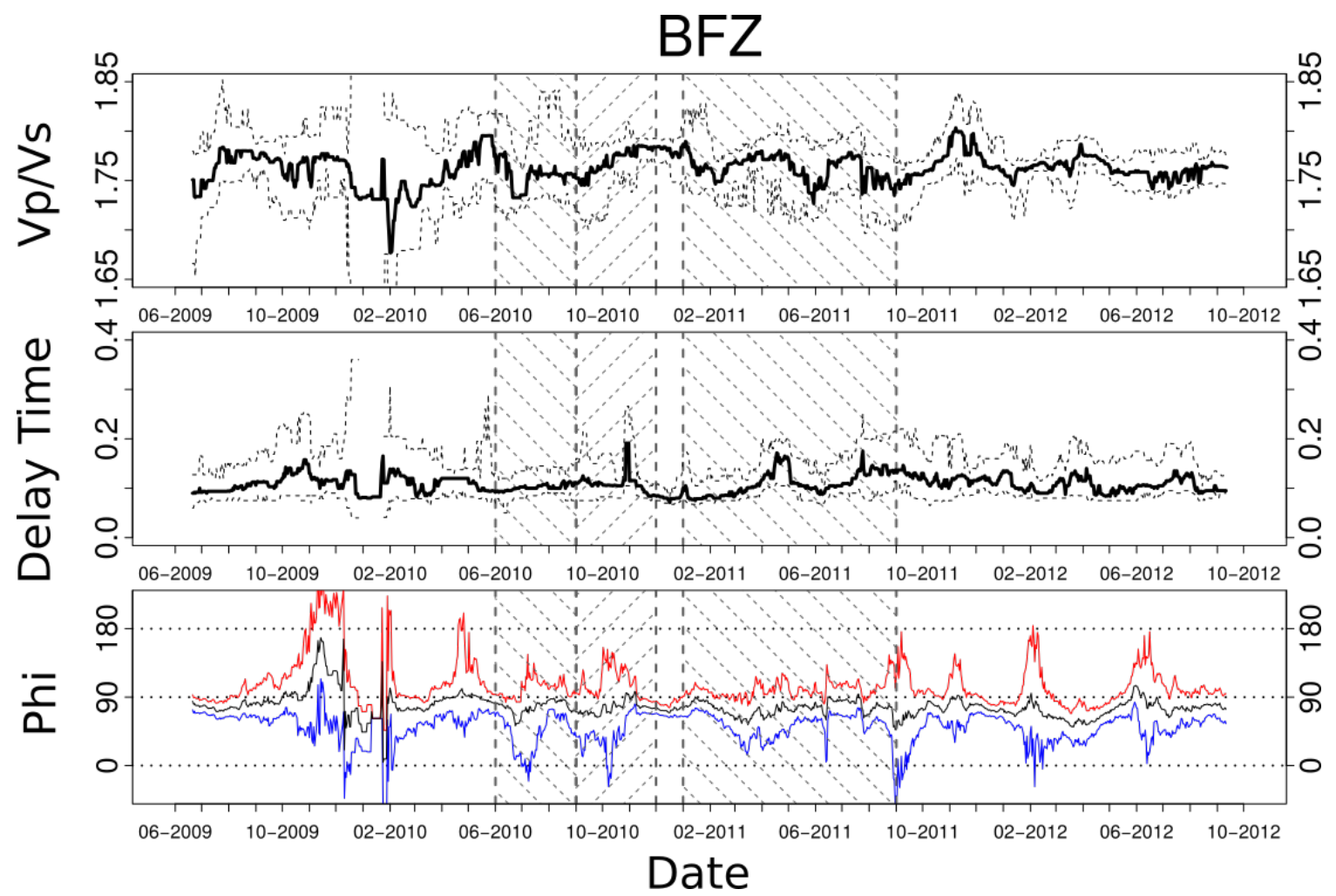

Figure 4.8: Example results for shear wave splitting and Vp/Vs versus time for station BFZ. Vp/Vs and Delay time: Black line is a median of 40 day moving windows with $95 \%$ confidence intervals. The 3 stages of the 2010 Manawatu SSE are shaded with diagonal dotted lines. Phi: Fast polarization directions. Black line is a circular mean of 40 day moving windows with $95 \%$ confidence intervals.

In subsequent analysis, we isolated individual clusters of earthquakes in order to test for temporal variations from earthquakes from similar back-azimuths. However, the two largest clusters chosen only resulted in $\sim 200$ A and B grade measurements per station, resulting in large gaps in the data with large confidence intervals and we are unable to observe any significant variations from these clusters. GeoNet GPS sites are located in close proximity to most seismic stations. We compared our measured temporal variations to cGPS displacements in search of similar trends (Appendix C, Figure C.2). However, we do not find any correlation between the compared plots. 


\subsubsection{Individual station averaged fast polarization azimuths}

We made a total of 42,423 A and B grade measurements across 24 GeoNet stations in North Island. We observed significant variations in average station orientations (Figure 4.9 and Table 4.1). Across the eastern North Island fast azimuths were

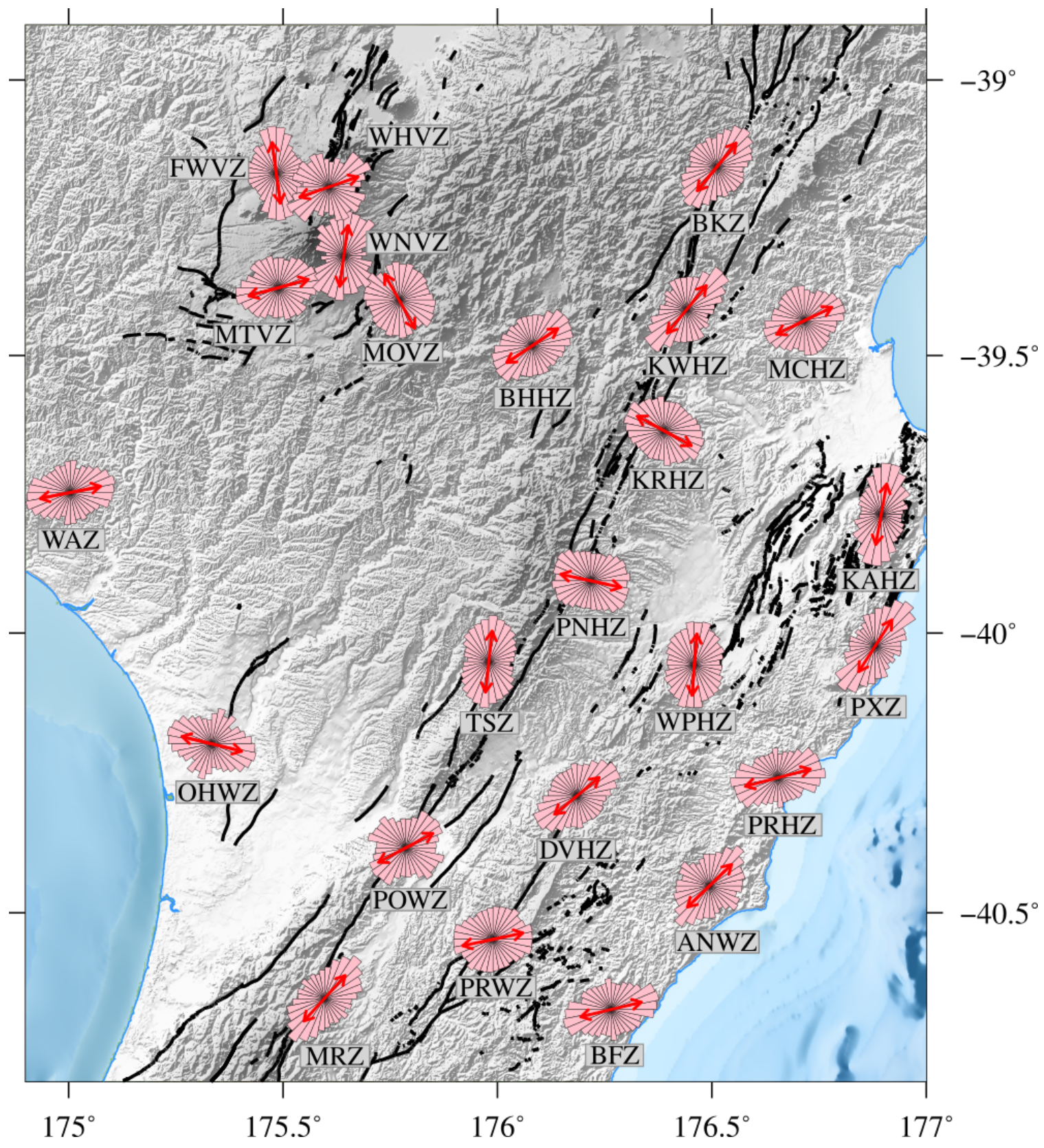

Figure 4.9: Rose diagrams of fast polarization azimuths calculated using MFAST (Savage et al., 2010b). Rose diagrams are plotted on the stations at which measurements were made. The length of the sectors are proportional to the square root of the number of measurements in each sector. The red arrows show the circular mean azimuths. Dashed black lines mark active faults from Langridge et al. (2016). 
Table 4.1: Average $\Phi, V_{P} / V_{S}$, and delay time for individual Stations

\begin{tabular}{|l|c|c|c|c|c|c|c|c} 
Station & $\mathrm{N}$ & $\mu(\phi)$ & $95^{\circ} \% \mathrm{CIs}$ & Median $\delta t$ & $95 \%$ CIs & Median $V_{P} / V_{S}$ & \multicolumn{2}{c}{$95^{\circ} \mathrm{CIs}$} \\
\hline ANWZ & 1505 & $45.9^{\circ}$ & {$\left[41.1^{\circ}, 50.7^{\circ}\right]$} & $0.25 \mathrm{~s}$ & {$[0.24 \mathrm{~s}, 0.26 \mathrm{~s}]$} & 1.80 & $1.80,1.81]$ \\
BFZ & 1418 & $76.4^{\circ}$ & {$\left[72.9^{\circ}, 79.7^{\circ}\right]$} & $0.12 \mathrm{~s}$ & {$[0.11 \mathrm{~s}, 0.13 \mathrm{~s}]$} & 1.77 & {$[1.76,1.77]$} \\
BHHZ & 2789 & $55.8^{\circ}$ & {$\left[52.7^{\circ}, 58.8^{\circ}\right]$} & $0.18 \mathrm{~s}$ & {$[0.17 \mathrm{~s}, 0.19 \mathrm{~s}]$} & 1.70 & {$[1.70,1.71]$} \\
BKZ & 2548 & $39.7^{\circ}$ & {$\left[36.7^{\circ}, 42.6^{\circ}\right]$} & $0.15 \mathrm{~s}$ & {$[0.15 \mathrm{~s}, 0.16 \mathrm{~s}]$} & 1.73 & {$[1.73,1.73]$} \\
DVHZ & 2680 & $50.5^{\circ}$ & {$\left[47.9^{\circ}, 53.2^{\circ}\right]$} & $0.18 \mathrm{~s}$ & {$[0.18 \mathrm{~s}, 0.19 \mathrm{~s}]$} & 1.73 & {$[1.73,1.74]$} \\
FWVZ & 992 & $-7.6^{\circ}$ & {$\left[-13.9^{\circ},-1.3^{\circ}\right]$} & $0.14 \mathrm{~s}$ & {$[0.12 \mathrm{~s}, 0.15 \mathrm{~s}]$} & 1.71 & {$[1.71,1.72]$} \\
KAHZ & 1435 & $10.2^{\circ}$ & {$\left[7.9^{\circ}, 12.6^{\circ}\right]$} & $0.22 \mathrm{~s}$ & {$[0.21 \mathrm{~s}, 0.23 \mathrm{~s}]$} & 1.82 & {$[1.81,1.82]$} \\
KRHZ & 3475 & $-61.1^{\circ}$ & {$\left[-65.2^{\circ},-57.1^{\circ}\right]$} & $0.17 \mathrm{~s}$ & {$[0.16 \mathrm{~s}, 0.17 \mathrm{~s}]$} & 1.73 &
\end{tabular}


fairly consistently aligned N-S to NE-SW, sub-parallel to the trench convergence direction $\left(30^{\circ}-60^{\circ}\right)$ and the NIDFB $(\sim \mathrm{NE})$. In striking contrast to the surrounding NE trend, stations KRHZ and PNHZ, located along the NIDFB, exhibit azimuths with almost perpendicular, NW-SE directions. Located in the Wanganui Basin, stations WAZ and OHWZ have close to E-W azimuths. Stations located closest to Mt. Ruapehu show significant variations in fast azimuths. Stations located on the western and eastern sides of Ruapehu, FWVZ and WNVZ, respectively, are oriented N-S, while stations located on the northern and southern sides, WHVZ and MTVZ, respectively, are oriented closer to E-W. Station MOVZ, located southeast of Ruapehu, has a NW-SE mean azimuth.

\subsubsection{Spatial averaging of fast polarization azimuths}

The advantageously broad distribution of GeoNet stations and earthquakes provided for almost complete ray-path coverage across central North Island (Figure 4.7). Using the spatial averaging technique explained in section 4.3.4, we observe a complex distribution of fast polarization azimuths across North Island (Figure 4.10). Along the NIDFB, azimuths are dominantly NE-SW, trench-parallel.

In order to examine the spatially averaged fast azimuths in greater detail we define four areas of interest (Boxes 1-4; Figure 4.10). Box 1 marks the area of the NIDFB where directions are generally NE-SW. Boxes 2-4 mark areas where fast azimuths exhibit deviations from this general NE-SW trend (Boxes 1-4; Figure 4.10). Box 2 is located within Box 1 and highlights an area of E-W trending azimuths, a significant change from the NE-SW directions observed across the rest of Box 1. Box 3, across the Wanganui Basin in western North Island, also exhibits dominantly E-W azimuths. Finally, Box 4 highlights an area of varying fast polarization azimuths around Mount Ruapehu. These chosen areas are used to conduct statistical tests which compare our observed fast azimuths to other stress models, as well as to fault orientations. Since Box 2 is located within Box 1, for further analysis we exclude measurements in Box 2 from our measurements in Box 1.

\subsubsection{Depth extent of anisotropy}

Cracks tend to close completely with depth due to lithostatic pressure, and as a result, most studies of upper crustal anisotropy find little change in splitting with 


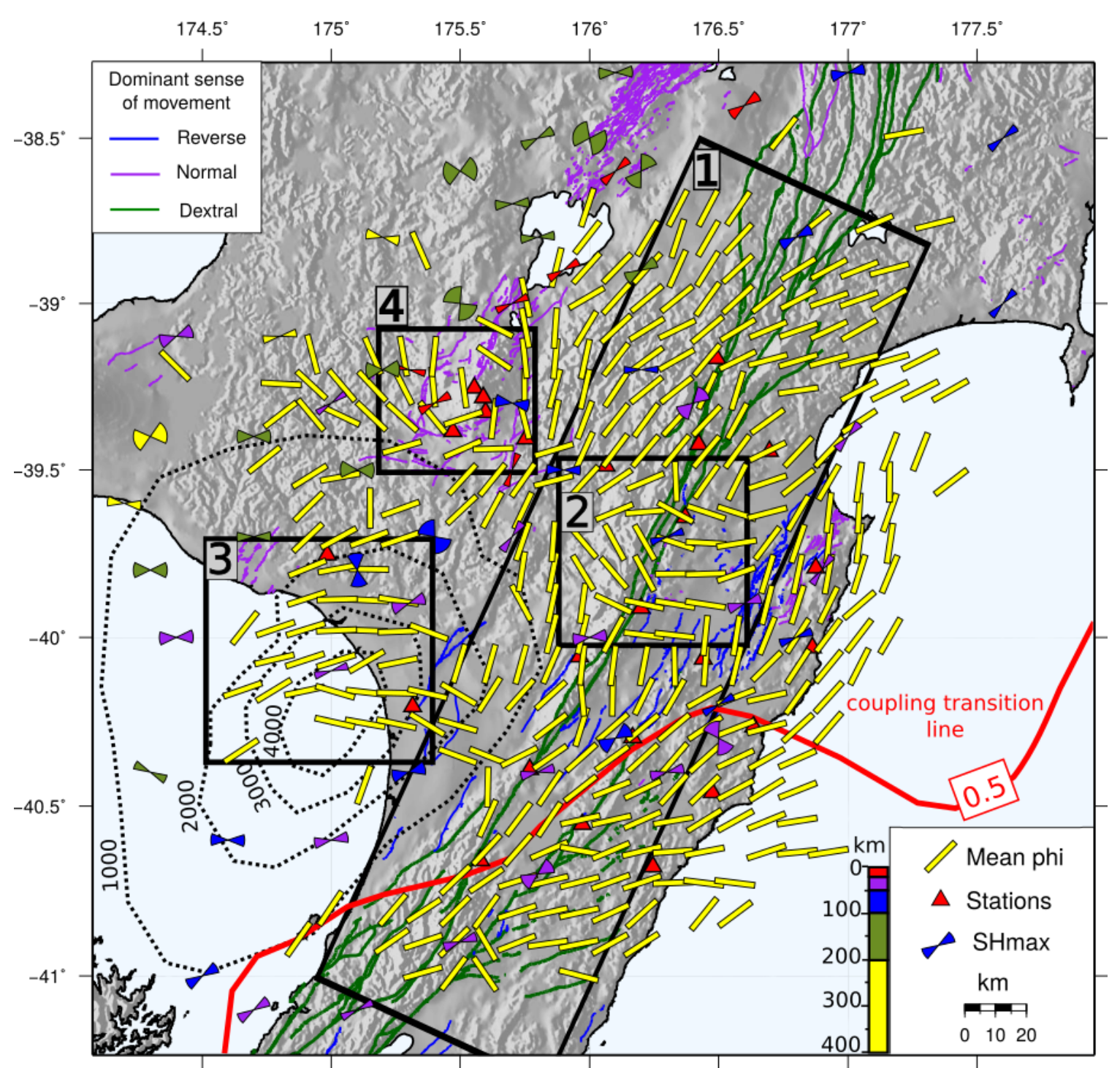

Figure 4.10: Spatial averages of SWS fast polarization azimuths from TESSA (Johnson et al., 2011) using the entire catalog of earthquakes. Yellow bars show average fast polarization azimuths for each block plotted in the center of the grid block. Fast polarization azimuths are not plotted for blocks with average values that have a standard deviation greater than $30^{\circ}$ and standard error larger than $10^{\circ}$. Also illustrated is the $\Phi=0.5$ subduction thrust coupling coefficient contour (red; Wallace et al., 2012b). Boxes 2-4 mark areas where fast polarization azimuths change from the general NE-SW trend in Box 1. Bow-tie symbols are $S_{H} \max$ orientations obtained by Townend et al. (2012) and are coloured by depth. Red triangles mark locations of seismic stations used in this study. Dotted contours are isopachs (interval is $1 \mathrm{~km}$ ) of Pliocene-Pleistocene sedimentary rocks in the Wanganui Basin [after Harmsen (1985)].

depth, suggesting that the top few kilometers are most important for splitting measurements (e.g., Gledhill, 1993). In order to search for variations in our SWS fast azimuths with depth we compare two sub-catalogs of spatially averaged fast azimuths consisting of earthquakes between 0-30 km and 30-100 km (Appendix C, 
Figure C.3). We chose a $30 \mathrm{~km}$ depth to separate our measurements based on previous studies which have observed a crustal thickness across the Hikurangi Plateau ranging between 10-35 km (Wood and Davy, 1994; Tozer et al., 2017; Reyners et al., 2011). However, we note that there is a large variation in the depth of the subduction interface across our study region. We analyze 22,087 measurements from 12,847 earthquakes between 0-30 km depth and 20,336 measurements from 7,985 earthquakes between $30-100 \mathrm{~km}$. We find no significant differences in our spatially averaged fast azimuths between the two subsets of earthquakes, suggesting that our fast azimuths do not vary significantly with depth or that they are controlled by anisotropy at the top layer. Additionally, we analysed the dependence of delay times and fast azimuths on hypocentral depth at individual stations. We present an example from station ANWZ in Figure 4.11. Results from all the stations can be found in Appendix C, Figure C.4. We do not observe any discernible changes in delay time or fast azimuth with increasing depth. Likewise, previous SWS studies in New Zealand have also observed no correlation between delay time and depth over similar depth lengths (e.g., Audoine et al., 2000; Balfour et al., 2005; Johnson et al., 2011), suggesting that we are mainly measuring anisotropy in the uppermost few kilometers of the crust.

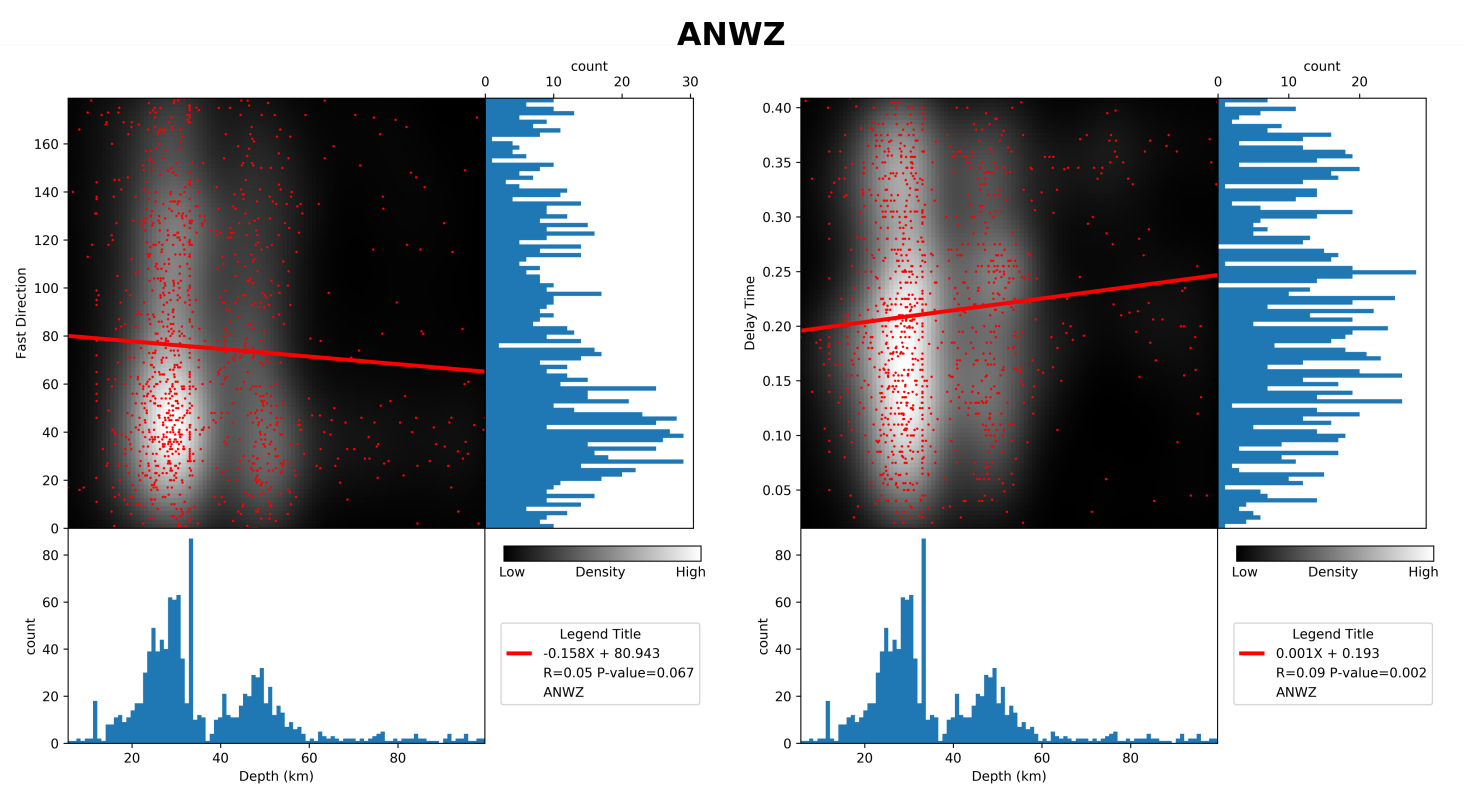

Figure 4.11: Left column: Fast polarization azimuths versus depth. Individual measurements are coloured by their event-station back-azimuth. $\mathrm{X}$ and $\mathrm{Y}$ axes show histograms of measurement count at varying fast azimuth and depth. Central plot is coloured by the density of measurements. A linear regression line is fitted to highlight variations with depth. Right column: Delay time versus depth. 


\subsubsection{Comparison of fast azimuths to stress models and fault ori- entations}

We compare our spatially averaged SWS results to other stress models, as well as to fault orientations, using the methods described in Section 4.3.5 (Figure 4.12). In each case, the two angles are compared by taking the absolute value of the cosine of the difference between the two compared angles. We compute the mean cosine of the difference between the angles in order to quantitatively identify areas where the compared angles are in "agreement" (close to parallel) versus areas of "disagreement" (close to perpendicular). The resulting values range from 0 to 1 , where 0 represents perpendicular and 1 represents parallel azimuths between the two angles. The mean and median of the absolute value of the cosine of the difference between the compared angles is calculated and used to identify areas where angles are parallel versus perpendicular, specifically for the entire dataset of compared points, as well as for measurements occurring within Boxes 1-4. Histograms of the frequency of the cosines of the difference of the angles for each area are shown in Figure 4.13.

\section{Fault orientations}

Figures 4.12A and 4.13A compare fast azimuths to the averaged fault orientations. Using the entire dataset we compute an average cosine of the difference of the angles and standard error of $0.83 \pm 0.02\left(34^{\circ}\right.$; median of $\left.0.92,23^{\circ}\right)$, indicating an overall strong correlation between the compared angles (Figure 4.13A). Across the NIDFB (Box 1), the NE-SW striking fault orientations closely match that of our fast azimuths with an average of $0.89 \pm 0.02\left(28^{\circ}\right.$; median of $\left.0.94,1^{\circ}\right)$. However, a departure in fast azimuths from the NE-SW directions to mainly E-W directions occurs in Box 2, demonstrating a strong disagreement between fault orientations and our fast azimuths with an average of $0.69 \pm 0.09\left(46^{\circ}\right.$; median of $\left.0.81,36^{\circ}\right)$. Box 3 also exhibits mainly E-W striking fast azimuths and has an average of $0.5 \pm 0.21$ $\left(60^{\circ}\right.$; median of $\left.0.48,61^{\circ}\right)$, with a general disagreement between the compared angles; however there are significantly fewer faults in Box 3 to compare to our fast azimuths. Box 4 highlights an area of varying fast azimuths and fault directions around Mt. Ruapehu and showed a stronger agreement compared to Boxes 2 and 3 with an average of $0.75 \pm 0.1\left(42^{\circ}\right.$; median of $\left.0.86,31^{\circ}\right)$. 

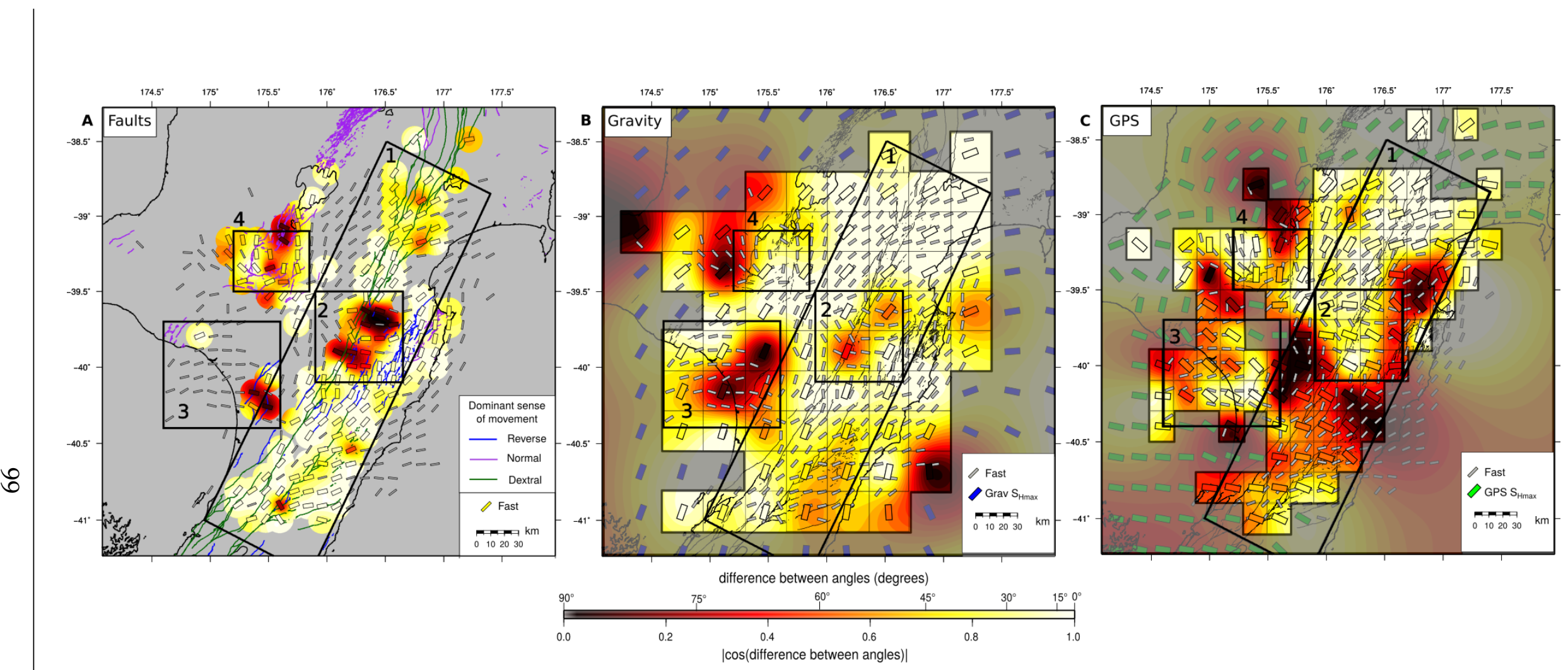

Figure 4.12: A: Averaged fault orientations, from Langridge et al. (2016), are compared to fast azimuths using a 0.1 degree search radius. The two angles are compared by taking the absolute value of the cosine of the difference between the angles. The resulting values range from 0 to 1 , where 0 represents perpendicular and 1 represents parallel azimuths between the two angles. Boxes marked 1-4 highlight areas of interest discussed in the text. B: $S_{H \text { max }}$ from gravity, $S_{H \text { max }}^{\text {grav }}$ are compared to the averaged fast azimuths for each grid-block. C: GPS-derived $S_{H \text { max }}$ directions are compared to averaged fast azimuths for each grid-block. Area is masked to show only grid-blocks for which measurements were compared. 

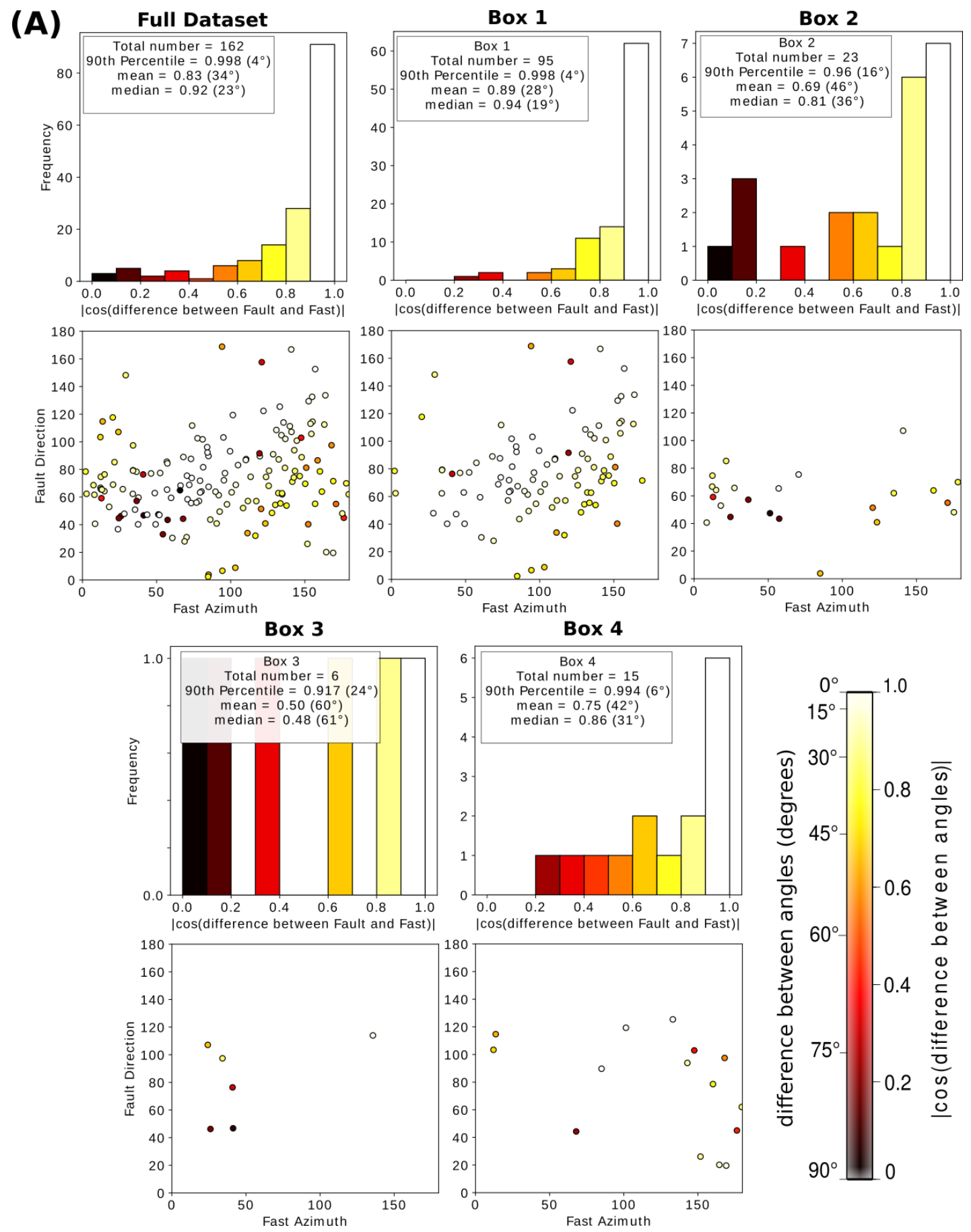

Figure 4.13: Three page figure. Histogram and scatter plots for comparison of fast azimuths to (A) fault orientations, (B) $S_{H \text { max }}^{\text {grav }}$, and (C) GPS-derived $S_{H \max }$. The residual was calculated by taking the cosine of the difference of the compared angles, resulting in a scale from $0-1$, where 0 are perpendicular angles and 1 are parallel angles. Plots show results using both the full dataset of measurements, as well as measurements in Boxes 1-4 from Figure 4.12. We calculate the 90th percentile, mean and median of the cosine of the difference between the angles and provide the total number of compared angles for each area. 

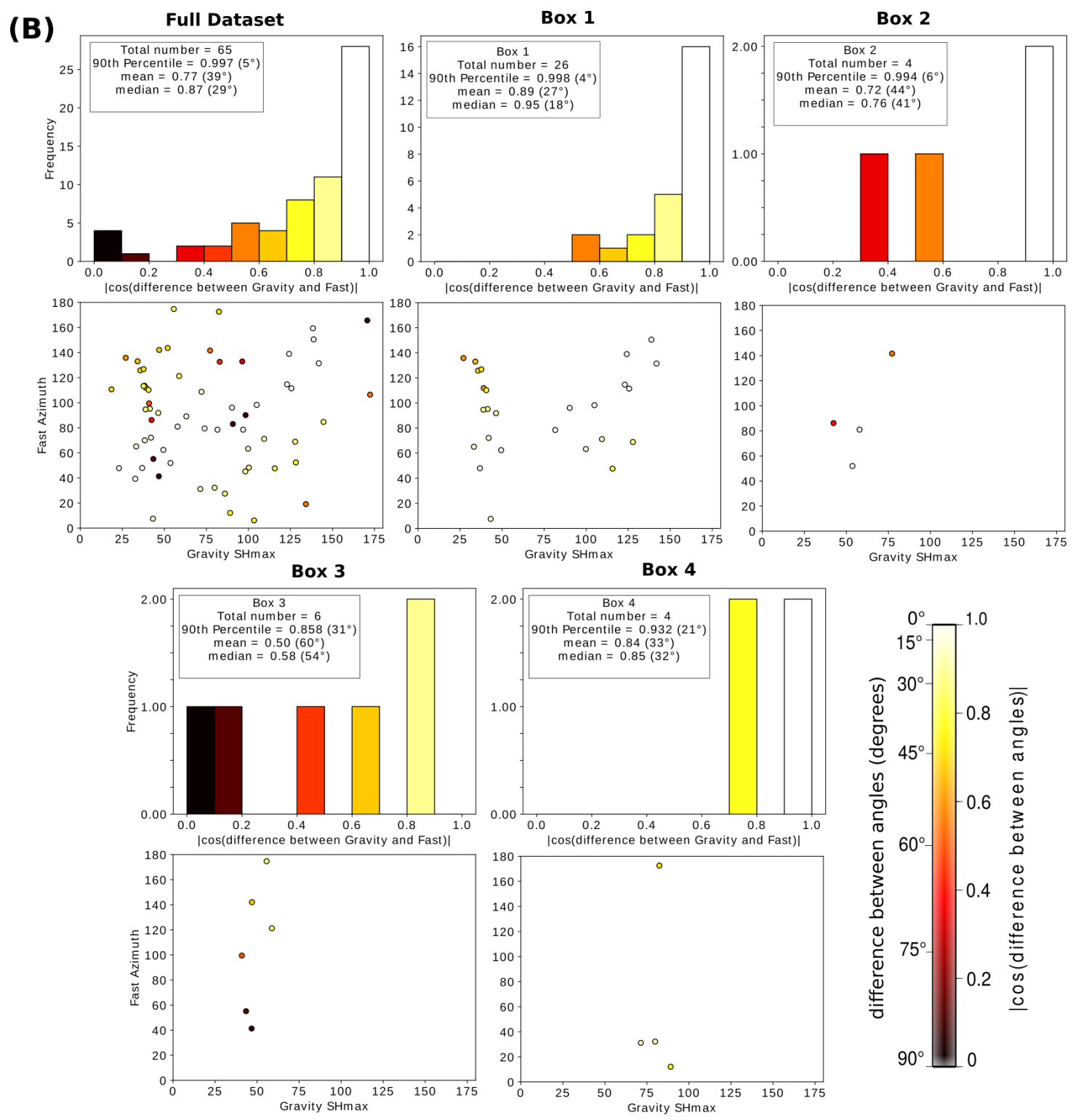

Figure 4.13: (B) Comparison of fast azimuths to $S_{H \max }^{g r a v}$

\section{Gravity}

Figures 4.12B and 4.13B compare $S_{\text {Hmax }}^{\text {grav }}$ to the averaged fast azimuths in each gridblock. The average cosine of the angles and standard error for the SWS azimuth to $S_{H \max }^{\text {grav }}$ comparison is $0.77 \pm 0.03\left(39^{\circ}\right.$; median of $0.87,2^{\circ}$; Figure $\left.4.13 \mathrm{~B}\right)$, indicating that much of the region exhibits fast azimuths which are in close agreement with $S_{\text {Hmax }}^{\text {grav }}$, similar to that of the comparison of fault orientations to our fast azimuths. As with the fault orientations, $S_{H \max }^{\text {grav }}$ is oriented NE-SW along the NIDFB and matches well to our fast azimuths. Box 1 shows a general agreement with an average of $0.89 \pm 0.03\left(27^{\circ}\right.$; median of $\left.0.95,18^{\circ}\right)$. Box 2 has an average of $0.72 \pm 0.36$ 
(C)
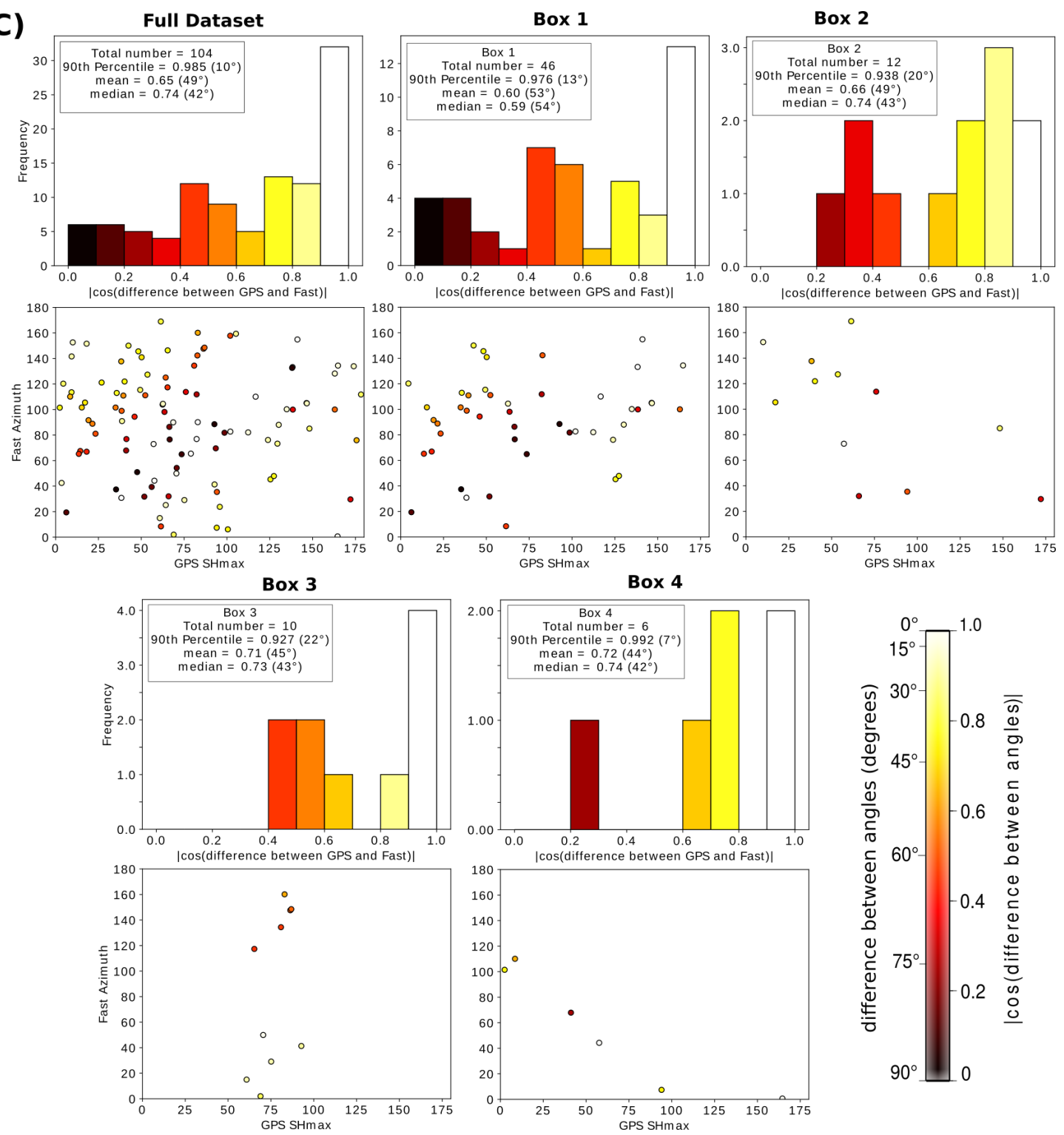

Figure 4.13: (C) Comparison of fast azimuths to GPS-derived $S_{H \max }$.

$\left(44^{\circ}\right.$; median of $\left.0.76,41^{\circ}\right)$. Box 3 shows the weakest agreement with an average of $0.5 \pm 0.21\left(60^{\circ}\right.$; median of $\left.0.58,54^{\circ}\right)$. There is strong agreement between $S_{\text {Hmax }}^{\text {grav }}$ and our SWS measurements in the Mt. Ruapehu region, Box 4, with an average of $0.84 \pm 0.42\left(33^{\circ}\right.$; median of $\left.0.85,32^{\circ}\right)$.

\section{GPS}

Figures $4.12 \mathrm{C}$ and $4.13 \mathrm{C}$ show a comparison of GPS-derived $S_{H \max }$ to the averaged fast azimuths in each grid-block and has an average cosine of the angles of $0.65 \pm 0.03\left(49^{\circ}\right.$; median of $0.74,42^{\circ}$; Figure $\left.4.13 \mathrm{C}\right)$, with the overall weakest agreement between the angles. Across the NIDFB, GPS-derived $S_{H \max }$ directions are 
oriented NW-SE in the southern parts of North Island and NE-SW in the northern parts on North Island. This difference in directions across the NIDFB results in stronger disagreement to our fast azimuths in the south and more agreement in the north, along the NIDFB. Interestingly, the transition between the $S_{H \max }$ directions coincides with the transition in our fast azimuths in Box 2. The average at Box 1 is $0.6 \pm 0.03\left(53^{\circ}\right.$; median of $\left.0.59,54^{\circ}\right)$. Box 2 has an average of $0.66 \pm 0.19\left(49^{\circ}\right.$; median of $\left.0.74,43^{\circ}\right)$. Box 3 has an average of $0.71 \pm 0.22\left(45^{\circ}\right.$; median of $\left.0.73,43^{\circ}\right)$. Finally Box 4 had an average of $0.72 \pm 0.3\left(44^{\circ}\right.$; median of $\left.0.74,42^{\circ}\right)$.

\subsubsection{Spatial averaging of delay times}

Spatially normalizing the delay times of earthquakes located above $30 \mathrm{~km}$ allows us to determine spatial variations in the magnitude of seismic anisotropy (Figure 4.14). We observe significant variations in normalized delay time across North Island. The highest delay times are located in the east, closest to the Hikurangi margin. Larger delay times are also observed around Mt. Ruapehu. Lower delay times are observed across the Wanganui Basin, however station coverage here is limited so we do not interpret results from this area in much detail. The similarity between the spatially averaged delay times to our station averaged delay times suggests that the spatial normalizing of delay times is an appropriate method. Delay times are also compared to the gravity anomaly (McCubbine et al., 2017), and a $30 \mathrm{~km}$ depth averaged $V_{P} / V_{S}$ (Figure 4.14), after Eberhart-Phillips et al. (2010). The gravity anomaly shows increasingly negative values across the Wanganui Basin and towards the northeast, indicative of an increased sediment thickness (up to 5 $\mathrm{km}$ thick; Tozer et al., 2017). $V_{P} / V_{S}$ values are averaged to $30 \mathrm{~km}$ depth and show an increase across North Island, towards the Hikurangi Margin (Eberhart-Phillips et al., 2010).

\subsection{Discussion}

\subsubsection{Fast polarization azimuths}

Fast azimuth averages at individual stations show significant variations across central North Island (Figure 4.9 and Table 4.1). Spatially averaging our fast azimuths allows us to observe variations in fast azimuths in greater detail and helps 

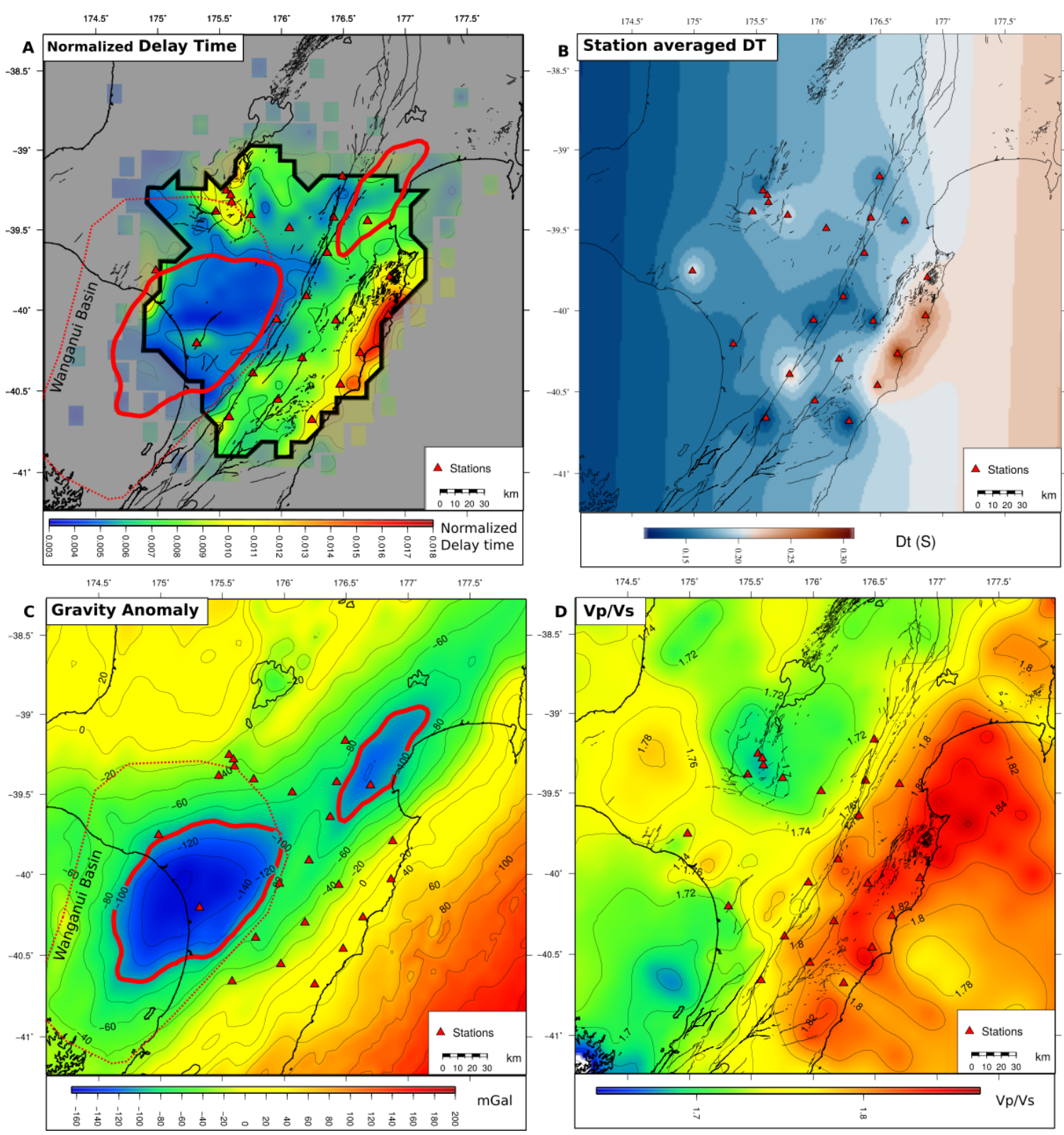

Figure 4.14: A) Spatial averaging of shear wave splitting delay times. Red triangles indicate locations of stations. The area of greatest confidence is marked by the black contour from Figure 4.7. The thick red contours mark the $100 \mathrm{mGal}$ contour from inset C. The extent of Wanganui Basin is marked by the dotted red line (Tozer et al., 2017). B) A surface of the average shear wave splitting delay time (seconds) at each station. C) Gravity anomaly (in mGals; McCubbine et al., 2017). D) 30 $\mathrm{km}$ depth average of $V_{P} / V_{S}$ [after Eberhart-Phillips et al. (2010)].

reveal areas across central North Island where fast azimuths vary from the overall NE-SW trend. We compare our results to a previous extensive study of crustal anisotropy in North Island (Illsley-Kemp et al., 2019). Illsley-Kemp et al. (2019) observe similar spatially averaged fast azimuths with dominant NE-SW fast azimuths along the NIDFB and suggest that their observed NE-SW fast azimuths 
are controlled by a strike-slip stress field with $S_{H \max }$ oriented sub-parallel to the strike of the subduction zone. We examine this in greater detail by comparing our fast azimuths to the average orientation of nearby faults, GPS-derived $S_{H} \max$ and gravity-induced stress, $S_{\text {Hmax }}^{\text {grav }}$ (Figure 4.12).

Across the central North Island, using the entire dataset, the fault orientations presented the strongest agreement with our fast azimuths with an average and standard error of $0.83 \pm 0.02\left(34^{\circ}\right.$; median of $\left.0.92,23^{\circ}\right)$, suggesting that the structural fabric of the faults play a key role in our observed crustal anisotropy directions (Figures $4.12 \mathrm{~A}$ and $4.13 \mathrm{~A}$ ). $S_{H \max }^{\text {grav }}$ directions are close to parallel to the NE-SW trending Hikurangi Margin and have the second strongest agreement to our fast azimuths with an average of $0.77 \pm 0.03\left(39^{\circ}\right.$; median of $\left.0.87,29^{\circ}\right)$, suggesting that topographically induced stresses may also play a role in our observed stress-field from crustal anisotropy (Figures 4.12B and 4.13B). GPS-derived $S_{H} \max$ directions across North Island change from trench-perpendicular directions in the south to trench-parallel directions in the north. In southern North Island, GPSderived $S_{H} \max$ are oriented mainly E-W, likely due to elastic strain accumulation as a result of locking on the underlying plate in this region (Hirschberg et al., 2017). The E-W GPS-derived $S_{H} \max$ in southern North Island are oriented perpendicular to our NE-SW azimuths. GPS-derived $S_{H} \max$ were least similar to our fast azimuths with an average of $0.65 \pm 0.03\left(49^{\circ}\right.$; median of $0.74,42^{\circ}$; Figure $4.12 \mathrm{C}$ and 4.13C). Townend et al. (2012) use earthquake focal mechanisms to invert for the stress regime in New Zealand. The tectonic stresses observed by Townend et al. (2012) along the NIDFB (Figure 4.15) show $S_{\text {Hmax }}$ directions oriented NE-SW (parallel to the NIDFB) in northeastern and southern North Island, with slightly more variations across the central part of our study region, as well as in the Mt. Ruapehu region. Because the $S_{H \max }$ measurements from focal mechanisms were limited we do not interpret the comparison with our SWS results in great detail (Figure 4.15).

\section{NIDFB - Box 1}

Box 1 encompasses the NIDFB, where our spatially averaged fast azimuths are mainly NE-SW, parallel and sub-parallel to the strike of the NIDFB (Box 1; Figure 4.10). Previous SWS studies observe NE-SW trending $\left(30^{\circ}-60^{\circ}\right)$ fast azimuths along the NIDFB (e.g., Gledhill and Stuart, 1996; Audoine et al., 2000, 2004; Morley et al., 2006; Illsley-Kemp et al., 2019). Patterns of NE-SW trending anisotropy have 


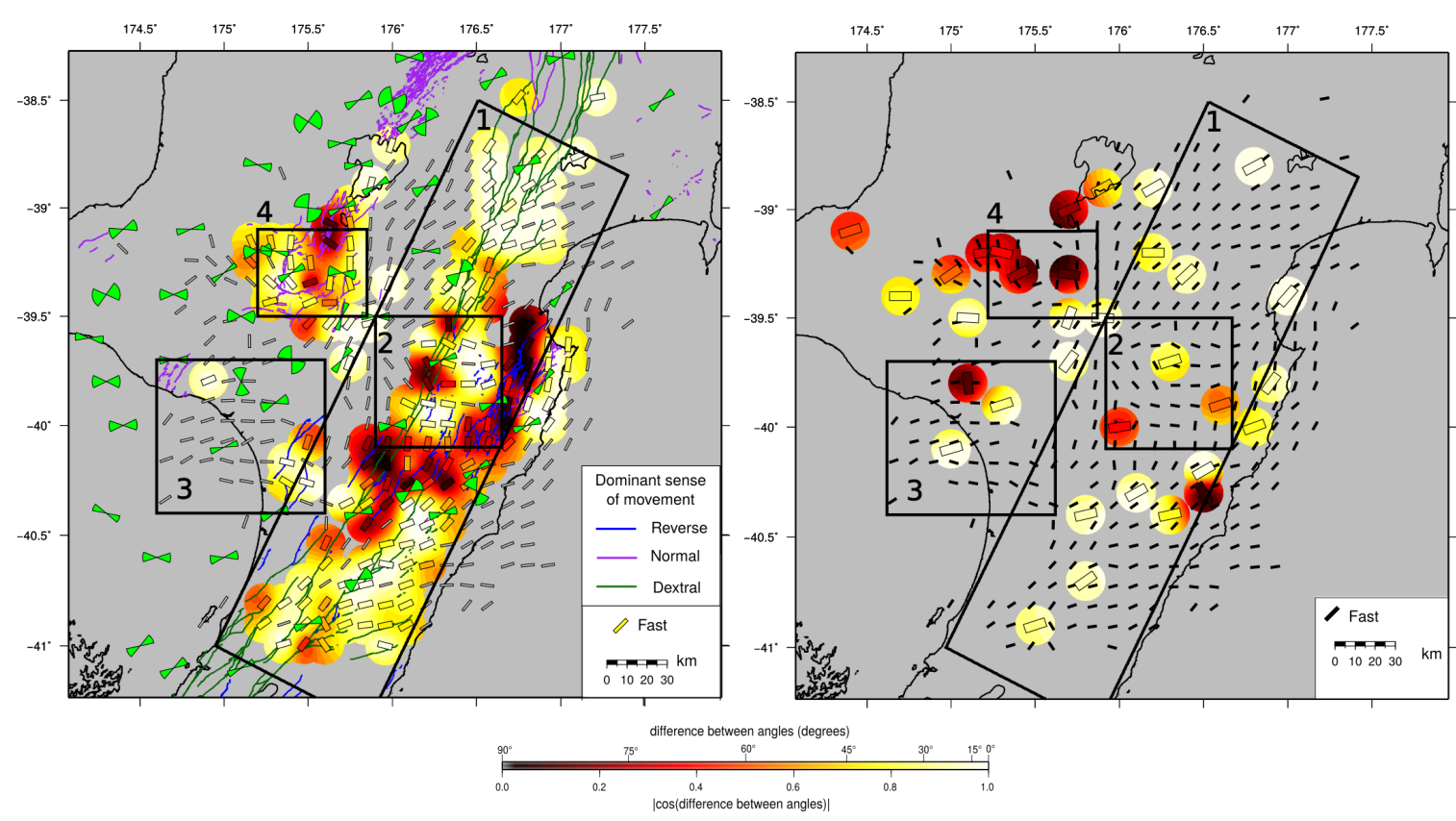

Figure 4.15: Left: Averaged fault-derived $S_{H \max }$ orientations, from Langridge et al. (2016), are compared to fast azimuths using a 0.1 degree search radius. Fault-derived $S_{H \max }$ by adding $90^{\circ}$ to all reverse faults and $45^{\circ}$ to all dextral faults, based on the Andersonian model for stress on faults (Figure 4.4; Anderson, 1905). Green bow-ties are $S_{H} \max$ directions derived from focal mechanisms (Townend et al., 2012). Right: $S_{H} \max$ directions derived from focal mechanisms (Townend et al., 2012) are compared to fast azimuths using a 0.1 degree search radius.

been observed in P-wave anisotropy and are especially prevalent along the NIDFB (Eberhart-Phillips and Reyners, 2009). Just south of our study area, in the Wellington region, Evanzia et al. (2017) observe NE-SW trending anisotropy, except in the Wanganui Basin where anisotropy is trench perpendicular.

The comparison analyses within Box 1 of our fast azimuths to fault orientations, $S_{H \max }^{\text {grav }}$ and GPS-derived $S_{H \max }$ results in an average cosine of the difference of the angles of $0.89 \pm 0.02\left(28^{\circ}\right.$; median of $\left.0.94,19^{\circ}\right), 0.89 \pm 0.03\left(27^{\circ}\right.$; median of 0.95 , $\left.18^{\circ}\right)$, and $0.6 \pm 0.03\left(53^{\circ}\right.$; median of $\left.0.59,54^{\circ}\right)$, respectively (Figures 4.12 and 4.13 ). The strong agreement between fast azimuths and the fault orientations, as well as

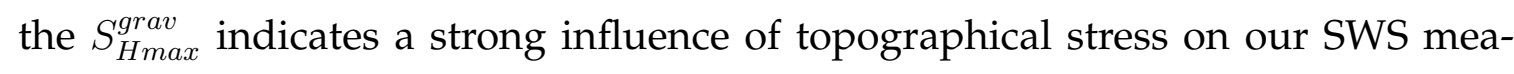
surements along the NIDFB (Figures 4.12A and B). Fast azimuths that are oriented NE-SW along the NIDFB are interpreted as being controlled by the faulting fabric of the NIDFB. 


\section{Central NIDFB - Boxes 2 and 3}

An exception to the NE-SW trend along the NIDFB is highlighted by Box 2 (Figure 4.10). Fast azimuths in Box 2 exhibited predominantly NW-SE and E-W directions, perpendicular to those observed across the rest of Box 1 . The comparison analyses within Box 2 of our fast azimuths to fault orientations, $S_{H \max }^{\text {grav }}$, and GPS-derived $S_{H \max }$ results in an average cosine of the difference of the angles of $0.69 \pm 0.09$ $\left(46^{\circ}\right.$; median of $\left.0.81,36^{\circ}\right), 0.72 \pm 0.36\left(44^{\circ}\right.$; median of $\left.0.76,41^{\circ}\right)$, and $0.66 \pm 0.19\left(49^{\circ}\right.$; medium of $0.74,43^{\circ}$ ), respectively. The observed change in GPS-derived $S_{H \max }$ from NE-SW north of Box 2 to NW-SE south of Box 2 coincides with the location of our E-W trending fast azimuths (Figure 4.12C). The locations of Boxes 2 and 3 both also coincide with areas of negative gravity anomaly (around $-160 \mathrm{mGal}$; Figure 4.14C; McCubbine et al., 2017). Box 3 is located within the Wanganui sedimentary basin and the contours of the negative Bouguer gravity anomaly coincide with and approximately mimic those of the sedimentary isopachs (Harmsen, 1985). The area in Box 3 is characterized by $\sim 5 \mathrm{~km}$ thick Pliocene-Pleistocene shoreline shelf marine sediment (Carter and Naish, 1998; Harmsen, 1985). The large negative gravity anomaly observed in the Wanganui Basin extends to the northeast, towards the East Coast basin and across Box 2 (Figure 4.14C). These basins do not appear to be directly related to faulting and instead form as a crustal down-warp or pulldown structure, which accumulates thick sediment (Stern et al., 1992). Several mechanisms have been suggested for the forces that create these type of basins, including plate interface frictional stresses (Stern et al., 1992), mantle flow forces (Kudo and Yamaoka, 2003), and lithospheric thickening (Stern et al., 2006), and compressional stresses (Sierd Cloetingh and Groenewoud, 1989).

While NE-SW trending P-wave anisotropy has been observed by Eberhart-Phillips and Reyners (2009) across most of the NIDFB, their results showed a sharp deviation to a NW-SE trend coinciding with our observed E-W directions within Box 2. They observe that the rapid change from NE-SW to NW-SE occurring south of the TVZ also coincides with a change in the GPS derived azimuths of $S_{\text {Hmax }}$, modeled by Beavan et al. (2007), from approximately NE, east of Lake Taupo, to approximately NW, south of the TVZ, and suggest a possible effect of stressinduced anisotropy in the brittle part of the crust in this region (Eberhart-Phillips and Reyners, 2009). 
The transition to dominantly E-W fast azimuths in Box 2 is located $\sim 20 \mathrm{~km}$ north of the observed change in geodetically and seismologically inferred coupling (Figure 4.10; Wallace et al., 2004). Townend et al. (2012) have suggested that the transition in their $S_{H \max }$ orientations from NE-SE to ENE-WSW also roughly coincides with the coupling transition line (Figure 4.10).

An area of reverse faulting, located on the eastern side of the NIDFB, south-west of Napier, also roughly coincides with the area of E-W azimuths in Box 2 (Figure 4.10 ), however most of the previously mapped faults are located east of Box 2 . In a reverse faulting regime $S_{H \max }$ will be oriented perpendicular to the strike of the fault (Figure $4.13 \mathrm{~A}$ ). However, by comparing the fault-derived $S_{H \max }$ to our fast azimuths (Figure 4.15), we find significant disagreement between the two angles in areas of reverse faulting, east of the NIDFB, suggesting that our fast azimuths are not controlled by the reverse faulting east of Box 2. Further, we compare our fast azimuths to $S_{H} \max$ directions derived from focal mechanisms (Townend et al., 2012) and observe areas in Box 2 with both agreement and disagreement (Figure 4.15). Additionally, the sparsity of $S_{H} \max$ directions make interpretation difficult.

We suggest that the E-W directions in Box 2 are likely stress-induced and related to the transition of GPS-derived $S_{H \max }$ from NE-SW to NW-SE in this region (Figure $4.12 \mathrm{C}$ ). We suggest that the thick sediment layer in this region may contribute to weaken the strong NE-SW oriented fault fabric observed in our fast azimuths across most of the NIDFB, allowing us to observe stresses occurring on the plate interface instead of structurally-controlled anisotropy.

Box 3 highlights an area across the Wanganui Basin where fast azimuths are oriented mainly E-W, similar to Box 2. However, station coverage in this area is sparse making interpretation difficult. The comparison analyses within Box 3 of our fast azimuths to fault orientations, $S_{\text {Hmax }}^{\text {grav }}$, and GPS-derived $S_{H \max }$ results in an average cosine of the difference of the angles of $0.5 \pm 0.21\left(60^{\circ}\right.$; median of $\left.0.48,61^{\circ}\right)$, $0.5 \pm 0.21\left(60^{\circ}\right.$; median of $\left.0.58,54^{\circ}\right)$, and $0.71 \pm 0.22\left(45^{\circ}\right.$; median of $\left.0.73,43^{\circ}\right)$, respectively. Here, the GPS-derived $S_{H \max }$ has the strongest agreement to our fast azimuths, suggesting that this area may be controlled by stresses on the plate interface where $S_{H} \max$ directions are oriented sub-parallel to the E-W direction of apparent plate motion (Altamimi et al., 2012). This region also coincides with the largest negative Bouguer anomaly, partly a result of the $\sim 5 \mathrm{~km}$ thick sediment 
layer. Similar to our interpretation in Box 2, we suggest that the thick sediment in this region may contribute to the observation of stress-induced anisotropy rather than of structural anisotropy.

\section{Mt. Ruapehu - Box 4}

In the southern TVZ, around Mt. Ruapehu, fast directions vary significantly between stations with E-W trending directions, at stations MTVZ and WHVZ located on the southwestern and northeastern flanks, respectively, and stations FWVZ and WNVZ with N-S directions, located on the northwestern and southeastern flanks, respectively (Figure 4.9). The spatial averaging of our fast azimuths results in fast azimuths around Mt. Ruapehu with a prominent tangential trend around the volcano (Box 4; Figure 4.10). The comparison analyses within Box 4 of our fast azimuths to fault orientations, $S_{H \max }^{\text {grav }}$, and GPS-derived $S_{H \max }$ results in an average cosine of the difference of the angles of $0.75 \pm 0.1\left(42^{\circ}\right.$; median of $\left.0.86,31^{\circ}\right)$, $0.84 \pm 0.42\left(33^{\circ}\right.$; median of $\left.0.85,32^{\circ}\right)$, and $0.72 \pm 0.3\left(44^{\circ}\right.$; median of $\left.0.74,42^{\circ}\right)$, respectively. We observe relatively similar agreement between all three compared directions and our fast azimuths, suggesting a complex contribution from both stress-induced and structural anisotropy.

The variation between N-S and E-W fast azimuths at Mt. Ruapehu has been previously observed by Johnson et al. (2011). They show that the anisotropy can be divided into regions where the fast azimuths match stress estimations from focal mechanism inversions, indicating stress-induced anisotropy, and areas where fast azimuths align with structural features such as faults, indicating structural anisotropy.

Faults in the TVZ are predominantly normal faults (due to the extensional regime), striking NE-SW (Beanland and Haines, 1998). However, the active faults around Mt. Ruapehu, created by subsidence in the central axis of the TVZ, are downthrown towards the mountain, creating a caldera subsidence on a 'ring' of faults around Mt. Ruapehu (Villamor and Berryman, 2006). The complex N-S and E-W normal fault patterns around Mt.Ruapehu closely mimics our observed variations in fast azimuths, suggesting some structural control on crustal anisotropy in this region.

Earlier studies of seismic anisotropy at Mt.Ruapehu using SWS observe tempo- 
ral changes in the orientation of crustal anisotropy, interpreted as being caused by reoriented cracks due to repeated filling or depressurizing of magmatic dike systems after the 1995-1996 eruptions (Miller and Savage, 2001; Gerst and Savage, 2004). Illsley-Kemp et al. (2019) also examined crustal SWS results north of Mt. Ruapehu, across the TVZ, and find dominant rift-aligned fast azimuths in the central TVZ, suggesting a strong control by rift-related extensional stresses, similar to directions observed by Audoine et al. (2004). However, extension rates in the southern TVZ are significantly smaller (Beavan et al., 2016) creating a smaller control on fast azimuths in this region. It is difficult to distinguish between structural and stress-induced anisotropy in such an environment, however, our measurements suggest that both stress and structure influences our fast azimuths around Mt. Ruapehu.

\subsubsection{Delay times}

Spatially normalizing the delay times from earthquakes above $30 \mathrm{~km}$ allows us to examine spatial variations in the magnitude of seismic anisotropy (Figure 4.14A). Normalized delay times are generally higher further east and closer to the Hikurangi Margin and lower further west, inland and across the Wanganui Basin. We compare our spatially normalized delay times to station averaged delay times (Figure 4.14B). The similarity between the spatially averaged delay times to our station averaged delay times (Figure 4.14B) suggests that the spatial normalization of delay times is an appropriate method. The median delay times at each station varies from $0.115-0.245 \mathrm{~s}$ and are comparable to results in other subduction zones (e.g., Fouch and Fischer, 1998; Nakajima and Hasegawa, 2004).

Similarly high delay times have been previously observed east of the NIDFB in central North Island and are consistent with increasing horizontal stresses and increasing crack density towards the Hikurangi trench (Illsley-Kemp et al., 2019). Illsley-Kemp et al. (2019) also observe higher delay times north of Hawkes Bay, which also increase more gradually towards the Hikurangi trench. They attribute this trend to higher fluid content and pervasive fractures towards the Hikurangi trench.

Spatially averaged delay times are also compared to the gravity anomaly (Figure 4.14C), after McCubbine et al. (2017), and a $30 \mathrm{~km}$ depth averaged $V_{P} / V_{S}$ (Fig- 
ure 4.14D), after Eberhart-Phillips et al. (2010). The offshore east North Island region coincides with a high $V_{P} / V_{S}$ area $(\sim 1.8)$, and is interpreted as a fluid-rich and highly over-pressurized region (Eberhart-Phillips et al., 2010). The gravity anomaly shows increasingly negative values across the Wanganui Basin and towards the northeast, indicative of an increased sediment thickness (up to $\sim 5 \mathrm{~km}$; Tozer et al., 2017).

We observe a distinct transition between low and high delay times south of the TVZ and across the NIDFB. Generally, west of the NIDFB, across the Wanganui Basin, delay times are significantly lower and the area of low delay times coincides with an area of thick sediment (up to $4 \mathrm{~km}$ thick; Harmsen, 1985). This suggests that fluid-filled cracks in the top few kilometers of the Wanganui Basin are more randomly oriented, compared to cracks in the east, which are located close to the shallow subduction zone. The Wanganui region also coincides with an area of high $V_{P} / V_{S}$ values ( 1.76; averaged over the top $\left.30 \mathrm{~km}\right)$, consistent with area of increased fluids (Eberhart-Phillips et al., 2010). We note that the station coverage across the Wanganui Basin was limited and we do not express as high a confidence in the delay time results across this region as we do for results along the east coast. The increasing delay time trend we observe towards the Hikurangi margin correlates well with increasing $V_{P} / V_{S}$ measurements, and suggests increasing horizontal stresses and/or crack density towards the Hikurangi trench and within the top few kilometers of the crust.

\subsection{Conclusions}

We have analysed crustal anisotropy across central North Island using over 20,000 local earthquakes recorded on 24 GeoNet stations. The density of our measurements allowed us to investigate, in high resolution, the causes of spatial variations in anisotropy throughout central North Island.

We have found that:

1. Spatially averaged fast azimuths show a dominant NE-SW orientation along the NIDFB. Fault orientations show the closest agreement to our fast azimuths, suggesting a pervasive structural control. A strong agreement with $S_{\text {Hmax }}^{\text {grav }}$ along the NIDFB provides further support for this structurally con- 
trolled anisotropy.

2. Fast azimuths in the Wanganui Basin exhibit a strong E-W trend and are most similar to the regional GPS-derived $S_{H \max }$. We suggest that the thick sediments in this region act to weaken the crustal fabric and our fast azimuths may more closely represent the stress field at greater depths where $S_{H} \max$ directions are more closely aligned with the apparent plate motion.

3. A clear change in fast azimuths from NE-SW to NW-SE and E-W occurs within a small area along the NIDFB, southeast of the TVZ, and coincides with the transition from an extensional regime in the north to one that is compressional in the southern North Island, consistent with a dominantly E-W GPS-derived $S_{H} \max$ in the south. Similar to the Wanganui Basin, this region also coincides with thick sediments from the East Coast Basin, and we suggest that the fast azimuths may be reflective of the stress field rather than structurally controlled.

4. Fast azimuths around Mt. Ruapehu show a general agreement with both GPS-derived $S_{H} \max$ as well as local fault orientations. Previous studies have suggested both stress-induced and structural controls in the vicinity of the volcano and our results support these suggestions.

5. Spatially averaging the delay times reveals a significant increase in delay time values towards the Hikurangi trench, similar to previous results, and suggests high-fluid content and pervasive fractures towards the Hikurangi trench and within the top few kilometers of the crust.

6. We searched for temporal variations in SWS delay times, fast azimuths and $V_{P} / V_{S}$ measurements during the 2010 Manawatu SSE; however, our results do not show any significant changes during the life of this SSE. This suggests that variations are too small to be observed with our dataset, making it a nonideal event for detecting temporal SWS variations. 


\section{Chapter 5}

\section{Upper mantle anisotropy across the Taranaki-Ruapehu Line in North Island, New Zealand}

\subsection{Abstract}

We investigate the relationship between seismic anisotropy and mantle deformation across a deep boundary in the crust and mantle, marked by the TaranakiRuapehu Line (TRL), in the back-arc region of western North Island, New Zealand. We used shear wave splitting (SWS) to study seismic anisotropy measurements across the TRL between Taranaki and Ruapehu volcanoes; where deeper crust ( $\sim 32 \mathrm{~km}$ thick) to the south and a shallower crust ( $\sim 25 \mathrm{~km}$ thick) in the north is interpreted as an abrupt step in the Moho. Numerous geophysical studies have examined changes across the TRL, such as in electrical conductivity, gravity, seismic attenuation, and crustal thickness, but few studies have provided constraints on the upper mantle structure from seismic anisotropy. Addressing this gap in knowledge, we studied upper mantle anisotropic properties across the TRL by analyzing teleseismic XKS events recorded on 10 temporary broadband seismic stations on the Ruapehu And Taranaki Teleseismic Imaging Line (RATTIL) network between 2012-2014.

Our SWS measurements from teleseismic earthquakes reveal a strong NE-SW oriented anisotropy at each station in the deployment. The average fast azimuth 
is NE-SW $\left(42^{\circ} \pm 5^{\circ}\right)$, subparallel to the trench. The dominant trench-parallel orientation of our fast azimuth measurements are likely due to the NE-SW latticepreferred orientation of olivine in the mantle wedge due to shear deformation associated with oblique convergence and trench-parallel mantle flow. The similarity of our fast azimuths to previous studies in eastern North Island, where there is no wedge under the stations, suggests that similar fast azimuths are found in both the mantle wedge and subslab mantle.

Previous studies have observed apparent isotropy, dominantly west of the central volcanic region, and have suggested that isotropy may extend as far south as the TRL. Our results show that the RATTIL region is anisotropic and we suggest that the boundary between apparent isotropy to the north and anisotropy in the south is located north of the RATTIL network $\left(-38.75^{\circ}\right)$. Minor variations in delay times are observed across the TRL with higher delay times observed at stations closest to the TRL. This suggests some influence from the boundary, however we are unable to constrain the depth of the variations from teleseismic earthquakes alone.

\subsection{Introduction}

In this chapter, we aim to investigate the relationship between seismic anisotropy and strain in the upper mantle across the Taranaki-Ruapehu Line (TRL) using shear wave splitting (SWS) measurements from teleseismic earthquakes. We analyzed SWS on 726 teleseismic earthquakes recorded on 10 temporary seismic stations from the Ruapehu And Taranaki Teleseismic Imaging Line (RATTIL) deployment (between November 2012 and February 2014). We aimed to detect any potential spatial variations in SWS measurements across the TRL in order to better understand strain variations across this boundary. Our results provide new constraints on the upper mantle kinematics across the TRL, where previous studies have reported changes in electrical conductivity (Salmon et al., 2011), seismic attenuation (Salmon et al., 2011), gravity (Stern et al., 1987) and crustal thickness (e.g., Stern et al., 2013; Dimech et al., 2017).

\subsubsection{Tectonic Setting}

The back-arc region of North Island, New Zealand is the site of a unique geophysical boundary located between Taranaki and Ruapehu volcanoes, known as the 


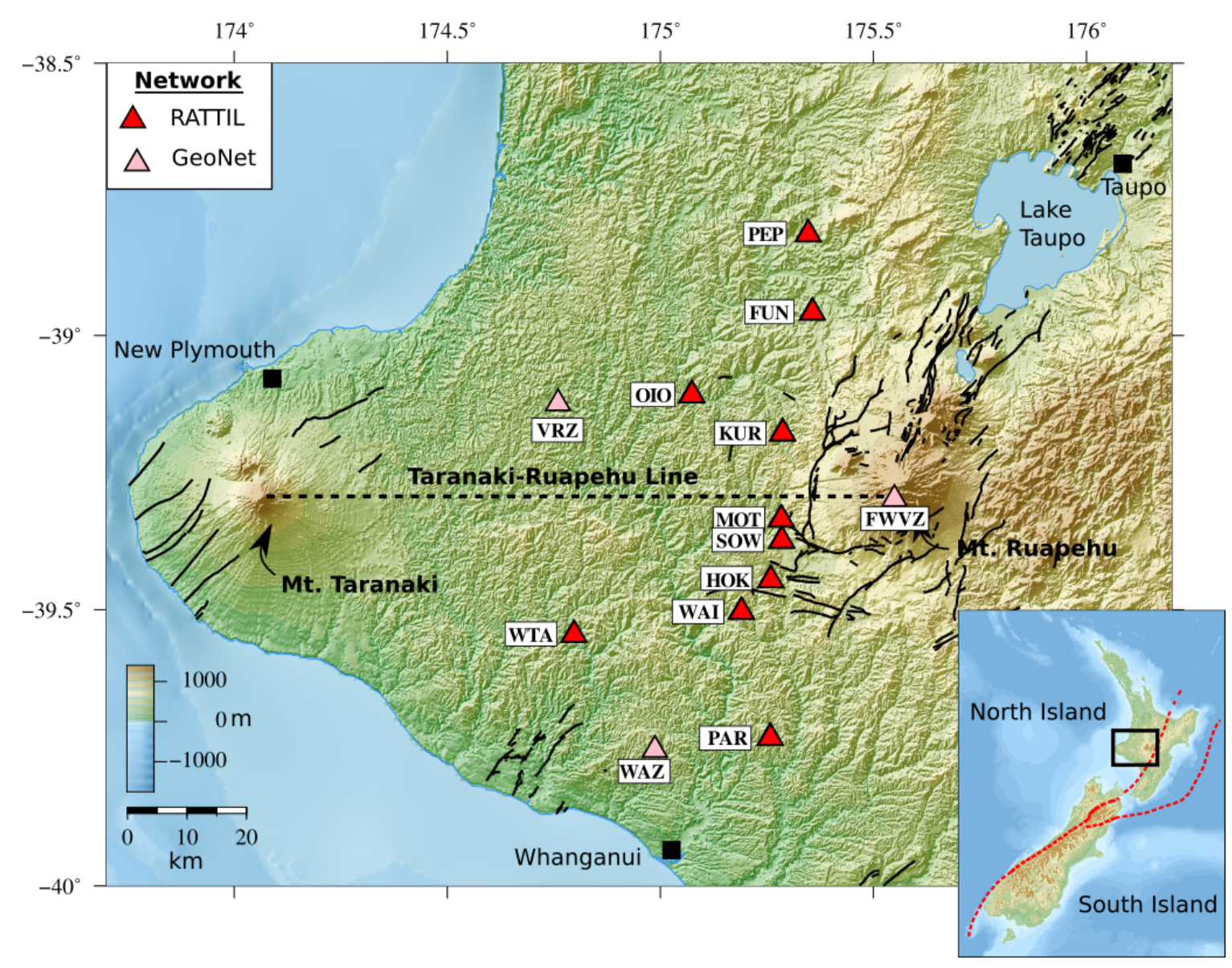

Figure 5.1: Locations of broadband stations in western North Island, New Zealand used in this study from the 2013 Ruapehu And Taranaki Teleseismic Imaging Line (RATTIL) survey in red (Dimech, 2016) and GeoNet stations in pink. The Taranaki-Ruapehu Line is marked by the dashed black line. Active fault traces are from Langridge et al. (2016).

Taranaki-Ruapehu Line (TRL; Figure 5.1). The east-west trending TRL was first recognized more than 40 years ago as a deep boundary in the mantle at which seismic attenuation (Q-1) changes abruptly (Hatherton, 1970; Mooney, 1970). At the western end of the TRL lies Taranaki, an andesitic volcano, and on the eastern end is Mt. Ruapehu, marking the transition point where the horizontal stress regime changes from extensional north of the TRL to predominantly compressional to the south (e.g., Rowland and Sibson, 2001). Previous studies of electrical resistivity (Salmon et al., 2011), seismic attenuation of P waves (Salmon et al., 2011), gravity (Stern et al., 1987), receiver functions (Eberhart-Phillips et al., 2008; Dimech et al., 2017), and seismic anisotropy (Audoine et al., 2004; Greve et al., 2008) have explored variations in geophysical properties across the TRL. A variety of geophysical methods have suggested the presence of a 7-10 km crustal step across the TRL (Figure 5.2; Stern et al., 1987; Seward et al., 2009; Salmon et al., 2011; Tozer 
et al., 2017; Dimech et al., 2017). Based on a sharp gradient in the isostatic gravity anomaly, Stern et al. (1987) estimated a change in crustal thickness on the order of $10 \mathrm{~km}$. An estimated crustal thickness of $\sim 32 \mathrm{~km}$ (Salmon et al., 2011) south of the TRL thins to just $25 \mathrm{~km}$ (Stratford and Stern, 2006; Salmon et al., 2011) north of the line (Figure 5.2). It has been suggested that this step in the crust and Moho is a result of delamination of the lower crust and mantle lithosphere to the north (Stern et al., 2013; Dimech et al., 2017). A magnetotelluric study observed low resistivities (<100 Ohm-m) north of the TRL, at depths greater than $15 \mathrm{~km}$ and significantly higher values (1000 Ohm-m) at depths greater than $15 \mathrm{~km}$ south of the TRL, suggesting a much colder upper mantle south of the TRL (Salmon et al., 2011). A strong change from anisotropy to apparent isotropy was observed in the

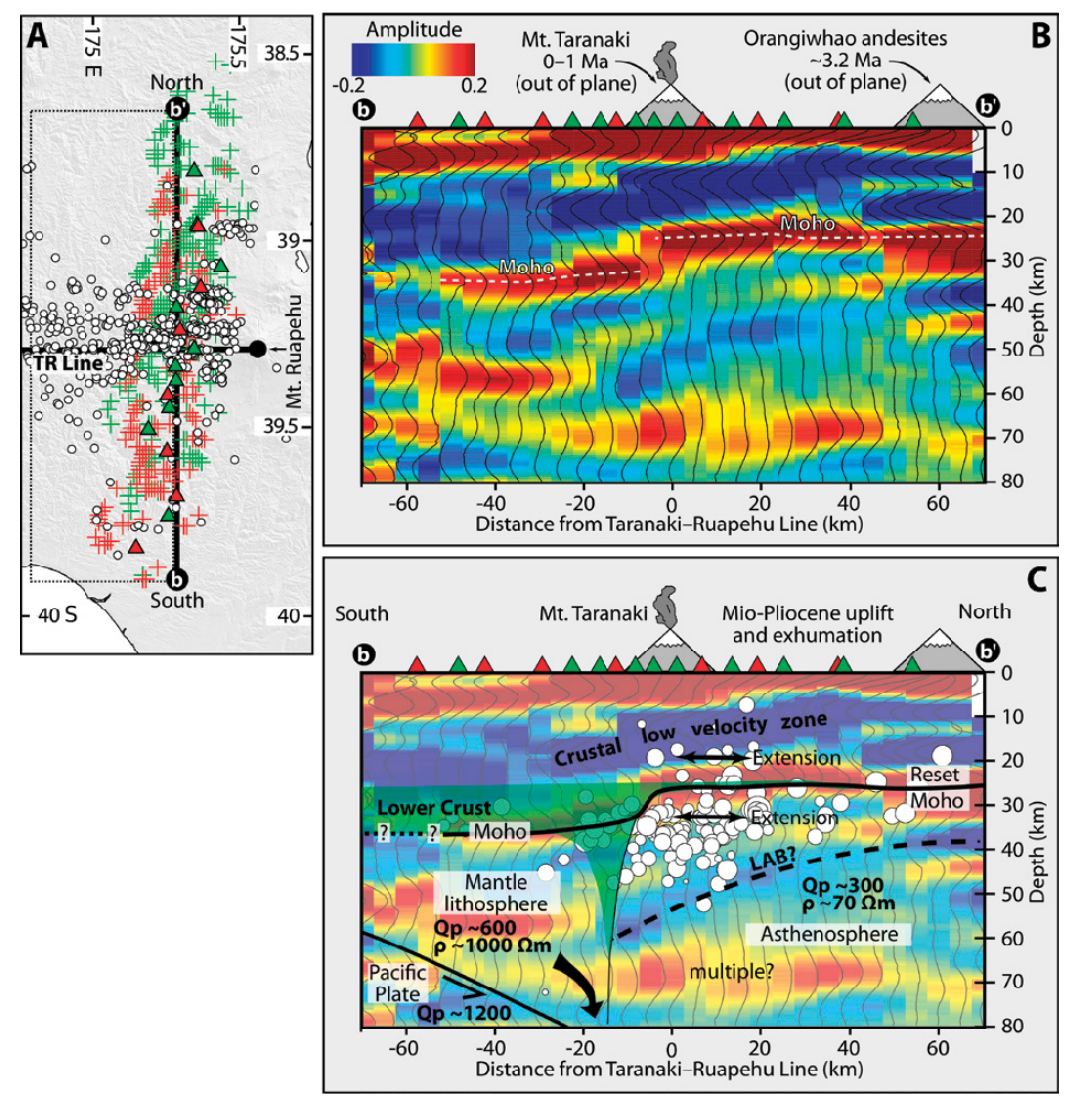

Figure 5.2: Crustal structure profile across the Taranaki Ruapehu Line (TRL) [from Dimech et al. (2017)]. (A) Locations of piercing points for earthquakes used to create common conversion point (CCP) stack shown in B and C. Red triangles mark locations of RATTIL stations. Green triangles mark the location of a previous deployment called the Taranaki Ruapehu Attenuation Profile (TRAP), which operated from 2002-2003. (B) Uninterpreted and (C) interpreted receiver function profile along profile $b-b^{\prime}$. Local earthquakes are shown as white circles and are located in an area of extension, represented by black lines. The green shaded region represents a lower crust that is proposed to be extended and then delaminated (Dimech et al., 2017). 
Waikato region and was suggested to extend as far down as the TRL, but there was not enough data in these studies to constrain the 2-D character of this change (Audoine et al., 2004; Greve et al., 2008).

\subsubsection{Shear Wave Splitting}

Shear wave splitting using teleseismic earthquakes allows one to determine the magnitude and direction of seismic anisotropy in the mantle and is a widely used technique to understand processes affecting the upper mantle. As a shear wave travels through an anisotropic medium, it is split into two orthogonally polarized shear waves travelling at different velocities. The magnitude and orientation of anisotropy is determined by two splitting parameters, $\phi$ the polarization of the fast shear wave (also know as fast azimuth) and $\delta t$, the delay time between the fast and slow waves, which in turn is directly dependent on the thickness of the anisotropic material, as well as the average percent anisotropy. If anisotropy changes along the path, the splitting will be most sensitive to the last layer traversed (e.g., Rumpker and Silver, 1998). Seismic waves from teleseismic earthquakes at radial distances $>80^{\circ}$ travel through the core, where any record of down-going ray anisotropy is reset. The core-refracted phases, including SKS and SKKS (called XKS phases), are commonly used in SWS to avoid source side contamination of the signal. They provide good lateral resolution due to the steep angle of incidence of the phase arrivals (Savage, 1999). The XKS phases of a shear wave are radially polarized from the core-mantle boundary $(\mathrm{CMB})$ to the receiving station and if these radially polarized shear waves encounter an anisotropic layer in the upper mantle or lithosphere on the receiver side, they will split into two orthogonally polarized quasi-shear waves with fast and slow orientations. Thus, shear wave splitting of the upper mantle provides us with important information about the orientation and thickness of anisotropic material between the core-mantle boundary and the station. In comparison to upper mantle anisotropy, with delay times on the order of 1-3 s (e.g., Audoine et al., 2004), the contribution from crustal anisotropy is relatively small, with maximum delay times on the order of 0.2-0.3 s (e.g., Zal et al., 2020; Illsley-Kemp et al., 2019). 


\subsubsection{Upper mantle anisotropy}

Generally, anisotropic domains are randomly oriented. However, when there is a dominant alignment of an anisotropic material, it can produce a measurable anisotropic signal (Savage, 1999). This phenomenon is commonly observed in the upper mantle, where highly anisotropic crystals such as olivine make up $70 \%$ of the Earth's upper mantle and lithosphere (Jeanloz et al., 1989). Within the upper mantle, strain, which may or may not be related directly to the current stress, creates a preferred orientation of mineral crystals, a property called lattice preferred orientation (LPO; Kendall, 2013). By measuring SWS fast azimuths the direction of maximum strain can be inferred (Savage, 1999).

The strain causing the LPO of olivine crystals in the mantle is most classically interpreted as being induced by forces due to lithospheric plate movement over the asthenosphere (e.g., Savage and Silver, 1993; Park and Levin, 2002; Long and Silver, 2009). It may be caused by past or present shear or extension in the lithosphere, or by present flow in the asthenosphere. Although the LPO of olivine is generally considered as the major mechanism for anisotropy in the upper mantle, other factors, such as oriented melt pockets (OMP) and periodic thin layering (PTL) can also contribute to shear wave measurements.

In certain conditions, of pressure and temperature, the $a, b$, and $c$ axes of olivine are known to align with the extension, compression, and intermediate axes of the strain ellipse, respectively (Figure 5.3; Jung and Karato, 2001b). In type-A and type- $C$ fabric of olivine the polarization of the faster $S$ wave will be subparallel to the flow direction (Karato et al., 2008). Type-A olivine typically occurs at low differential stress and water-poor conditions and type-C fabric is observed in water-rich conditions and under low stress (Jung and Karato, 2001b). In contrast, in type-B fabric the fast azimuth will be perpendicular to the shear direction and forms under high stress (>300 MPa) and high water content (Jung and Karato, 2001a), suggesting that B-type fabric of olivine can explain the presence of trenchparallel anisotropy in a region where shear direction is trench-perpendicular (e.g., Audoine et al., 2004).

Additionally, new types of olivine fabric (type-D and E) have also been reported (Katayama et al., 2004). Similar to type-A and C fabrics, in type-D and E fabrics the 


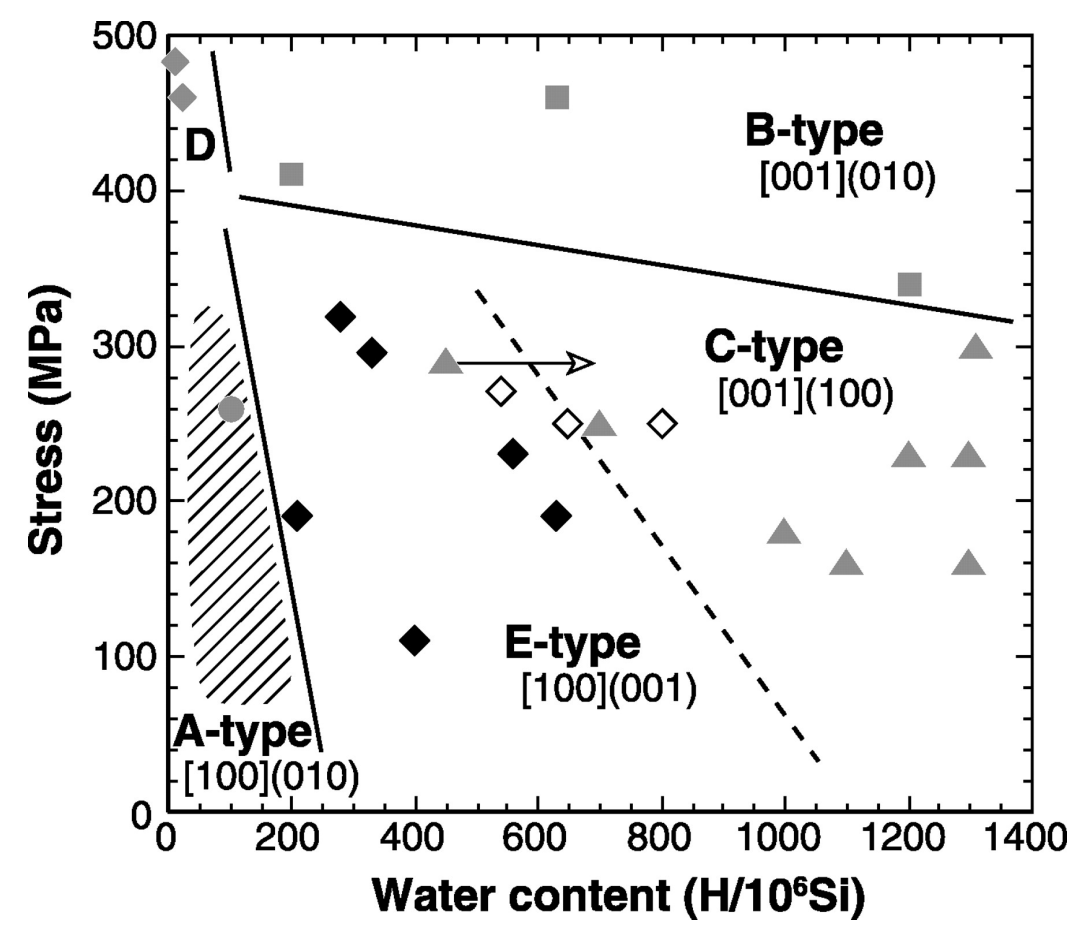

Figure 5.3: Olivine fabric diagram, from Katayama et al. (2004), as function of differential stress (MPa) and water content $\left(\mathrm{H} / 10^{6} \mathrm{Si}\right)$ at high temperatures $(\mathrm{T}=1470-1570 \mathrm{~K})$.

fast azimuth will be subparallel to the flow direction, but type-D fabrics dominate at high stress and low water content while type-E fabrics occur at low stress and moderate water content (Katayama et al., 2004). Below our study region the shear stresses estimated on the slab interface at $110 \mathrm{~km}$ depth are around $170 \mathrm{MPa}$ (Stern et al., 1992), suggesting that type-A, E, and C are the most likely olivine fabrics in our study region (Figure 5.3). We discuss previous interpretations of shear wave splitting results in North Island and suggestions for contributions from different olivine fabrics in the following section.

\subsubsection{Previous upper mantle anisotropy studies}

Several teleseismic shear wave splitting studies have been conducted in North Island (e.g., Gledhill and Stuart, 1996; Marson-Pidgeon et al., 1999; Audoine et al., 2004; Marson-Pidgeon and Savage, 2004; Greve et al., 2008), but none focus on the area across the TRL (Figure 5.4).

Audoine et al. (2004) reported dominant NE-SW fast azimuths from SKS measurements across the northern parts of North Island. They present two possible scenarios for the TVZ and back-arc region of North Island: 1) a control by trench- 
parallel mantle flow (A-type olivine) if the "wet" zone occurs between 90 and 130 $\mathrm{km}$ depth, related to the transpressive character of the plate boundary or 2) trenchparallel anisotropy controlled by the presence of type-B fabric of olivine LPO, if the wet zone is present from 90 to $250 \mathrm{~km}$ depth (Audoine et al., 2004). They suggest that the B-type fabric of olivine can explain their observed trench-parallel fast azimuths if the shear direction is trench perpendicular. However, they note that shear stresses estimated on the slab interface at $110 \mathrm{~km}$ depth are around $170 \mathrm{MPa}$ (Stern et al., 1992), which is significantly less than the value of $270 \mathrm{MPa}$ at which B-type fabric appears in the experiment by Jung and Karato (2001a). For olivine type-A fabric, the LPO of the fast olivine a-axis will align with the extension direction, and is suggested as the source of anisotropy for parts of southern North Island (Gledhill and Stuart, 1996), as well as along the eastern side of North Island (Greve et al., 2008). It has been suggested that trench-parallel mantle flow with type-A olivine beneath the slab can account for most of the observed anisotropy in fore-arc region of central North Island (Greve et al., 2008).

A study by Greve et al. (2008) conducted splitting analyses across the entire Central North Island Passive Seismic Experiment (CNIPSE) line (Figure 5.4; Reyners and Stuart, 2002) by analyzing mantle anisotropy from teleseismic phases. They found that delay times ranged from null measurements to $4.5 \mathrm{~s}$ across central North Island with the largest delay times in the central TVZ and the lowest delay times west of the TVZ. They observed that stations located east of the TVZ exhibited NE-SW fast azimuths with delay times around 2-3 s, with splitting parameters fairly consistent with those observed in southern North Island (Gledhill and Stuart, 1996; Marson-Pidgeon et al., 1999). The trench-parallel fast azimuths east of the TVZ were suggested to be due to the NE-SW lattice-preferred orientation of anisotropic crystals in the mantle due to trench-parallel sub-slab flow, possibly with a contribution from a trench-parallel fossil anisotropy within the slab, with a minor contribution from crustal anisotropy ( $0.3 \mathrm{~s})$ caused by trench-parallel cracks in the overriding plate (Greve et al., 2008). Stations located in the TVZ also exhibited NE-SW fast azimuths but delay times were significantly larger (up to 4.5 s), suggested to be due to the thicker mantle wedge under the TVZ (Greve et al., 2008). Greve et al. (2008) suggest that type-E olivine, in which fast azimuths will be subparallel to the flow direction, can explain the lower stresses and high delay times in the TVZ. However, Greve et al. (2008) also propose that the high delay 
times can be more easily explained by a model of frequency dependent anisotropy in which the alignment of melt bands produces strong anisotropy in the extending TVZ.

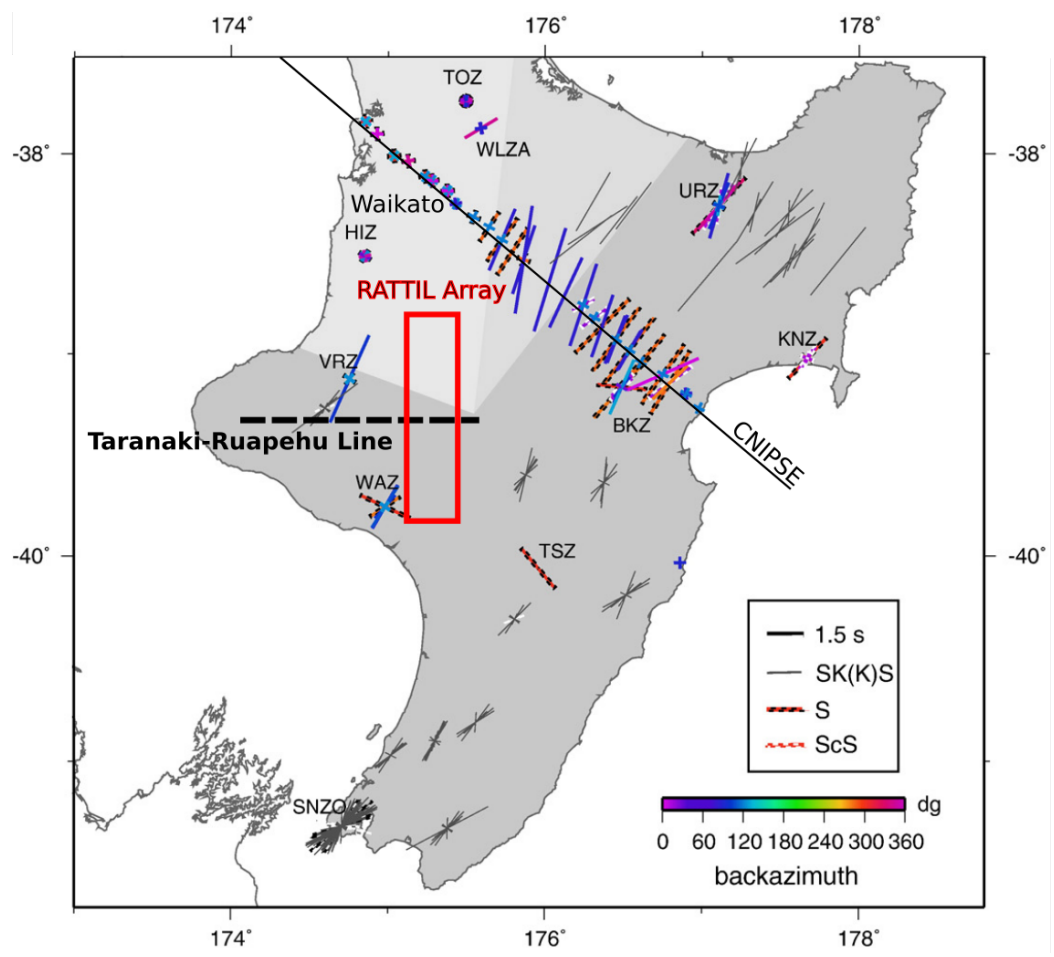

Figure 5.4: Previous splitting measurements (coloured) from teleseismic earthquakes in North Island, New Zealand by Greve et al. (2008) and all earlier (grey) splitting measurements (Audoine et al., 2004; Cochran, 1999; Gledhill and Stuart, 1996; Marson-Pidgeon and Savage, 1997; MarsonPidgeon et al., 1999; Marson-Pidgeon and Savage, 2004) [after Greve et al. (2008)]. The lines represent each fast azimuth and the lengths are scaled according to the delay time. Each line represents a single measurement and is plotted at the corresponding station. The red box highlights the location of stations used in this study. The black dashed line marks the Taranaki-Ruapehu Line. The light shaded area across Waikato marks the region of suggested apparent isotropy (Greve et al., 2008). Our results suggest that the southern boundary is located north of the RATTIL array.

Greve et al. (2008) observed that almost all stations located in western central North Island, west of the TVZ, exhibited only null measurements (no apparent splitting). They suggest that the apparent isotropy observed in western North Island may be due to a vertical return flow under western North Island (Reyners et al., 2006), which can develop hexagonally symmetric anisotropy with a vertical fast axis (Savage, 1999), or from localized small-scale mantle convection. Along with a steeply dipping subducting slab under western North Island, which suggests an end of the mantle wedge dynamics, any anisotropy forming under such conditions would be complex and not resolvable by splitting measurements (i.e., 
resulting in apparent isotropy). These previous studies show a dramatic change from highly coherent NE-SW fast azimuths suggested to start south of the TRL and incoherent anisotropy north of the TRL (Greve et al., 2008; Audoine et al., 2004), which is suggested to be caused by the sheared thick lithosphere to the south and an absence of the lithosphere to the north, however the exact location of this transition has not yet been properly identified. The light shaded area in Figure 5.4 across Waikato marks the region of previously suggested apparent isotropy (Greve et al., 2008).

\subsection{Data and Methods}

The Ruapehu And Taranaki Teleseismic Imaging Line (RATTIL) temporary landbased seismic array consisted of 14 broadband seismometers positioned northsouth across the TRL, which operated between November 2012 and February 2014 (Dimech, 2016). Of these 14 stations, four experienced instrument problems and for that reason we did not use them for our analyses. The remaining 10 stations, shown in Figure 5.1, acquired sufficient data for further analyses. However, the time and duration of the station deployments varied significantly (Figure 5.5). Using United Stated Geological Survey (USGS) earthquake locations we analyzed 726 teleseismic events spanning the time of the RATTIL deployment, between $85^{\circ}$ and $180^{\circ}$ from each station, with magnitudes ranging from Mw 5 to 8.3 and depths ranging from 0-624 km (Figure 5.6).

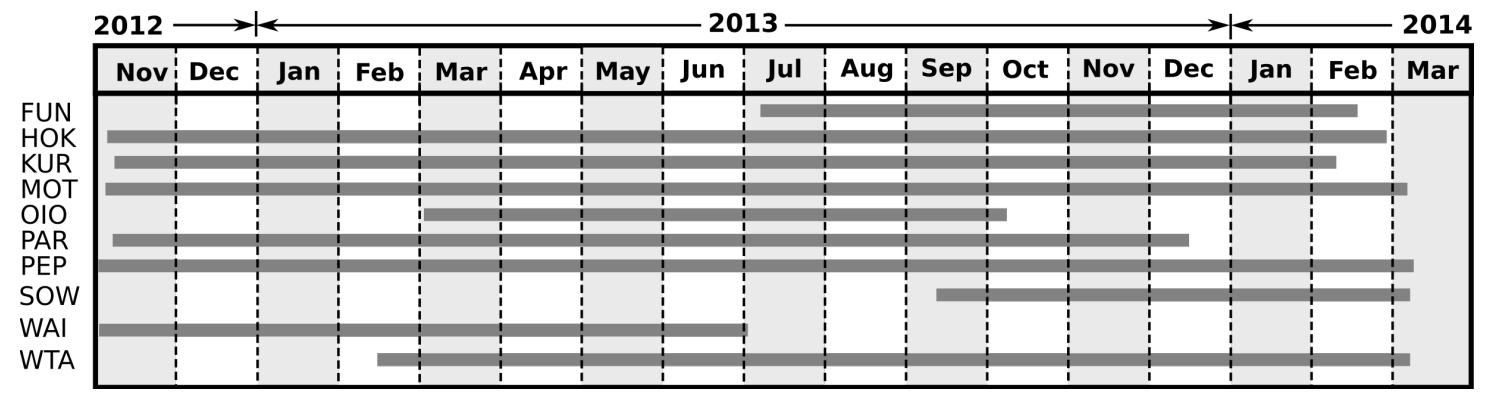

Figure 5.5: Deployment timeline for the 10 RATTIL stations used in this study [after Dimech (2016)].

\subsubsection{SplitRacer}

We used the SplitRacer software package by Reiss and Rümpker (2017) to analyze teleseismic SWS by using the minimum transverse energy technique (Silver 

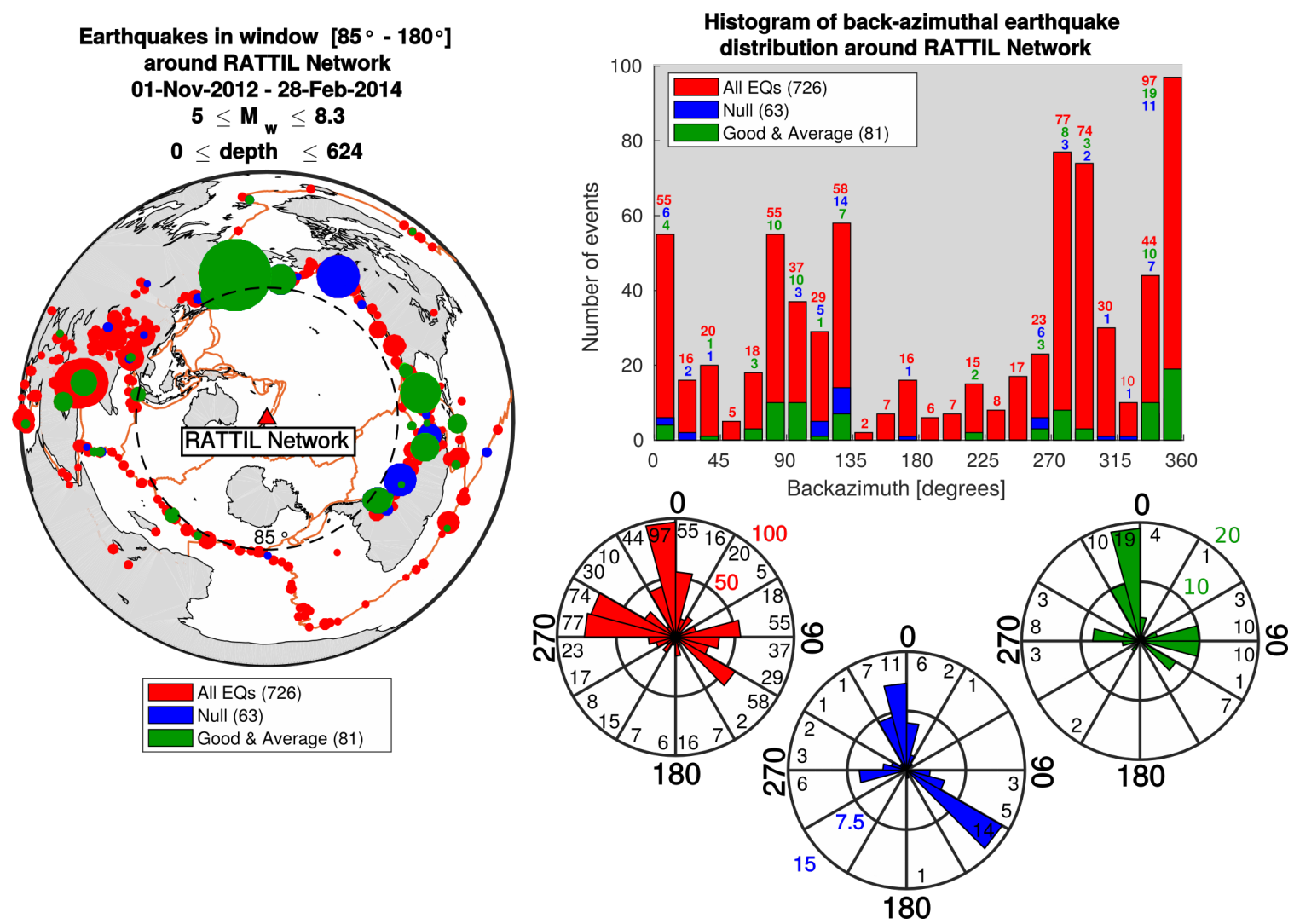

Figure 5.6: Distribution statistics of earthquakes recorded between $85^{\circ}-180^{\circ}$ from the RATTIL network. Left panel shows azimuthal map of earthquake locations. All earthquakes analyzed in this study are marked by red circles. Green circles mark events which gave "Good and Average" SWS results in SplitRacer. Blue circles mark events which gave null results. Top right panel is a histogram showing the backazimuthal distribution of earthquakes. Bottom right panel is the backazimuthal distribution displayed as rose diagrams for all earthquakes (red), earthquakes which provided null measurements (blue), and events which provided good and average grade measurements (green).

and Chan, 1991). We analyzed data from teleseismic earthquakes occurring between $85^{\circ}-180^{\circ}$ distance from the RATTIL array. The chosen distance allowed us to include all core phases, such as SKS, SKKS, SKIKS, PKIKS and PKS (called XKS phases), which were used in this study. A minimum event magnitude of Mw 5 was used to find suitable events.

First, we filtered the data by applying a bandpass filter of 11-20 s. This frequency range allowed for the greatest reduction in noise while still preserving the XKS phases. We chose a signal-to-noise ratio (SNR) of 1.5 to automatically select suitable phases of an event. XKS phases were identified using the IASP91 velocity model in Taup (Crotwell et al., 1999). Selected phases were manually inspected 
during pre-processing and time windows were adjusted, if necessary. We used the predefined 50 randomly-chosen different time windows for analysis, allowing for a thorough statistical analysis of the results (Reiss and Rümpker, 2017).

Three-component broadband seismometers record horizontal velocities on north and east components. Each waveform was respectively transformed from a VerticalEast-North (ZEN) coordinate system to an LQT coordinate system using the IASP91 velocity model in Taup (Crotwell et al., 1999), in which the L-component points along the raypath, the Q-component points towards the earthquake, and the Tcomponent is perpendicular to the ray plane. Next, in the SplitRacer software package, the transverse component minimization method uses a grid search approach over all fast azimuth and delay time values that best minimizes the energy on the transverse component (Figure 5.7). The method iterates over all possible values of the fast azimuth (in $1^{\circ}$ increments) and delay time (in $0.1 \mathrm{~s}$ increments with maximum value of $4 \mathrm{~s}$ ). We checked the linearity of the corrected particle motion manually for each measurement. In general, if energy is present on the transverse component and an elliptical particle motion is observed it indicates the possible presence of anisotropy (Silver and Chan, 1991). If the anisotropy correction does not result to the linearization of particle motion and/or the energy reduction was less than $50 \%$ then the event was marked by SplitRacer as "bad". A "good" measurement should have a clear phase onset on both the pre-rotated radial and transverse components, with near elliptical particle motion, by a reduction in energy on the transverse component after rotating to QTL, a large SNR, and small 95\% confidence intervals in fast azimuths and delay time. An "average" grade was used for measurements which appeared slightly noisier than that of a "good" measurement (Reiss and Rümpker, 2017). The absence of energy on the transverse component is considered a "null" measurement. Each measurements is marked as either "good", "average", "bad", or "null" and all but "bad" measurements are used for further analyses.

In addition to the single phase splitting measurements, a joint splitting inversion is applied by using all waveforms characterized as either "good", "average" or "null". The joint splitting inversion stacks the energy grid of each phase, and the smallest energy value yields a single fast azimuth and delay time value that best reduces the transverse energy on all phases simultaneously for each station, similar to the approach by Wolfe and Silver (1998). This inversion allows for the reduction 
a)

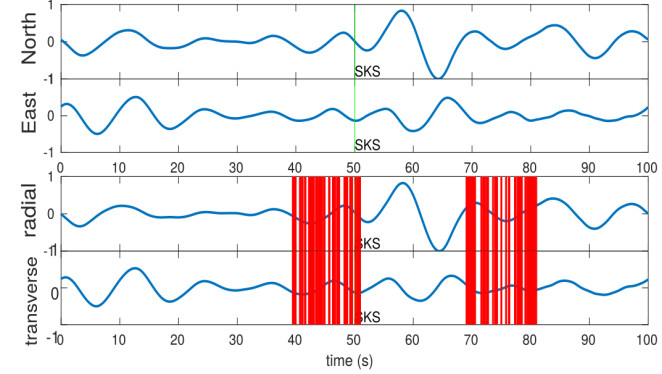

d)

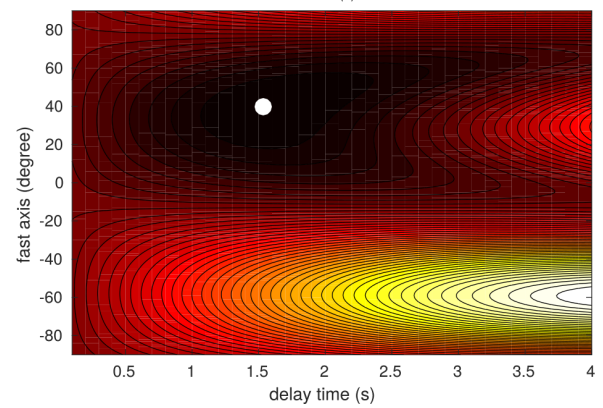

b)

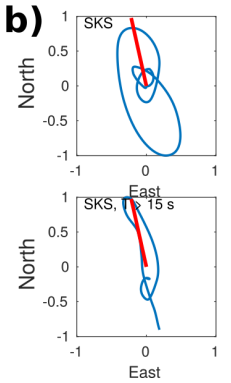

e)

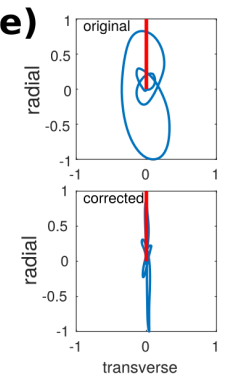

c)
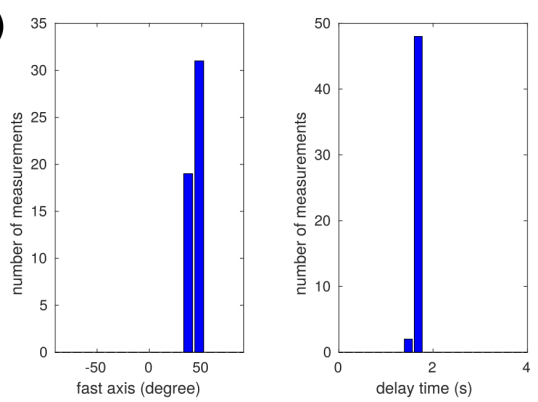

f)

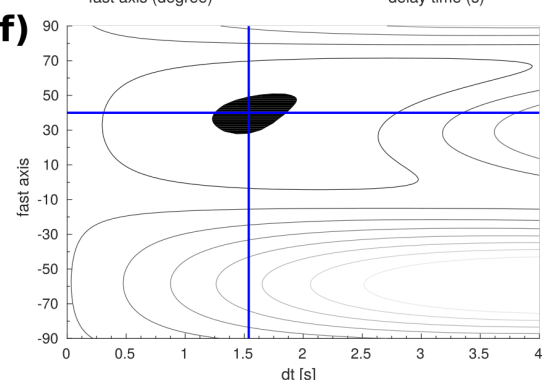

Figure 5.7: Example of a "good" shear wave splitting measurement using SplitRacer (Reiss and Rümpker, 2017) at station HOK (24th of May, 2013). a) North/East and radial/transverse components. The SKS phase arrival is marked by the green line. 50 different time windows used are shown by red lines. b) Particle motion for the SKS phase time window. The theoretical backazimuth is marked by the red bar. c) Histogram of all measurements. d) Average energy grid with average splitting parameters marked with a white dot. e) Original and corrected particle motion. f) $95 \%$ confidence intervals on top of the energy grid. The blue cross marks the average splitting parameters.

of noise and increases the robustness of the measurements (Reiss and Rümpker, 2017). To compare the joint splitting inversion to single phase measurement we also calculate the circular mean of the fast azimuths and the mean delay time for each station.

\subsection{Results}

We applied the transverse minimization method to each individual XKS phase at each station from the RATTIL array (Reiss and Rümpker, 2017). For a bandpass filter of $11-50 \mathrm{~s}$, a total of 144 measurements were made on 10 RATTIL stations. 81 measurements were graded as "good" and "average", while 63 measurements were determined to be "Nulls" (Appendix D, Table D.1). A map, histogram, and rose plot showing the distribution of the earthquakes producing these measurements can be found in Figure 5.6. The XKS phases which provided "good" and "average" measurements consisted of 58 SKS, 16 SKKS, 4 SKIKS, 2 PKIKS and 1 PKS phase 
Table 5.1: $\Phi$ and delay time for individual RATTIL stations.

\begin{tabular}{|c|c|c|c|c|c|c|c|c|c|c|c|c|}
\hline Station & Lat & Lon & $\mathrm{N}$ & N Nulls & JS $\phi$ & $\mathrm{JS} \delta t$ & JS $\phi$ error & JS $\delta t$ error & $\mu(\phi)$ & $\phi \mathrm{SE}$ & Mean $\delta t$ & $\delta t \mathrm{SE}$ \\
\hline PEP & -38.81 & 175.35 & 18 & 7 & $50^{\circ}$ & $1.0 \mathrm{~s}$ & {$\left[30^{\circ}, 67^{\circ}\right]$} & {$[0.4 \mathrm{~s}, 1.6 \mathrm{~s}]$} & $36^{\circ}$ & $21^{\circ}$ & $2.2 \mathrm{~s}$ & $0.29 \mathrm{~s}$ \\
\hline FUN & -38.96 & 175.36 & 18 & 12 & $55^{\circ}$ & $1.4 \mathrm{~s}$ & {$\left[47^{\circ}, 63^{\circ}\right]$} & {$[1.0 \mathrm{~s}, 1.8 \mathrm{~s}]$} & $45^{\circ}$ & $20^{\circ}$ & $2.7 \mathrm{~s}$ & $0.27 \mathrm{~s}$ \\
\hline $\mathrm{OIO}$ & -39.11 & 175.07 & 7 & 2 & $47^{\circ}$ & $1.1 \mathrm{~s}$ & {$\left[34^{\circ}, 60^{\circ}\right]$} & {$[0.6 \mathrm{~s}, 1.6 \mathrm{~s}]$} & $60^{\circ}$ & $53^{\circ}$ & $1.5 \mathrm{~s}$ & $0.29 \mathrm{~s}$ \\
\hline KUR & -39.18 & 175.29 & 17 & 10 & $29^{\circ}$ & $1.2 \mathrm{~s}$ & {$\left[22^{\circ}, 37^{\circ}\right]$} & {$[0.9 \mathrm{~s}, 1.5 \mathrm{~s}]$} & $44^{\circ}$ & $20^{\circ}$ & $2.1 \mathrm{~s}$ & $0.41 \mathrm{~s}$ \\
\hline MOT & -39.34 & 175.28 & 10 & 5 & $26^{\circ}$ & $1.2 \mathrm{~s}$ & {$\left[20^{\circ}, 32^{\circ}\right]$} & {$[0.8 \mathrm{~s}, 1.6 \mathrm{~s}]$} & $39^{\circ}$ & $11^{\circ}$ & $1.9 \mathrm{~s}$ & $0.42 \mathrm{~s}$ \\
\hline SOW & -39.37 & 175.28 & 6 & 4 & $-41^{\circ}$ & $1.4 \mathrm{~s}$ & {$\left[-56^{\circ},-24^{\circ}\right]$} & {$[0.7 \mathrm{~s}, 2.1 \mathrm{~s}]$} & $-9.5^{\circ}$ & $23^{\circ}$ & $2.3 \mathrm{~s}$ & $0.21 \mathrm{~s}$ \\
\hline $\mathrm{HOK}$ & -39.45 & 175.26 & 31 & 11 & $31^{\circ}$ & $1.3 \mathrm{~s}$ & {$\left[26^{\circ}, 37^{\circ}\right]$} & {$[1.1 \mathrm{~s}, 1.5 \mathrm{~s}]$} & $45^{\circ}$ & $14^{\circ}$ & $1.9 \mathrm{~s}$ & $0.17 \mathrm{~s}$ \\
\hline WAI & -39.5 & 175.19 & 8 & 4 & $21^{\circ}$ & $1.3 \mathrm{~s}$ & {$\left[13^{\circ}, 2^{\circ}\right]$} & {$[1.0 \mathrm{~s}, 1.6 \mathrm{~s}]$} & $33^{\circ}$ & $16^{\circ}$ & $1.6 \mathrm{~s}$ & $0.23 \mathrm{~s}$ \\
\hline WTA & -39.55 & 174.8 & 7 & 3 & $47^{\circ}$ & $1.3 \mathrm{~s}$ & {$\left[41^{\circ}, 53^{\circ}\right]$} & {$[1.1 \mathrm{~s}, 1.6 \mathrm{~s}]$} & $46^{\circ}$ & $5^{\circ}$ & $1.5 \mathrm{~s}$ & $0.18 \mathrm{~s}$ \\
\hline PAR & -39.73 & 175.26 & 22 & 5 & $33^{\circ}$ & $0.7 \mathrm{~s}$ & {$\left[12^{\circ}, 54^{\circ}\right]$} & {$[0.3 \mathrm{~s}, 1.2 \mathrm{~s}]$} & $41^{\circ}$ & $6^{\circ}$ & $1.6 \mathrm{~s}$ & $0.11 \mathrm{~s}$ \\
\hline - & - & - & 144 & 63 & $36.1^{\circ} \pm 5^{\circ}$ & $1.22 \mathrm{~s} \pm 0.07 \mathrm{~s}$ & - & - & $43^{\circ}$ & $5^{\circ}$ & $1.9 \mathrm{~s}$ & $0.11 \mathrm{~s}$ \\
\hline
\end{tabular}

Note: N: Total number of measurements. N Null: Total number of null measurements. JS: Joint Splitting Inversion.

$\mu(\phi)$ : circular mean of the fast polarization azimuth (relative to North). SE: Standard error. The last row provides totals and averages using all of the stations. 

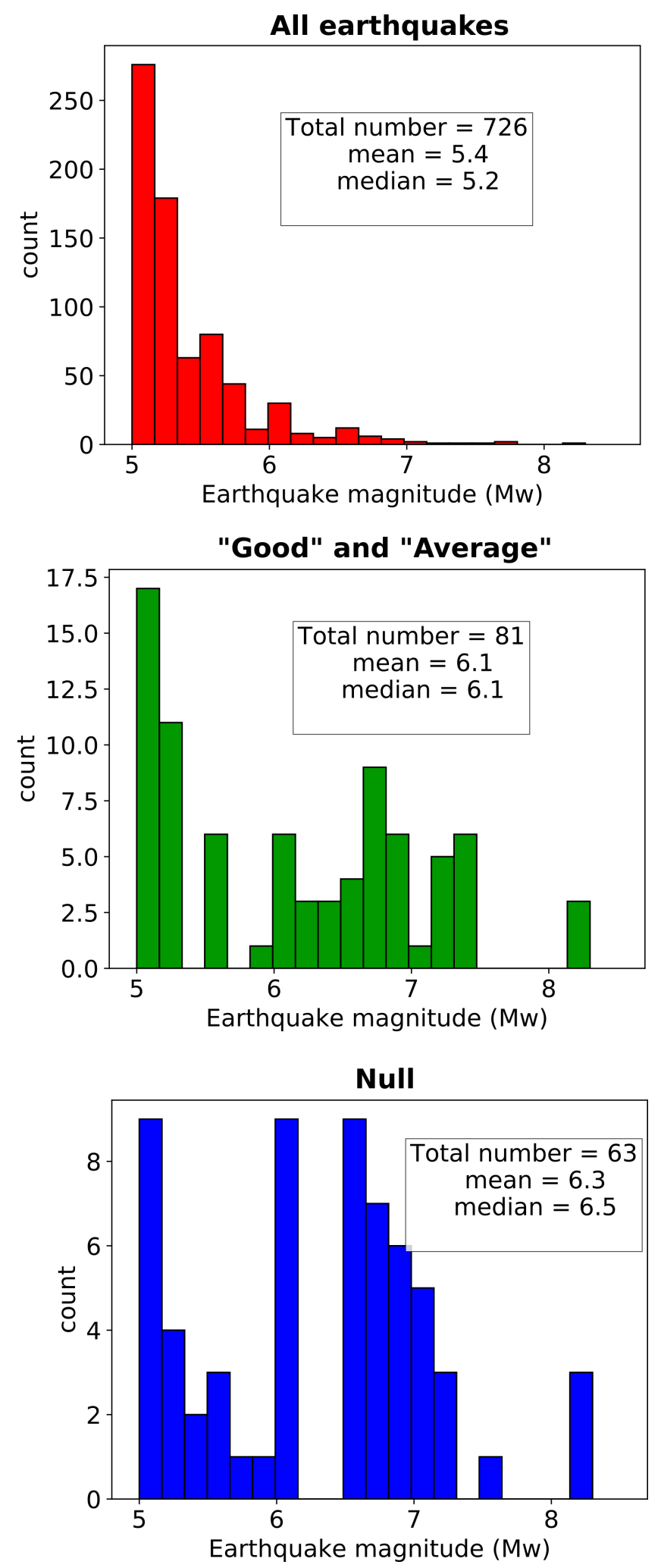

Figure 5.8: Histogram of earthquake magnitudes which provided a "good" and "average" grade measurement using SplitRacer (Reiss and Rümpker, 2017). 
(Appendix D, Table D.1). By analyzing the histogram of magnitudes (Figure 5.8) we show that a Mw 5 cut-off limit provides significantly more measurements (35 "good" and "average" measurements from earthquakes with magnitudes between 5 and 6) than the commonly used cut-off of Mw 6. We calculate the circular mean of the fast azimuth, mean delay time, and standard errors for measurements from earthquakes with magnitudes ranging from Mw 5-6 (fast azimuth of $48.2^{\circ} \pm 10.8^{\circ}$ and delay time of $1.89 \mathrm{~s} \pm 0.15 \mathrm{~s}$ ) and for earthquakes with magnitudes from Mw 6 and above (fast azimuth of $40.7^{\circ} \pm 5.1^{\circ}$ and delay time of $1.92 \mathrm{~s} \pm 0.1 \mathrm{~s}$ ). We observe little variation in fast azimuths and delay times from earthquakes with magnitudes Mw 5-6 versus 6 and above. We suggest that a cut-off of Mw 5 should be considered for future teleseismic SWS studies in this region.

We applied the joint splitting inversion, which uses all XKS phases at each station which provided a "good", "average", and "null" result. We examine the reduction of energy on the transverse components and the corrected particle motion and find this approach suitable. We present the circular mean of the fast azimuths, the mean delay times and the results from the joint splitting inversion for each individual station in Table 5.1. The resulting fast azimuths from the joint splitting inversion are similar to the circular averages for each station (Figure 5.9, Table 5.1); however, the delay times show greater variation between the two averaging techniques. The average fast azimuth and standard error for all combined stations is $42.5^{\circ} \pm 5^{\circ}$ with a delay time of $1.9 \mathrm{~s} \pm 0.11 \mathrm{~s}$. The average from the joint splitting inversion is $36.1^{\circ} \pm 8^{\circ}$ with a delay time of $1.22 \mathrm{~s} \pm 0.07 \mathrm{~s}$.

We plot the fast azimuths for the circular mean and from the joint splitting inversion in Figure 5.9. Most individual stations exhibit a dominant NE-SW direction (Figure 5.9). We observe little variability in station fast azimuths in both the circular mean and joint splitting inversion (Figure 5.9, Table 5.1). Station SOW, located just south of the TRL exhibits a NNW-SSE fast azimuth; however, due to this stations shorter deployment period, lasting only $\sim 6$ months (Figure 5.5), there were significantly fewer processed earthquakes. For this reason the fast azimuth average at station SOW is based on two measurements. Additionally, the measurements at station SOW belong to earthquakes which do not provide measurements at any other station, so we do not interpret the results at this station in great detail.

We plot the mean and joint splitting inversion delay times in Figure 5.10. The 


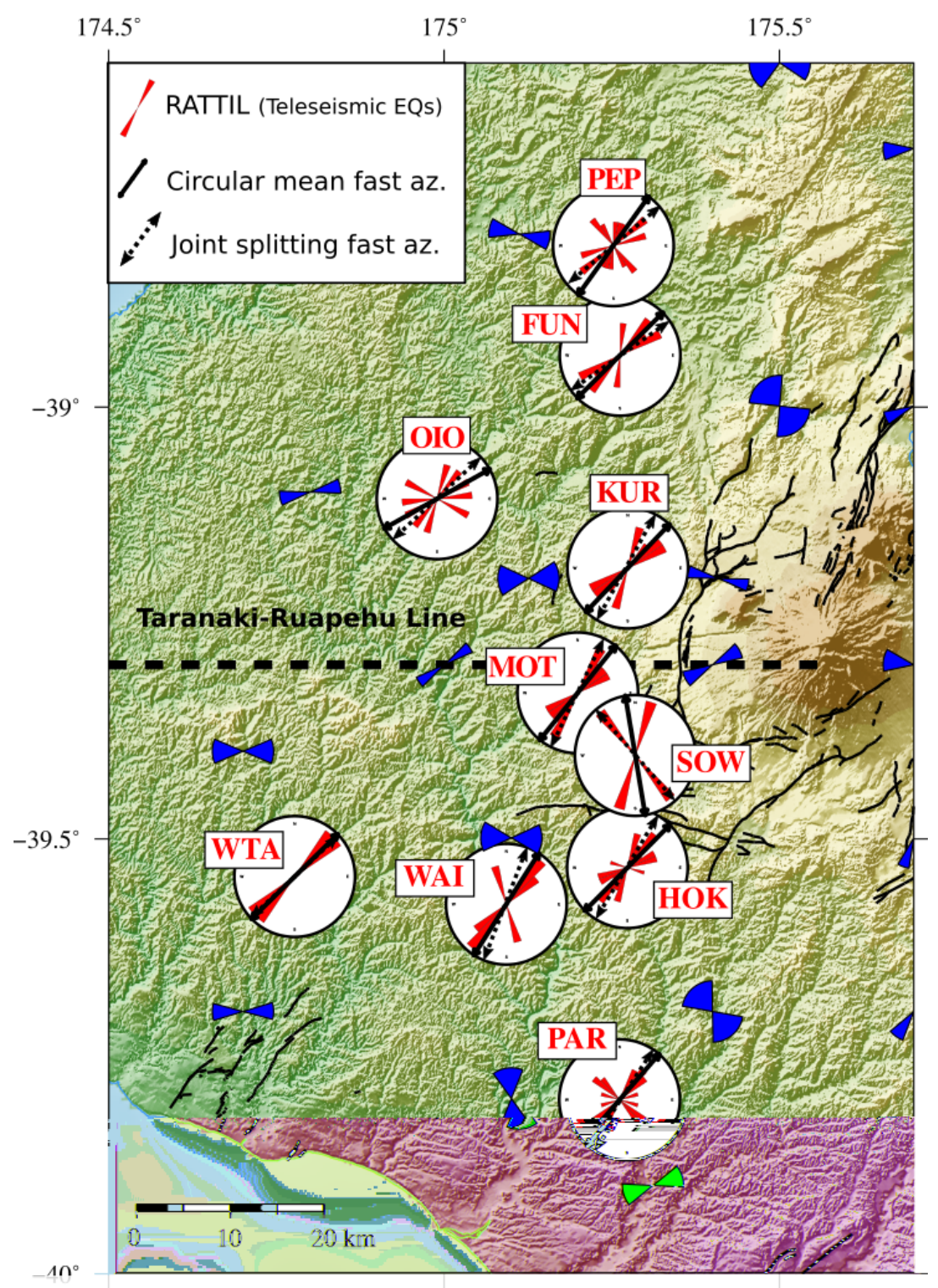

Figure 5.9: Rose diagrams of SWS fast azimuths at each station. Teleseismic events were used to measure upper mantle anisotropy on the RATTIL stations (red). The length of the sectors are proportional to the square root of the number of measurements in each sector. The black arrows on each rose plot are the mean fast azimuths. The dotted black arrow is the fast azimuth from the joint splitting inversion. Black lines mark active faults from Langridge et al. (2016). $S_{H}$ max directions derived from focal mechanisms are shown as blue bow-ties (Townend et al., 2012).

mean delay times at individual stations range from 1.5-2.7 s, while joint splitting inversion delay times range from 0.7-1.4 s. The joint splitting delay times are considerably lower than the mean delay times, which is likely due to the inclusion of null measurements in the joint splitting inversion, which act to lower the delay time (Wolfe and Silver, 1998). We observe generally larger mean delay times on individual stations that are located north of the TRL ( $2.1 \mathrm{~s} \pm 0.25 \mathrm{~s})$, compared 


\section{Delay Times}

(A) Teleseismic (JS inversion)

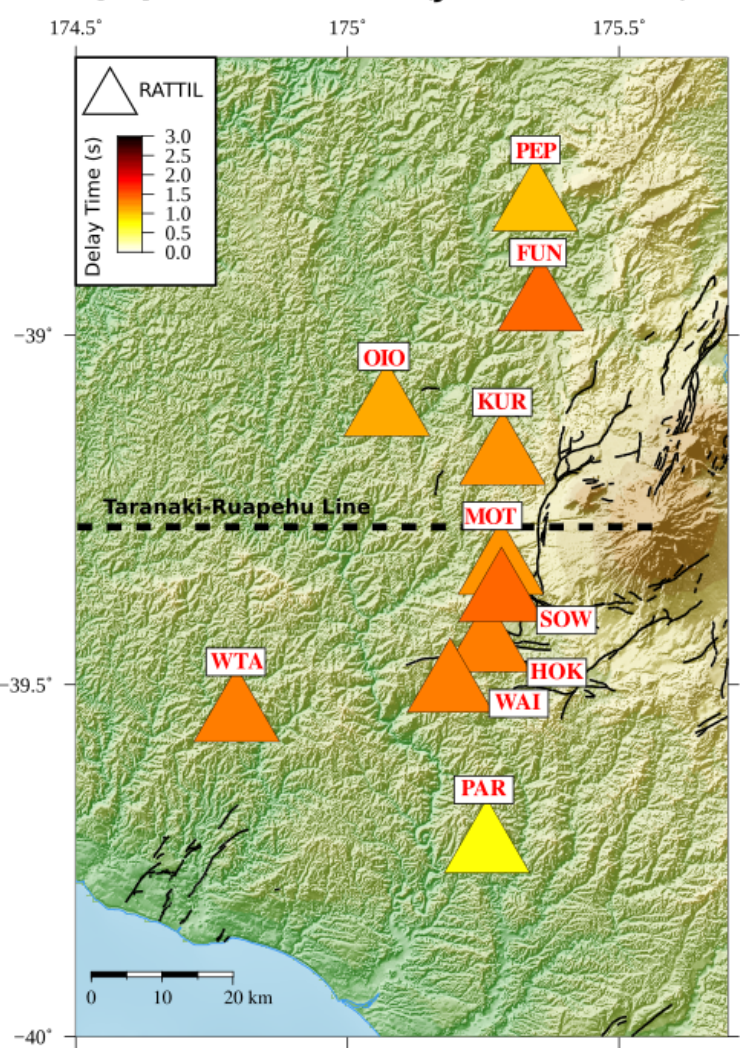

(B) Teleseismic (mean)

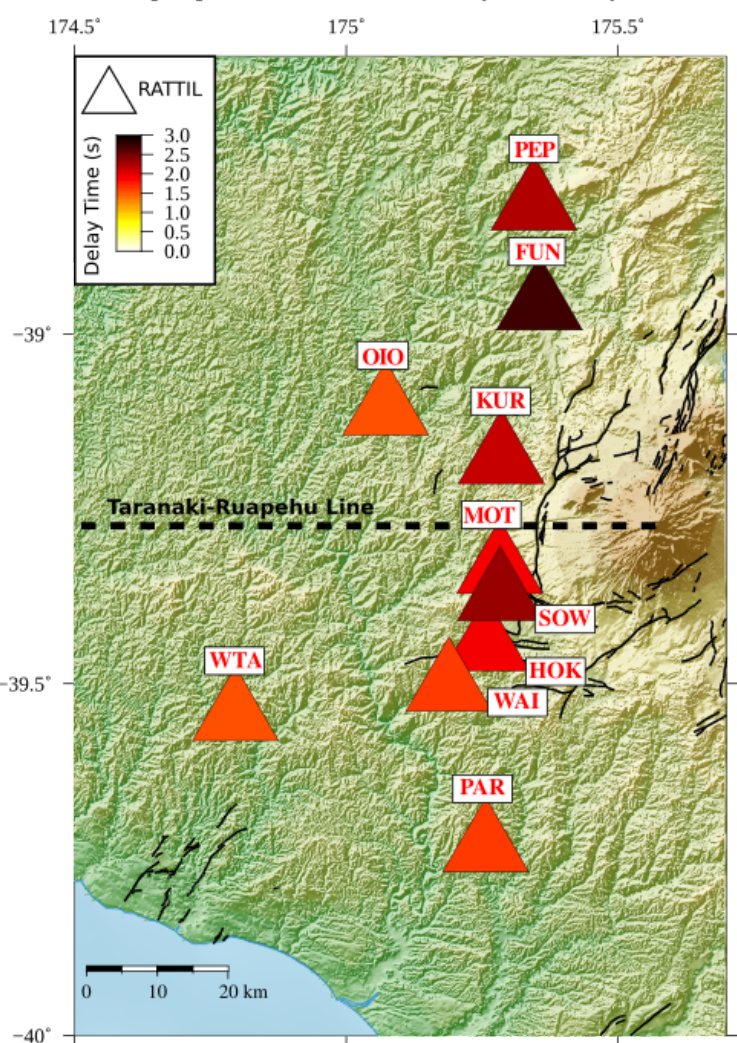

Figure 5.10: Delay times plotted on the stations at which measurements were made. Teleseismic events were used to measure upper mantle anisotropy on the RATTIL stations using a (A) joint splitting inversion and (B) mean. Black lines mark active faults from Langridge et al. (2016).

to south of the TRL (1.8 s $\pm 0.12 \mathrm{~s})$. However, delay times from the joint splitting inversion do not show significant variations.

We examine measurements from the same earthquakes at different stations (Appendix D, Table D.2). A magnitude 7.4 earthquake occurring on November 7th 2012 offshore the west coast of Guatemala provided the most measurements from a single earthquake, recorded on stations HOK, KUR, MOT, PAR, PEP, and WAI (Appendix D, Table D.2). These stations all provided "good" measurements from SKS phases. To supplement these measurements, we perform SWS using SplitRacer on GeoNet stations WAZ, VRZ, and FWVZ for this event (Figure 5.1). Figure 5.11 shows a record section of the waveforms for this event. For this individual event, we observe an increasing delay time for stations located closest to the TRL. We visualize this change in delay times across the TRL in Figure 5.12. 


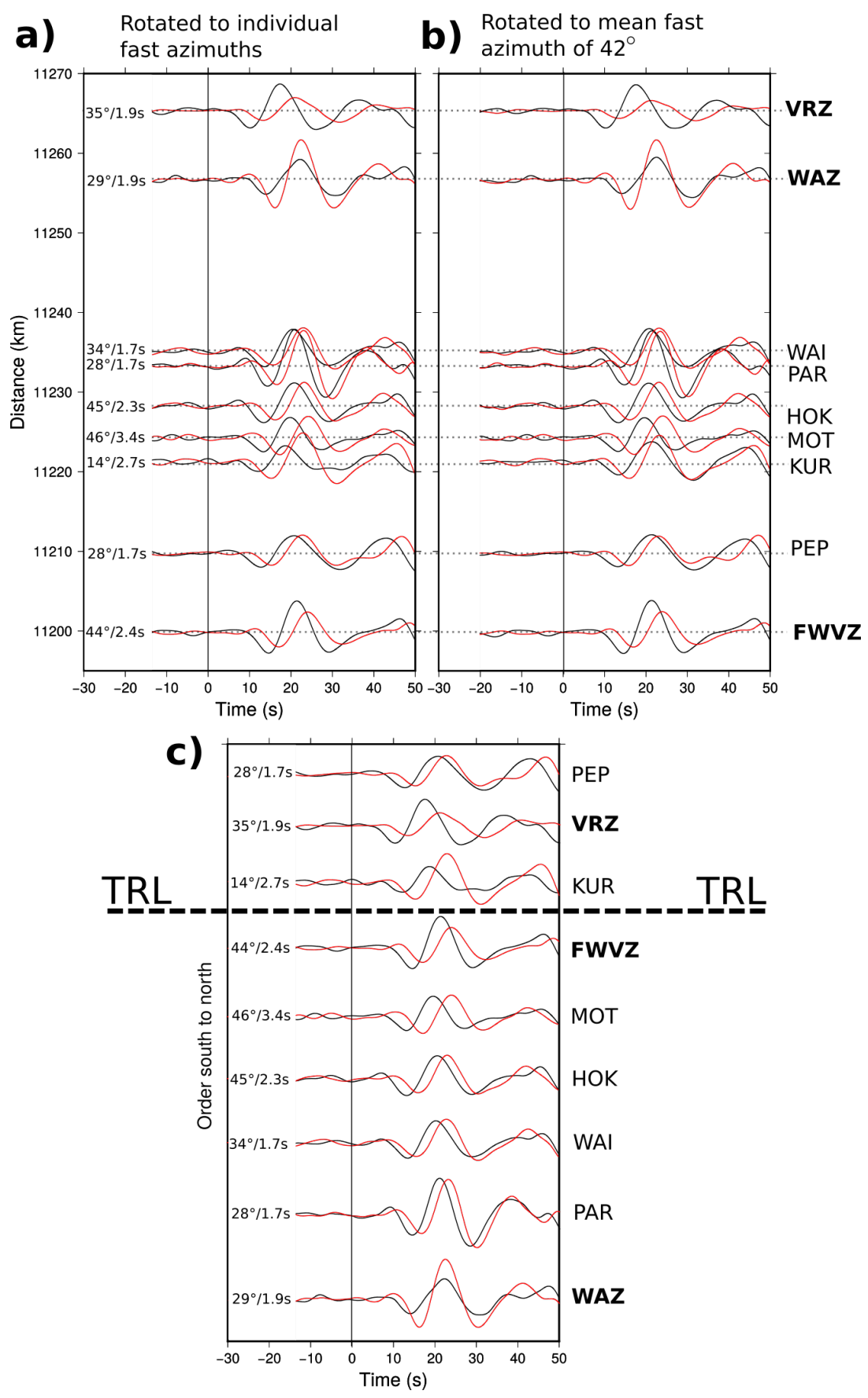

Figure 5.11: Record section showing filtered (bandpass 0.05-0.1 Hz) for event 07-Nov-2012, $\mathrm{Mw}=7.4, \mathrm{BAZ}=81^{\circ}$ to illustrate changes in delay time across the TRL. Phases are lined up based on their expected arrival times from IASP91 velocity model in Taup (Crotwell et al., 1999). A) rotated into their individual fast (black) and slow (red) azimuths and positioned based on distance from source. The events are plotted on the same absolute amplitude scale. B) rotated into their all stations mean average of $42^{\circ}$. C) rotated into their individual fast and slow azimuths and positioned based on the station order across the TRL (south to north). Horizontal dashed line marks the position of the TRL between stations KUR and FWVZ. 


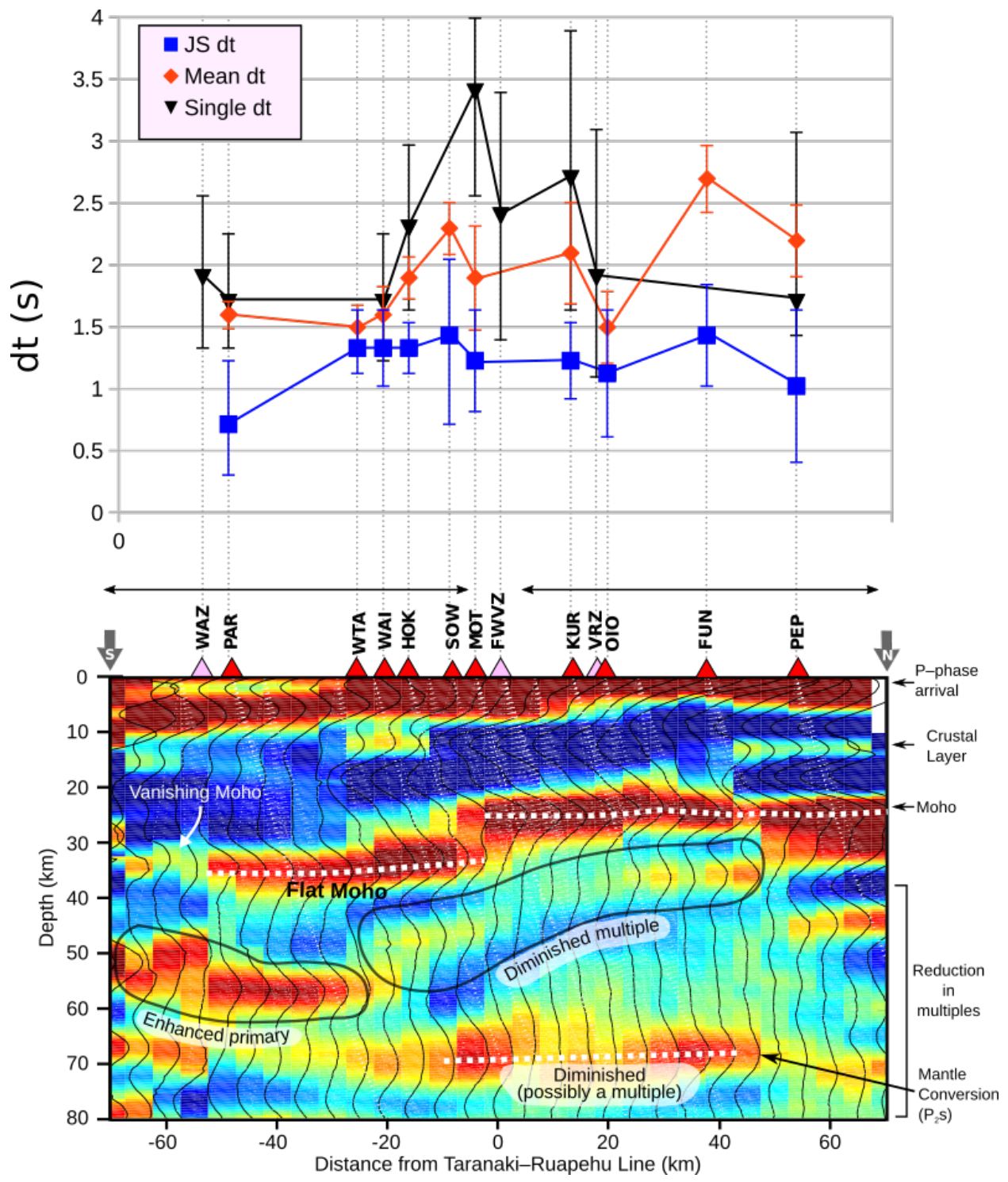

Figure 5.12: Cross section along the RATTIL line illustrating changes in delay times, dt, for an individual event (black) from Figure 5.11, mean station delay times (orange), and delay times from joint splitting inversion (blue) plotted at the station at which measurements were made (from Table 5.1). Crustal structure profile from Dimech et al. (2017), showing location of step in the Moho.

\subsection{Discussion}

Fast azimuth averages from teleseismic SWS recorded at stations on the RATTIL array exhibit dominant NE-SW (trench-parallel) directions across the TRL (Figure 5.9 and Table 5.1). Our station averaged fast azimuths for the RATTIL array is $42.5^{\circ}$ $\pm 5^{\circ}$ with a delay time of $1.9 \mathrm{~s} \pm 0.11 \mathrm{~s}$. The average from the joint splitting inversion is similar with a fast azimuth of $36.1^{\circ} \pm 8^{\circ}$ and a delay time of $1.22 \mathrm{~s} \pm 0.07 \mathrm{~s}$. Many teleseismic shear wave splitting studies across North Island, New Zealand 
have shown consistent fast azimuths subparallel to the trench (e.g., Cochran, 1999; Gledhill and Stuart, 1996; Marson-Pidgeon and Savage, 1997; Marson-Pidgeon et al., 1999; Marson-Pidgeon and Savage, 2004), with greater variation from measurements from local earthquakes (e.g., Unglert, 2011; Audoine et al., 2004; IllsleyKemp et al., 2019), due to stronger lateral variations in the crust. We examine our measurements in context of previous studies.

The RATTIL array is situated above the mantle wedge. The main sources of lithospheric mantle wedge anisotropy are either aligned melt filled cracks (e.g., Ando et al., 1983), the LPO of olivine due to strain or anisotropy in the slab.

In the case that melt filled cracks control the anisotropy in the mantle wedge, the fast azimuths should be parallel to the maximum principle stress direction. The orientation of the stress field across New Zealand has been determined from the inversion of earthquake focal mechanisms by Townend et al. (2012) (Figure 5.9). Using a clustering algorithm, these authors calculate $S_{H \max }$ directions using clusters of focal mechanisms from earthquakes as deep as $400 \mathrm{~km}$ in our study region. The orientation of $S_{H} \max$ across the TRL is generally E-W, with more variation in directions closer to Mt. Ruapehu, as well as to the south, near station PAR (Figure 5.9). Assuming that this stress field exists in the mantle wedge, the fast azimuths from stress-induced anisotropy should be E-W, which is inconsistent with our observed measurements.

If we assume that anisotropy is caused by the LPO of olivine with type-A fabric, due to strain or flow, the observed NE-SW fast azimuths would suggest that the dominant shear deformation is associated with oblique convergence and that flow is in a trench-parallel direction. The same interpretation was made by MarsonPidgeon et al. (1999), who observed NE-SW fast azimuths across central North Island. Similarly, Gledhill and Stuart (1996) observe an average fast azimuth of $28^{\circ}$ from SKS measurements in southern North Island, and suggest trench-parallel flow in the mantle below the subducting slab. A study of SKS shear wave splitting in the East Cape region has reported an average fast azimuth $\left(26^{\circ}\right)$, subparallel to the trench, with an average delay time of $2.1 \mathrm{~s}$ (Cochran, 1999), and they suggest a combination of trench-parallel fossil anisotropy in the slab and trench-parallel flow in the subslab mantle may explain these directions. This is the most likely scenario, as large strain by simple shear will orient the olivine a-axes parallel to 
the flow direction and trench-parallel fossil anisotropy in the slab can contribute to the observed anisotropy (e.g., Marson-Pidgeon et al., 1999). The rays travelling to the RATTIL stations travel through both the mantle wedge and subslab mantle, however stations located in East Cape (Audoine et al., 2004) and southern North Island (Marson-Pidgeon et al., 1999) observe similar fast azimuths from teleseismic rays travelling through only the subslab mantle and subducting plate, suggesting that similar fast azimuths are observed in the mantle wedge and subslab mantle (Figure 5.13).

Conventional models of mantle flow in subduction zones suggest that the flow is parallel to the downdip slab direction, both above and below the plate (Ribe, 1989), due to the relative plate motion between the subducting and overriding plates (Figure 5.13). In this model the a-axes of olivine would be parallel to the downdip slab direction. However, in our case the NE-SW fast azimuths suggest that flow is trench-parallel, which has been similarly interpreted by previous studies in this region (Figure 5.13; Marson-Pidgeon et al., 1999), with the possibility of contribution from trench-parallel fossil anisotropy in the slab. In order to determine the contribution of anisotropy in the crust, mantle wedge, slab, and subslab mantle we would need to examine SWS on local earthquakes.

Below our study region the shear stresses estimated on the slab interface at 110 $\mathrm{km}$ depth are around $170 \mathrm{MPa}$ (Stern et al., 1992), suggesting that type-A, E, and $\mathrm{C}$ are the most likely olivine fabrics in our study region (Figure 5.3), while type-D and B fabrics are observed at significantly higher stresses (>350 MPa). Greve et al. (2008) suggest that type-E olivine, in which fast azimuths will be subparallel to the flow direction, can explain the high delay times (as high as $4.5 \mathrm{~s}$ ) they observe in the TVZ. Type-E olivine occurs in conditions of high water content and could result in such large delay times; however our delay times of $1.9 \mathrm{~s} \pm 0.11$ s are significantly lower, contradicting this hypothesis. Type-C olivine occurs with higher water content compared to type-E olivine, requiring even higher delay times than those observed in the TVZ. We suggest that our results are best explained by typeA olivine with flow in a trench-parallel direction and the possibility of contribution from trench-parallel fossil anisotropy in the slab.

Greve et al. (2008) observe mainly null measurements along the WCNIPSE line, in the Waikato region (Figure 5.4). They suggest that the apparent isotropy ob- 

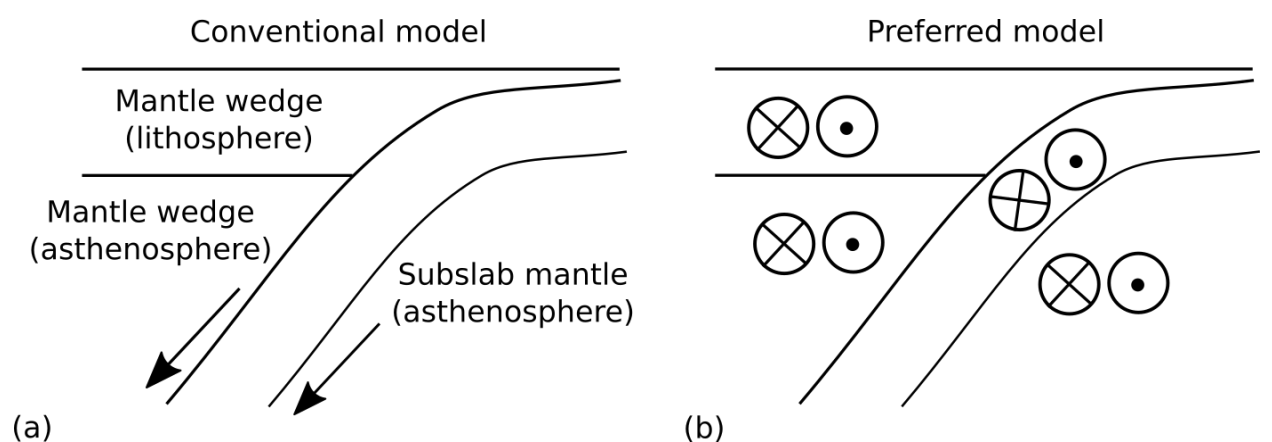

Figure 5.13: Schematic diagram of a subduction zone, comparing the conventional model of mantle flow with our preferred model [from Marson-Pidgeon et al. (1999)]. (A) Conventional model predicts that mantle flow is parallel to the downdip slab direction both above and below the slab (Ribe, 1989). (B) Our measurements indicate trench-parallel fast azimuths in the mantle wedge. Previous SWS measurements in eastern (Audoine et al., 2004) and southern (Marson-Pidgeon et al., 1999) North Island also suggest trench-parallel flow in the subslab mantle. These results suggest trenchparallel flow both above and below the slab. Anisotropy in the slab is probably caused by fossil mineral alignment (Marson-Pidgeon et al., 1999).

served in western North Island may be due to a vertical return flow under western North Island (Reyners et al., 2006), which can develop hexagonally symmetric anisotropy with a vertical fast axis (Savage, 1999), or from localized small-scale mantle convection. Greve et al. (2008) approximate the transition point to apparent isotropy observed in the north to start north of GeoNet station VRZ $\left(39.1^{\circ} \mathrm{S}\right)$, for which they observed mainly null measurements with a single measurement producing a high delay time. A study by Marson-Pidgeon et al. (1999) found trench-parallel fast azimuths on a station $20 \mathrm{~km}$ southwest of station VRZ, from a temporary deployment. Our measurements suggest that the TRL region does not experience the same apparent isotropy that has been observed further north. Our results suggest that the transition point to apparent isotropy may be significantly further north than has been previously suggested. Of the stations used in this study, station PEP is located furthest north $\left(38.8^{\circ} \mathrm{S}\right)$ and provided 11 non-null splitting measurements. The more northerly transition to the apparent isotropy may suggest that mantle delamination either occurs further north or that we are measuring anisotropy dominantly within the slab. We suggest that a deployment in a similar configuration/orientation to RATTIL, but which extends further north and intersects the WCNIPSE line may help to better delineate the transition point to the observed isotropy in the north.

Average delay times show large variations at individual stations and for measure- 
ments from the same earthquake at different stations (Figure 5.10 and 5.11). Generally, observed delay times are higher for stations located closest to the TRL and for those located north of the TRL (Figure 5.12). However, the large errors in delay times make interpretation difficult. We observe generally larger mean delay times on individual stations that are located north of the TRL ( $2.1 \mathrm{~s} \pm 0.25 \mathrm{~s})$, compared to south of the TRL $(1.8 \mathrm{~s} \pm 0.12 \mathrm{~s})$. This may suggest that the thicker mantle may contribute to the observed larger delay times in the north. With a shear wave velocity of $1.4 \%$ found in the subslab mantle beneath an array by Gledhill and Stuart (1996), our station average delay time of $1.9 \mathrm{~s} \pm 0.11$ s suggests that anisotropy extends down to a depth of at least $500 \mathrm{~km}$. Because only teleseismic phases were examined in this study we are unable to constrain the vertical extent of anisotropy as we can not account for varying anisotropy with depth.

\subsection{Conclusions}

There have been limited in-depth seismic anisotropy studies between Taranaki and Ruapehu volcanoes, the location of a 'step' in the Moho marked by the TRL. Our study acts to fill in SWS measurements in this region and investigates the influence of this geophysical phenomenon on our seismic anisotropy measurements. Teleseismic XKS events recorded at 10 broadband seismometers on the RATTIL array have been used to investigate variations in anisotropy across the TRL.

We have found that:

1. Most teleseismic SWS studies suggest to use earthquakes with a lower magnitude limit of Mw 6, however our measurements suggest that a lower limit of Mw 5 in this region is appropriate.

2. Splitting parameters are remarkably consistent across the TRL with dominant NE-SW, trench-parallel, fast azimuths $\left(43^{\circ} \pm 5^{\circ}\right)$. The lack of variations in fast azimuths suggests minor, if any, influence from the observed step in the Moho or change in the lithosphere on SWS fast azimuths.

3. The similarity of our observed fast azimuths to stations located east of the mantle wedge in previous studies in eastern and southern North Island, suggests that similar fast azimuths are observed in the mantle wedge and subslab mantle. 
4. We interpret our NE-SW fast azimuths are due to a dominant shear deformation, associated with oblique convergence and that flow is in a trench-parallel direction.

5. Delay times increase for stations located closest to the TRL may suggest some influence from the step in the Moho; however, large errors make interpretation difficult.

6. Teleseismic SWS results suggest that the transition to the apparent isotropy observed in previous studies in this region is located north of the RATTIL array (north of approximately $-38.75^{\circ}$ ). The more northerly transition to the apparent isotropy may suggest that mantle delamination either occurs further north or that we are measuring anisotropy dominantly within the slab.

We suggest that future work should include the SWS of local events using the RATTIL stations for a direct comparison of upper mantle and crustal splitting measurements on the same stations. A study of crustal anisotropy may help reveal changes in response to the TRL boundary. 


\section{Chapter 6}

\section{Conclusions}

The Hikurangi subduction zone in North Island, New Zealand, is an example of a deforming plate boundary where stress build-up is released through both seismic and aseismic (i.e., slow) slip events. Better understanding of the underlying processes is of great importance for public safety planning in this region and in other subduction zones elsewhere. The abundance of seismic data for North Island provides an excellent opportunity to characterize the underlying stress and strain, and to improve on the existing methodologies of geophysical investigation. In particular, shear wave splitting (SWS) allows us to examine the seismic anisotropy beneath the surface of the Earth and is one of the few methods available to measure stress in the crust and lithosphere as well as strain and flow in the mantle. In this thesis, we provide an extensive analysis of crustal and upper mantle seismic anisotropy, via SWS, across the Hikurangi subduction zone of the North Island. For a comprehensive insight, we focused on three geographical regions across the Hikurangi subduction zone for which distinct seismic datasets are available from the HOBITSS and RATTIL experiments and the GeoNet network. Our results shed light on how anisotropy varies laterally across North Island, the underlying sources of anisotropy, and the temporal and spatial dynamics of slow slip as well as its mechanics. In this chapter we will answer questions posed at the start of this thesis:

1. A) What SWS parameters are observed at the shallowest portion of the subducting thrust, offshore the East Coast of the northern Hikurangi subduction zone? B) How does crustal anisotropy vary on-land across North Island? C) 
How do SWS parameters vary offshore, as opposed to on-land? D) How can we differentiate between areas of stress-induced and structurally-controlled anisotropy in the crust?

A) We studied an offshore region located off the East Coast of the northern Hikurangi subduction zone (Chapter 3). We calculated shear wave splitting using the automated method of Savage et al. (2010b) and measured $V_{P} / V_{S}$ ratios for eventstation pairs on HOBITSS ocean bottom seismometers and onshore GeoNet seismic stations. Spatial averaging of SWS fast azimuths using the method of Johnson et al. (2011) yielded trench-perpendicular fast azimuths in much of the offshore area, parallel to the relative plate convergence directions, suggesting that compressive stress from plate convergence closes microcracks and controls anisotropy in the upper-plate. Variations in the SWS fast azimuths from the trench perpendicular directions were observed radiating around a subducting seamount, with directions closely mimicking fault and fracture patterns created by subducting seamounts previously observed in both laboratory and field experiments. The resemblance of the fast azimuths to seamount fracture patterns suggests a structural control through faulting. However, temporal variations in fast azimuths at stations located above the seamount were also observed, suggesting a combination of stress and structural control on anisotropy. Temporal variations are commented on further in question 2. Thus, we find that offshore the East Coast of North Island, where the subducting plate is shallow, fast azimuths are dominantly trenchparallel and are most likely controlled by compressive stress from plate convergence. However, fast azimuths in an area of a shallow subducting seamount show a pattern which mimic fault and fracture patterns above subducted seamounts, suggesting areas of structural control can be observed offshore in regions where shallow pervasive structures exist.

B) In Chapter 4, we focused on the central North Island, which is covered by an array of permanent GeoNet stations. We measured SWS parameters using 20,835 local earthquakes recorded by 24 stations of the GeoNet Network (2009-2012). Using the recorded SWS measurements, over the 4 year time frame, we generated one of the largest datasets of crustal anisotropy measurements in central North Island comprising of 42,423 high-quality SWS measurements. We used the method of Johnson et al. (2011) to spatially average the fast azimuth measurements and compare them to stress orientations derived from continuous campaign GPS and 
gravitational stress calculations, as well as orientations of active faults. Spatial averaging of fast azimuths showed a dominant NE-SW orientation along the North Island Dextral Fault Belt (NIDFB). Fault orientations showed the closest agreement to our fast azimuths, suggesting a pervasive structural control. However, two regions, located in areas of thick sediment, show clear deviations from the structural trend and are more similar to the regional maximum compressional stress $S_{H} \max$. These include fast azimuths within the Wanganui Basin, as well as a small area along the NIDFB. We suggest that the thick sediments in these regions act to weaken the crustal fabric and our fast azimuths more closely represent the stress field at greater depths where $S_{H} \max$ directions are more closely aligned with the apparent plate motion. Fast azimuths around Mt. Ruapehu are in general agreement with both GPS-derived $S_{H} \max$ as well as local fault orientations. Previous studies have suggested both stress-induced and structural controls in the vicinity of the volcano and our results support these suggestions.

C) Contrary to the trench-perpendicular fast azimuths observed offshore, in central North Island, where the subducting plate is considerably deeper, fast azimuths are dominantly trench-parallel and are suggested to be structurally controlled by the pervasive fault fabric of the NIDFB. However, fast azimuths in regions of thick sedimentary basins exhibit directions closer to the regional maximum compressional stress $S_{H} \max$. This suggests that the thick sediments in these regions act to weaken the crustal fabric and our fast azimuths more closely represent the stress field at greater depths where $S_{H} \max$ directions are more closely aligned with the apparent plate motion. The spatial averaging of delay times reveals a significant increase towards the Hikurangi trench, and suggests high-fluid content and pervasive fractures in the top few kilometers of the crust.

D) Through these studies we have shown that with knowledge of the local and regional stress field, as well as structural elements in a region, it is possible to determine whether anisotropy is controlled by stress, structure, or a combination of both mechanisms. In order to determine areas of stress-induced versus structurally-controlled anisotropy, we have compared our SWS azimuths to several geophysical results, such as apparent plate convergence directions, seamount fracture and fault patterns, active fault orientations, Andersonian model of stresses (Anderson, 1905), Coulomb failure stress changes, stress ratios inferred from focal mechanism analysis, gravitational stresses $S_{H \max }^{\text {grav }}$, stress field from the inversion 
of focal mechanisms, and GPS-derived maximum compression directions. Using those comparative analyses, we successfully distinguished between the areas of stress-induced versus structurally-controlled anisotropy.

2. Does anisotropy vary temporally during slow slip? What are the mechanics causing these variations?

We examined temporal changes during a five-week long slow slip event (SSE) in September 2014 which occurred near Gisborne in the offshore region of the East Coast of the northern Hikurangi subduction zone, a region of frequent slow slip (Chapter 3). This SSE was recorded by offshore ocean-bottom seismometers (OBSs) deployed by the Hikurangi Ocean Bottom Investigation of Tremor and Slow Slip (HOBITSS) project. Temporal variations in $V_{P} / V_{S}$ and delay time measurements were observed on most OBSs during the September-October 2014 SSE, with a notable increase in $V_{P} / V_{S}$ and decrease in delay time occurring during the SSE. Stacking measurements across all offshore stations enhanced our signal, indicating that the changes occur over a broad region, and revealed clear opposing trends in delay time and $V_{P} / V_{S}$. We underline the importance of the use of these two parameters in conjunction, as the inverse variation of $V_{P} / V_{S}$ and delay time measurements allowed for the distinction between physical variations and variations in data. We interpreted this trend in $\delta t$ and $V_{P} / V_{S}$ as changes in differential horizontal stress that act to change crack aspect ratios and orientations. The increase in $V_{P} / V_{S}$ and decrease in delay time during the SSE suggests that an interconnection of cracks allows for fluid transfer upwards from below the previously sealed, possibly over-pressured subducting plate. These observations are consistent with fluctuations in retrieved stress ratios, which indicate changes in pore fluid pressure in the subducting crust, during multiple SSEs recorded by the HOBITSS experiment (Warren-Smith et al., 2019).

Temporal variations in fast azimuths were observed at HOBITSS stations located above a seamount, suggesting measurable variations in stress orientations. The upper-plate fracture network above the subducted seamount is the host of migrating fluids, from over-pressured sediments downdip of the seamount, resulting in a complicated environment of stress distribution (Shaddox and Schwartz, 2019). The changes in $\Phi$ at stations located near the subducting seamount were interpreted as rotations in microcracks corresponding to stress changes associated with 
the September-October 2014 SSE. The temporal variations in $\Phi$ at individual stations, along with the similarity of spatially averaged fast azimuths to structural patterns around the subducting seamount, suggest a combination of stress and structural effects due to the interactions of subducting seamounts with the overriding plate.

In Chapter 4, we searched for temporal variations during the 18-month long 20102011 Manawatu slow slip event; however, we did not observe significant temporal variations during the SSE. This was in part due to large confidence intervals resulting from insufficient measurements. The shallow nature and short duration of the September-October 2014 Gisborne SSE, along with the dense network of seismic stations, were some of the key factors that allowed us to observe temporal variations in SWS during this SSE. In contrast, the long-duration and deep nature of the 2010 Manawatu SSE, along with the sparse station coverage, may have influenced our lack of ability to observe temporal variations in SWS during this event.

Through these studies, we demonstrate that SWS and $V_{P} / V_{S}$ are effective tools for investigating stress changes associated with slow slip. Moreover, our results provide further evidence that fluids and stresses play a significant role in SSE nucleation.

3. Do SWS parameters from teleseismic phases change across the TaranakiRuapehu Line and do they vary with depth? If so, what are the sources controlling the observed changes?

We investigated the relationship between seismic anisotropy and mantle deformation across a deep boundary in the mantle, marked by the Taranaki-Ruapehu Line (TRL), in the back-arc region of western North Island (Chapter 5). The TRL marks the boundary between a deeper crust ( $\sim 32 \mathrm{~km}$ thick) to the south and a shallower crust ( $\sim 25 \mathrm{~km}$ thick) in the north, interpreted as an abrupt step in the Moho. We analyzed teleseismic earthquakes recorded on the Ruapehu And Taranaki Teleseismic Imaging Line (RATTIL) network between 2012-2014. Splitting parameters were remarkably consistent across the TRL with dominant NE-SW, tench-parallel, fast azimuths $\left(42.5^{\circ} \pm 5^{\circ}\right)$. The lack of variations in fast azimuths suggests minor, if any, influence from the observed step in the Moho on SWS fast azimuths. The similarity of our observed fast azimuths to stations located east of the mantle wedge 
in previous studies suggests that similar fast azimuths are observed in the mantle wedge and subslab mantle. Similar to previous studies in this region, we interpret that the NE-SW orientation of our fast azimuths is a result of dominant shear deformation associated with oblique convergence, and that the flow is oriented in a trench-parallel direction. Our SWS results suggest that the transition to the apparent isotropy observed west of the TVZ in previous studies in this region is located north of the RATTIL array (north of approximately $-38.75^{\circ}$ ).

\subsection{Implications for future research}

In light of the work presented here, we envisage several avenues meriting further investigation. Our results from offshore the East Coast of the northern Hikurangi subduction zone (Chapter 3) demonstrate that SWS and $V_{P} / V_{S}$ are effective tools for investigating stress changes associated with slow slip. We recommend that future SSE monitoring studies should consider similar network configurations to the HOBITSS network to study events of similar size. Alternatively, larger regional networks could help define the spatial extent of observed changes. In order to use SWS and $V_{P} / V_{S}$ to study SSEs which occur over significantly longer time periods, greater depths, and larger areas (Chapter 4) compared to the five-week long 2014 Gisborne SSE (Chapter 3), we suggest that station configurations need to be adapted appropriately. In particular, the central North Island region suffers from sparse GeoNet station coverage south of Mt. Ruapehu and across the Wanganui Basin, in the region of recurring Manawatu SSEs. Densifying these regions with a temporary array of seismometers may substantially improve our ability to observe temporal variations in SWS and $V_{P} / V_{S}$. Additionally, because the 2-D spatial averaging of delay times and fast azimuths are only a first order approximation for the structure of anisotropy, full 3-D tomography of SWS measurements would enable us to gain a greater resolution of the regions and depths of anisotropy. Finally, we think that, in addition to our teleseismic SWS analysis (Chapter 5), it would be worthwhile to better constrain the anisotropy across the TRL, and it's relationship to the step in the Moho, by studying crustal anisotropy based on local earthquakes. 
Appendix A

Appendix A: Chapter 2

This chapter provides appendix material for Chapter 2: Methods. 
Table A.1: Information for earthquakes used in P and Rayleigh wave analyses.

\begin{tabular}{lccccccc}
\hline Date & Lat $\left(^{\circ}\right)$ & Long $\left(^{\circ}\right)$ & Depth $(\mathrm{km})$ & $\mathrm{Mw}$ & BAZ $\left(^{\circ}\right)$ & Distance $(\mathrm{km})$ & Method $(\mathrm{P}-\mathrm{R})$ \\
\hline $12 / 05 / 2014$ & -49.94 & -114.80 & 11 & 6.5 & 126 & 5254 & $\mathrm{R}$ \\
$13 / 05 / 2014$ & 7.21 & -82.31 & 10 & 6.5 & 90 & 11290 & $\mathrm{R}$ \\
$14 / 05 / 2014$ & 6.45 & 144.92 & 10 & 6.1 & 317 & 6097 & $\mathrm{P}$ \\
$15 / 05 / 2014$ & 6.43 & 144.94 & 11 & 6.3 & 317 & 6094 & $\mathrm{P}$ \\
$23 / 06 / 2014$ & 51.85 & 178.74 & 109 & 7.9 & 0 & 10040 & $\mathrm{R}$ \\
$29 / 06 / 2014$ & 24.39 & 142.63 & 48 & 6.2 & 325 & 7949 & $\mathrm{P}-\mathrm{R}$ \\
$29 / 06 / 2014$ & -55.47 & -28.37 & 8 & 6.9 & 165 & 9233 & $\mathrm{P}-\mathrm{R}$ \\
$4 / 07 / 2014$ & -6.23 & 152.81 & 20 & 6.5 & 318 & 4441 & $\mathrm{P}$ \\
$5 / 07 / 2014$ & 1.93 & 96.94 & 20 & 6.0 & 277 & 9430 & $\mathrm{R}$ \\
$7 / 07 / 2014$ & 14.72 & -92.46 & 53 & 6.9 & 78 & 10904 & $\mathrm{R}$ \\
$3 / 08 / 2014$ & 82.95 & 146.17 & 13 & 6.9 & 315 & 5517 & $\mathrm{P}$ \\
$3 / 08 / 2014$ & 27.19 & 103.41 & 12 & 6.2 & 300 & 10698 & $\mathrm{R}$ \\
$23 / 08 / 2014$ & -32.70 & -71.44 & 32 & 6.4 & 127 & 9268 & $\mathrm{P}$ \\
$6 / 09 / 2014$ & -2.67 & -1.15 & 7 & 6.1 & 99 & 625 & $\mathrm{R}$ \\
$17 / 09 / 2014$ & 13.76 & 144.43 & 130 & 6.7 & 322 & 6816 & $\mathrm{P}$ \\
$25 / 09 / 2014$ & -9.46 & 156.41 & 4 & 6.1 & 320 & 3929 & $\mathrm{P}$ \\
$9 / 10 / 2014$ & -32.11 & -110.81 & 17 & 7.0 & 106 & 6266 & $\mathrm{P}-\mathrm{R}$ \\
$9 / 10 / 2014$ & -32.10 & -110.87 & 10 & 6.6 & 106 & 6263 & $\mathrm{R}$ \\
$14 / 10 / 2014$ & 12.53 & -88.12 & 40 & 7.3 & 82 & 11125 & $\mathrm{R}$ \\
$3 / 11 / 2014$ & -41.73 & 79.96 & 10 & 6.3 & 231 & 7886 & $\mathrm{R}$
\end{tabular}


Table A.1 continued from previous page

\begin{tabular}{lccccccc}
\hline Date & Lat $\left(^{\circ}\right)$ & Long $\left(^{\circ}\right)$ & Depth $(\mathrm{km})$ & $\mathrm{Mw}$ & BAZ $\left(^{\circ}\right)$ & Distance $(\mathrm{km})$ & Method (P-R) \\
\hline $7 / 11 / 2014$ & -5.99 & 148.23 & 53 & 6.6 & 312 & 4767 & $\mathrm{P}-\mathrm{R}$ \\
$15 / 11 / 2014$ & 1.89 & 126.52 & 45 & 7.1 & 297 & 6984 & $\mathrm{P}-\mathrm{R}$ \\
$21 / 11 / 2014$ & 2.30 & 127.05 & 35 & 6.5 & 298 & 6959 & $\mathrm{P}-\mathrm{R}$ \\
$22 / 11 / 2014$ & 36.64 & 137.89 & 9 & 6.2 & 328 & 9352 & $\mathrm{R}$ \\
$26 / 11 / 2014$ & 1.96 & 126.58 & 39 & 6.8 & 297 & 6985 & $\mathrm{P}$ \\
$2 / 11 / 2014$ & 6.16 & 123.13 & 61 & 6.6 & 298 & 7575 & $\mathrm{P}$ \\
$7 / 11 / 2014$ & -6.51 & 154.46 & 23 & 6.6 & 319 & 4342 & $\mathrm{P}$ \\
$7 / 11 / 2014$ & 13.67 & -91.47 & 32 & 6.1 & 79 & 10916 & $\mathrm{R}$ \\
$7 / 01 / 2015$ & 5.91 & -82.66 & 8 & 6.5 & 91 & 11146 & $\mathrm{R}$ \\
$13 / 02 / 2015$ & 22.64 & 121.43 & 30 & 6.2 & 308 & 9050 & $\mathrm{R}$ \\
$18 / 02 / 2015$ & -10.76 & 164.12 & 10 & 6.1 & 331 & 3421 & $\mathrm{P}$ \\
$22 / 02 / 2015$ & 18.68 & -106.85 & 5 & 6.2 & 66 & 10000 & $\mathrm{R}$ \\
$27 / 02 / 2015$ & -7.30 & 122.53 & 552 & 7.0 & 287 & 6588 & $\mathrm{P}$ \\
$18 / 03 / 2015$ & -36.12 & -73.52 & 13 & 6.2 & 128 & 8873 & $\mathrm{R}$ \\
$29 / 03 / 2015$ & -4.73 & 152.56 & 41 & 7.5 & 318 & 4619 & $\mathrm{P}$ \\
$31 / 03 / 2015$ & -4.89 & 152.49 & 39 & 6.0 & 318 & 4585 & $\mathrm{P}$ \\
$20 / 04 / 2015$ & 24.20 & 122.32 & 29 & 6.4 & 310 & 9120 & $\mathrm{P}$ \\
$24 / 04 / 2015$ & 51.62 & -130.77 & 8 & 6.2 & 29 & 11152 & $\mathrm{R}$ \\
$25 / 04 / 2015$ & 28.23 & 84.73 & 8 & 7.8 & 290 & 12231 & $\mathrm{R}$ \\
$25 / 04 / 2015$ & 28.22 & 84.82 & 10 & 6.6 & 290 & 12224 & $\mathrm{R}$
\end{tabular}


Table A.1 continued from previous page

\begin{tabular}{lccccccc}
\hline Date & Lat $\left(^{\circ}\right)$ & Long $\left(^{\circ}\right)$ & Depth $(\mathrm{km})$ & Mw & BAZ $\left(^{\circ}\right)$ & Distance $(\mathrm{km})$ & Method $(\mathrm{P}-\mathrm{R})$ \\
\hline $30 / 04 / 2015$ & -5.38 & 151.77 & 31 & 6.7 & 317 & 4604 & $\mathrm{P}$ \\
$1 / 05 / 2015$ & -5.20 & 151.78 & 44 & 6.8 & 317 & 4620 & $\mathrm{R}$ \\
$3 / 05 / 2015$ & -5.63 & 151.68 & 24 & 6.0 & 317 & 4586 & $\mathrm{R}$ \\
$5 / 05 / 2015$ & -5.46 & 151.88 & 55 & 7.5 & 317 & 4590 & $\mathrm{P}-\mathrm{R}$ \\
$7 / 05 / 2015$ & -7.22 & 154.56 & 10 & 7.1 & 319 & 4270 & $\mathrm{P}$ \\
$10 / 05 / 2015$ & 31.24 & 142.02 & 6 & 6.0 & 328 & 8643 & $\mathrm{R}$ \\
$12 / 05 / 2015$ & 38.91 & 142.03 & 35 & 6.8 & 332 & 9391 & $\mathrm{P}$ \\
$12 / 05 / 2015$ & 27.63 & 86.16 & 15 & 6.3 & 291 & 12077 & $\mathrm{R}$ \\
$20 / 05 / 2015$ & -10.88 & 164.17 & 11 & 6.8 & 331 & 3431 & $\mathrm{P}$ \\
$22 / 05 / 2015$ & -11.06 & 163.70 & 11 & 6.9 & 330 & 3432 & $\mathrm{P}$ \\
$23 / 05 / 2015$ & -11.11 & 163.22 & 10 & 6.8 & 329 & 3448 & $\mathrm{P}$ \\
$24 / 05 / 2015$ & -16.86 & -14.17 & 10 & 6.3 & 165 & 13682 & $\mathrm{R}$ \\
$30 / 05 / 2015$ & 27.84 & 140.49 & 664 & 7.8 & 325 & 8386 & $\mathrm{P}$ \\
\hline
\end{tabular}

Note: Station LOBS6 was used to calculate distance and back azimuth owing to its central location within the array. 


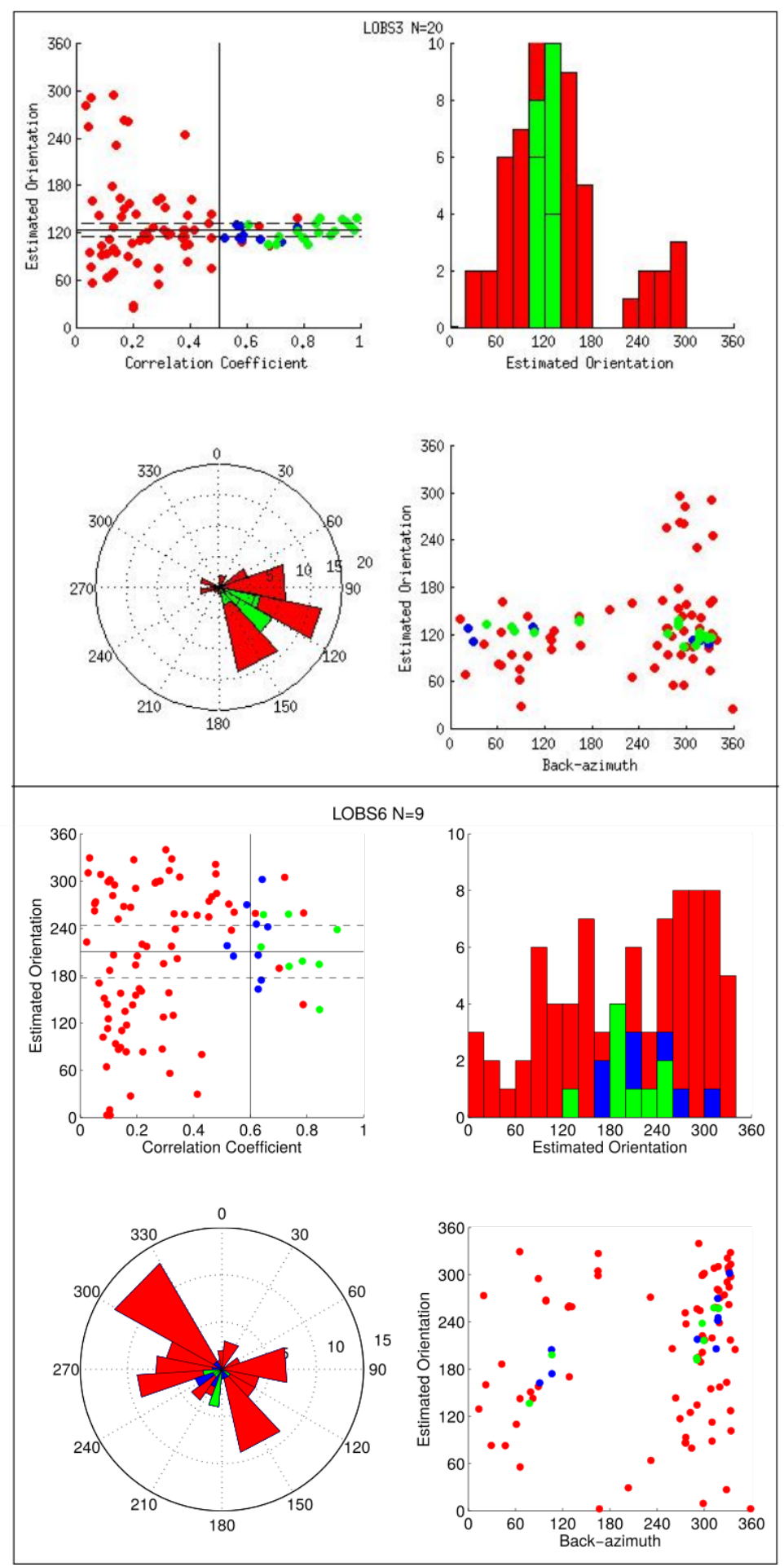

Figure A.1: Multi-part figure. Orientation estimates using Rayleigh-wave analysis for all HOBITSS stations used in Chapter 3. Subplots show: (top-left) correlation coefficient vs. estimated orientation with mean value and uncertainty range. Green dots are "good", blue are "questionable", and red are "bad" estimates. (top-right) histogram of estimated orientation, (bottom-left) polar histogram of estimated orientations, (bottom-right) event back azimuth versus estimated orientation. Only green, "Good", events are used to determine the average orientation value. 


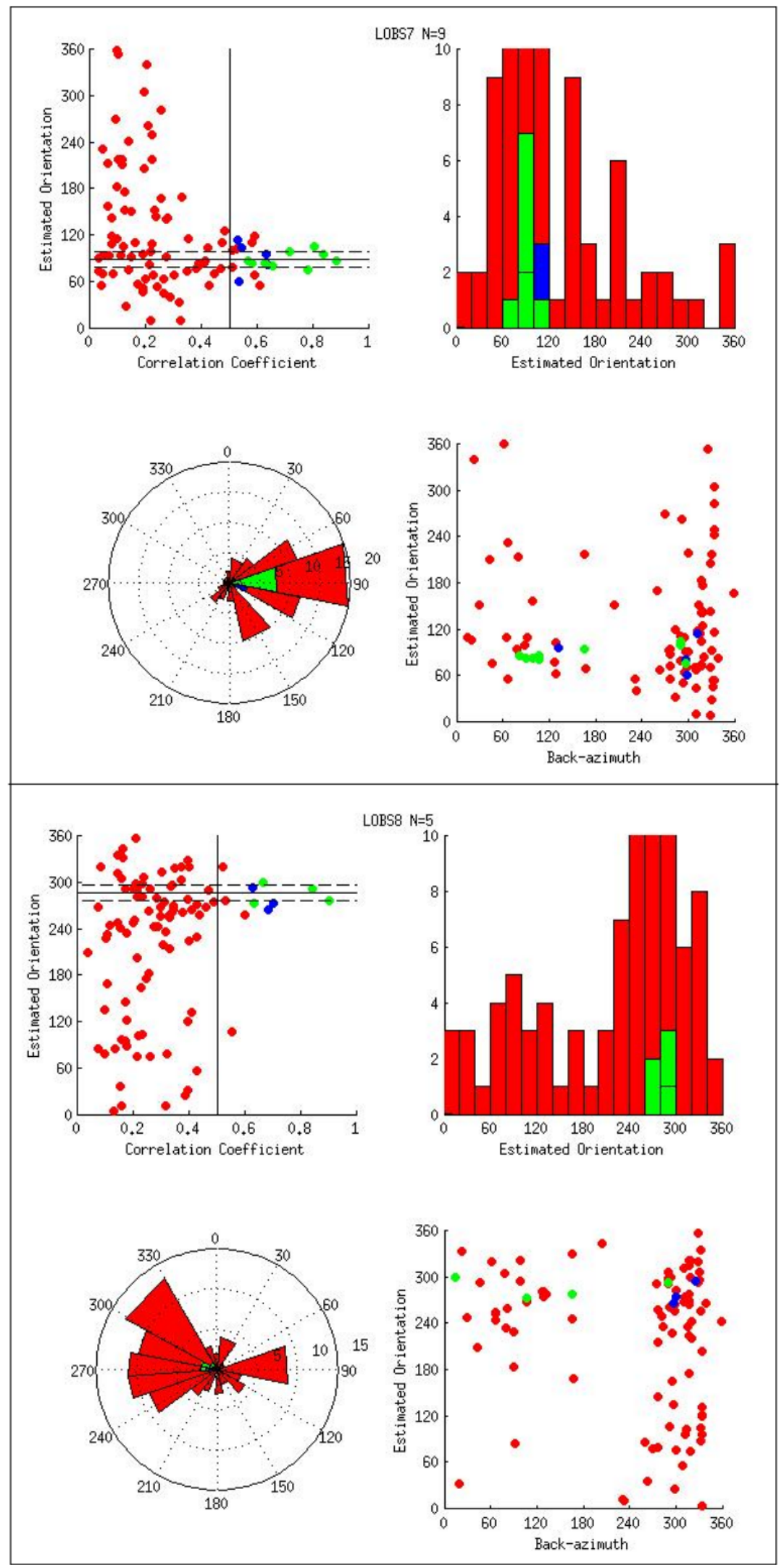

Figure A.1: (B) continued. 


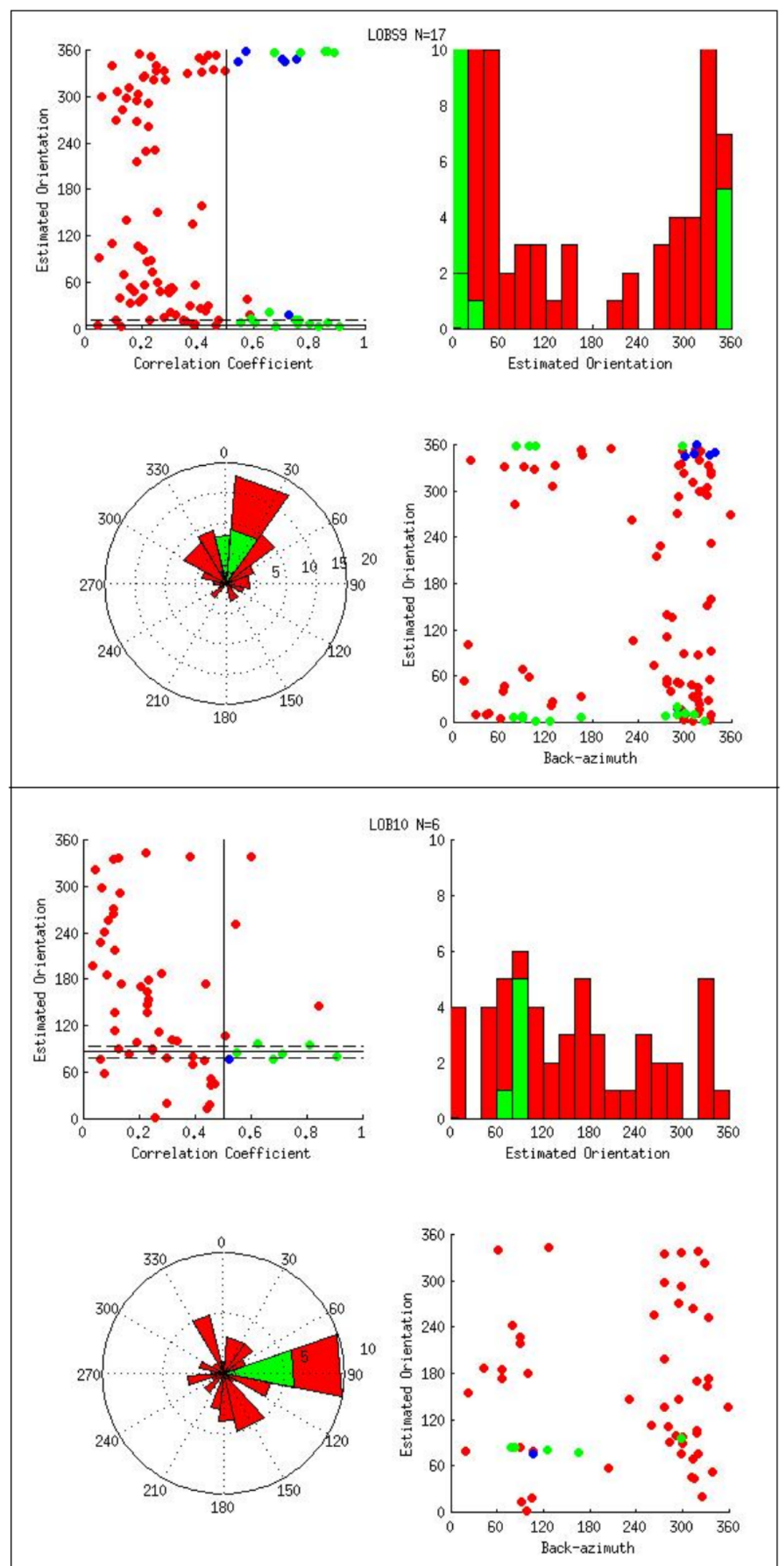

Figure A.1: (C) continued. 


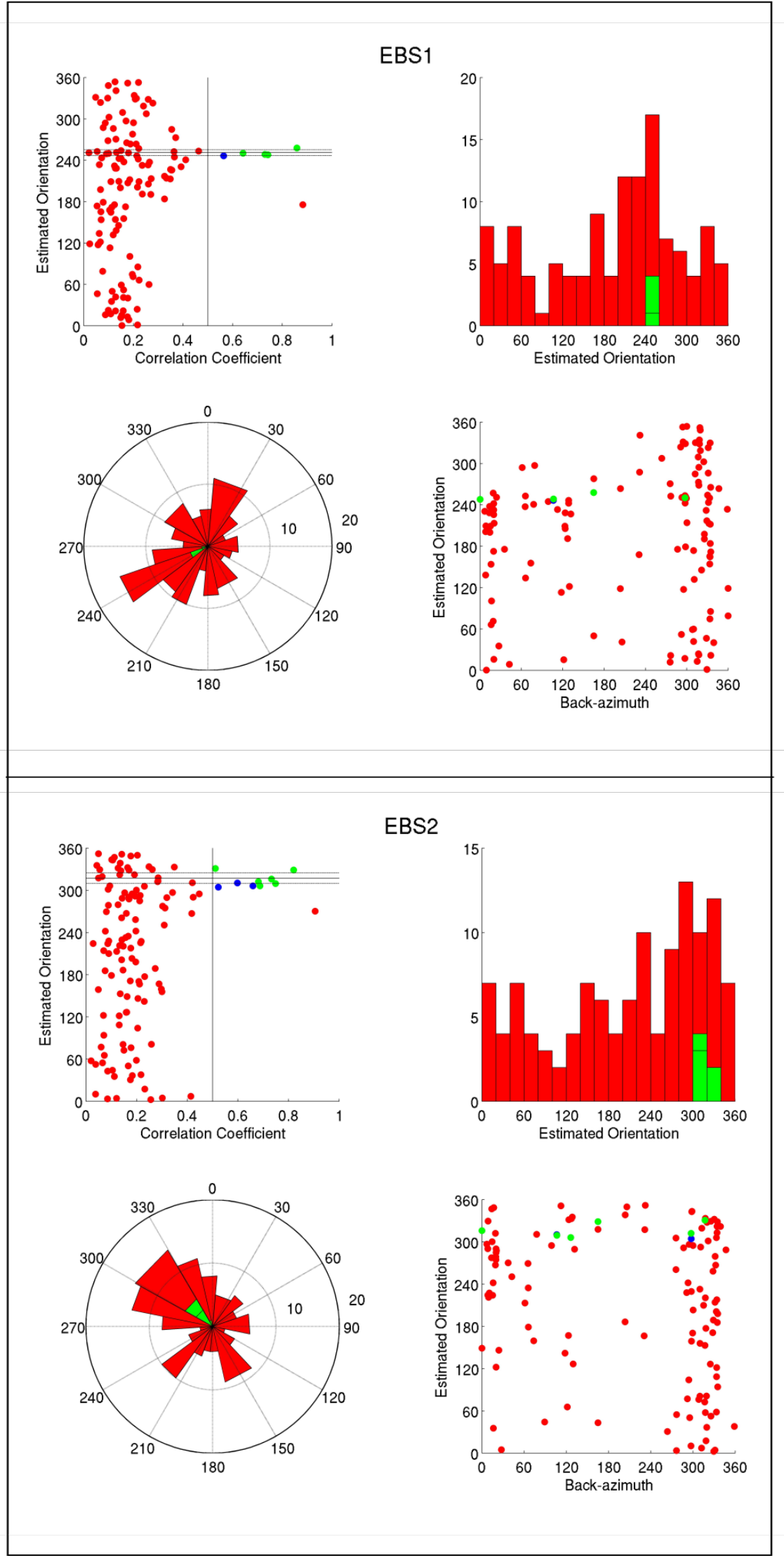

Figure A.1: (D) continued. 


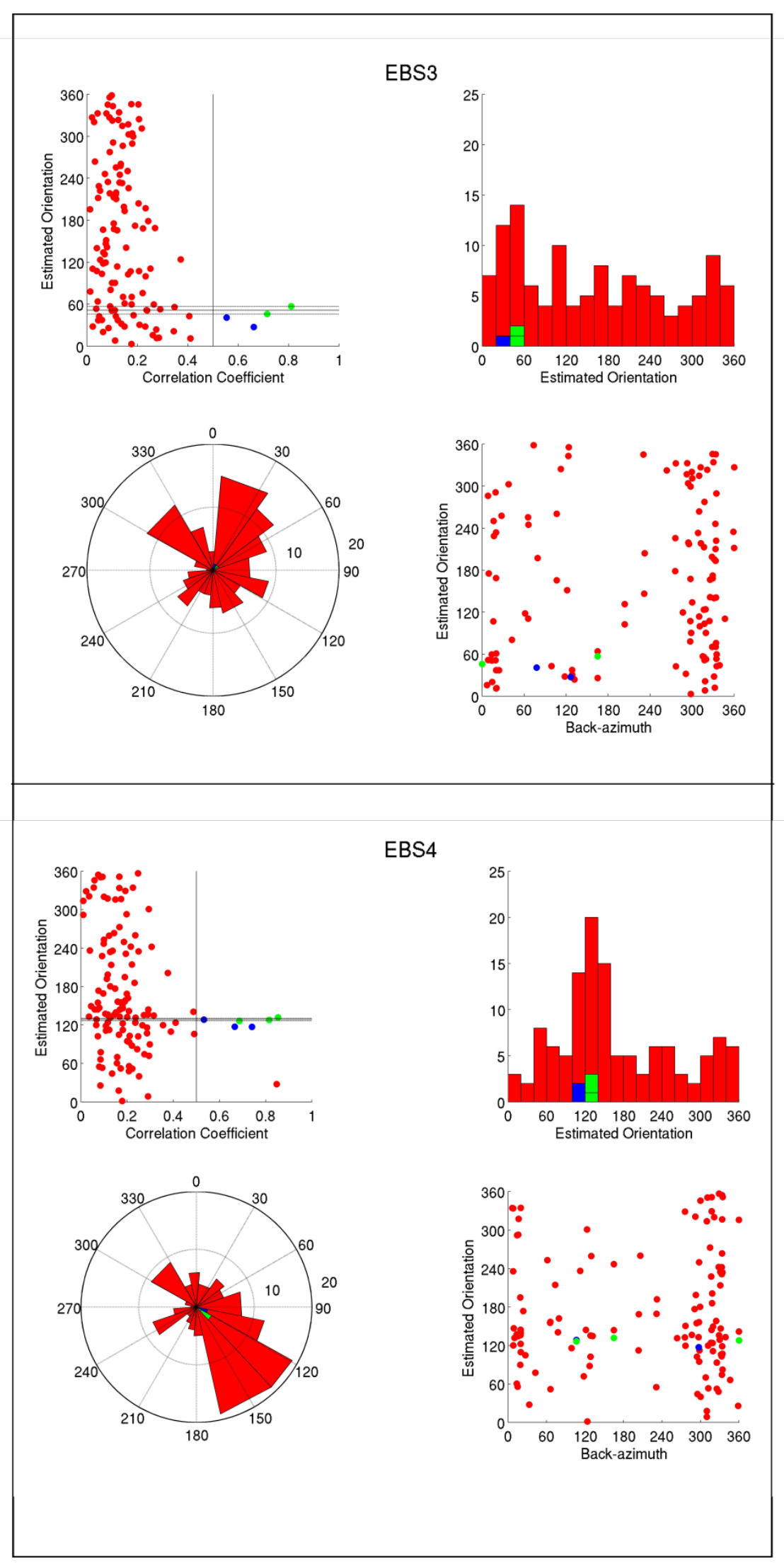

Figure A.1: (E) continued. 


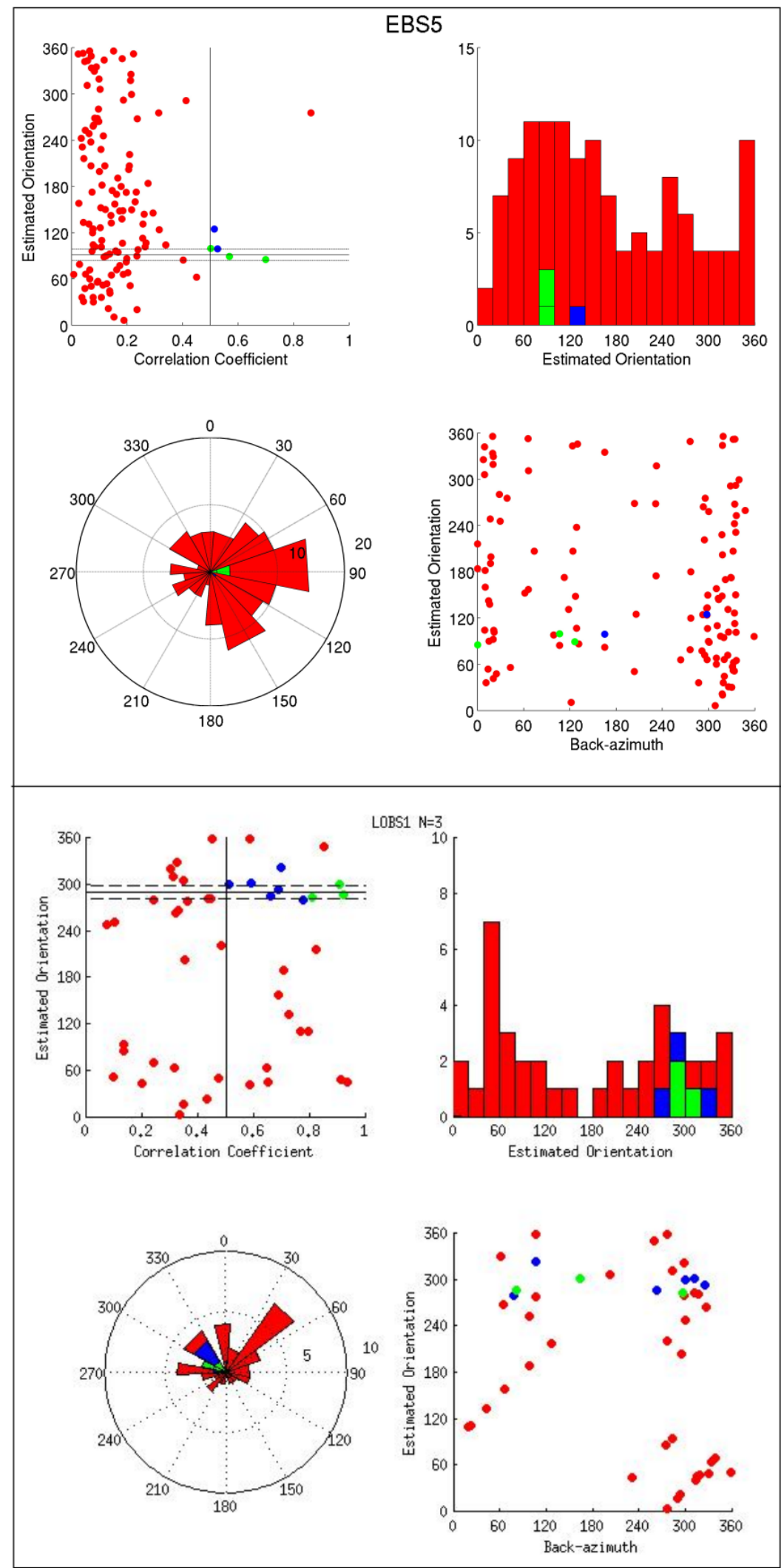

Figure A.1: (F) continued. 


\section{Appendix B}

\section{Appendix B: Chapter 3}

This chapter provides appendix material for Chapter 3: Temporal and spatial variations in seismic anisotropy and $V_{P} / V_{S}$ ratios in a region of slow slip.

\section{B.1 OBS timing and station corrections}

Stations LOBS3, 7 and 9 experienced timing issues. These timing issues do not affect SWS results but could alter $V_{P} / V_{S}$ results. To test for systematic affects from these stations we compared the average $V_{P} / V_{S}$ time variations for stations with and without timing errors (Figure B.1). We find minor differences in these two subsets, so these stations are included in the analyses. 
Table B.1: Velocity model used in MFAST for HOBITSS region.

\begin{tabular}{lccc}
\hline Depth & Vp & Vs & Density \\
\hline 0 & 4.72 & 2.62 & 2.56 \\
3 & 5.22 & 2.92 & 2.63 \\
8 & 5.66 & 3.18 & 2.68 \\
15 & 5.96 & 3.40 & 2.74 \\
23 & 6.36 & 3.58 & 2.85 \\
30 & 6.87 & 3.85 & 3.02 \\
38 & 7.74 & 4.36 & 3.27 \\
48 & 8.31 & 4.71 & 3.42 \\
65 & 8.89 & 5.07 & 3.56 \\
85 & 8.61 & 4.94 & 3.48 \\
105 & 8.44 & 4.83 & 3.45 \\
130 & 8.39 & 4.79 & 3.43 \\
155 & 8.38 & 4.79 & 3.43 \\
185 & 8.43 & 4.82 & 3.44 \\
225 & 8.56 & 4.89 & 3.47 \\
275 & 8.67 & 4.95 & 3.49 \\
370 & 8.96 & 5.18 & 3.57 \\
620 & 10.20 & 5.90 & 4.08 \\
750 & 10.60 & 6.13 & 4.23 \\
\hline
\end{tabular}



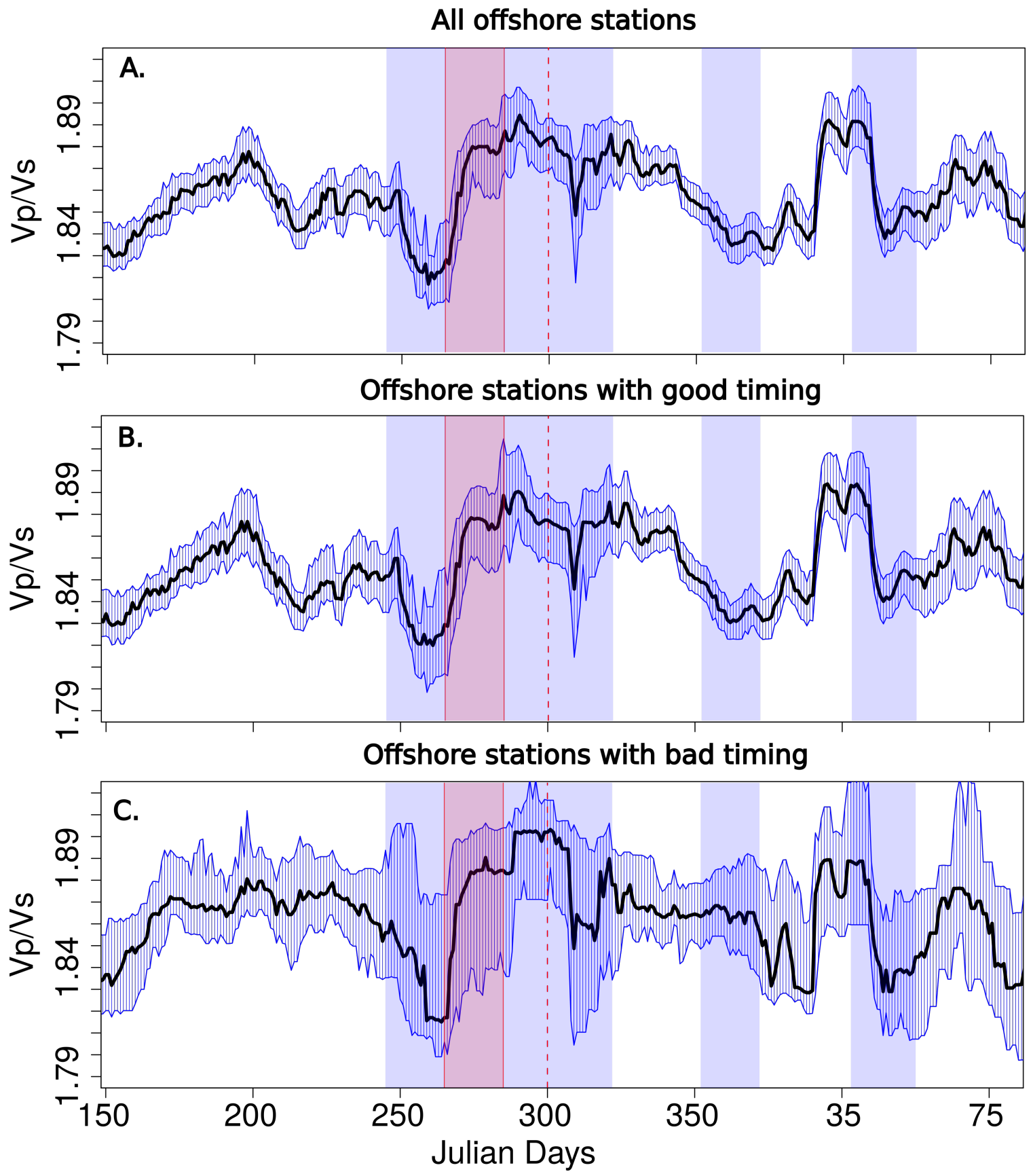

Figure B.1: A. 20 day moving median of $V_{P} / V_{S}$ for all offshore stations used in this study (LOBS3,7,8,9 and EOBS1-5). B. $V_{P} / V_{S}$ for stations with good timing (LOBS8 and EOBS1-5). C. $V_{P} / V_{S}$ using only stations with bad timing (LOBS3,7,9). The results from stations with bad timing (C) bear close resemblance to the full data set (A) and for this reason we choose to include the results from stations with bad timing. 


\section{B.2 Moving Averages and 95\% Confidence Intervals}

To look for temporal changes at each station, the moving median values of all $V_{P} / V_{S}$ and SWS delay time measurements were determined. To calculate the moving median of $n$ values $\left(d_{i}\right)$, where $i$ is between zero and $n$, the values were ranked and the median was represented by the middle value $\left(d_{\frac{n}{2}}\right)$. The $95 \%$ confidence limits of the median were calculated using the method of Altman (Altman et al., 2000). In this method, the lower $95 \%$ confidence limit is the ranked value $d_{l}$, where $l$ is given by:

$$
l=\left\lfloor\frac{n}{2}-\frac{1.96 \sqrt{n}}{2}\right\rceil
$$

and the upper $95 \%$ confidence limit is the ranked value $d_{u}$, where $u$ given by:

$$
u=\left\lfloor 1+\frac{n}{2}+\frac{1.96 \sqrt{n}}{2}\right\rceil
$$

Generally, the median is more robust than the mean, and less sensitive to outliers, but is more difficult to apply to azimuthal values (Altman et al., 2000). Therefore, values of fast polarization directions for the moving windows were calculated using a circular mean. These circular moving means were calculated using the 'circular' function (https:/ /CRAN.R-project.org/package=circular) in R. $95 \%$ confidence intervals of the mean were calculated by bootstrapping using the 'mle.vonmises.bootstrap.ci' function (https: / /CRAN.R-project.org/package=circular) in $\mathrm{R}$ and re-sampling the dataset 9999 times.

\section{B.3 Generation of Comparative Earthquake Sub-Catalogs}

When investigating temporal changes in SWS measurements, care must be taken to verify that any observed changes are not caused by changes in the spatial distribution of earthquakes. To accomplish this task, we found the closest earthquakes to each of the 159 earthquakes occurring during SSE2 in the catalog both before and after the SSE. In order to evaluate the sub-catalog similarities, we calculated the distance between each earthquake in all three sub-catalogs to a central point, station $\mathrm{CNGZ}$, and then compared the distribution of distances using a two-sample Kolmogorov-Smirnov Goodness-of-Fit test. The K-S tests returned a p-value of 0.99 for both before to during and during to after comparisons indicating all sets could reasonably be from the same distribution. This also indicates that 
the sub-catalogs are sufficiently similar to be used to test for temporal deviation in $V_{P} / V_{S}$ and delay time. Supplementary Figure B.5 shows the earthquakes from the three periods in map view as well as distance and azimuthal distributions.

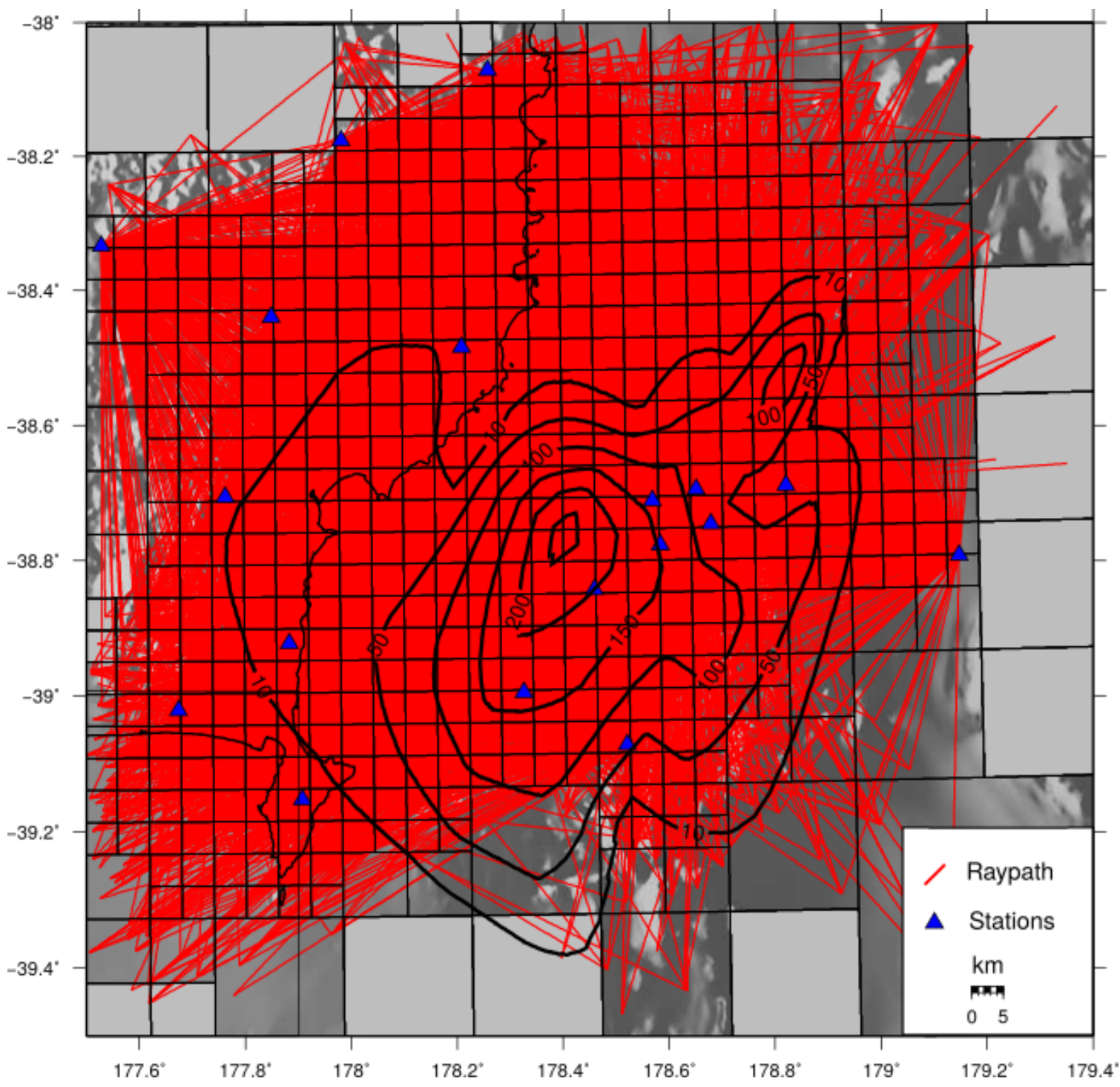

Figure B.2: Raypath plot created using quadtree gridding following the method described by Johnson et al. (2011). Triangles mark locations of seismic stations, and black lines show raypaths. A minimum box size of $3 \mathrm{~km}$ is chosen, containing between 10 and 65 raypaths passing through each box. Gray shaded boxes did not contain sufficient raypath coverage and were not used. Slip contours for SSE2 are from Wallace et al. (2016). 

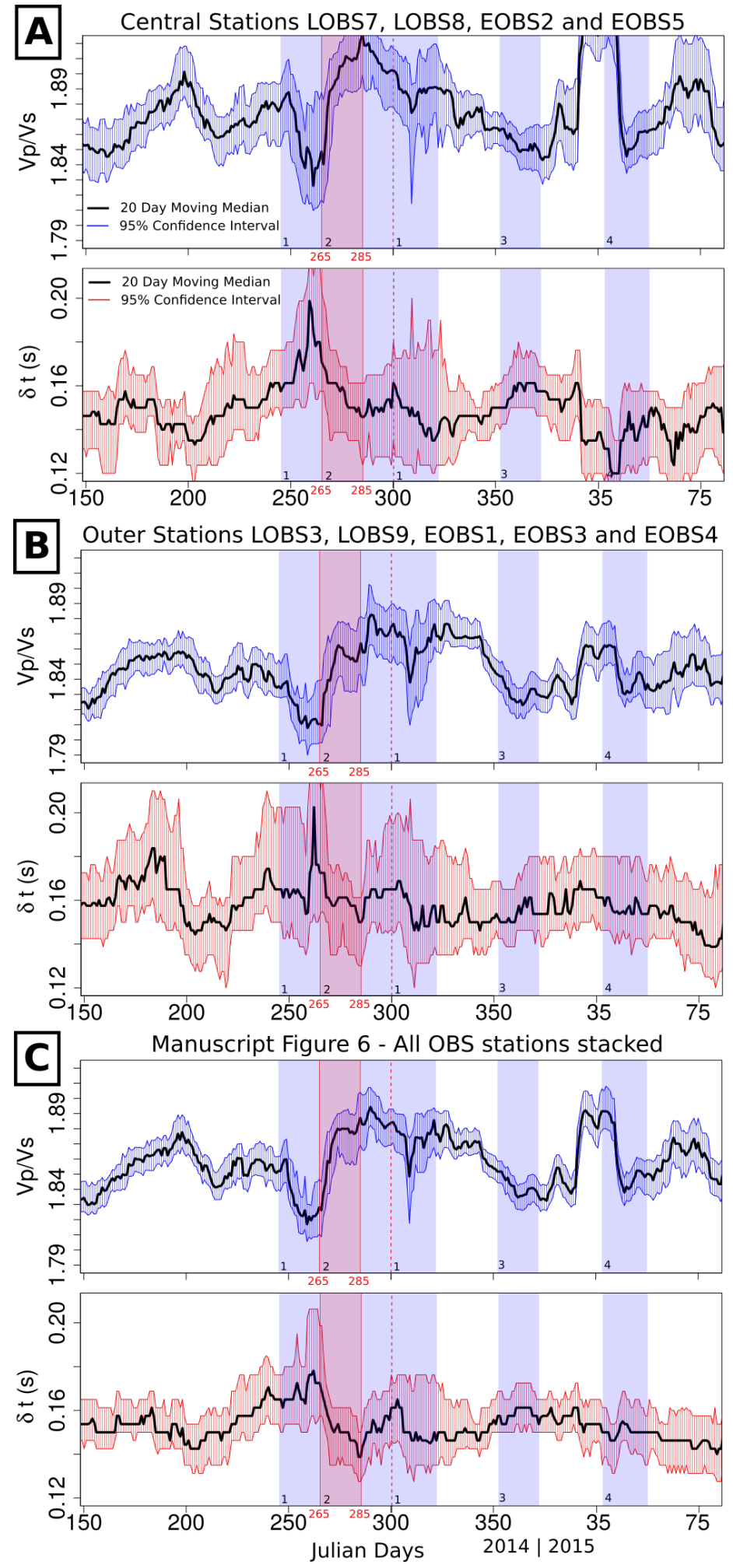

Figure B.3: A: Temporal plots of $V_{P} / V_{S}$ and delay time by stacking measurements using only the 4 most centrally located offshore stations (LOBS7, LOBS8, EOBS2 and EOBS5), with respect to SSE2. B: Temporal plots of $V_{P} / V_{S}$ and delay time by stacking measurements using only stations located outside of SSE2 (LOBS3, LOBS9, EOBS1, EOBS3, and EOBS4). C: Temporal plots of $V_{P} / V_{S}$ and delay time by stacking measurements from all offshore stations. 

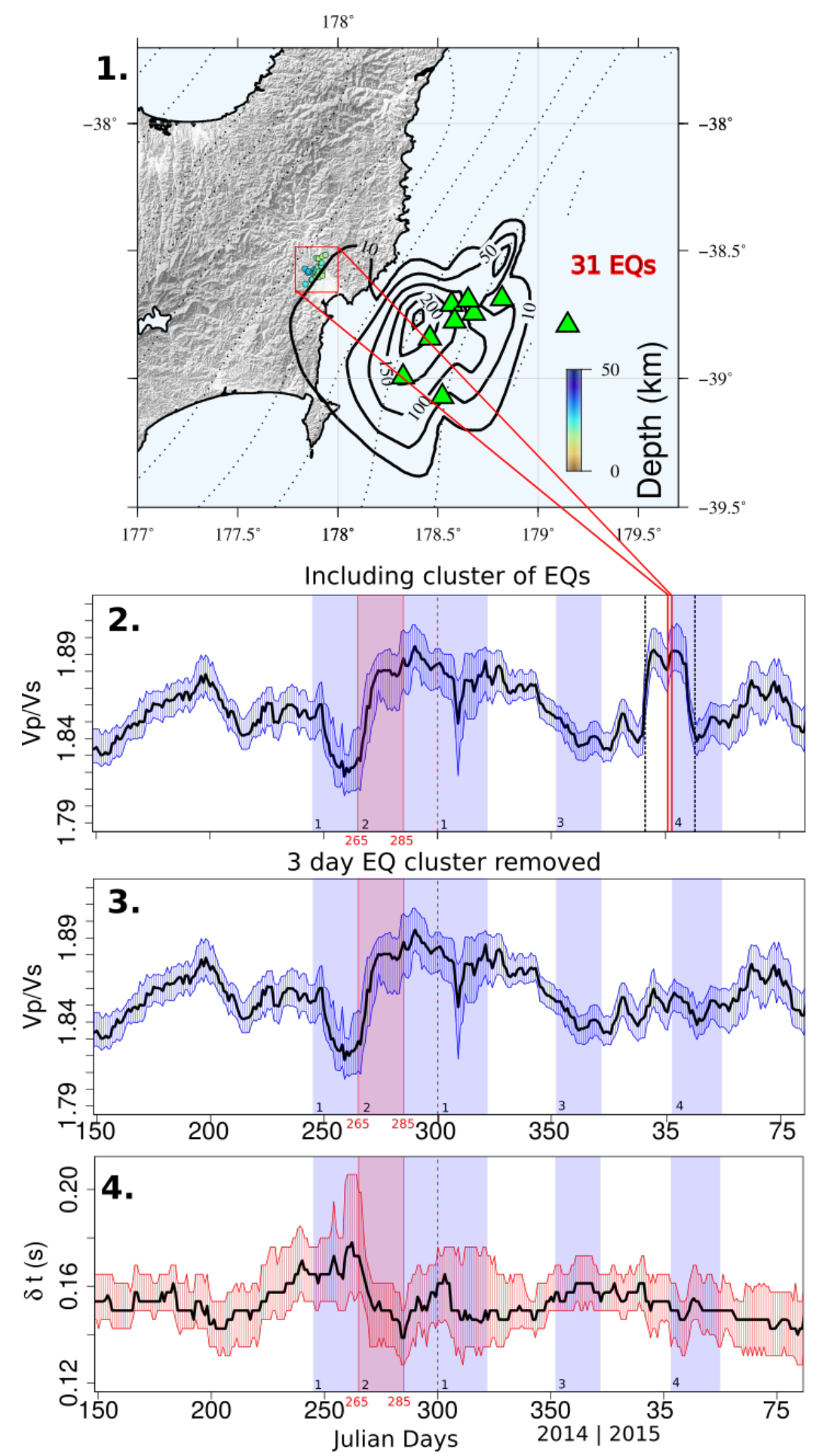

Figure B.4: 1: Plot of location of cluster of 31 earthquakes occurring between Julian days 36-38, out of 54 total earthquakes during this time. Green triangles show locations of offshore stations used in this study. 2: Plot of $V_{P} / V_{S}$ showing time of the 31 earthquakes, marked by red vertical lines. Black dashed vertical lines mark 10 days on either side of the cluster of earthquakes. A large increase in $V_{P} / V_{S}$ occurs at day 26 as a result of these 31 earthquakes. 3: Plot showing $V_{P} / V_{S}$ with the 3 day cluster of 31 earthquakes removed. 4: The delay time plot does not show any variation around Julian day 36. 

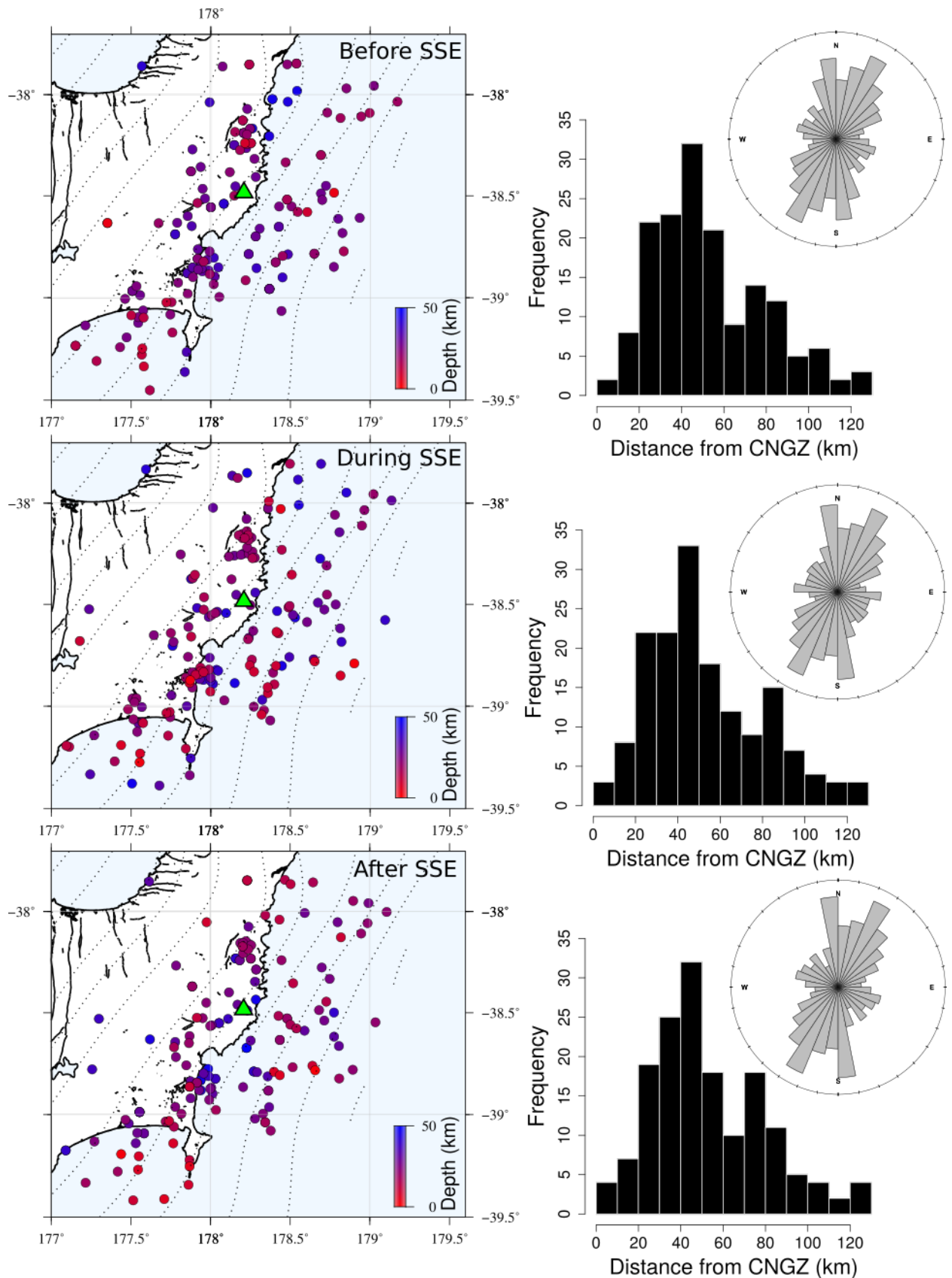

Figure B.5: Distribution of earthquakes in the spatial sub-catalogues based on events occurring during SSE2, from Julian day 265-300. Plots show distributions of earthquakes before, during, and after SSE2 with histograms of distributions of earthquake distances from GeoNet station CNGZ (green triangle), as well as rose plots showing variation in earthquake azimuths from station CNGZ. 

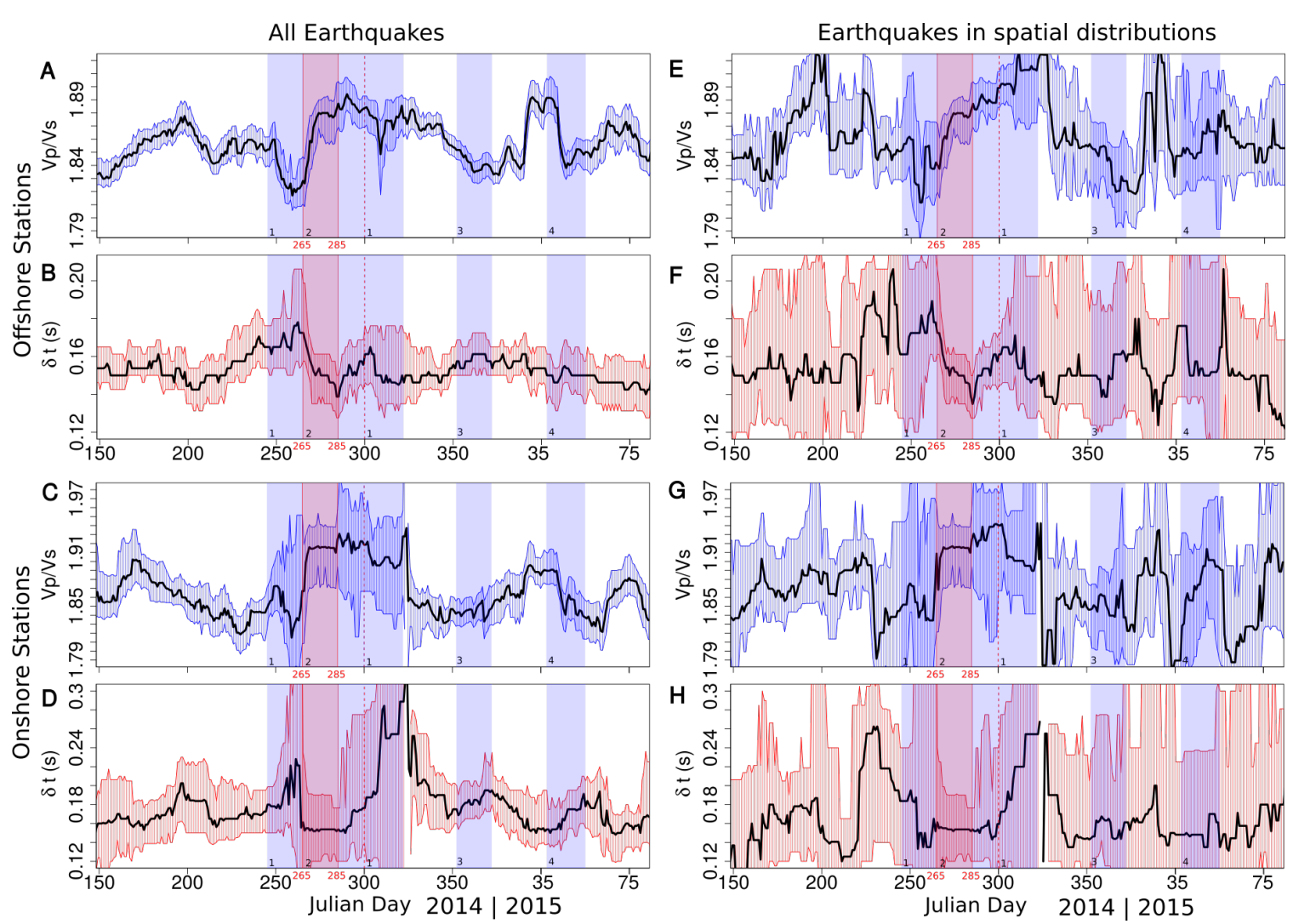

Figure B.6: (A) $V_{P} / V_{S}$ change on stack of all offshore stations for entire catalog $(9,056$ measurements) with upper and lower $95 \%$ confidence intervals. (B) Delay time change on all offshore station stack for entire catalog (8,333 measurements) with upper and lower 95\% confidence intervals. (C) $V_{P} / V_{S}$ change stack of all onshore stations for entire catalog $(4,124$ measurements). (D) Delay time change on all onshore station stack for entire catalog (2,343 measurements). (E) $V_{P} / V_{S}$ change on stack of all offshore stations for earthquakes occurring in spatial sub-catalogs from Figure B.5. (F) Delay time change on stack on all offshore stations for earthquakes occurring in spatial sub-catalogs. (G) $V_{P} / V_{S}$ change on stack on all onshore stations for earthquakes in spatial sub-catalogs. (H) Delay time change on stack on all onshore stations for earthquakes in spatial sub-catalog. 


\section{Vp/Vs vs Angle of Incidence at Surface}

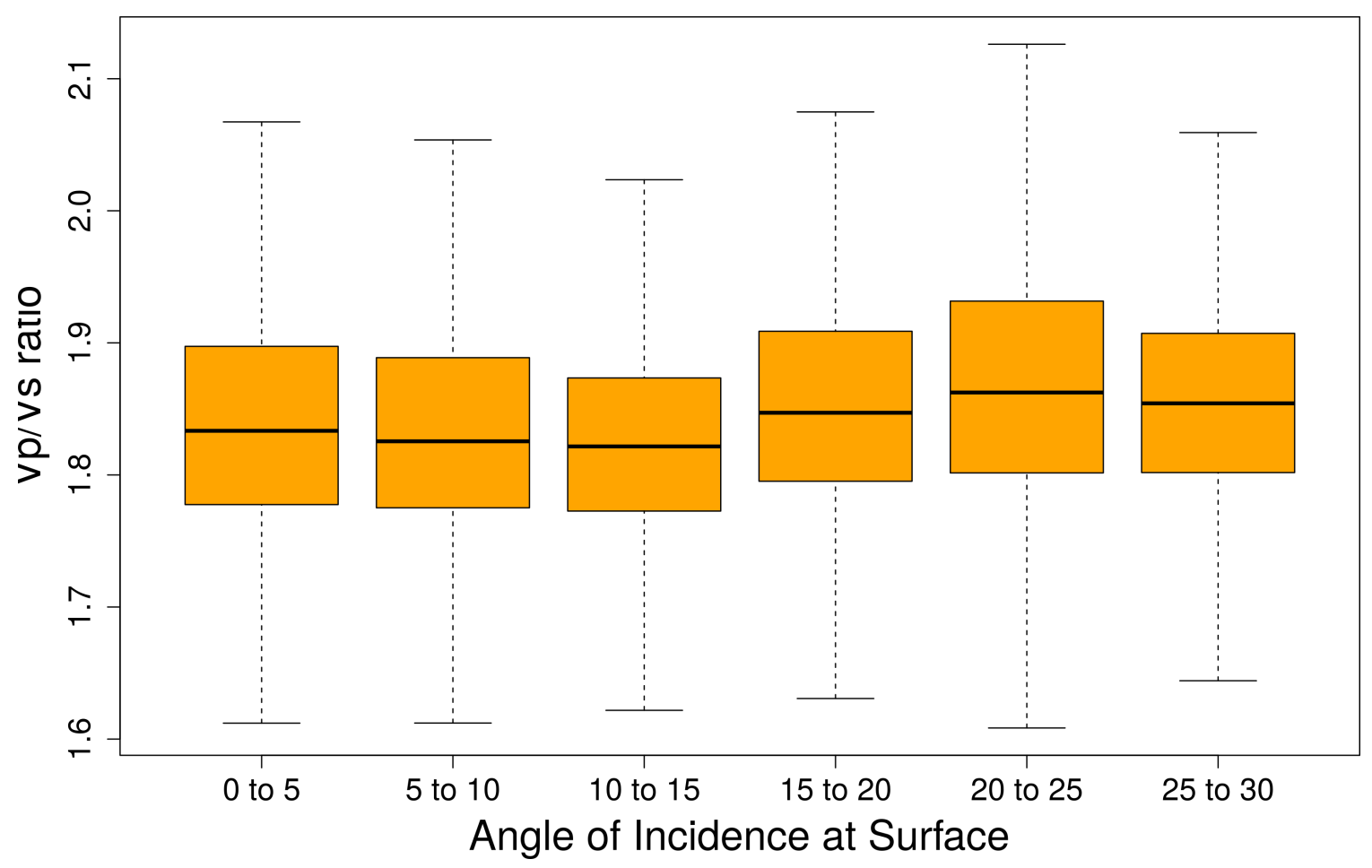

Figure B.7: Box and Whisker plot of angle of incidence versus corrected $V_{P} / V_{S}$ ratio for stack of offshore station measurements. Horizontal line shows median of each 5 degree interval. Whiskers show largest and smallest values that are not considered as outliers. Lower and upper box sides show first and third quartiles, respectively. We do not observe significant variation in $V_{P} / V_{S}$ with changing angle of incidence. 


\section{Appendix C}

\section{Appendix C: Chapter 4}

This chapter provides appendix material for Chapter 4: Spatial variations in seismic anisotropy in central North Island, New Zealand. 
Table C.1: Velocity model used in MFAST for central North Island region.

\begin{tabular}{lccc}
\hline Depth & Vp & Vs & Density \\
\hline 0 & 3.00 & 1.70 & 2.62 \\
3 & 5.15 & 2.93 & 2.62 \\
8 & 5.71 & 3.28 & 2.70 \\
15 & 6.02 & 3.47 & 2.76 \\
23 & 6.39 & 3.66 & 2.86 \\
30 & 6.88 & 3.91 & 3.02 \\
38 & 7.62 & 4.31 & 3.24 \\
48 & 8.02 & 4.56 & 3.35 \\
65 & 8.34 & 4.73 & 3.42 \\
85 & 8.51 & 4.86 & 3.46 \\
105 & 8.55 & 4.90 & 3.47 \\
130 & 8.51 & 4.88 & 3.46 \\
155 & 8.46 & 4.84 & 3.45 \\
185 & 8.50 & 4.86 & 3.46 \\
225 & 8.59 & 4.91 & 3.48 \\
275 & 8.68 & 4.96 & 3.49 \\
370 & 8.96 & 5.17 & 3.56 \\
620 & 10.20 & 5.90 & 4.08 \\
750 & 10.60 & 6.13 & 4.23 \\
\hline
\end{tabular}



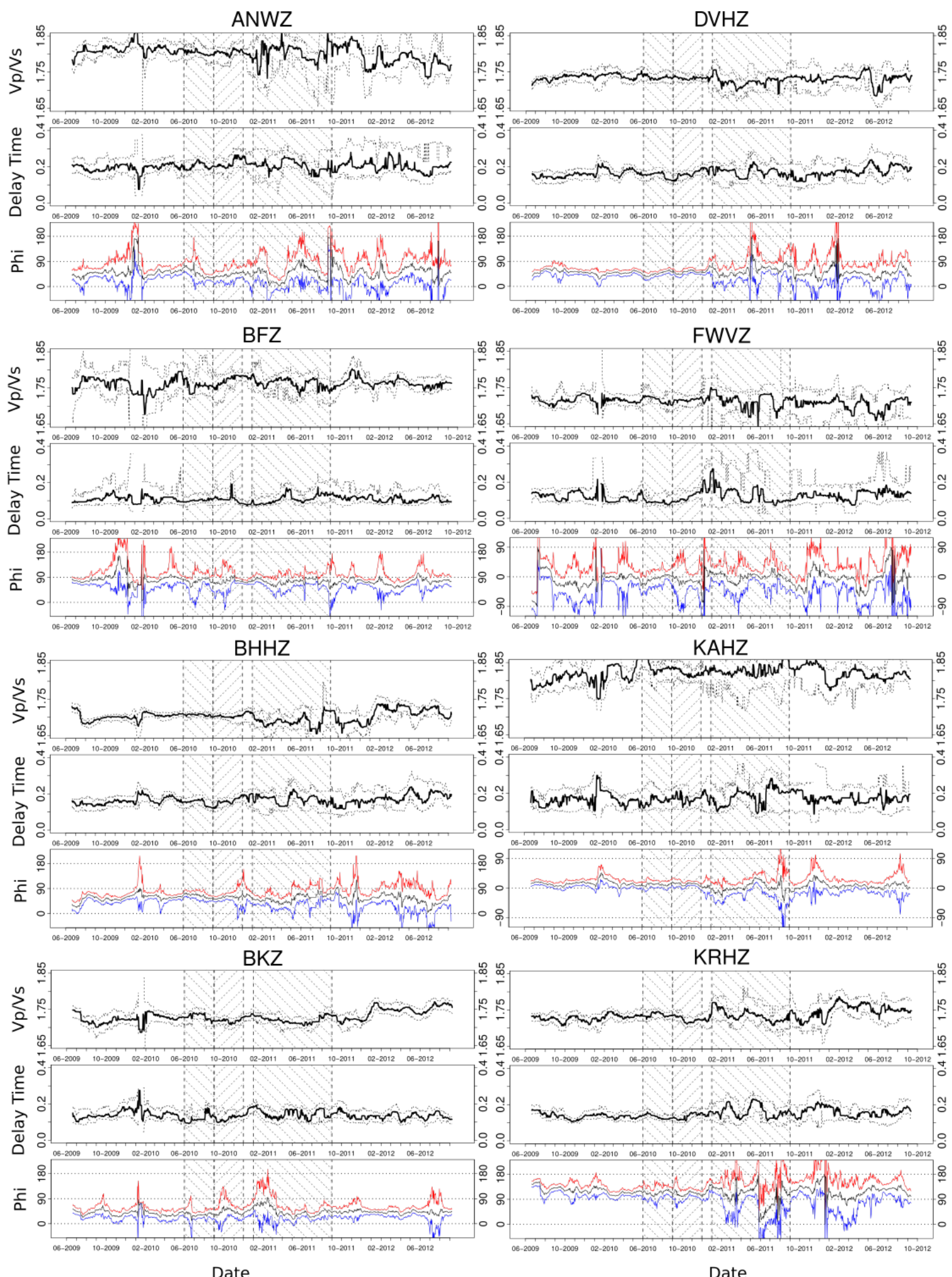

Figure C.1: 3 part figure. Shear wave splitting and $\mathrm{Vp} / \mathrm{Vs}$ results versus time for each station used in this study. Vp/Vs and Delay time: Black line is a median of 40 day moving windows with $95 \%$ confidence intervals. The 3 stages of the 2010 Manawatu SSE are shaded with diagonal dotted lines. Phi: Fast polarization directions. Black line is a circular mean of 40 day moving windows with $95 \%$ confidence intervals. 

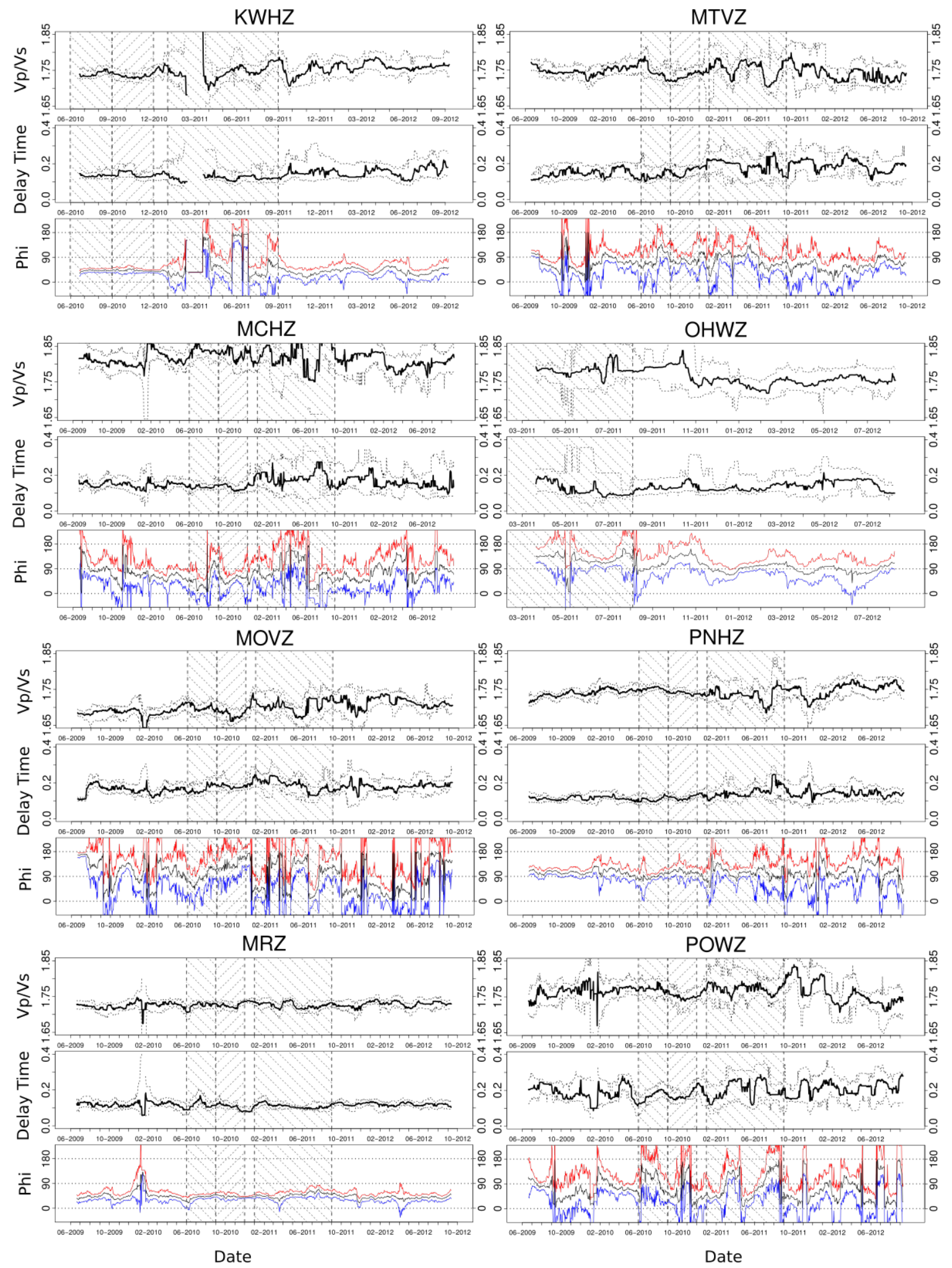

Figure C.1: (B) continued. 


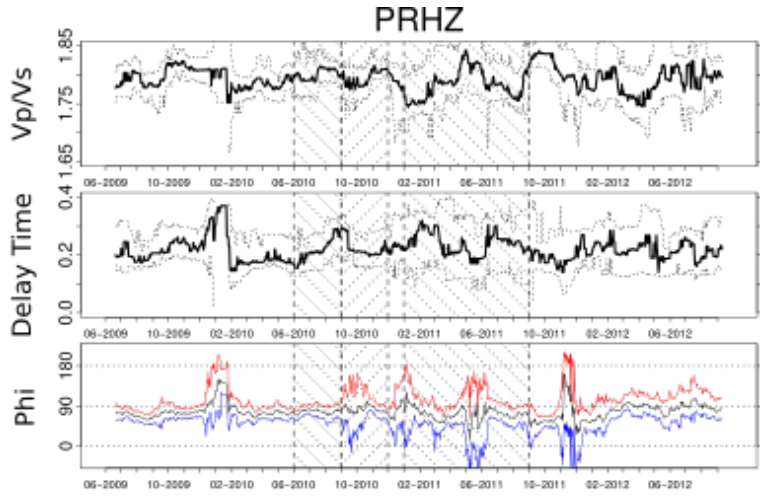

PRWZ

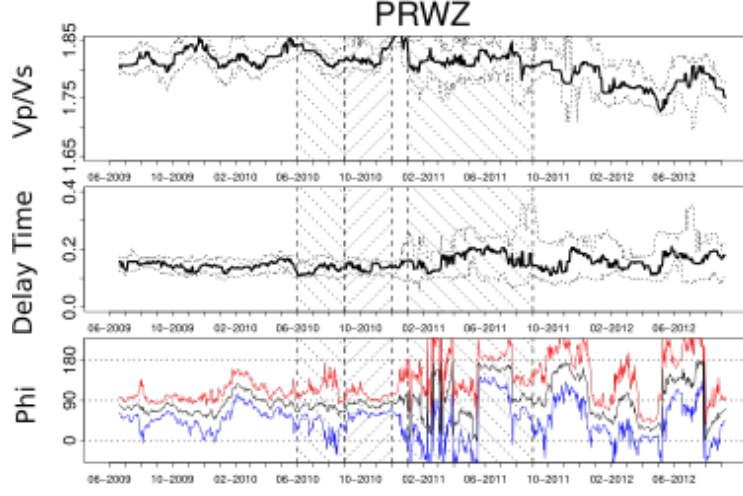

PXZ

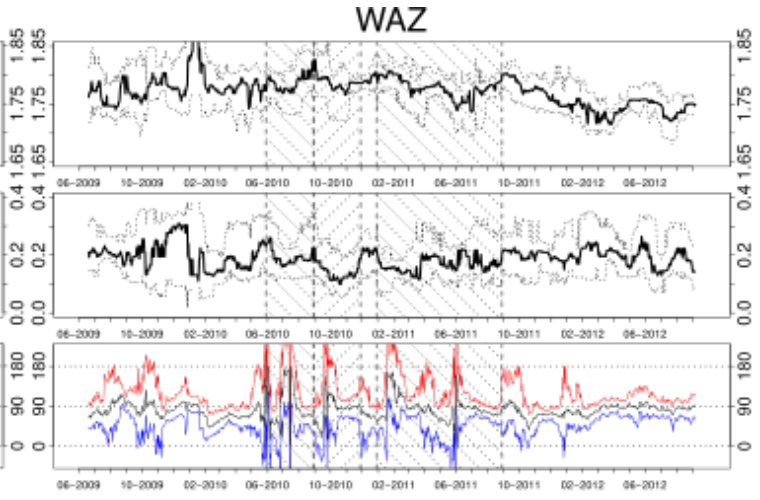

WHVZ

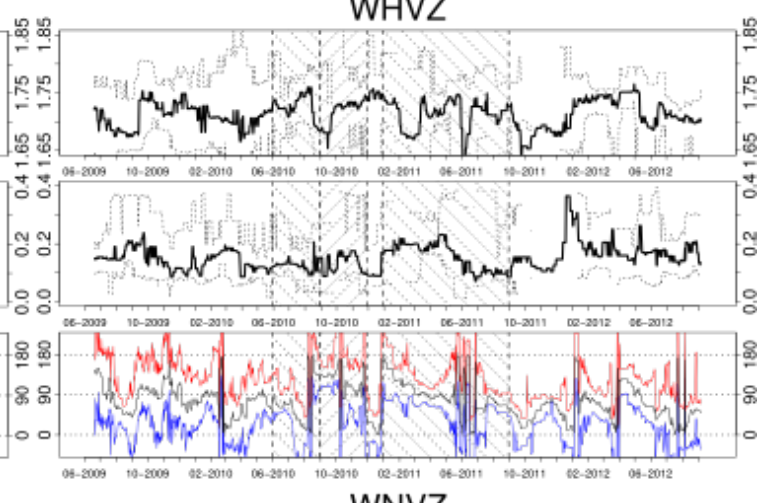

WNVZ
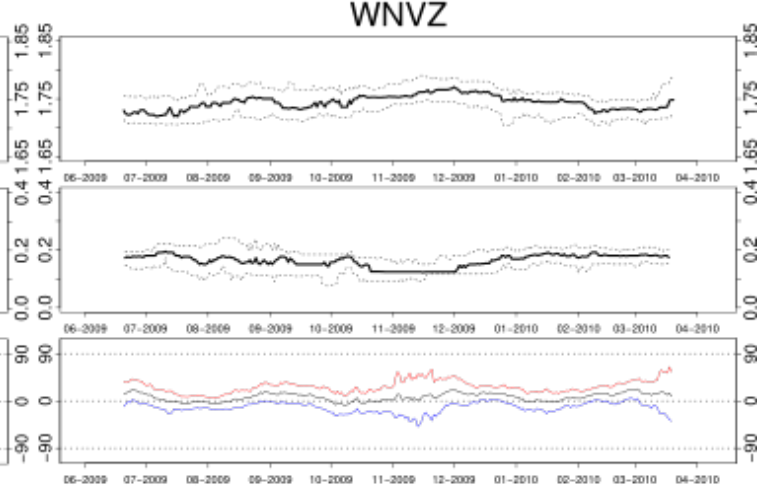

TSZ

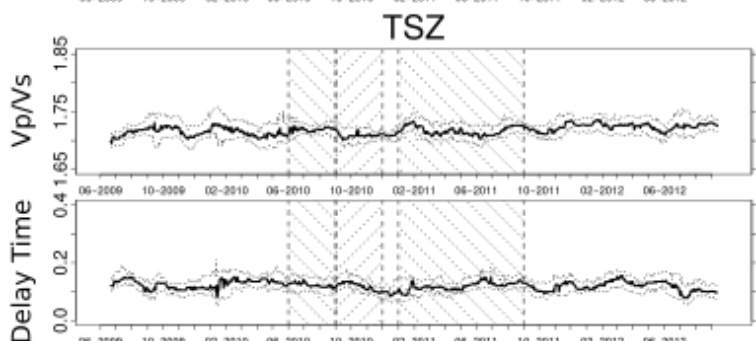

工立。

Date

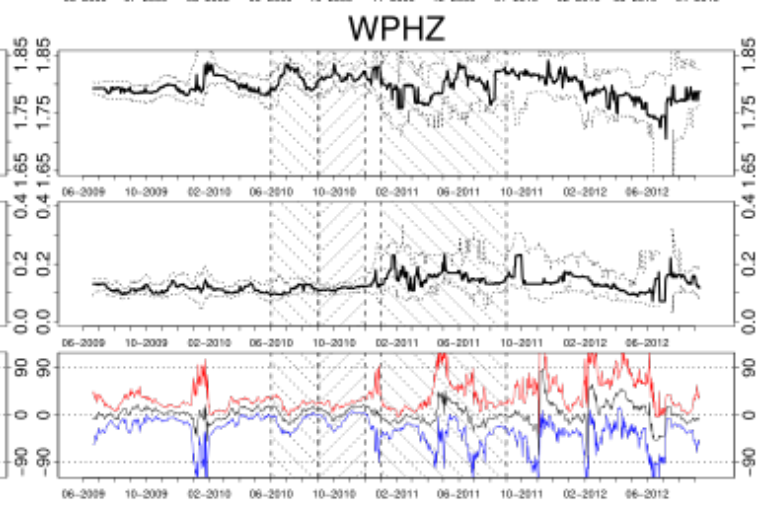

Date

Figure C.1: (C) continued. 

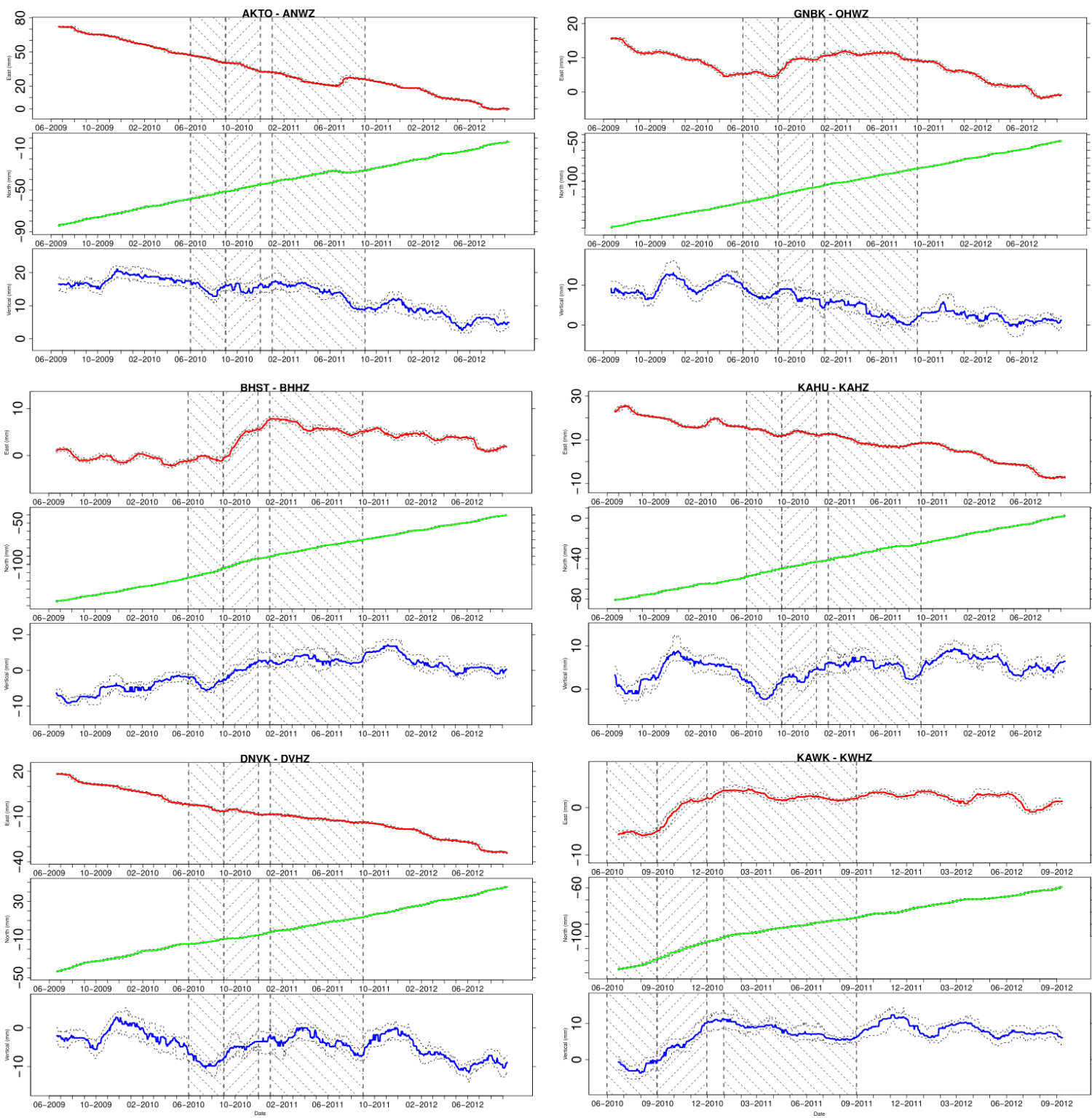

Figure C.2: 3 part figure. cGPS time series for GeoNet GPS sites corresponding to the GeoNet seismic stations used in this study. The title of each plot lists the cGPS station name first, followed by the name of the corresponding seismic station shown in Figure C.1. Displacements are shown on the east (red), north (green) and vertical (blue) components. East line is a median of 40 day moving windows with 95\% confidence intervals. The 3 stages of the 2010 Manawatu SSE are shaded with diagonal dotted lines. 

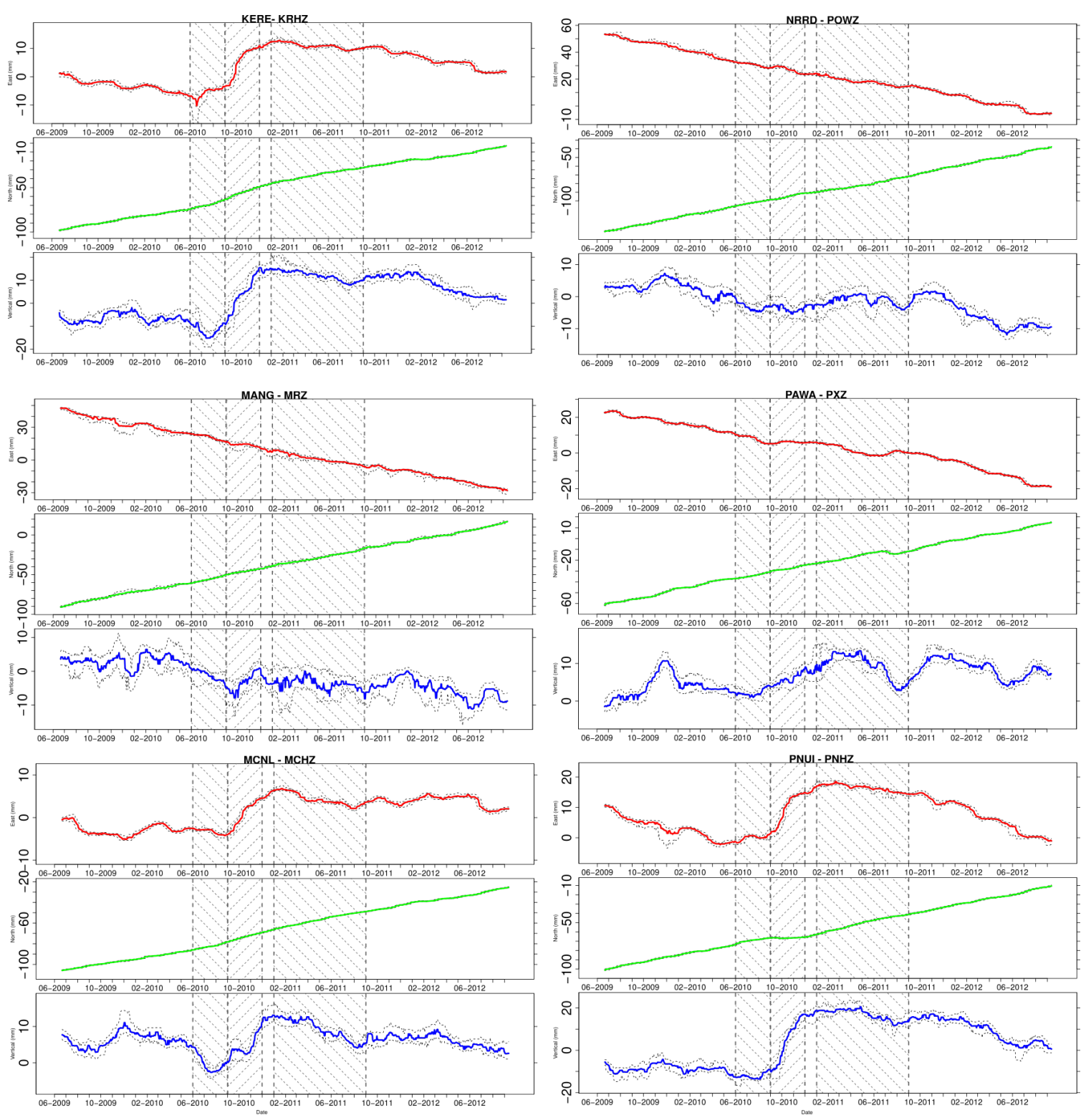

Figure C.2: (B) continued. 

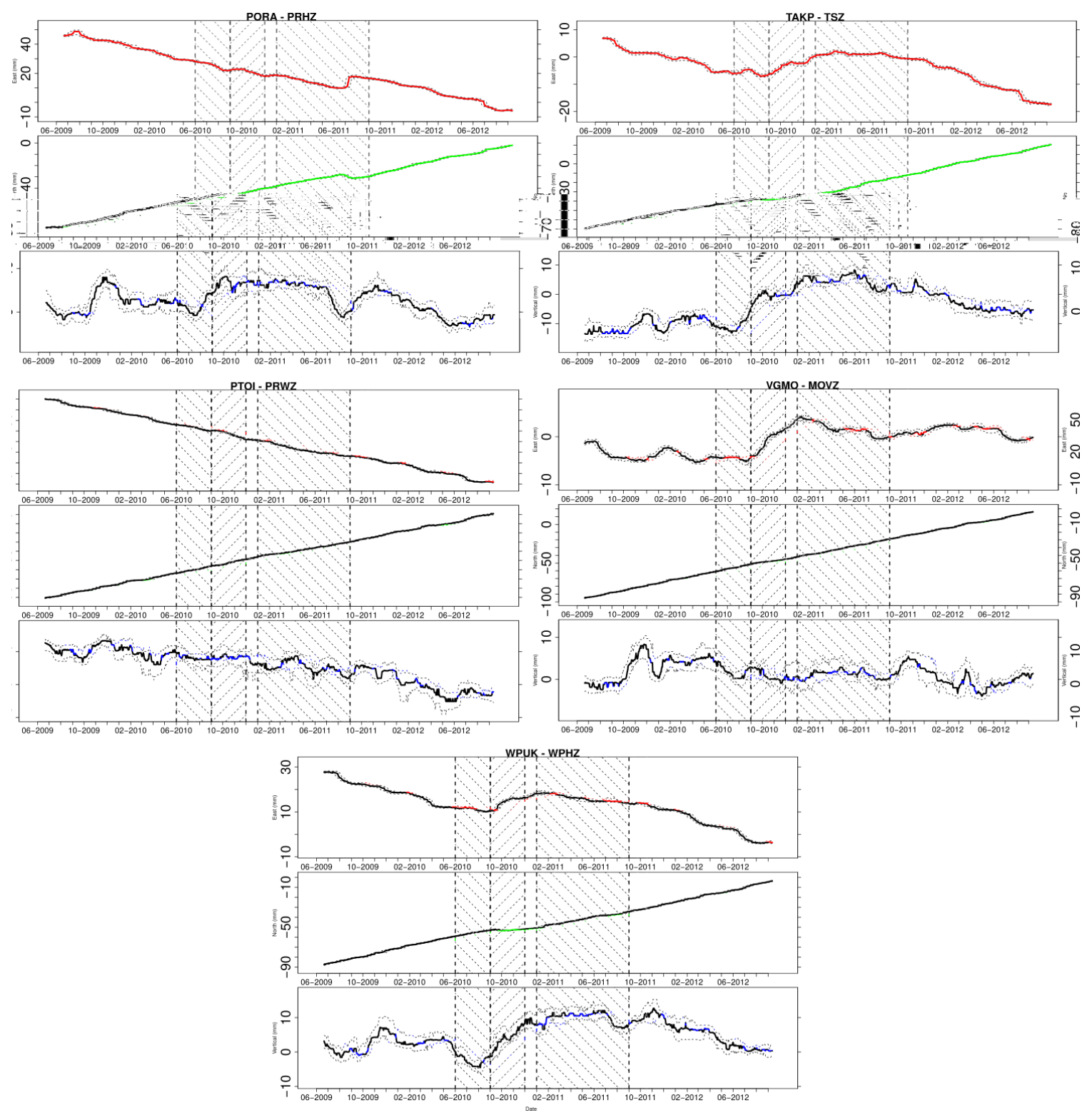

Figure C.2: (C) continued. 

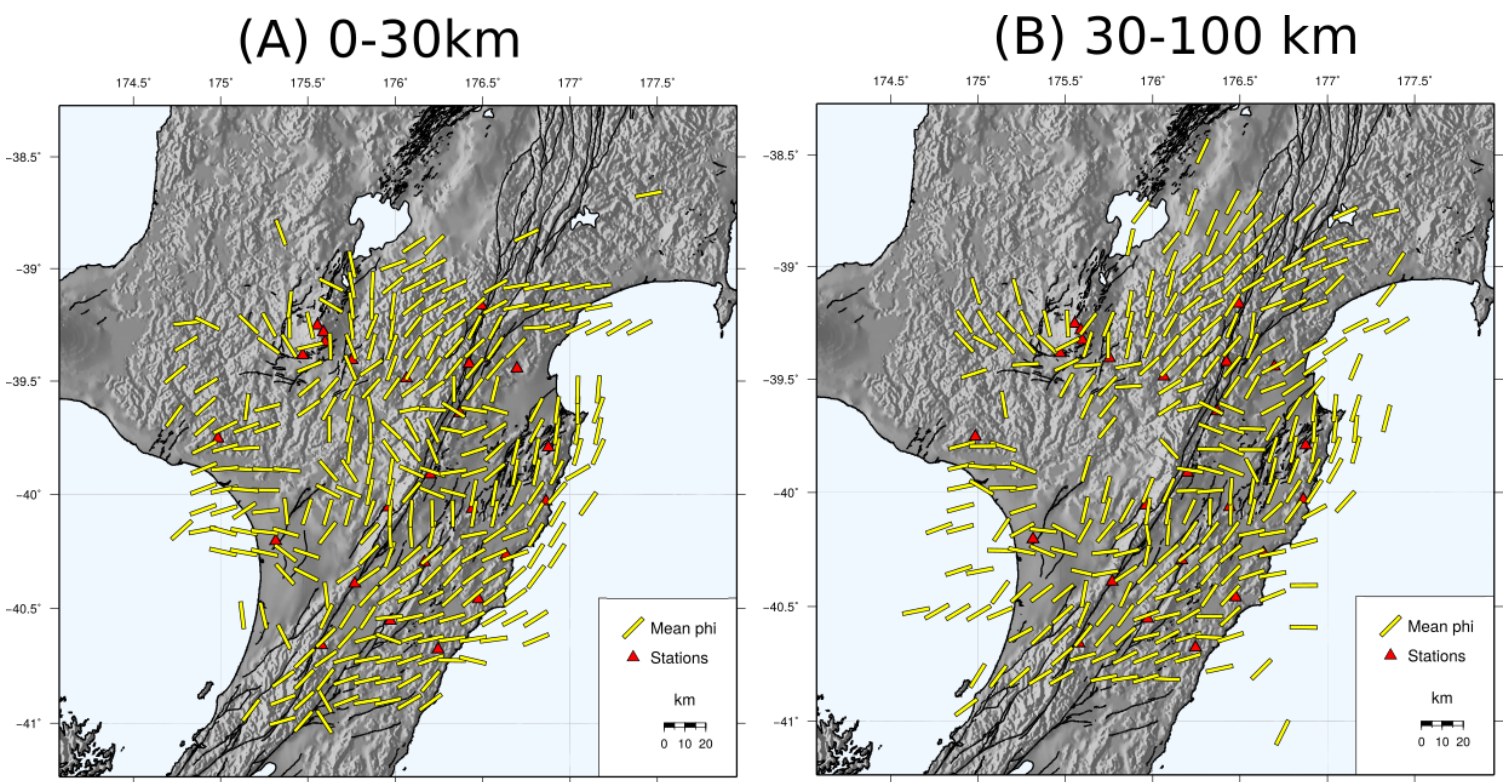

Figure C.3: Spatial averages of SWS fast polarization azimuths from TESSA Johnson et al. (2011) comparing earthquakes between (A) 0-30 km depth and (B) 30-100 km depth. Yellow bars show average fast polarization azimuths for each block plotted in the center of the grid block. Fast polarization azimuths are not plotted for blocks with average values that have a standard deviation greater than $30^{\circ}$ and standard error larger than $10^{\circ}$. Red triangles mark locations of seismic stations used in this study. 

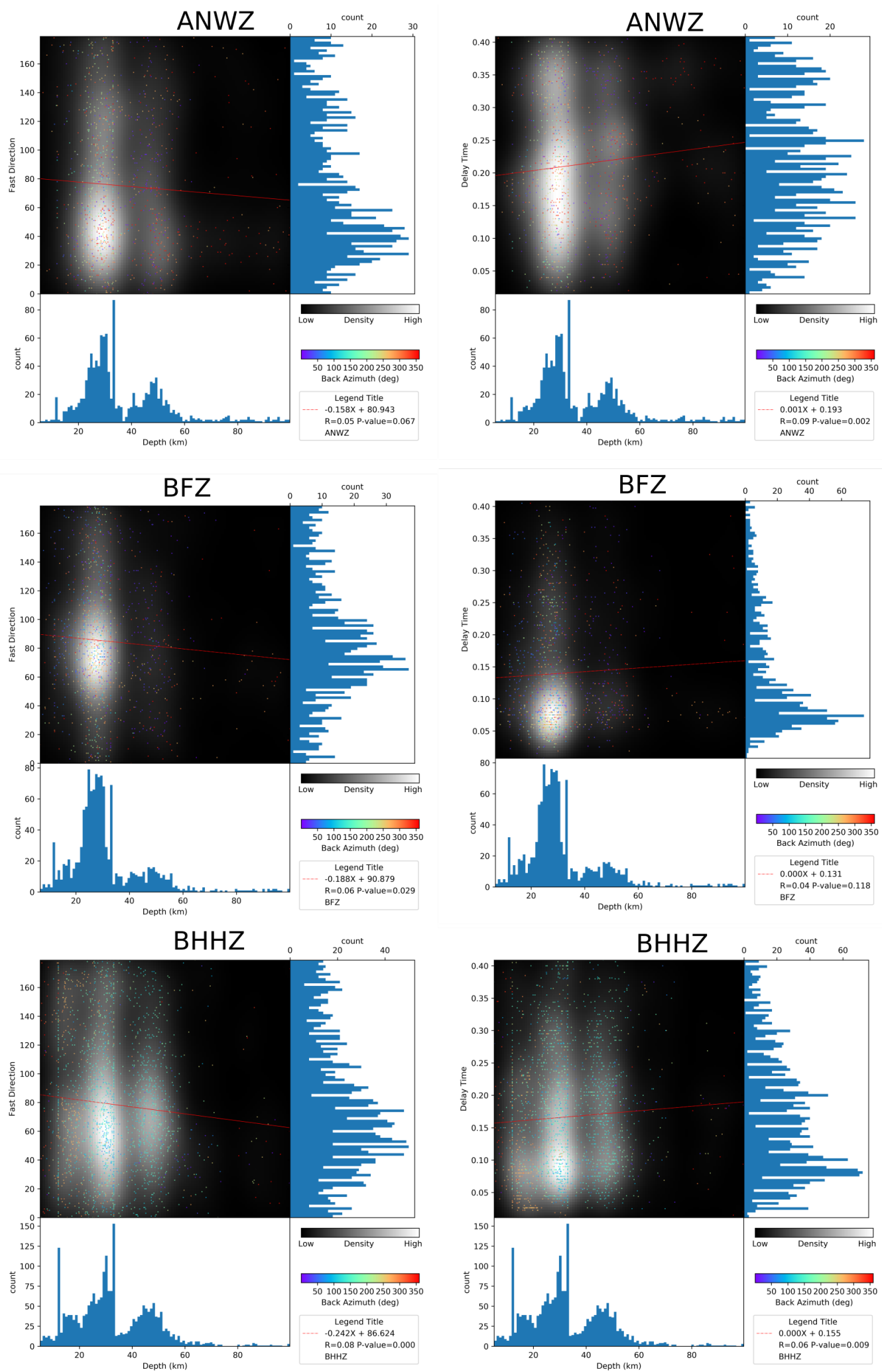

Figure C.4: 8 part figure for the 24 stations used in this study. Left column: Fast polarization directions versus depth. Individual measurements are coloured by their event-station back-azimuth. $X$ and $Y$ axes show histograms of measurement count at varying fast direction and depth. Central plot is coloured by the density of measurements. A linear regression line is fitted to highlight variations with depth. Right column: Delay time versus depth. 

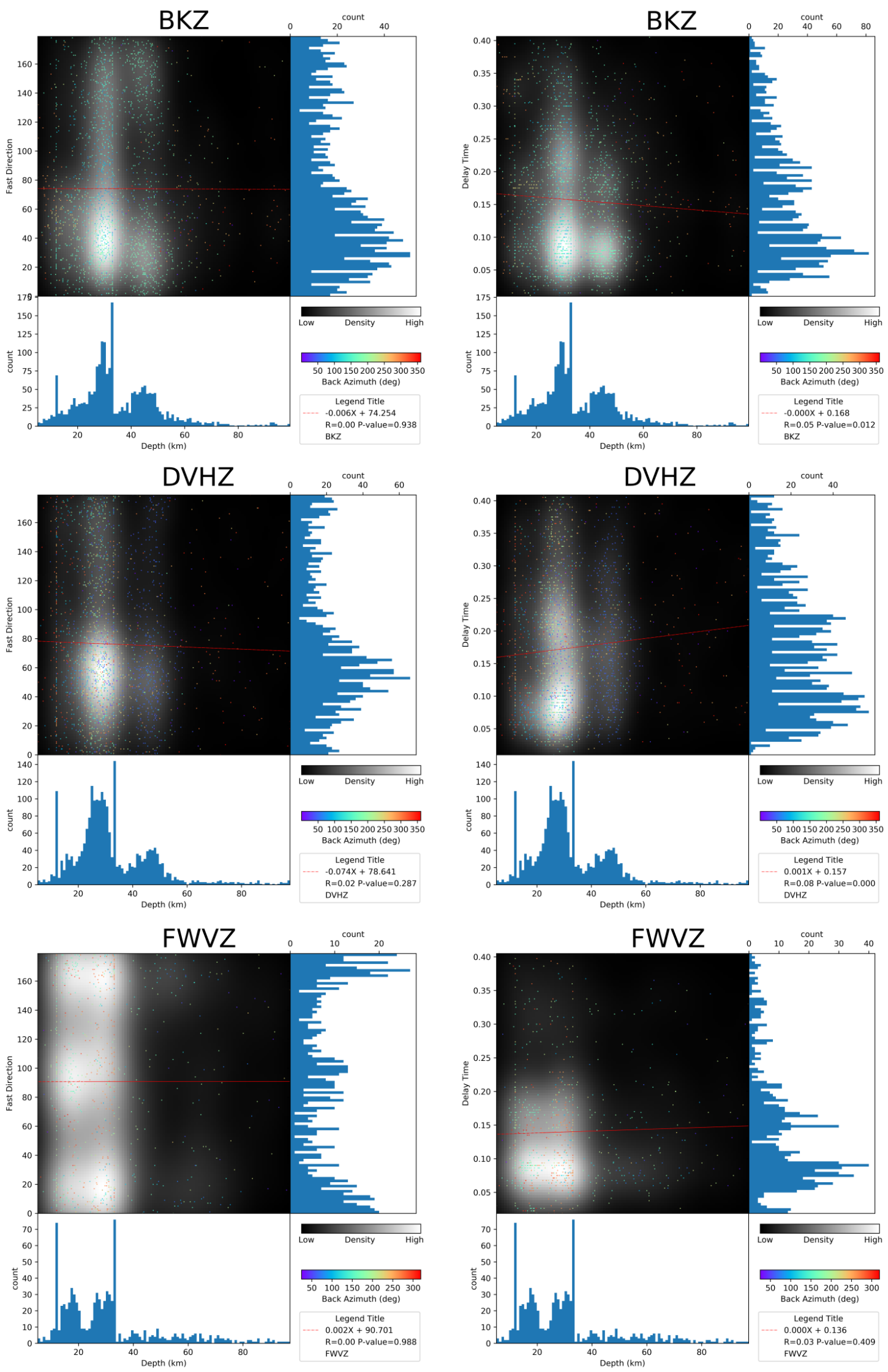

Figure C.4: (B) continued. 

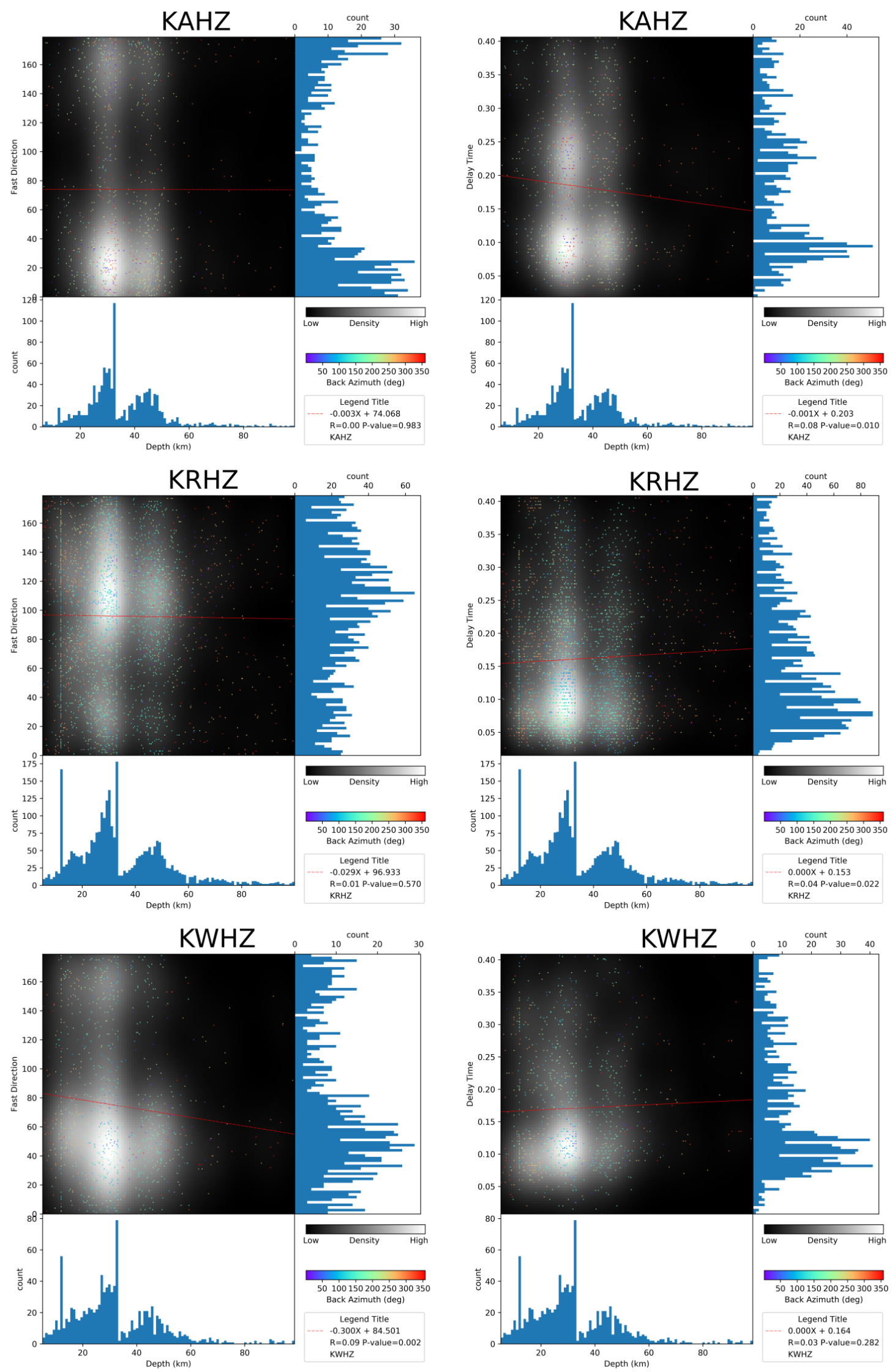

Figure C.4: (C) continued. 

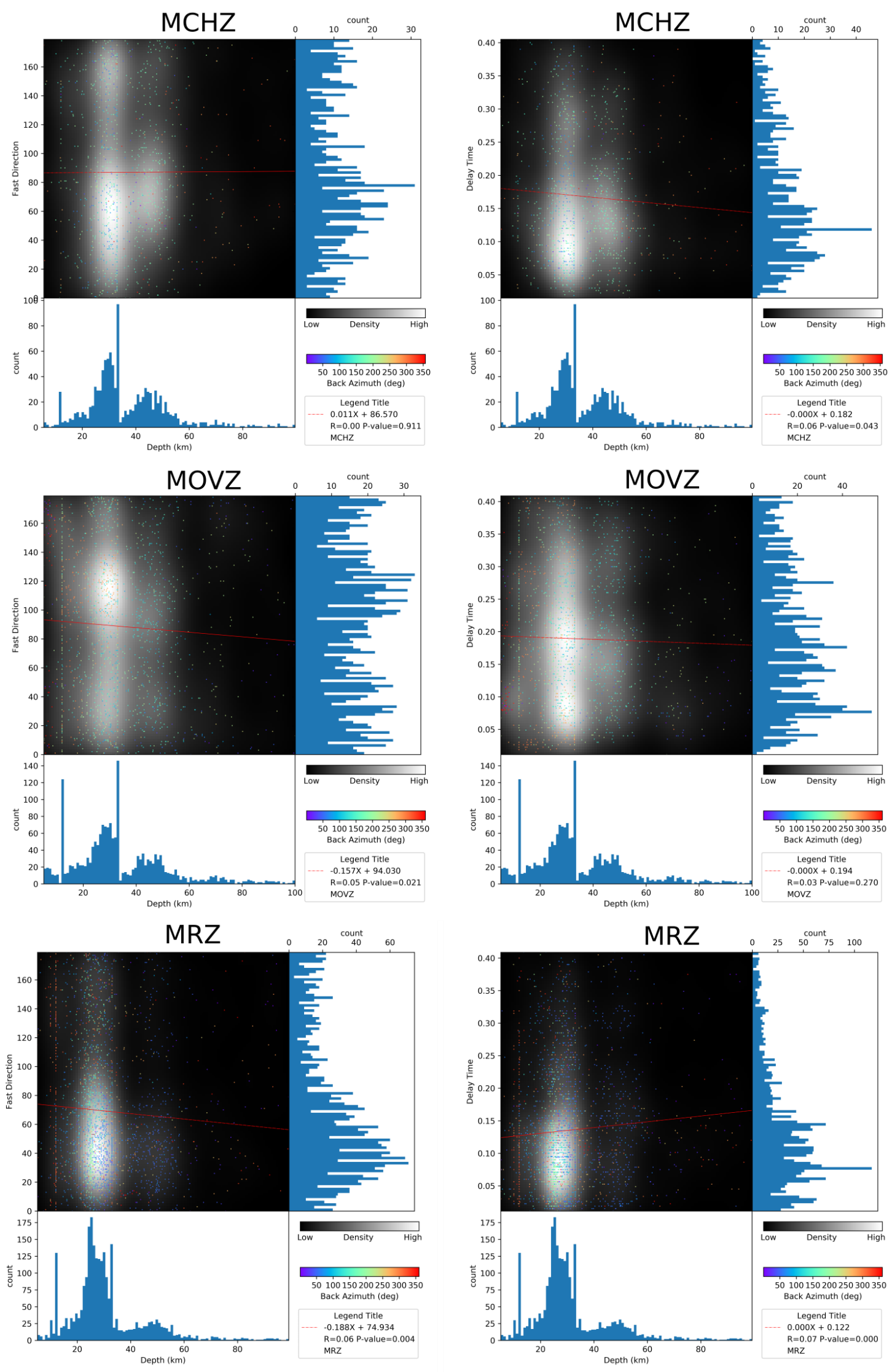

Figure C.4: (D) continued. 

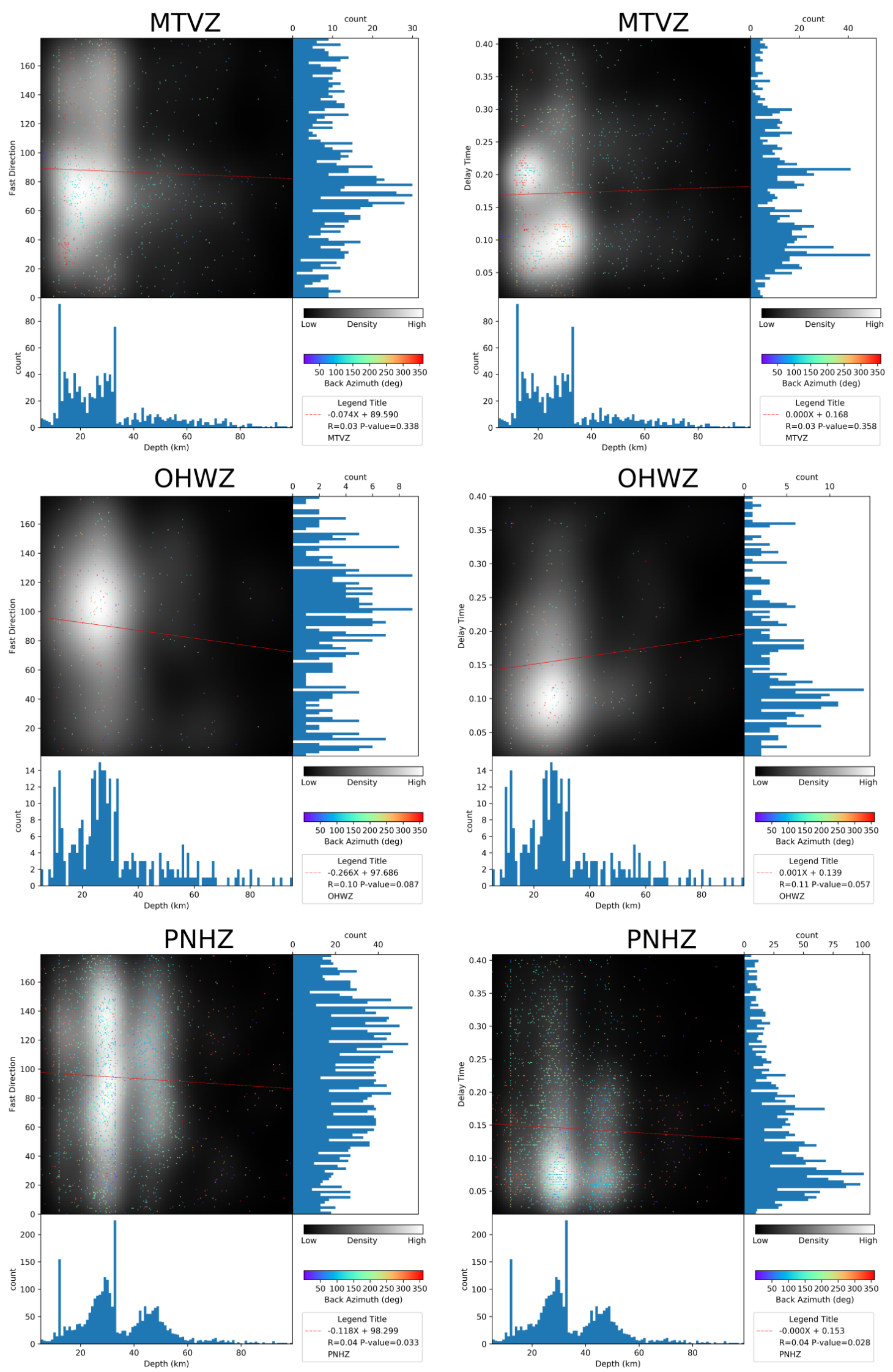

Figure C.4: (E) continued. 

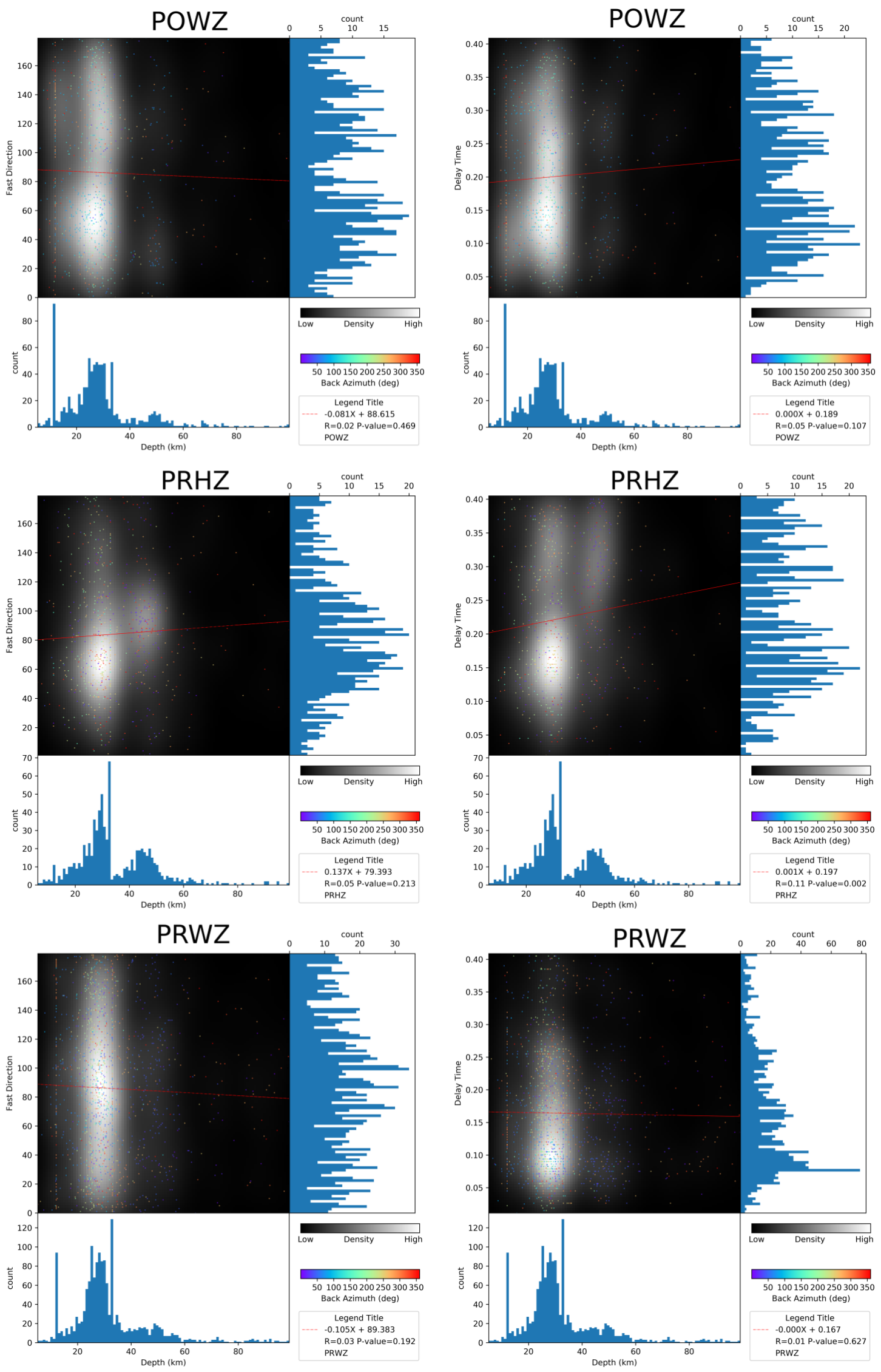

Figure C.4: (F) continued. 

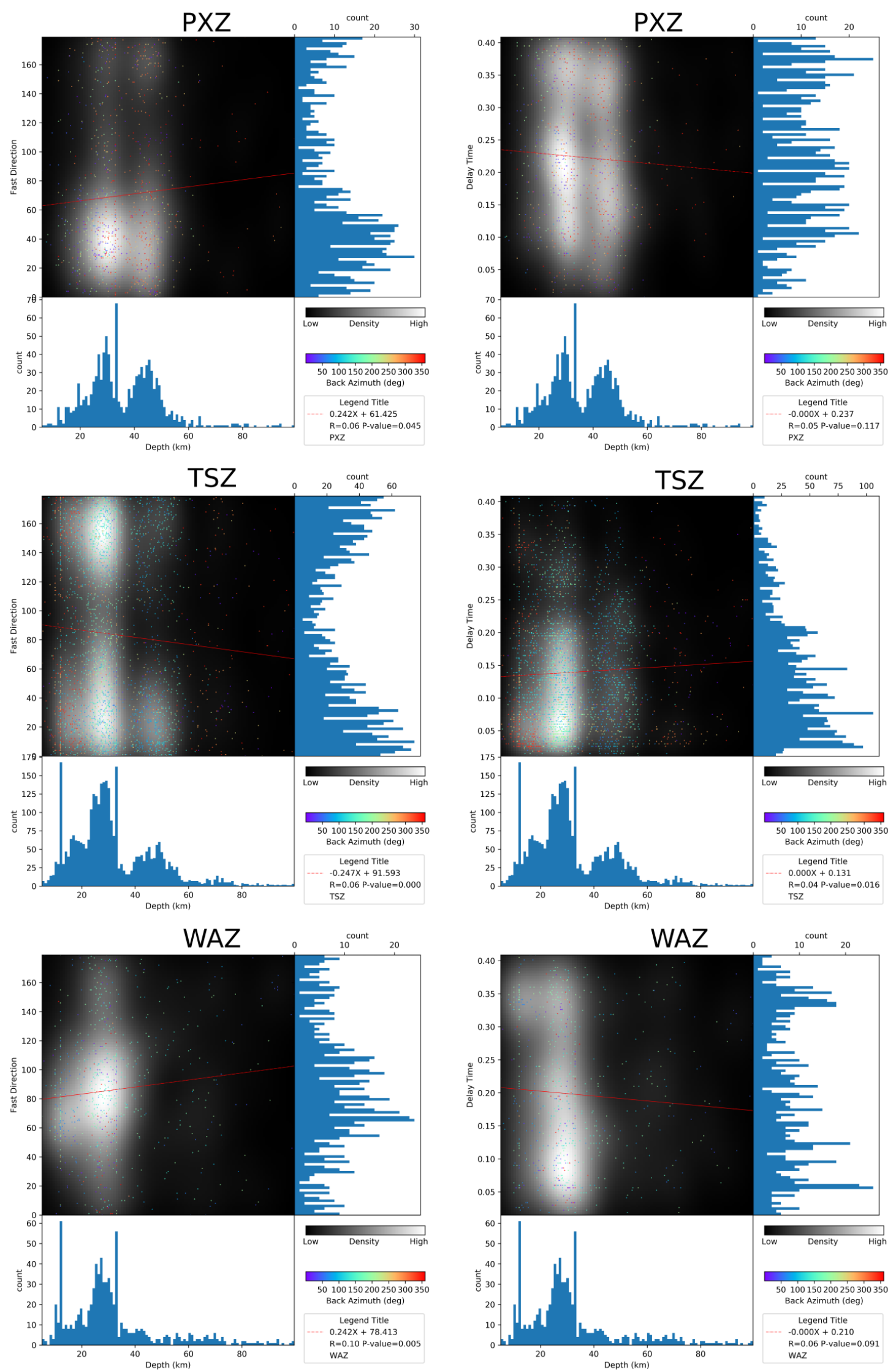

Figure C.4: (G) continued. 

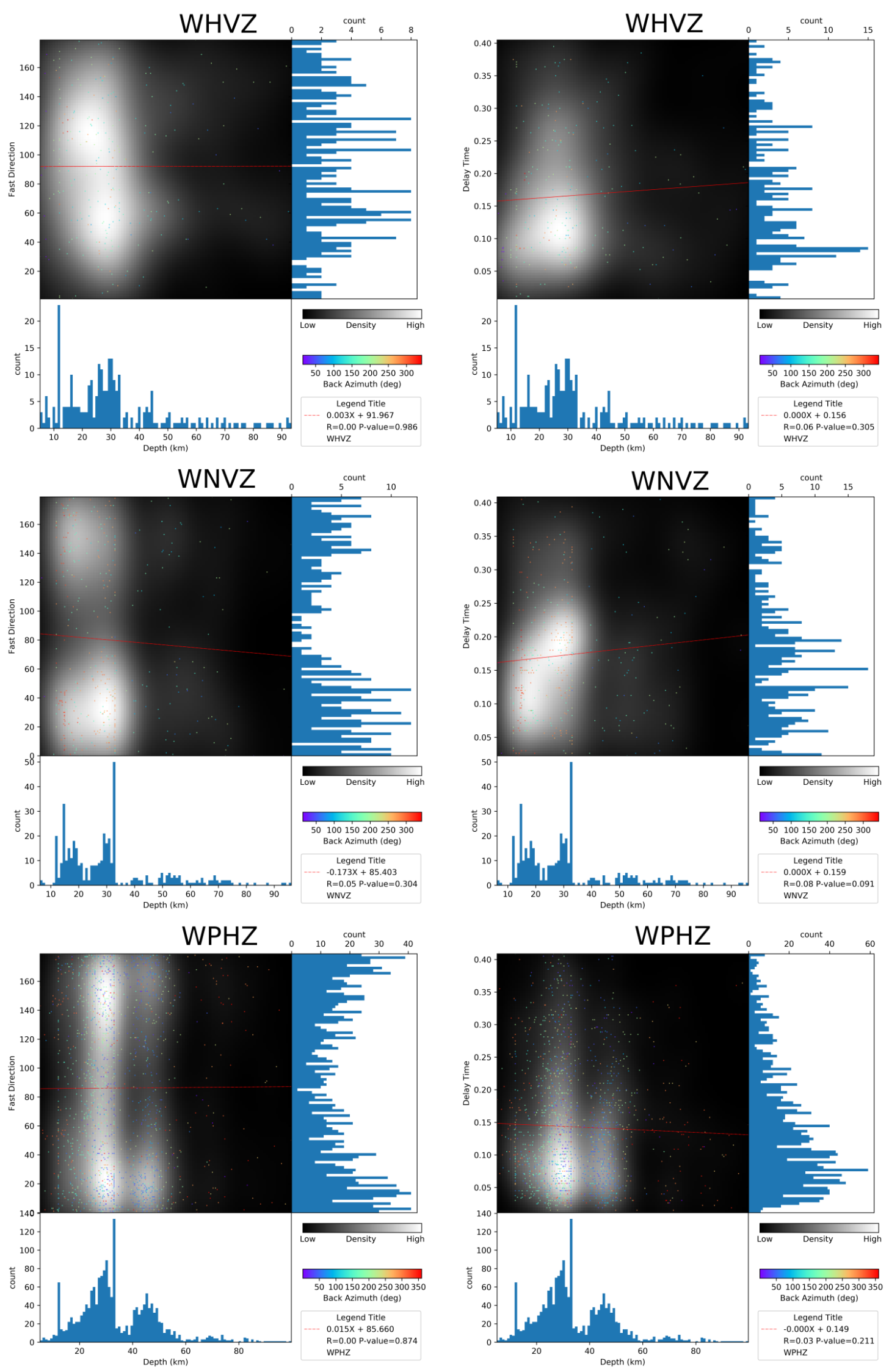

Figure C.4: (H) continued. 


\section{Appendix D}

\section{Appendix D: Chapter 5}

This chapter provides appendix material for Chapter 5: Upper mantle anisotropy across the Taranaki-Ruapehu Line in North Island, New Zealand. 
Table D.1: Individual SWS results on RATTIL stations.

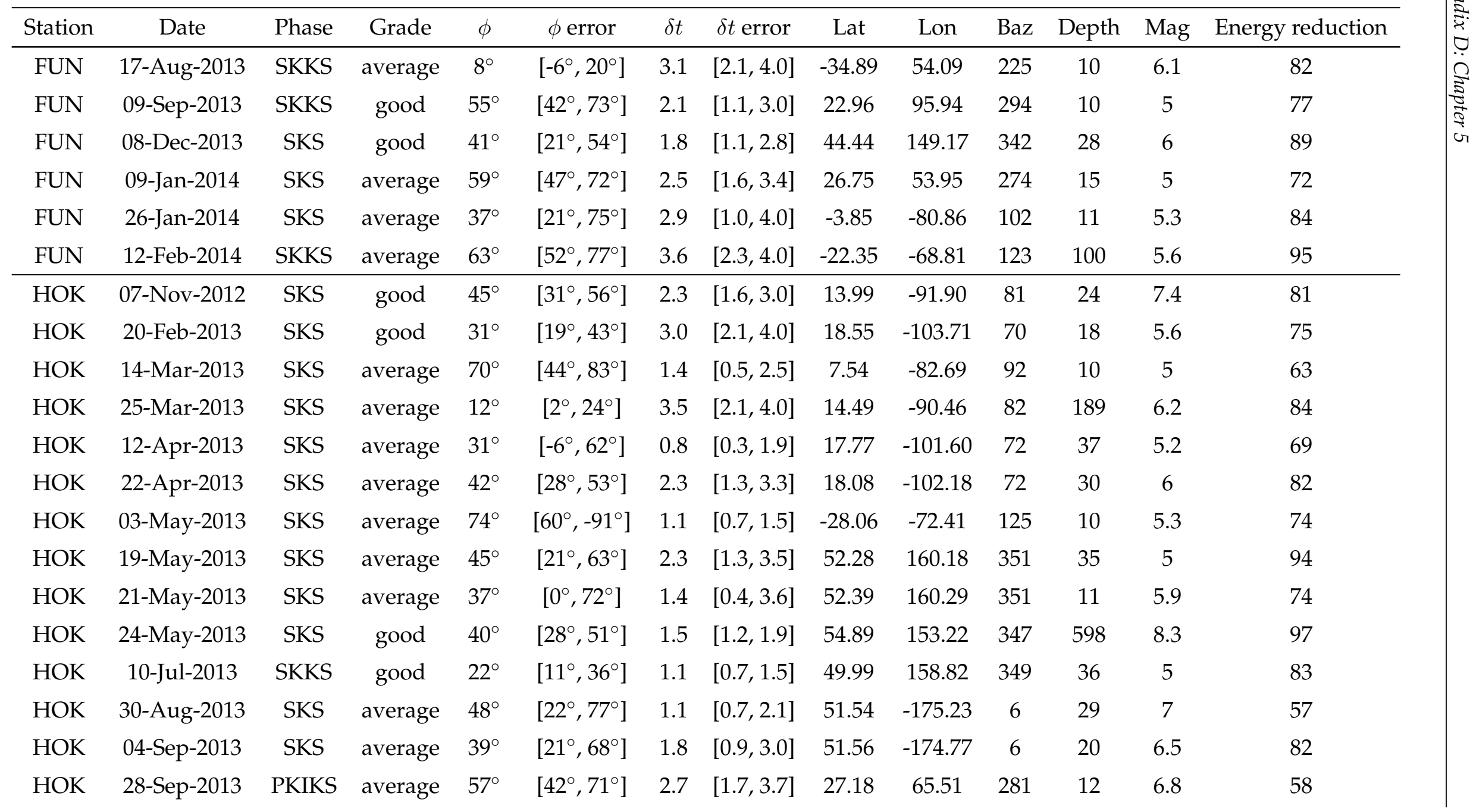


Table D.1 continued from previous page

\begin{tabular}{|c|c|c|c|c|c|c|c|c|c|c|c|c|c|}
\hline Station & Date & Phase & Grade & $\phi$ & $\phi$ error & $\delta t$ & $\delta t$ error & Lat & Lon & Baz & Depth & Mag & Energy reduction \\
\hline HOK & 01-Oct-2013 & SKS & average & $62^{\circ}$ & {$\left[45^{\circ}, 69^{\circ}\right]$} & 1.9 & {$[0.8,3.3]$} & 53.20 & 152.79 & 347 & 573 & 6.7 & 83 \\
\hline $\mathrm{HOK}$ & 28-Oct-2013 & SKKS & average & $-73^{\circ}$ & {$\left[-87^{\circ},-44^{\circ}\right]$} & 1.1 & {$[0.4,2.2]$} & 76.28 & 7.12 & 355 & 10 & 5.3 & 63 \\
\hline HOK & 25-Nov-2013 & SKS & average & $63^{\circ}$ & {$\left[43^{\circ}, 81^{\circ}\right]$} & 1.4 & {$[0.7,2.3]$} & -3.17 & -79.89 & 102 & 85 & 5 & 76 \\
\hline HOK & 13-Jan-2014 & SKKS & average & $68^{\circ}$ & {$\left[50^{\circ}, 81^{\circ}\right]$} & 2.4 & {$[1.2,3.8]$} & 19.04 & -66.81 & 92 & 20 & 6.4 & 74 \\
\hline $\mathrm{HOK}$ & 23-Feb-2014 & SKS & average & $21^{\circ}$ & {$\left[1^{\circ}, 42^{\circ}\right]$} & 1.5 & {$[0.7,2.4]$} & 43.51 & 147.77 & 340 & 20 & 5.3 & 67 \\
\hline HOK & 24-Feb-2014 & SKKS & average & $14^{\circ}$ & {$\left[7^{\circ}, 22^{\circ}\right]$} & 3.4 & {$[2.7,4.0]$} & 4.13 & 62.63 & 258 & 10 & 5.6 & 94 \\
\hline KUR & 07-Nov-2012 & SKS & good & $14^{\circ}$ & {$\left[5^{\circ}, 30^{\circ}\right]$} & 2.7 & {$[1.6,3.9]$} & 13.99 & -91.90 & 81 & 24 & 7.4 & 96 \\
\hline KUR & 09-Feb-2013 & SKS & average & $54^{\circ}$ & {$\left[21^{\circ}, 89^{\circ}\right]$} & 0.7 & {$[0.4,2.1]$} & 1.14 & -77.39 & 100 & 145 & 6.9 & 86 \\
\hline KUR & 09-Feb-2013 & SKIKS & average & $65^{\circ}$ & {$\left[33^{\circ}, 87^{\circ}\right]$} & 0.8 & {$[0.5,1.7]$} & 1.14 & -77.39 & 100 & 145 & 6.9 & 92 \\
\hline KUR & 28-Feb-2013 & SKS & average & $52^{\circ}$ & {$\left[27^{\circ}, 66^{\circ}\right]$} & 2.1 & {$[1.1,3.5]$} & 50.95 & 157.28 & 349 & 41 & 6.9 & 90 \\
\hline KUR & 01-Mar-2013 & SKS & average & $21^{\circ}$ & {$\left[5^{\circ}, 46^{\circ}\right]$} & 1.8 & {$[1.0,2.6]$} & 50.90 & 157.45 & 349 & 33 & 6.4 & 72 \\
\hline KUR & 06-Apr-2013 & SKIKS & average & $38^{\circ}$ & {$\left[29^{\circ}, 47^{\circ}\right]$} & 2.7 & {$[2.1,3.4]$} & 34.80 & 24.09 & 268 & 34 & 5.3 & 82 \\
\hline KUR & 19-Apr-2013 & SKS & average & $57^{\circ}$ & {$\left[51^{\circ}, 62^{\circ}\right]$} & 3.8 & {$[2.7,4.0]$} & 46.22 & 150.79 & 343 & 110 & 7.2 & 90 \\
\hline MOT & 07-Nov-2012 & SKS & good & $46^{\circ}$ & {$\left[36^{\circ}, 56^{\circ}\right]$} & 3.4 & {$[2.6,4.0]$} & 13.99 & -91.90 & 81 & 24 & 7.4 & 96 \\
\hline MOT & 27-Dec-2012 & SKIKS & average & $26^{\circ}$ & {$\left[20^{\circ}, 40^{\circ}\right]$} & 1.0 & {$[0.4,1.6]$} & 13.01 & -58.00 & 104 & 10 & 5.3 & 50 \\
\hline MOT & 09-Feb-2013 & SKS & average & $30^{\circ}$ & {$\left[19^{\circ}, 62^{\circ}\right]$} & 2.1 & {$[0.7,3.8]$} & 1.14 & -77.39 & 100 & 145 & 6.9 & 79 \\
\hline MOT & 19-Apr-2013 & SKS & average & $57^{\circ}$ & {$\left[22^{\circ}, 66^{\circ}\right]$} & 1.1 & {$[0.4,2.2]$} & 46.22 & 150.79 & 343 & 110 & 7.2 & 93 \\
\hline MOT & 29-Dec-2013 & SKKS & average & $36^{\circ}$ & {$\left[26^{\circ}, 52^{\circ}\right]$} & 1.9 & {$[1.1,3.0]$} & 41.37 & 14.45 & 284 & 11 & 5.1 & 73 \\
\hline $\mathrm{OIO}$ & 06-Mar-2013 & SKKS & good & $79^{\circ}$ & {$\left[57^{\circ},-83^{\circ}\right]$} & 1.3 & {$[0.7,1.9]$} & -43.47 & 39.99 & 211 & 10 & 5.2 & 73 \\
\hline $\mathrm{OIO}$ & 25-Mar-2013 & SKS & good & $42^{\circ}$ & {$\left[23^{\circ}, 59^{\circ}\right]$} & 2.3 & {$[1.4,3.2]$} & 14.49 & -90.46 & 82 & 189 & 6.2 & 88 \\
\hline $\mathrm{OIO}$ & 02-Jun-2013 & SKS & average & $-61^{\circ}$ & {$\left[-81^{\circ},-33^{\circ}\right]$} & 1.1 & {$[0.5,1.8]$} & 12.32 & -88.23 & 85 & 35 & 5 & 62 \\
\hline
\end{tabular}


Table D.1 continued from previous page

\begin{tabular}{|c|c|c|c|c|c|c|c|c|c|c|c|c|c|}
\hline Station & Date & Phase & Grade & $\phi$ & $\phi$ error & $\delta t$ & $\delta t$ error & Lat & Lon & Baz & Depth & Mag & Energy reduction \\
\hline $\mathrm{OIO}$ & 08-Jun-2013 & SKS & average & $13^{\circ}$ & {$\left[-39^{\circ}, 30^{\circ}\right]$} & 0.7 & {$[0.1,1.7]$} & -22.59 & -66.69 & 125 & 215 & 5.6 & 52 \\
\hline $\mathrm{OIO}$ & 01-Oct-2013 & SKS & average & $64^{\circ}$ & {$\left[48^{\circ}, 71^{\circ}\right]$} & 2.1 & {$[0.8,3.6]$} & 53.20 & 152.79 & 347 & 573 & 6.7 & 79 \\
\hline PAR & 07-Nov-2012 & SKS & good & $28^{\circ}$ & {$\left[17^{\circ}, 41^{\circ}\right]$} & 1.7 & {$[1.3,2.3]$} & 13.99 & -91.90 & 81 & 24 & 7.4 & 91 \\
\hline PAR & 16-Nov-2012 & SKS & average & $46^{\circ}$ & {$\left[31^{\circ}, 58^{\circ}\right]$} & 2.5 & {$[1.6,3.5]$} & 49.28 & 155.43 & 347 & 29 & 6.5 & 95 \\
\hline PAR & 02-Feb-2013 & SKS & good & $36^{\circ}$ & {$\left[10^{\circ}, 51^{\circ}\right]$} & 1.4 & {$[0.9,2.4]$} & 42.77 & 143.09 & 337 & 107 & 6.9 & 93 \\
\hline PAR & 09-Feb-2013 & SKS & good & $39^{\circ}$ & {$\left[24^{\circ}, 68^{\circ}\right]$} & 1.5 & {$[0.8,2.7]$} & 1.14 & -77.39 & 101 & 145 & 6.9 & 80 \\
\hline PAR & 05-Mar-2013 & SKIKS & average & $-53^{\circ}$ & {$\left[-76^{\circ},-34^{\circ}\right]$} & 1.7 & {$[0.9,2.7]$} & 67.64 & 142.42 & 347 & 10 & 5.2 & 76 \\
\hline PAR & 25-Mar-2013 & SKS & average & $29^{\circ}$ & {$\left[7^{\circ}, 58^{\circ}\right]$} & 1.5 & {$[0.8,3.0]$} & 14.49 & -90.46 & 82 & 189 & 6.2 & 71 \\
\hline PAR & 19-Apr-2013 & SKS & good & $47^{\circ}$ & {$\left[31^{\circ}, 58^{\circ}\right]$} & 2.1 & {$[1.2,3.0]$} & 46.22 & 150.79 & 343 & 110 & 7.2 & 94 \\
\hline PAR & 19-Мay-2013 & SKKS & average & $-51^{\circ}$ & {$\left[-70^{\circ},-35^{\circ}\right]$} & 0.7 & {$[0.5,1.0]$} & 56.17 & 164.41 & 354 & 21 & 5 & 80 \\
\hline PAR & 24-May-2013 & SKS & average & $35^{\circ}$ & {$\left[10^{\circ}, 56^{\circ}\right]$} & 1.5 & {$[0.9,2.5]$} & 52.24 & 151.44 & 346 & 624 & 6.7 & 84 \\
\hline PAR & 24-Мау-2013 & SKS & average & $54^{\circ}$ & {$\left[25^{\circ}, 67^{\circ}\right]$} & 1.7 & {$[0.8,3.3]$} & 54.89 & 153.22 & 347 & 598 & 8.3 & 86 \\
\hline PAR & 28-May-2013 & SKKS & average & $68^{\circ}$ & {$\left[54^{\circ}, 82^{\circ}\right]$} & 1.3 & {$[0.9,1.7]$} & 43.25 & 41.66 & 291 & 5 & 5.1 & 86 \\
\hline PAR & 13-Aug-2013 & SKS & average & $-69^{\circ}$ & {$\left[81^{\circ},-37^{\circ}\right]$} & 0.8 & {$[0.3,1.4]$} & 43.72 & 143.47 & 338 & 178 & 5.1 & 71 \\
\hline PAR & 28-Sep-2013 & SKKS & average & $-41^{\circ}$ & {$\left[-65^{\circ},-14^{\circ}\right]$} & 1.5 & {$[0.6,2.5]$} & 27.18 & 65.51 & 281 & 12 & 6.8 & 67 \\
\hline PAR & 01-Oct-2013 & SKS & good & $44^{\circ}$ & {$\left[21^{\circ}, 59^{\circ}\right]$} & 1.8 & {$[1.1,2.9]$} & 53.20 & 152.79 & 347 & 573 & 6.7 & 92 \\
\hline PAR & 02-Oct-2013 & SKS & average & $-37^{\circ}$ & {$\left[-54^{\circ},-24^{\circ}\right]$} & 2.1 & {$[1.2,2.8]$} & 0.47 & -89.54 & 93 & 10 & 5.3 & 93 \\
\hline PAR & 21-Oct-2013 & SKS & average & $-48^{\circ}$ & {$\left[-62^{\circ},-34^{\circ}\right]$} & 2.4 & {$[1.5,3.2]$} & 51.70 & -175.31 & 6 & 13 & 5 & 87 \\
\hline PAR & 08-Dec-2013 & SKS & average & $23^{\circ}$ & {$\left[-2^{\circ}, 51^{\circ}\right]$} & 1.3 & {$[0.6,2.3]$} & 44.44 & 149.17 & 342 & 28 & 6 & 69 \\
\hline PEP & 07-Nov-2012 & SKS & good & $28^{\circ}$ & {$\left[12^{\circ}, 49^{\circ}\right]$} & 2.2 & {$[1.4,3.1]$} & 13.99 & -91.90 & 81 & 24 & 7.4 & 81 \\
\hline PEP & 11-Nov-2012 & SKKS & average & $56^{\circ}$ & {$\left[42^{\circ}, 72^{\circ}\right]$} & 2.2 & {$[1.2,3.1]$} & 23.13 & 95.87 & 294 & 28 & 5.5 & 74 \\
\hline
\end{tabular}


Table D.1 continued from previous page

\begin{tabular}{|c|c|c|c|c|c|c|c|c|c|c|c|c|c|}
\hline Station & Date & Phase & Grade & $\phi$ & $\phi$ error & $\delta t$ & $\delta t$ error & Lat & Lon & Baz & Depth & Mag & Energy reduction \\
\hline PEP & 16-Nov-2012 & SKKS & average & $18^{\circ}$ & {$\left[4^{\circ}, 36^{\circ}\right]$} & 2.1 & {$[1.2,3.2]$} & 49.28 & 155.43 & 347 & 29 & 6.5 & 71 \\
\hline PEP & 21-Jan-2013 & SKS & average & $-44^{\circ}$ & {$\left[-58^{\circ},-29^{\circ}\right]$} & 2.1 & {$[1.3,2.8]$} & 4.93 & 95.91 & 280 & 12 & 6.1 & 64 \\
\hline PEP & 30-Jan-2013 & SKS & average & $-25^{\circ}$ & {$\left[-45^{\circ}, 15^{\circ}\right]$} & 0.7 & {$[0.4,1.7]$} & -28.09 & -70.65 & 126 & 45 & 6.8 & 85 \\
\hline PEP & 31-Aug-2013 & SKS & average & $-34^{\circ}$ & {$\left[-45^{\circ},-25^{\circ}\right]$} & 3.8 & {$[2.9,4.0]$} & 51.24 & -174.82 & 6 & 10 & 5.2 & 97 \\
\hline PEP & 24-Sep-2013 & SKS & average & $51^{\circ}$ & {$\left[36^{\circ}, 64^{\circ}\right]$} & 3.5 & {$[2.7,4.0]$} & 7.37 & 59.54 & 259 & 4 & 5.5 & 58 \\
\hline PEP & 24-Sep-2013 & SKS & good & $49^{\circ}$ & {$\left[37^{\circ}, 62^{\circ}\right]$} & 2.8 & {$[2.1,3.6]$} & 25.38 & 65.97 & 280 & 10 & 5 & 96 \\
\hline PEP & 31-Oct-2013 & SKS & good & $7^{\circ}$ & {$\left[-4^{\circ}, 16^{\circ}\right]$} & 1.2 & {$[0.9,1.6]$} & -30.29 & -71.52 & 127 & 27 & 6.6 & 67 \\
\hline PEP & 03-Feb-2014 & SKKS & average & $71^{\circ}$ & {$\left[52^{\circ}, 82^{\circ}\right]$} & 2.4 & {$[1.3,3.6]$} & 38.26 & 20.39 & 276 & 5 & 6 & 90 \\
\hline PEP & 05-Feb-2014 & SKS & good & $77^{\circ}$ & {$\left[67^{\circ}, 87^{\circ}\right]$} & 1.0 & {$[0.8,1.1]$} & -21.38 & -69.52 & 122 & 40 & 5 & 72 \\
\hline SOW & 02-Oct-2013 & PKIKS & average & $19^{\circ}$ & {$\left[5^{\circ}, 36^{\circ}\right]$} & 2.5 & {$[1.2,3.7]$} & -15.06 & -71.92 & 116 & 108 & 5 & 79 \\
\hline sOW & 28-Oct-2013 & SKKS & average & $-38^{\circ}$ & {$\left[-55^{\circ},-25^{\circ}\right]$} & 2.1 & {$[1.2,3.0]$} & 56.61 & -34.30 & 38 & 6 & 5 & 90 \\
\hline WAI & 20-Feb-2013 & SKS & average & $-20^{\circ}$ & {$\left[-44^{\circ}, 10^{\circ}\right]$} & 0.9 & {$[0.4,1.7]$} & 10.60 & -41.04 & 122 & 10 & 5 & 77 \\
\hline WAI & 19-Apr-2013 & SKS & average & $53^{\circ}$ & {$\left[15^{\circ}, 66^{\circ}\right]$} & 1.7 & {$[0.5,3.4]$} & 46.22 & 150.79 & 343 & 110 & 7.2 & 88 \\
\hline WAI & 07-Nov-2012 & SKS & good & $34^{\circ}$ & {$\left[18^{\circ}, 50^{\circ}\right]$} & 1.7 & {$[1.2,2.3]$} & 13.99 & -91.90 & 81 & 24 & 7.4 & 85 \\
\hline WAI & 24-May-2013 & SKS & good & $40^{\circ}$ & {$\left[30^{\circ}, 49^{\circ}\right]$} & 1.9 & {$[1.5,2.4]$} & 54.89 & 153.22 & 347 & 598 & 8.3 & 97 \\
\hline WTA & 09-Apr-2013 & PKS & average & $45^{\circ}$ & {$\left[29^{\circ}, 61^{\circ}\right]$} & 1.7 & {$[1.1,2.5]$} & 28.43 & 51.59 & 274 & 12 & 6.4 & 88 \\
\hline WTA & 19-Apr-2013 & SKS & good & $52^{\circ}$ & {$\left[39^{\circ}, 60^{\circ}\right]$} & 1.8 & {$[1.1,2.6]$} & 46.22 & 150.79 & 344 & 110 & 7.2 & 96 \\
\hline WTA & 24-May-2013 & SKS & average & $46^{\circ}$ & {$\left[9^{\circ}, 64^{\circ}\right]$} & 1.0 & {$[0.5,2.1]$} & 52.24 & 151.44 & 346 & 624 & 6.7 & 90 \\
\hline WTA & 01-Oct-2013 & SKS & good & $39^{\circ}$ & {$\left[17^{\circ}, 56^{\circ}\right]$} & 1.5 & {$[1.0,2.3]$} & 53.20 & 152.79 & 347 & 573 & 6.7 & 90 \\
\hline
\end{tabular}




\begin{tabular}{|c|c|c|c|c|c|c|c|c|c|c|c|c|}
\hline Station & Date & Phase & Grade & $\phi$ & $\phi$ error & $\delta t$ & $\delta t$ error & Lat & Lon & Baz & Depth & Mag \\
\hline FUN & 08-Dec-2013 & SKS & good & $41^{\circ}$ & {$\left[21^{\circ}, 54^{\circ}\right]$} & $1.8 \mathrm{~s}$ & {$[1.1 \mathrm{~s}, 2.8 \mathrm{~s}]$} & 44.44 & 149.17 & 342 & 28 & 6 \\
\hline PAR & 08-Dec-2013 & SKS & average & $23^{\circ}$ & {$\left[-2^{\circ}, 51^{\circ}\right]$} & $1.3 \mathrm{~s}$ & {$[0.6 \mathrm{~s}, 2.3 \mathrm{~s}]$} & 44.44 & 149.17 & 342 & 28 & 6 \\
\hline $\mathrm{HOK}$ & 07-Nov-2012 & SKS & good & $45^{\circ}$ & {$\left[31^{\circ}, 56^{\circ}\right]$} & $2.3 \mathrm{~s}$ & {$[1.6 \mathrm{~s}, 3.0 \mathrm{~s}]$} & 13.99 & -91.90 & 81 & 24 & 7.4 \\
\hline KUR & 07-Nov-2012 & SKS & good & $14^{\circ}$ & {$\left[5^{\circ}, 30^{\circ}\right]$} & $2.7 \mathrm{~s}$ & {$[1.6 \mathrm{~s}, 3.9 \mathrm{~s}]$} & 13.99 & -91.90 & 81 & 24 & 7.4 \\
\hline MOT & 07-Nov-2012 & SKS & good & $46^{\circ}$ & {$\left[36^{\circ}, 56^{\circ}\right]$} & $3.4 \mathrm{~s}$ & {$[2.6 \mathrm{~s}, 4.0 \mathrm{~s}]$} & 13.99 & -91.90 & 81 & 24 & 7.4 \\
\hline PAR & 07-Nov-2012 & SKS & good & $28^{\circ}$ & {$\left[17^{\circ}, 41^{\circ}\right]$} & $1.7 \mathrm{~s}$ & {$[1.3 \mathrm{~s}, 2.3 \mathrm{~s}]$} & 13.99 & -91.90 & 81 & 24 & 7.4 \\
\hline PEP & 07-Nov-2012 & SKS & good & $28^{\circ}$ & {$\left[12^{\circ}, 49^{\circ}\right]$} & $2.2 \mathrm{~s}$ & {$[1.4 \mathrm{~s}, 3.1 \mathrm{~s}]$} & 13.99 & -91.90 & 81 & 24 & 7.4 \\
\hline WAI & 07-Nov-2012 & SKS & good & $34^{\circ}$ & {$\left[18^{\circ}, 50^{\circ}\right]$} & $1.7 \mathrm{~s}$ & {$[1.2 \mathrm{~s}, 2.3 \mathrm{~s}]$} & 13.99 & -91.90 & 81 & 24 & 7.4 \\
\hline $\mathrm{HOK}$ & 25-Mar-2013 & SKS & average & $12^{\circ}$ & {$\left[2^{\circ}, 24^{\circ}\right]$} & $3.5 \mathrm{~s}$ & {$[2.1 \mathrm{~s}, 4.0 \mathrm{~s}]$} & 14.49 & -90.46 & 82 & 189 & 6.2 \\
\hline $\mathrm{OIO}$ & 25-Mar-2013 & SKS & good & $42^{\circ}$ & {$\left[23^{\circ}, 59^{\circ}\right]$} & $2.3 \mathrm{~s}$ & {$[1.4 \mathrm{~s}, 3.2 \mathrm{~s}]$} & 14.49 & -90.46 & 82 & 189 & 6.2 \\
\hline PAR & 25-Mar-2013 & SKS & average & $29^{\circ}$ & {$\left[7^{\circ}, 58^{\circ}\right]$} & $1.5 \mathrm{~s}$ & {$[0.8 \mathrm{~s}, 3.0 \mathrm{~s}]$} & 14.49 & -90.46 & 82 & 189 & 6.2 \\
\hline HOK & 24-May-2013 & SKS & good & $40^{\circ}$ & {$\left[28^{\circ}, 51^{\circ}\right]$} & $1.5 \mathrm{~s}$ & {$[1.2 \mathrm{~s}, 1.9 \mathrm{~s}]$} & 54.89 & 153.22 & 347 & 598.1 & 8.3 \\
\hline PAR & 24-May-2013 & SKS & average & $54^{\circ}$ & {$\left[25^{\circ}, 67^{\circ}\right]$} & $1.7 \mathrm{~s}$ & {$[0.8 \mathrm{~s}, 3.3 \mathrm{~s}]$} & 54.89 & 153.22 & 347 & 598.1 & 8.3 \\
\hline WAI & 24-May-2013 & SKS & good & $40^{\circ}$ & {$\left[30^{\circ}, 49^{\circ}\right]$} & $1.9 \mathrm{~s}$ & {$[1.5 \mathrm{~s}, 2.4 \mathrm{~s}]$} & 54.89 & 153.22 & 347 & 598.1 & 8.3 \\
\hline PAR & 24-May-2013 & SKS & average & $35^{\circ}$ & {$\left[10^{\circ}, 56^{\circ}\right]$} & $1.5 \mathrm{~s}$ & {$[0.9 \mathrm{~s}, 2.5 \mathrm{~s}]$} & 52.24 & 151.44 & 346 & 624 & 6.7 \\
\hline WTA & 24-May-2013 & SKS & average & $46^{\circ}$ & {$\left[9^{\circ}, 64^{\circ}\right]$} & $1.0 \mathrm{~s}$ & {$[0.5 \mathrm{~s}, 2.1 \mathrm{~s}]$} & 52.24 & 151.44 & 346 & 624 & 6.7 \\
\hline HOK & 28-Sep-2013 & PKIKS & average & $57^{\circ}$ & {$\left[42^{\circ}, 71^{\circ}\right]$} & $2.7 \mathrm{~s}$ & {$[1.7 \mathrm{~s}, 3.7 \mathrm{~s}]$} & 27.18 & 65.51 & 281 & 12 & 6.8 \\
\hline PAR & 28-Sep-2013 & SKKS & average & $-41^{\circ}$ & {$\left[-65^{\circ},-14^{\circ}\right]$} & $1.5 \mathrm{~s}$ & {$[0.6 \mathrm{~s}, 2.5 \mathrm{~s}]$} & 27.18 & 65.51 & 281 & 12 & 6.8 \\
\hline
\end{tabular}


Table D.2 continued from previous page

\begin{tabular}{ccccccccccccc}
\hline Station & Date & Phase & Grade & $\phi$ & $\phi$ error & $\delta t$ & $\delta t$ error & Lat & Lon & Baz & Depth & Mag \\
\hline HOK & $01-$-Oct-2013 & SKS & average & $62^{\circ}$ & {$\left[45^{\circ}, 69^{\circ}\right]$} & $1.9 \mathrm{~s}$ & {$[0.8 \mathrm{~s}, 3.3 \mathrm{~s}]$} & 53.20 & 152.79 & 347 & 573 & 6.7 \\
OIO & $01-$-Oct-2013 & SKS & average & $64^{\circ}$ & {$\left[48^{\circ}, 71^{\circ}\right]$} & $2.1 \mathrm{~s}$ & {$[0.8 \mathrm{~s}, 3.6 \mathrm{~s}]$} & 53.20 & 152.79 & 347 & 573 & 6.7 \\
PAR & $01-$-Oct-2013 & SKS & good & $44^{\circ}$ & {$\left[21^{\circ}, 59^{\circ}\right]$} & $1.8 \mathrm{~s}$ & {$[1.1 \mathrm{~s}, 2.9 \mathrm{~s}]$} & 53.20 & 152.79 & 347 & 573 & 6.7 \\
WTA & $01-$-Oct-2013 & SKS & good & $39^{\circ}$ & {$\left[17^{\circ}, 56^{\circ}\right]$} & $1.5 \mathrm{~s}$ & {$[1.0 \mathrm{~s}, 2.3 \mathrm{~s}]$} & 53.20 & 152.79 & 347 & 573 & 6.7 \\
\hline KUR & $19-A p r-2013$ & SKS & average & $57^{\circ}$ & {$\left[51^{\circ}, 62^{\circ}\right]$} & $3.8 \mathrm{~s}$ & {$[2.7 \mathrm{~s}, 4.0 \mathrm{~s}]$} & 46.22 & 150.79 & 343 & 110 & 7.2 \\
MOT & $19-A p r-2013$ & SKS & average & $57^{\circ}$ & {$\left[22^{\circ}, 66^{\circ}\right]$} & $1.1 \mathrm{~s}$ & {$[0.4 \mathrm{~s}, 2.2 \mathrm{~s}]$} & 46.22 & 150.79 & 343 & 110 & 7.2 \\
PAR & $19-A p r-2013$ & SKS & good & $47^{\circ}$ & {$\left[31^{\circ}, 58^{\circ}\right]$} & $2.1 \mathrm{~s}$ & {$[1.2 \mathrm{~s}, 3.0 \mathrm{~s}]$} & 46.22 & 150.79 & 343 & 110 & 7.2 \\
WAI & $19-A p r-2013$ & SKS & average & $53^{\circ}$ & {$\left[15^{\circ}, 66^{\circ}\right]$} & $1.7 \mathrm{~s}$ & {$[0.5 \mathrm{~s}, 3.4 \mathrm{~s}]$} & 46.22 & 150.79 & 343 & 110 & 7.2 \\
WTA & $19-A p r-2013$ & SKS & good & $52^{\circ}$ & {$\left[39^{\circ}, 60^{\circ}\right]$} & $1.8 \mathrm{~s}$ & {$[1.1 \mathrm{~s}, 2.6 \mathrm{~s}]$} & 46.22 & 150.79 & 344 & 110 & 7.2 \\
\hline PAR & $16-N o v-2012$ & SKS & average & $46^{\circ}$ & {$\left[31^{\circ}, 58^{\circ}\right]$} & $2.5 \mathrm{~s}$ & {$[1.6 \mathrm{~s}, 3.5 \mathrm{~s}]$} & 49.28 & 155.43 & 347 & 29 & 6.5 \\
PEP & $16-N o v-2012$ & SKKS & average & $18^{\circ}$ & {$\left[4^{\circ}, 36^{\circ}\right]$} & $2.1 \mathrm{~s}$ & {$[1.2 \mathrm{~s}, 3.2 \mathrm{~s}]$} & 49.28 & 155.43 & 347 & 29 & 6.5 \\
\hline
\end{tabular}




\section{Bibliography}

Allen, R. V. (1978). Automatic earthquake recognition and timing from single traces. Bulletin of the Seismological Society of America, 68(5):1521-1532.

Altamimi, Z., Métivier, L., and Collilieux, X. (2012). ITRF2008 plate motion model. Journal of Geophysical Research: Solid Earth, 117(B7).

Altman, D. G. M., David, T. N., and Bryant, M. J. (2000). Gardner. Statistics with confidence.

Anderson, E. M. (1905). The dynamics of faulting. Transactions of the Edinburgh Geological Society, 8(3):387-402.

Ando, M., Ishikawa, Y., and Yamazaki, F. (1983). Shear wave polarization anisotropy in the upper mantle beneath Honshu, Japan. Journal of Geophysical Research: Solid Earth, 88(B7):5850-5864.

Audet, P., Bostock, M. G., Boyarko, D. C., Brudzinski, M. R., and Allen, R. M. (2010). Slab morphology in the Cascadia fore arc and its relation to episodic tremor and slip. Journal of Geophysical Research: Solid Earth, 115(B4).

Audoine, E., Savage, M. K., and Gledhill, K. (2000). Seismic anisotropy from local earthquakes in the transition region from a subduction to a strike-slip plate boundary, New Zealand. Journal of Geophysical Research: Solid Earth, 105(B4):8013-8033.

Audoine, E., Savage, M. K., and Gledhill, K. (2004). Anisotropic structure under a back arc spreading region, the Taupo Volcanic Zone, New Zealand. Journal of Geophysical Research: Solid Earth, 109(B11).

Baba, T., Hori, T., Hirano, S., Cummins, P. R., Park, J.-O., Kameyama, M., and Kaneda, Y. (2001). Deformation of a seamount subducting beneath an accre- 
tionary prism: Constraints from numerical simulation. Geophysical Research Letters, 28(9):1827-1830.

Babuska, V. and Cara, M. (1991). Seismic anisotropy in the Earth, volume 10. Springer Science \& Business Media.

Balfour, N. J., Savage, M. K., and Townend, J. (2005). Stress and crustal anisotropy in Marlborough, New Zealand: evidence for low fault strength and structurecontrolled anisotropy. Geophysical Journal International, 163(3):1073-1086.

Barker, D. H. N., Henrys, S., Caratori Tontini, F., Barnes, P. M., Bassett, D., Todd, E., and Wallace, L. (2018). Geophysical constraints on the relationship between seamount subduction, slow slip, and tremor at the north Hikurangi subduction zone, New Zealand. Geophysical Research Letters, 45(23):804-812.

Barker, D. H. N., Sutherland, R., Henrys, S., and Bannister, S. (2009). Geometry of the Hikurangi subduction thrust and upper plate, North Island, New Zealand. Geochemistry, Geophysics, Geosystems, 10(2).

Bassett, D., Sutherland, R., and Henrys, S. (2014). Slow wavespeeds and fluid overpressure in a region of shallow geodetic locking and slow slip, Hikurangi subduction margin, New Zealand. Earth and Planetary Science Letters, 389(2):113.

Beanland, S. and Haines, J. (1998). The kinematics of active deformation in the North Island, New Zealand, determined from geological strain rates. New Zealand Journal of Geology and Geophysics, 41(4):311-323.

Beavan, J., Ellis, S., Wallace, L., and Denys, P. (2007). Kinematic constraints from GPS on oblique convergence of the Pacific and Australian Plates, central South Island, New Zealand. Washington DC American Geophysical Union Geophysical Monograph Series, 175:75-94.

Beavan, J., Tregoning, P., Bevis, M., Kato, T., and Meertens, C. (2002). Motion and rigidity of the Pacific Plate and implications for plate boundary deformation. Journal of Geophysical Research: Solid Earth, 107(B10):ETG-19.

Beavan, J., Wallace, L. M., Palmer, N., Denys, P., Ellis, S., Fournier, N., Hreinsdottir, S., Pearson, C., and Denham, M. (2016). New Zealand GPS velocity field: 19952013. New Zealand Journal of Geology and Geophysics, 59(1):5-14. 
Bell, R., Sutherland, R., Barker, D. H. N., Henrys, S., Bannister, S., Wallace, L., and Beavan, J. (2010). Seismic reflection character of the Hikurangi subduction interface, New Zealand, in the region of repeated Gisborne slow slip events. Geophysical Journal International, 180(1):34-48.

Boness, N. L. and Zoback, M. D. (2006). Mapping stress and structurally controlled crustal shear velocity anisotropy in California. Geology, 34(10):825-828.

Carter, R. M. and Naish, T. R. (1998). A review of Wanganui Basin, New Zealand: global reference section for shallow marine, Plio-Pleistocene (2.5-0 Ma) cyclostratigraphy. Sedimentary Geology, 122(1):37-52.

Castellazzi, C., Savage, M. K., Walsh, E., and Arnold, R. (2015). Shear wave automatic picking and splitting measurements at Ruapehu volcano, New Zealand. Journal of Geophysical Research: Solid Earth, 120(5):3363-3384.

Cichowicz, A. (1993). An automatic S-phase picker. Bulletin of the Seismological Society of America, 83(1):180-189.

Cochran, E. S. (1999). SKS shear-wave splitting observations in the mantle beneath East Cape, New Zealand. University of Leeds.

Cochran, E. S., Li, Y.-G., and Vidale, J. E. (2006). Anisotropy in the shallow crust observed around the San Andreas Fault before and after the 2004 M 6.0 Parkfield Earthquake. Bulletin of the Seismological Society of America, 96(4B):364-375.

Coffin, M. F., Gahagan, L. M., and Lawver, L. A. (1998). Present-day plate boundary digital data compilation. University of Texas Institute for geophysics technical report, 174(5).

Crampin, S. (1981). A review of wave motion in anisotropic and cracked elasticmedia. Wave Motion, 3(4):343-391.

Crampin, S. and Chastin, S. (2003). A review of shear wave splitting in the crackcritical crust. Geophysical Journal International, 155(1):221-240.

Crotwell, H. P., Owens, T. J., and Ritsema, J. (1999). The TauP Toolkit: Flexible seismic travel-time and ray-path utilities. Seismological Research Letters, 70(2):154. 
DeMets, C., Gordon, R. G., Argus, D. F., and Stein, S. (1994). Effect of recent revisions to the geomagnetic reversal time scale on estimates of current plate motions. Geophysical Research Letters, 21(20):2191-2194.

Diehl, T., Deichmann, N., Kissling, E., and Husen, S. (2009). Automatic S-wave picker for local earthquake tomography. Bulletin of the Seismological Society of America, 99(3):1906-1920.

Dimech, J.-L. (2016). Seismic investigations of the lithosphere in an amagmatic back-arc region: North Island, New Zealand. PhD thesis, Victoria University of Wellington, Wellington, New Zealand.

Dimech, J.-L., Stern, T., and Lamb, S. (2017). Mantle earthquakes, crustal structure, and gravitational instability beneath western North Island, New Zealand. Geology, 45(2):155-158.

Dominguez, S., Lallemand, S. E., Malavieille, J., and von Huene, R. (1998). Upper plate deformation associated with seamount subduction. Tectonophysics, 293(3):207-224.

Doser, D. I. and Webb, T. H. (2003). Source parameters of large historical (19171961) earthquakes, North Island, New Zealand. Geophysical Journal International, 152(3):795-832.

Douglas, A., Beavan, J., Wallace, L., and Townend, J. (2005). Slow slip on the northern Hikurangi subduction interface, New Zealand. Geophysical Research Letters, 32(16).

Downes, G. L. and Grapes, R. H. (1999). The 1855 Wairarapa, New Zealand, earthquake: historical data. Institute of Geological \& Nuclear Sciences Ltd.

Eberhart-Phillips, D., Bannister, S., and Reyners, M. (2017). Deciphering the 3-D distribution of fluid along the shallow Hikurangi subduction zone using P-and S-wave attenuation. Geophysical Journal International, 211(2):1054-1067.

Eberhart-Phillips, D. and Reyners, M. (2009). Three-dimensional distribution of seismic anisotropy in the Hikurangi subduction zone beneath the central North Island, New Zealand. Journal of Geophysical Research: Solid Earth, 114(B6). 
Eberhart-Phillips, D., Reyners, M., Bannister, S., Chadwick, M., and Ellis, S. (2010). Establishing a versatile 3-D seismic velocity model for New Zealand. Seismological Research Letters, 81(6):992-1000.

Eberhart-Phillips, D., Reyners, M., Chadwick, M., and Stuart, G. (2008). Threedimensional attenuation structure of the Hikurangi subduction zone in the central North Island, New Zealand. Geophysical Journal International, 174(1):418-434.

Ellis, S., Fagereng, A., Barker, D., Henrys, S., Saffer, D., Wallace, L., Williams, C., and Harris, R. (2015). Fluid budgets along the northern Hikurangi subduction margin, New Zealand: the effect of a subducting seamount on fluid pressure. Geophysical Journal International, 202(1):277-297.

Evanzia, D., Wilson, T., Savage, M. K., Lamb, S., and Hirschberg, H. (2017). Stress orientations in a locked subduction one at the southern Hikurangi Margin, New Zealand. Journal of Geophysical Research: Solid Earth, 122(10):7895-7911.

Flesch, L. M., Haines, A. J., and Holt, W. E. (2001). Dynamics of the India-Eurasia collision zone. Journal of Geophysical Research: Solid Earth, 106(B8):16435-16460.

Flesch, L. M., Holt, W. E., Haines, A. J., Wen, L., and Shen-Tu, B. (2007). The dynamics of western North America: stress magnitudes and the relative role of gravitational potential energy, plate interaction at the boundary and basal tractions. Geophysical Journal International, 169(3):866-896.

Fouch, M. J. and Fischer, K. M. (1998). Shear wave anisotropy in the Mariana Subduction Zone. Geophysical Research Letters, 25(8):1221-1224.

Gao, Y. and Crampin, S. (2006). A stress-forecast earthquake (with hindsight), where migration of source earthquakes causes anomalies in shear-wave polarisations. Tectonophysics, 426(3):253-262.

Gerst, A. and Savage, M. K. (2004). Seismic anisotropy beneath Ruapehu Volcano: A possible eruption forecasting tool. Science, 306(5701):1543-1547.

Gledhill, K. and Stuart, G. (1996). Seismic anisotropy in the fore-arc region of the Hikurangi subduction zone, New Zealand. Physics of the Earth and Planetary Interiors, 95(3):211-225. 
Gledhill, K. R. (1993). Shear waves recorded on close-spaced seismographs: I. Shear-wave splitting results. Can. J. Expl. Geophys, 29(1):285-298.

Greve, S. M., Savage, M. K., and Hofmann, S. D. (2008). Strong variations in seismic anisotropy across the Hikurangi subduction zone, North Island, New Zealand. Tectonophysics, 462(1):7-21.

Harmsen, F. R. (1985). Lithostratigraphy of Pliocene strata, Central and Southern Hawke's Bay, New Zealand. New Zealand Journal of Geology and Geophysics, 28(3):413-433.

Hatherton, T. (1970). Upper mantle inhomogeneity beneath New Zealand: Surface manifestations. Journal of Geophysical Research (1896-1977), 75(2):269-284.

Head, T. J. (2001). The nature of shallow anisotropy in the southern Raukumara Peninsula, New Zealand, determined by shear-wave splitting measurements from local earthquakes. PhD thesis, University of Leeds, Leeds, UK.

Hirschberg, H., Savage, M., and Lamb, S. (2017). Calculation of the Deviatoric Stress Field in New Zealand. Victoria University of Wellington, Wellington, New Zealand, MSc Thesis.

Hirschberg, H. P., Lamb, S., and Savage, M. K. (2019). Strength of an obliquely convergent plate boundary: lithospheric stress magnitudes and viscosity in New Zealand. Geophysical Journal International, 216(2):1005-1024.

Holt, R. (2013). Seismic anisotropy and stress of the canterbury plains. PhD thesis, Victoria University of Wellington, Wellington, New Zealand.

Husen, S. and Kissling, E. (2001). Postseismic fluid flow after the large subduction earthquake of Antofagasta, Chile. Geology, 29(9):847-850.

Illsley-Kemp, F., Savage, M. K., Wilson, C. J. N., and Bannister, S. (2019). Mapping stress and structure from subducting slab to magmatic rift: crustal seismic anisotropy of the North Island, New Zealand. Geochemistry, Geophysics, Geosystems, 20(11):5038-5056.

Jeanloz, R., Knittle, E., O\&apos;Nions, R. K., Clayton, R., and Parsons, B. (1989). Density and composition of the lower mantle. Philosophical Transactions of the 
Royal Society of London. Series A, Mathematical and Physical Sciences, 328(1599):377389.

Ji, S. and Salisbury, M. H. (1993). Shear-wave velocities, anisotropy and splitting in high-grade mylonites. Tectonophysics, 221(3):453-473.

Johnson, J. H., Savage, M. K., and Townend, J. (2011). Distinguishing between stress-induced and structural anisotropy at Mount Ruapehu volcano, New Zealand. Journal of Geophysical Research: Solid Earth, 116(B12).

Jung, H. and Karato, S.-I. (2001a). Effects of water on dynamically recrystallized grain-size of olivine. Journal of Structural Geology, 23(9):1337-1344.

Jung, H. and Karato, S.-i. (2001b). Water-induced fabric transitions in olivine. Science, 293(5534):1460-1463.

Kaneko, Y., Ito, Y., Chow, B., Wallace, L. M., Tape, C., Grapenthin, R., D'Anastasio, E., Henrys, S., and Hino, R. (2019). Ultra-long duration of seismic ground motion arising from a thick, low velocity sedimentary wedge. Journal of Geophysical Research: Solid Earth.

Karalliyadda, S. C. and Savage, M. K. (2013). Seismic anisotropy and lithospheric deformation of the plate-boundary zone in South Island, New Zealand: inferences from local S-wave splitting. Geophysical Journal International, 193(2):507530.

Karato, S.-i., Jung, H., Katayama, I., and Skemer, P. (2008). Geodynamic significance of seismic anisotropy of the upper mantle: New insights from laboratory studies. Annual Review of Earth and Planetary Sciences, 36(1):59-95.

Katayama, I., Jung, H., and Karato, S.-i. (2004). New type of olivine fabric from deformation experiments at modest water content and low stress. Geology, 32(12):1045-1048.

Kendall, J.-M. (2013). Seismic anisotropy in the boundary layers of the mantle. In Earth's Deep Interior: Mineral Physics and Tomography From the Atomic to the Global Scale, pages 133-159. American Geophysical Union (AGU).

Kisslinger, C. and Engdahl, E. R. (1973). The interpretation of the Wadati diagram with relaxed assumptions. Bulletin of the Seismological Society of America, 63(5):1723-1736. 
Kudo, T. and Yamaoka, K. (2003). Pull-down basin in the central part of Japan due to subduction-induced mantle flow. Tectonophysics, 367(3):203-217.

Lamb, S. (2015). Kinematics to dynamics in the New Zealand Plate boundary zone: implications for the strength of the lithosphere. Geophysical Journal International, 201(2):552-573.

Lamb, S. and Smith, E. (2013). The nature of the plate interface and driving force of interseismic deformation in the New Zealand plate-boundary zone, revealed by the continuous GPS velocity field. Journal of Geophysical Research: Solid Earth, 118(6):3160-3189.

Langridge, R. M., Ries, W. F., Litchfield, N. J., Villamor, P., Dissen, R. J. V., Barrell, D. J. A., Rattenbury, M. S., Heron, D. W., Haubrock, S., Townsend, D. B., Lee, J. M., Berryman, K. R., Nicol, A., Cox, S. C., and Stirling, M. W. (2016). The New Zealand active faults database. New Zealand Journal of Geology and Geophysics, 59(1):86-96.

Leonard, M. and Kennett, B. L. N. (1999). Multi-component autoregressive techniques for the analysis of seismograms. Physics of the Earth and Planetary Interiors, 113(1-4):247-263.

Li, Z. and Peng, Z. (2017). Stress- and structure-induced anisotropy in southern California from two decades of shear wave splitting measurements. Geophysical Research Letters, 44(19):9607-9614.

Litchfield, N. J., Van Dissen, R., Sutherland, R., Barnes, P. M., Cox, S. C., Norris, R., Beavan, R. J., Langridge, R., Villamor, P., Berryman, K., and others (2014). A model of active faulting in New Zealand. New Zealand Journal of Geology and Geophysics, 57(1):32-56.

Lodewyk, J. A. and Evers, B. (2014). OBSIP instrumentation and operations for the Cascadia Initiative. AGU Fall Meeting Abstracts, pages S13B-4442.

Long, M. D. and Silver, P. G. (2009). Shear wave splitting and mantle anisotropy: Measurements, interpretations, and new directions. Surveys in Geophysics, 30(4):407-461.

Love, A. E. H. (2013). A treatise on the mathematical theory of elasticity. Cambridge university press. 
Lucente, F. P., De Gori, P., Margheriti, L., Piccinini, D., Di Bona, M., Chiarabba, C., and Piana Agostinetti, N. (2010). Temporal variation of seismic velocity and anisotropy before the 2009 MW 6.3 L'Aquila earthquake, Italy. Geology, 38(11):1015-1018.

Marson-Pidgeon, K. and Savage, M. K. (1997). Frequency-dependent anisotropy in Wellington, New Zealand. Geophysical Research Letters, 24(24):3297-3300.

Marson-Pidgeon, K. and Savage, M. K. (2004). Shear-wave splitting variations across an array in the southern North Island, New Zealand. Geophysical Research Letters, 31(21).

Marson-Pidgeon, K., Savage, M. K., Gledhill, K., and Stuart, G. (1999). Seismic anisotropy beneath the lower half of the North Island, New Zealand. Journal of Geophysical Research: Solid Earth, 104(B9):20277-20286.

McCubbine, J. C., Stagpoole, V., Tontini, F. C., Amos, M., Smith, E., and Winefield, R. (2017). Gravity anomaly grids for the New Zealand region. New Zealand Journal of Geology and Geophysics, 60(4):381-391.

Miller, V. and Savage, M. (2001). Changes in seismic anisotropy after volcanic eruptions: Evidence from Mount Ruapehu. Science, 293(5538):2231-2233.

Mooney, H. M. (1970). Upper mantle inhomogeneity beneath New Zealand: Seismic evidence. Journal of Geophysical Research (1896-1977), 75(2):285-309.

Morley, A. M., Stuart, G. W., Kendall, J.-M., and Reyners, M. (2006). Mantle wedge anisotropy in the Hikurangi subduction zone, central North Island, New Zealand. Geophysical Research Letters, 33(5).

Mortimer, N. (2004). New Zealand's geological foundations. Gondwana Research, $7(1): 261-272$.

Mroczek, S., Savage, M. K., Hopp, C., and Sewell, S. M. (2019). Anisotropy as an indicator for reservoir changes: example from the Rotokawa and Ngatamariki geothermal fields, New Zealand. Geophysical Journal International, 220(1):1-17.

Nakajima, J. and Hasegawa, A. (2004). Shear-wave polarization anisotropy and subduction-induced flow in the mantle wedge of northeastern Japan. Earth and Planetary Science Letters, 225(3):365-377. 
Nakajima, J. and Uchida, N. (2018). Repeated drainage from megathrusts during episodic slow slip. Nature Geoscience, 11(5):351.

Nur, A. (1971). Effects of stress on velocity anisotropy in rocks with cracks. Journal of Geophysical Research, 76(8):2022-2034.

Nur, A. and Simmons, G. (1969). Stress-induced velocity anisotropy in rock: An experimental study. Journal of Geophysical Research (1896-1977), 74(27):6667-6674.

Nuttli, O. (1961). The effect of the earth's surface on the $S$ wave particle motion. Bulletin of the Seismological Society of America, 51(2):237.

Obara, K. and Kato, A. (2016). Connecting slow earthquakes to huge earthquakes. Science, 353(6296):253-257.

Park, J. and Levin, V. (2002). Seismic anisotropy: Tracing plate dynamics in the mantle. Science, 296(5567):485-489.

Peng, Z. and Ben-Zion, Y. (2004). Systematic analysis of crustal anisotropy along the Karadere-Düzce branch of the North Anatolian fault. Geophysical Journal International, 159(1):253-274.

Peng, Z. and Gomberg, J. (2010). An integrated perspective of the continuum between earthquakes and slow-slip phenomena. Nature Geoscience, 3:599.

Reiss, M. C. and Rümpker, G. (2017). SplitRacer: MATLAB code and GUI for semiautomated analysis and interpretation of teleseismic shear-wave splitting. Seismological Research Letters, 88(2A):392-409.

Reyners, M., Eberhart-Phillips, D., and Bannister, S. (2011). Tracking repeated subduction of the Hikurangi Plateau beneath New Zealand. Earth and Planetary Science Letters, 311(1):165-171.

Reyners, M., Eberhart-Phillips, D., Stuart, G., and Nishimura, Y. (2006). Imaging subduction from the trench to $300 \mathrm{~km}$ depth beneath the central North Island, New Zealand, with Vp and Vp/Vs. Geophysical Journal International, 165(2):565583.

Reyners, M. and Stuart, G. (2002). The central North Island passive seismic experiment. Institute of Geological \& Nuclear Sciences. 
Ribe, N. M. (1989). Seismic anisotropy and mantle flow. Journal of Geophysical Research: Solid Earth, 94(B4):4213-4223.

Rowland, J. V. and Sibson, R. H. (2001). Extensional fault kinematics within the Taupo Volcanic Zone, New Zealand: Soft-linked segmentation of a continental rift system. New Zealand Journal of Geology and Geophysics, 44(2):271-283.

Rumpker, G. and Silver, P. G. (1998). Apparent shear-wave splitting parameters in the presence of vertically varying anisotropy. Geophysical Journal International, 135(3):790-800.

Salmon, M. L., Stern, T. A., and Savage, M. K. (2011). A major step in the continental Moho and its geodynamic consequences: the Taranaki-Ruapehu line, New Zealand. Geophysical Journal International, 186(1):32-44.

Savage, M. K., Ohminato, T., Aoki, Y., Tsuji, H., and Greve, S. M. (2010a). Stress magnitude and its temporal variation at Mt. Asama Volcano, Japan, from seismic anisotropy and GPS. Earth and Planetary Science Letters, 290(3):403-414.

Savage, M. K. and Silver, P. G. (1993). Mantle deformation and tectonics: constraints from seismic anisotropy in the western United States. Physics of the Earth and Planetary Interiors, 78(3-4):207-227.

Savage, M. K., Wessel, A., Teanby, N. A., and Hurst, A. W. (2010b). Automatic measurement of shear wave splitting and applications to time varying anisotropy at Mount Ruapehu volcano, New Zealand. Journal of Geophysical Research: Solid Earth, 115(B12).

Savage, M. S. (1999). Seismic anisotropy and mantle deformation: What have we learned from shear wave splitting? Reviews of Geophysics, 37(1):65-106.

Schwartz, S. Y. and Rokosky, J. M. (2007). Slow slip events and seismic tremor at circum-Pacific subduction zones. Reviews of Geophysics, 45(3).

Seward, A. M., Henderson, C. M., and Smith, E. G. C. (2009). Models of the upper mantle beneath the central North Island, New Zealand, from speeds and anisotropy of subhorizontal P waves (Pn). Journal of Geophysical Research: Solid Earth, 114(B1). 
Shaddox, H. R. and Schwartz, S. Y. (2019). Subducted seamount diverts shallow slow slip to the forearc of the northern Hikurangi subduction zone, New Zealand. Geology, 47(5):415-418.

Sierd Cloetingh, H. K. and Groenewoud, W. (1989). Intraplate stresses and sedimentary basin evolution. Geophysical Monograph Series.

Silver, P. G. and Chan, W. W. (1988). Implications for continental structure and evolution from seismic anisotropy. 335(6185).

Silver, P. G. and Chan, W. W. (1991). Shear wave splitting and subcontinental mantle deformation. Journal of Geophysical Research: Solid Earth, 96(B10):1642916454.

Stachnik, J. C., Sheehan, A. F., Zietlow, D. W., Yang, Z., Collins, J., and Ferris, A. (2012). Determination of New Zealand ocean bottom seismometer orientation via Rayleigh-wave polarization. Seismological Research Letters, 83(4):704.

Stein, S. and Wysession, M. (2003). An introduction to seismology, earthquakes, and earth structure. John Wiley \& Sons.

Stern, T. and Benson, A. (2011). Wide-angle seismic imaging beneath an andesitic arc: Central North Island, New Zealand. Journal of Geophysical Research: Solid Earth, 116(B9).

Stern, T., Houseman, G., Salmon, M., and Evans, L. (2013). Instability of a lithospheric step beneath western North Island, New Zealand. Geology, 41(4):423426.

Stern, T., Smith, E. G. C., Davey, F. J., and Muirhead, K. J. (1987). Crustal and upper mantle structure of the northwestern North Island, New Zealand, from seismic refraction data. Geophysical Journal International, 91(3):913-936.

Stern, T. A., Quinlan, G. M., and Holt, W. E. (1992). Basin formation behind an active subduction zone: three-dimensional flexural modelling of Wanganui Basin, New Zealand. Basin Research, 4(3-4):197-214.

Stern, T. A., Stratford, W. R., and Salmon, M. L. (2006). Subduction evolution and mantle dynamics at a continental margin: Central North Island, New Zealand. Reviews of Geophysics, 44(4). 
Stratford, W. R. and Stern, T. A. (2006). Crust and upper mantle structure of a continental backarc: central North Island, New Zealand. Geophysical Journal International, 166(1):469-484.

Teanby, N., Kendall, J.-M., Jones, R. H., and Barkved, O. (2004a). Stress-induced temporal variations in seismic anisotropy observed in microseismic data. Geophysical Journal International, 156(3):459-466.

Teanby, N. A., Kendall, J.-M., and van der Baan, M. (2004b). Automation of shearwave splitting measurements using cluster analysis. Bulletin of the Seismological Society of America, 94(2):453.

Todd, E. K., Schwartz, S. Y., Mochizuki, K., Wallace, L. M., Sheehan, A. F., Webb, S. C., Williams, C. A., Nakai, J., Yarce, J., Fry, B., Henrys, S., and Ito, Y. (2018). Earthquakes and tremor linked to seamount subduction during shallow slow slip at the Hikurangi Margin, New Zealand. Journal of Geophysical Research: Solid Earth, 123(8):6769-6783.

Townend, J., Sherburn, S., Arnold, R., Boese, C., and Woods, L. (2012). Threedimensional variations in present-day tectonic stress along the Australia-Pacific plate boundary in New Zealand. Earth and Planetary Science Letters, 353-354:4759.

Townend, J. and Zoback, M. D. (2000). How faulting keeps the crust strong. Geology, 28(5):399-402.

Tozer, B., Stern, T. A., Lamb, S. L., and Henrys, S. A. (2017). Crust and uppermantle structure of Wanganui Basin and southern Hikurangi margin, North Island, New Zealand as revealed by active source seismic data. Geophysical Journal International, 211(2):718-740.

Uchida, N., Iinuma, T., Nadeau, R. M., Bürgmann, R., and Hino, R. (2016). Periodic slow slip triggers megathrust zone earthquakes in northeastern Japan. Science, 351(6272):488-492.

Unglert, K. (2011). Crustal cracks in areas of active deformation: Correlation of GPS and seismic anisotropy. PhD thesis, Victoria University of Wellington, NZ. 
Villamor, P. and Berryman, K. R. (2006). Late Quaternary geometry and kinematics of faults at the southern termination of the Taupo Volcanic Zone, New Zealand. New Zealand Journal of Geology and Geophysics, 49(1):1-21.

Wadati, K. and Oki, S. (1933). On the travel time of earthquake waves.(Part II). Journal of the Meteorological Society of Japan. Ser. II, 11(1):14-28.

Walcott, R. I. (1984). The kinematics of the plate boundary zone through New Zealand: a comparison of short- and long-term deformations. Geophysical Journal International, 79(2):613-633.

Wallace, L. M., Barnes, P., Beavan, J., Van Dissen, R., Litchfield, N., Mountjoy, J., Langridge, R., Lamarche, G., and Pondard, N. (2012a). The kinematics of a transition from subduction to strike-slip: An example from the central New Zealand plate boundary. Journal of Geophysical Research: Solid Earth, 117(B2).

Wallace, L. M., Bartlow, N., Hamling, I., and Fry, B. (2014). Quake clamps down on slow slip. Geophysical Research Letters, 41(24):8840-8846.

Wallace, L. M. and Beavan, J. (2006). A large slow slip event on the central Hikurangi subduction interface beneath the Manawatu region, North Island, New Zealand. Geophysical Research Letters, 33(11).

Wallace, L. M. and Beavan, J. (2010). Diverse slow slip behavior at the Hikurangi subduction margin, New Zealand. Journal of Geophysical Research: Solid Earth, 115(B12).

Wallace, L. M., Beavan, J., Bannister, S., and Williams, C. (2012b). Simultaneous long-term and short-term slow slip events at the Hikurangi subduction margin, New Zealand: Implications for processes that control slow slip event occurrence, duration, and migration. Journal of Geophysical Research: Solid Earth, 117(B11).

Wallace, L. M., Beavan, J., McCaffrey, R., and Darby, D. (2004). Subduction zone coupling and tectonic block rotations in the North Island, New Zealand. Journal of Geophysical Research: Solid Earth, 109(B12).

Wallace, L. M. and Eberhart-Phillips, D. (2013). Newly observed, deep slow slip events at the central Hikurangi margin, New Zealand: Implications for downdip variability of slow slip and tremor, and relationship to seismic structure. Geophysical Research Letters, 40(20):5393-5398. 
Wallace, L. M., Hreinsdottir, S., Ellis, S., Hamling, I., D'Anastasio, E., and Denys, P. (2018). Triggered slow slip and afterslip on the southern Hikurangi Subduction Zone following the Kaikoura Earthquake. Geophysical Research Letters, 45(10):4710-4718.

Wallace, L. M., Reyners, M., Cochran, U., Bannister, S., Barnes, P. M., Berryman, K., Downes, G., Eberhart-Phillips, D., Fagereng, A., Ellis, S., Nicol, A., McCaffrey, R., Beavan, R. J., Henrys, S., Sutherland, R., Barker, D. H. N., Litchfield, N., Townend, J., Robinson, R., Bell, R., Wilson, K., and Power, W. (2009). Characterizing the seismogenic zone of a major plate boundary subduction thrust: Hikurangi Margin, New Zealand. Geochemistry, Geophysics, Geosystems, 10(10).

Wallace, L. M., Webb, S. C., Ito, Y., Mochizuki, K., Hino, R., Henrys, S., Schwartz, S. Y., and Sheehan, A. F. (2016). Slow slip near the trench at the Hikurangi subduction zone, New Zealand. Science, 352(6286):701-704.

Wang, X.-Q., Schubnel, A., Fortin, J., David, E. C., Guéguen, Y., and Ge, H.-K. (2012). High Vp/Vs ratio: Saturated cracks or anisotropy effects? Geophysical Research Letters, 39(11).

Warren-Smith, E., Fry, B., Wallace, L., Chon, E., Henrys, S. A., Sheehan, A. F., Mochizuki, K., Schwartz, S. Y., and Lebedev, S. (2019). Episodic stress and fluid pressure cycling in subducting oceanic crust during slow slip. Nature Geoscience, 12:475-481.

Webb, S., Gaw, S., Marsden, I. D., and McRae, N. K. (2020). Biomarker responses in New Zealand green-lipped mussels Perna canaliculus exposed to microplastics and triclosan. Ecotoxicology and Environmental Safety, 201:110871.

Wessel, P., Smith, W. H. F., Scharroo, R., Luis, J., and Wobbe, F. (2013). Generic mapping tools: improved version released. Eos, Transactions American Geophysical Union, 94(45):409-410.

Williams, C. A., Eberhart-Phillips, D., Bannister, S., Barker, D. H. N., Henrys, S., Reyners, M., and Sutherland, R. (2013). Revised interface geometry for the Hikurangi Subduction Zone, New Zealand. Seismological Research Letters, 84(6):1066.

Wilson, C. J. N., Houghton, B. F., McWilliams, M. O., Lanphere, M. A., Weaver, S. D., and Briggs, R. M. (1995). Volcanic and structural evolution of Taupo Vol- 
canic Zone, New Zealand: a review. Journal of Volcanology and Geothermal Research, 68(1):1-28.

Wolfe, C. J. and Silver, P. G. (1998). Seismic anisotropy of oceanic upper mantle: Shear wave splitting methodologies and observations. Journal of Geophysical Research: Solid Earth, 103(B1):749-771.

Wood, R. and Davy, B. (1994). The Hikurangi Plateau. Marine Geology, 118(1):153173.

Yarce, J., Sheehan, A. F., Nakai, J. S., Schwartz, S. Y., Mochizuki, K., Savage, M. K., Wallace, L. M., Henrys, S. A., Webb, S. C., Ito, Y., Abercrombie, R. E., Fry, B., Shaddox, H., and Todd, E. K. (2019). Seismicity at the northern Hikurangi Margin, New Zealand, and investigation of the potential spatial and temporal relationships with a shallow slow slip event. Journal of Geophysical Research: Solid Earth, 124(5):4751-4766.

Zal, H. J., Jacobs, K., Savage, M. K., Yarce, J., Mroczek, S., Graham, K., Todd, E. K., Nakai, J., Iwasaki, Y., Sheehan, A., Mochizuki, K., Wallace, L., Schwartz, S., Webb, S., and Henrys, S. (2020). Temporal and spatial variations in seismic anisotropy and VP/VS ratios in a region of slow slip. Earth and Planetary Science Letters, 532:115970.

Zietlow, D. W. (2016). Four Brothers and a Waka: Investigating Lithospheric Accommodation of Shear and Convergence Underlying the South Island of New Zealand. PhD thesis, University of Colorado at Boulder.

Zinke, J. C. and Zoback, M. D. (2000). Structure-related and stress-induced shearwave velocity anisotropy: Observations from microearthquakes near the Calaveras Fault in central California. Bulletin of the Seismological Society of America, 90(5):1305-1312. 